\title{
DISCLAIMER
}

This report was prepared as an account of work sponsored by an agency of the United States Government. Neither the United States Government nor any agency thereof, nor any of their employees, makes any warranty, express or implied, or assumes any legal liability or responsibility for the accuracy, completeness, or usefulness of any information, apparatus. product, or process disclosed, or represents that its use would not infringe privately owned rights. Reference herein to any specific commercial product, process, or service by trade name, trademark, manufacturer, or otherwise does not necessarily constitute or imply its endorsement, recommendation, or favoring by the United States Government or any agency thereof. The views and opinions of authors expressed herein do not necessarily state or reflect those of the United States Government or any agency thereof.

This report has been reproduced directly from the best available copy.

Available to DOE and DOE contractors from the Office of Scientific and Technical Information, P.O. Box 62, Oak Ridge, TN 37831; prices available from (615)576-8401, FTS 626-8401.

A vailable to the public from the National Technical Information Service, U. S. Department of Commerce, 5285 Port Royal Rd., Springfield, VA 22161. 


\title{
FINAL TECHNICAL REPORT
}

\author{
ON
}

\section{CONFIGURATIONAL DIFFUSION OF COAL MACROMOLECULES}

Grant No. DEFG2286PC90504

September 15, 1986 to Sentember 14, 1991

By:

Principal Investigator: James A. Guin Co-Principal Investigator: Christine W. Curtis

Co-Principal Investigator: A. Ray Tarrer Graduate Research Assistants: Seongjae Kim

D. Hwang

C. C. Chen

Z. Chiou

Chemical Engineering Department Auburn University

Auburn, AL 36849 


\section{Configurational Diffusion of Coal Macromolecules}

\section{Executive Summary}

Based on work recently performed in this and other laboratories, it has been found that chemical reactions in catalytic coal liquefaction are greatly influenced by diffusional phenomena, i.e., diffusional resistance involved in transport of molecules in and out of the catalyst support pore structure. These diffusional limitations are detrimental from the standpoint of achieving maximum conversion and selectivity in coal conversion reactions. The nature and extent of this diffusional resistance depends on the diffusion coefficient of the diffusing molecule as it negotiates the tortuous catalyst pore structure, a coefficient which depends in a complex and essentially unknown way on the relative geometries of the diffusing molecule and the catalyst pore structure. It is desirable to obtain fundamental information on the diffusional mechanisms, in the absence of complex ramifications caused by a chemically reacting system. This was the approach taken in our study.

The objective of our research was to obtain fundamental information regarding the functional dependence of the diffusion coefficient of coal molecules on the ratio of molecule to pore diameter. That is, the objective of our study was to examine the effect of molecule size and configuration on hindered diffusion of coal macromolecules through a porous merium. To best accomplish this task, we circumvented the complexities of an actual porous catalyst by using a well defined porous matrix with uniform capillaric pores, i.e., a track-etched membrane. In this way, useful informat:ion was obtained regarding the relationship of molecular size and configuration on the diffusion rate of coal derived macromolecules through a pore structure with known geometry. Similar studies were performed using a pellet formed of porous alumina, to provide a link between the idealized membranes and the actua! complex pore structure of real 
catalyst extrudates. The fundamental information from our study will be useful toward the tailoring of catalysts to minimize diffusional influences and thereby increase coal conversion and selectivity for desirable products.

In our experimental work, the bulk diffusivities and the effective diffusivities of coal macromolecules were measured using the diaphragm cell technique with track-etched polyester membranes, and the relationship between effective diffusivities of coal macromolecules and pore size of a membrane was investigated, in order to obtain fundamental information concerning the configurational diffusion phenomena occurring in coal liquefaction catalysts.

The coal macromolecules were prepared from a sample collected from the deashed residue stream of the ROSE-SR ${ }^{S M}$ unit at the Wilsonville coal liquefaction pilot plant (Wilsonville, Alabama), which was running Illinois No. 6 coal. The track-etched polyester membranes were prepared from irradiated polyester film using optimum etching conditions found our current study. During the experiments, gel permeation chromatography was used to characterize the coal macromolecules and to analyze the diffusion experimental results. The experimental data were analyzed for six coal macromolecule fractions of narrow molecular size range, which were defined in a chromatogram.

Slightly stronger hinderance effects compared to the theoretical curve were observed when the ratios of effective to bulk diffusivities of coal macromolecules were plotted against the ratios of average Stokes-Einstein radii to pore radii. Weak repulsive interactions between coal molecules adsorbed to pore walls and coal molecules diffusing through the pore were postulated to account for differences between the experimental data and the theoretical curve.

Several diffusion studies of model compounds and a diffusional boundary layer study were conducted prior to the coal diffusion study to test the performance of the experimental 
system and to provide information as to the effect of molecular size and the configuration on the effective diffusivity. It was found that the measured diffusion coefficients of model cumpounds were in agreement with the values estimated from the literature, that the measured effective diffusivities of a model macromolecule agreed with those predicted by a hydrodynamic theory with a minor modification, and that the diffusional boundary layer resistance was not affected by membrane properties.

Effective diffusivities of a model macromolecule, tetraphenyl-porphine was measured in actual alumina pellets at two different temperatures. There was a definite relationship between the effective diffusivity and the pore geometry of the alumina nellets, indicating the presence of a configurational diffusion effect. The effect of a ca. $25^{\circ} \mathrm{C}$ change in temperature on the diffusion coefficient was found to be small. 


\title{
Acknowledgements
}

The work reported herein was made possible by the efforts of the following personnel of the Auburn Coal Conversion Laboratory. Their contributions to this report and to the progress of this work are hereby gratefully acknowledged.

\author{
Seongjae Kim \\ Frank Bowers \\ D. Hwang \\ c. Chen \\ z. Chiou
}




\section{Objective and Task Outline}

\section{Objective}

To investigate the phenomenon of hindered diffusion of coal macromolecules in idealized porous media.

\section{Task Outline}

Task 1. Construct a diffusion cell with ideal pore structure for determination of diffusion coefficients.

Task 2. Prepare and characterize ideal porous membranes.

Task 3. Perform model compound experiments to calibrate and test diffusion apparatus and methodology.

Task 4. Prepare and characterize coal macromolecules.

Task 5. Analyze data to evaluate the diffusional behavior of coal macromolecules. 
TABLE OF CONTENTS

LIST OF TABLES $\ldots \ldots \ldots \ldots \ldots \ldots \ldots \ldots \ldots \ldots \ldots \ldots \ldots \ldots \ldots \ldots$

LIST OF FIGURES $\ldots \ldots \ldots \ldots \ldots \ldots \ldots \ldots \ldots \ldots \ldots \ldots \ldots \ldots \ldots$

I. INTRODUCTION $\ldots \ldots \ldots \ldots \ldots \ldots \ldots \ldots \ldots \ldots \ldots \ldots \ldots$

Rationale for This study

Background

II. REVIEW OF LITERATURE ................. 11

Measurement of Liquid Diffusion Coefficient with

a Stokes' Type Diaphragm-Diffusion Cell

Diffusional Boundary Layer Resistance

Transport of Large Molecules in Liquid

Filied Pores

Theory of Hindered Diffusion of Large

Molecules

Experimental studies in Hindered

Diffusion

Track-etched Membranes

Coal Asphaltene

III. PREPARATION AND CHARACTERIZATION OF MEMBRANES

USED FOR HINDERED DIFFUSION STUDY ......... 45

Preparation of Membranes for Hindered

Diffusion Study

Polycarbonate Membranes

Polyester Membranes

Alumina Membranes

Membrane Characterization

Pore Density

Pore Length

Average Pore size

IV. APPARATUS AND PROCEDURES FOR DIFFUSION

EXPERIMENTS ........................ 83

Apparatus for Diffusion Experiments

Experimental Procedures 
V. DIFFUSIONAL BOUNDARY LAYER STUDIES .........

Experiments

Sucrose and water system

$\mathrm{N}$-octane and $\mathrm{N}$-decane system

Results and Discussion

Evaluation of Boundary Layer Resistances

for Membranes Having Small Pore Sizes

Diffusion Experiments with Alumina Membranes

Experiments

Results and Discussion

VI. DIFFUSION OF MODEL COMPOUNDS ............. 120

Diffusion Experiments with Paraffinic

Hydrocarbons

Experiments

Results and Discussion

Selection of Solvent and Diffusivity

Measurements for $\mathrm{N}$-octane in Ethyl Acetate

Experiments

Results and Discussion

Diffusion Experiments with Polycyclic

Aromatic Hydrocarbons

Experiments

Results and Discussion

Diffusion Experiments with a Model

Macromolecule

Experiments

Results and Discussion

VII. DIFFUSION OF COAL MACROMOLECULES .......... 171

GPC Calibration

Experiments

Results and Discussion

Effective Diffusion Coefficient

Measurement of Coal Macromolecules

Experiments

Results and Discussion

VIII. SUMMARY AND CONCLUSIONS ................ 203

IX. RECOMMENDED AREAS OF FUTURE WORK ........... 209

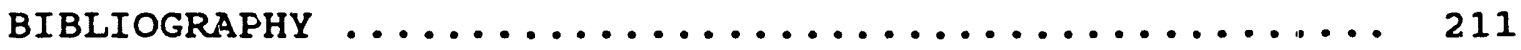

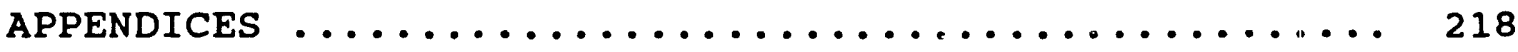

A. Derivation of the Equation for a Dorous Membrane Transport 
B. Sample Calculations for the Analysis of the Experimental Data

c. A solute Flux through Polycarbonate Membrane Matrix

D. Estimation of the Diffusion Coefficient of $\mathrm{N}$-octane in $\mathrm{N}$-decane

E. Theoretical Curves for the Hindrance Factor

F. Error Analyses

G. BASIC Programs for Data Acquisition and Handling

H. Diffusion in Actual Alumina Porous Media

I. Nomenclature 
LIST OF TABLES

2.1 Comparison Table for Sherwood Number

Correlations ......................... 19

2.2 Comparisons of Properties between Petroleum-and Coal-Derived Asphaltenes ................ 43

3.1 Average Pore Diameters (Prepared Alumina

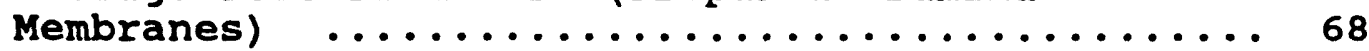

3.2 Actual Pore Diameters from Measurements (Nuclepore $^{\mathrm{TM}}$ Polycarbonate Membranes)........... 74

3.3 Average Pore Diameters Determined from Diffusion Experiments (Prepared Polyester Membranes) .... 82

5.1 GC Conditions for Analysis $\ldots \ldots \ldots \ldots \ldots \ldots \ldots$

5.2 Results of Diffusion Experiments with $0.01 \mu \mathrm{m}$ Membranes (N-octane $/ \mathrm{N}$-decane) @ $25^{\circ} \mathrm{C} \ldots \ldots \ldots 105$

5.3 Properties of Alumina Membrane ............ 110

5.4 Results of the Permeability Tests of Allumina Membranes .................. 111

5.5 Results of Diffusion Experiments with Alumina Membranes (Sucrose (solute)/Water(solvent)) e $25^{\circ} \mathrm{C}$

5.6 Results of Diffusion Experiments with Alumina Membranes (N-octane (solute) / N-decane (solvent)) e $25{ }^{\circ} \mathrm{C} \ldots \ldots \ldots \ldots \ldots \ldots \ldots \ldots \ldots \ldots \ldots \ldots . \ldots \ldots 114$

6.1 GC Column Temperature Condition for Each

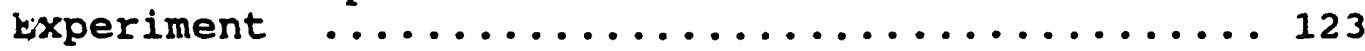

6.2 Summary of Measured Diffusion Coefficients for $\mathrm{N}$-paraffinic Hydrocarbons in $\mathrm{N}$-decane

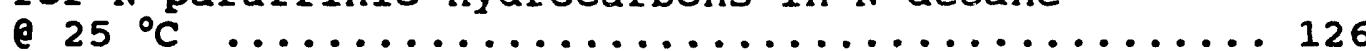

6.3 Summary of Diffusion Coefficients from the Direct and Indirect Measurements ............... 128 
6.4 Results of Indirect Diffusion Coefficient Measurements $\left(\mathrm{C}_{8} / \mathrm{N}\right.$-decane and Ethy? Acetate)

e $25^{\circ} \mathrm{C}$ with Alumina Membranes ............. 134

6.5 Results of Indirect Diffusion Coefficient

Measurements ( $\mathrm{C}_{8} / \mathrm{N}$-decane and Ethyl Acetate)

a $25{ }^{\circ} \mathrm{C}$ with Polyester Membrane ............. 135

6.6 Diffusion Coefficients for $\mathrm{N}$-octane in

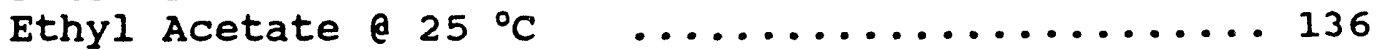

6.7 Diffusion Coefficients of N-uctane in Ethyl

Acetate from the Different Bounaary Layer

Empirical Equations $\ldots \ldots \ldots \ldots \ldots \ldots \ldots \ldots \ldots \ldots$

5.8 UV Operating Conditions for Model Compounds ... 141

6.9 Diffusion Coefficients of 10 Polycyclic Aromatic

Hydrocarbons in Ethyl Acetate e $25^{\circ} \mathrm{C} \ldots \ldots \ldots 142$

6.10 Summary of Diffusion Coefficients Obtained

from the in situ-Measurements and the

Separated Measurements ................. 144

6.11 Molecular Dimensions of 10 Polycyclic Aromatic

Hydrocarbons Determined from Modeling Work ..... 148

6.12 Measurements of the Bulk Diffusion Coefficient

of TPP in Ethyl Acetate e $25^{\circ} \mathrm{C} \ldots \ldots \ldots \ldots \ldots \ldots . . \ldots 154$

6.13 The Results of the Hindered Diffusion Experiments

of TPP in Ethyl Acetate $225{ }^{\circ} \mathrm{C} \ldots \ldots \ldots \ldots \ldots . \ldots 155$

6.14 The Results of statistical Tests for the

Hindered Diffusion Data of $\mathrm{mPP} \quad \ldots \ldots \ldots \ldots . \ldots 159$

6.15 Changes in Calculated Pore Diameter of Polyester

Membranes During the Hindered Diffusion

Experiments of TPP

7.1 Molecular Weights of Polystyrenes and GPC

Elution Volumes

7.2 Polycyclic Aromatic Hydrocarbons and Porphine used as GPC Standards and GPC Operating

conditions

7.3 Changes in Calulated Pore Diameter of Membranes Used in Hindered Diffusion of Coal.

Macromolecules 
7.4 Bulk Diffusion Coefficients of coal

Asphaltene Fractions

7.5 Iffective Diffusion Coefficients of Coal Asphaltene Fractions

7.6 The Resultis of Statistical Tests for Hindered Diffusion Data of Coal Macromolecules ........ 197

D.1 Sources of Diffusivity Data e $25{ }^{\circ} \mathrm{C}$ Used in the Calculation $\ldots \ldots \ldots \ldots \ldots \ldots \ldots \ldots \ldots \ldots \ldots$

D.2 Comparison of the Methods for the Estimation of Some Paraffinic Hydrocarbons' Diffusivities .... 247

F.1 Effect of Errors in Pore Length on Uncertainties of Pore Sizes Determined from the Various

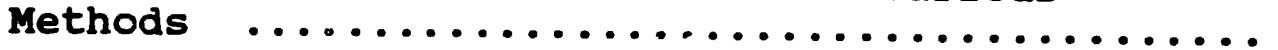

F.2 Relative Errors in Diffusivity Data of TPP and Coal Macromolecules Affected by the Errors

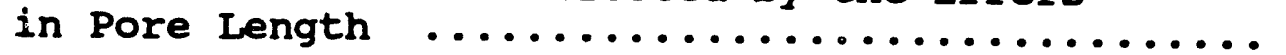

F.3 Effect of Errors in Pore Density on Uncertainties of Pore Sizes Determined from the Various

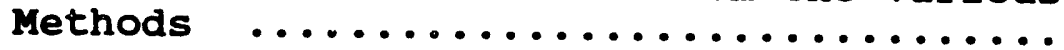

F.4 Relative Errors in Diffusivity Data of TPP and Coal Macromolecules Affected by the Errors in Pore Density ....................... 257

H.1 Diffusivity Values in TPP-Ethyl Acetate system .. 271 


\section{LIST OF FIGURES}

1.1 Effect of Diffusional Path Length on Conversion of Coal to Pentane Soluble oils

2.1 Hard sphere in Cylindrial Pore $\ldots \ldots \ldots \ldots \ldots \ldots 22$

2.2 Hypothetical cross-sectional View of coal Asphaltene Proposed by Yen ................44 44

3.1 Etching Curve of Polycarbonate Films (2 $\mathrm{N} \mathrm{NaOH}$ Sol'n, 0.01 wto Dowfax ${ }^{\mathrm{T}}$ \& $\left.20^{\circ} \mathrm{C}\right) \ldots . . . \ldots . . . .49$

3.2 Effect of Caustic Concentration on the Etching Rate ............................ 51

3.3 Effect of Temperature on the Etching Rate .... 52

3.4 Arrhenius plot of Etching Rates ........... 53

3.5 Etching Curve of Polyester Films (1N NaOH sol'n, 0.02 wtg Dowfax $\left.{ }^{\text {TM }} 80^{\circ} \mathrm{C}\right) \ldots \ldots \ldots \ldots \ldots . \ldots \ldots$

3.6 Model for the structure of Porous Aluminum Oxide

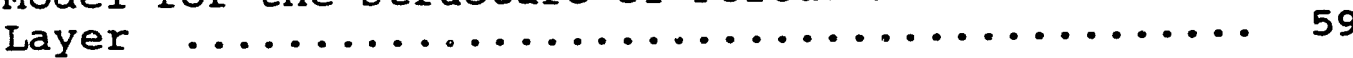

3.7 Diagram for the Anodization Cell $\ldots \ldots \ldots \ldots \ldots \ldots$

3.8 Apparatus for the Liquid Permeability Experiments in Anodic oxidation Method ....... 66

3.9 Scanning Electron Microscope Picture of Folycarbonate Membrane $(5 \mu \mathrm{m}) \quad \ldots . . . . . . . .470$

3.10 Scanning Electron Microscope Picture of Polycarbonate Membrane $(0.2 \mathrm{~mm}) \quad \ldots . . . . . . .70$

3.11 Scanning Electron Microscope Picture of Polyester Membrane $(1 \mu \mathrm{m}) \quad \ldots . . \ldots \ldots . . . \ldots .71$

3.12 Scanning Electron Microscope Picture of Polyester Membrane $(1 \mu \mathrm{m}) \quad \ldots . \ldots \ldots \ldots . . \ldots . . .71$

3.13 Liquid Permeability Test Apparatus ......... 75 
3.14 Gas Permeability Test Apparatus ........... i8

4.l Schematic Drawing of Half Diffusion Cell ..... 84

4.2 Schematic Drawing of stirrer $\ldots \ldots \ldots \ldots \ldots \ldots$

4.3 Schematic Drawing of Diffusion Experimental

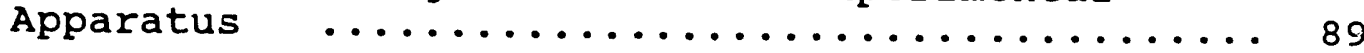

$5.1 \mathrm{~N}_{\mathrm{Sh}}$ vs $\mathrm{N}_{\mathrm{Re}}$ Correlation for $0.1 \mu \mathrm{m}$ Membrane Data

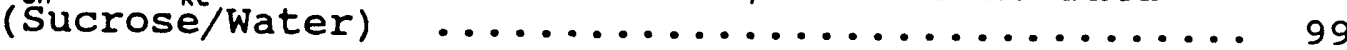

$5.2 \mathrm{~N}_{\mathrm{Sh}}$ vs $\mathrm{N}_{\mathrm{Re}}$ Correlation for $0.1 \mu \mathrm{m}$ Membrane Data

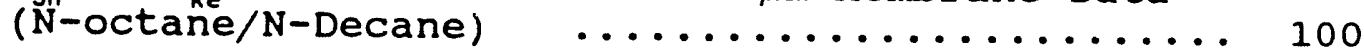

$5.3 \mathrm{~N}_{\mathrm{Sh}}$ vs $\mathrm{N}_{\mathrm{Re}}$ Correlation for $0.05 \mu \mathrm{m}$ Membrane Data

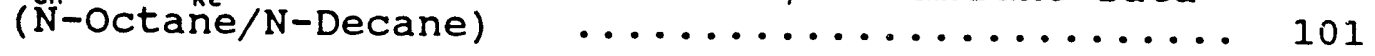

$5.4 \mathrm{~N}_{\mathrm{Sh}}$ vs $\mathrm{N}_{\mathrm{Re}}$ Correlation for All Diffusion

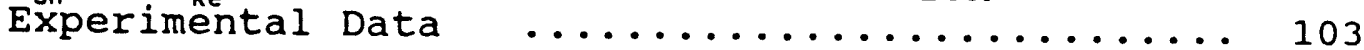

5.5 Boundary Layer Resistances from Experiments

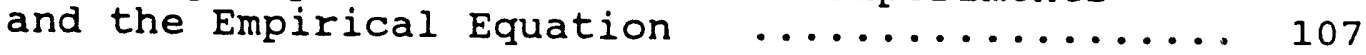

5.6 Estimation of the Tortuosity Factor of Alumina

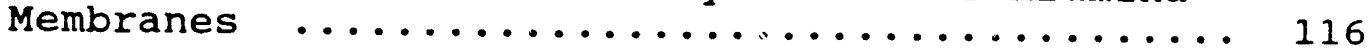

5.7 $\mathrm{N}_{\text {Sh }}$ vs $\mathrm{N}_{\mathrm{Re}}$ Correlation for Diffusion Data with Alumina Membranes ............................... 117

6.1 Diffusion Coefficients vs. Number of Carbon Atoms in Polycyclic Aromatic Hydrocarbons .... 145

6.2 Diffusion Coefficients vs. Average Planar Molecular Radii of Polycyclic Aromatic

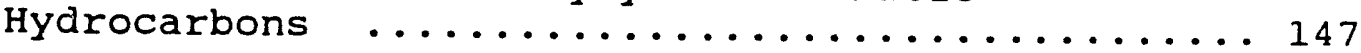

6.3 Chemical Structure of Tetraphenylporphine .... 150

6.4 UV Calibration Curve for a TPP Solution

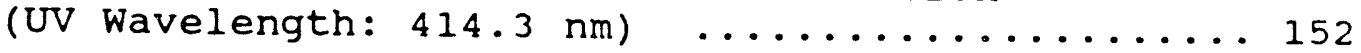

6.5 Dependence of $\mathrm{D}_{\mathrm{e}} / \mathrm{D}_{\infty}$ on $\lambda$ in Hindered Diffusion

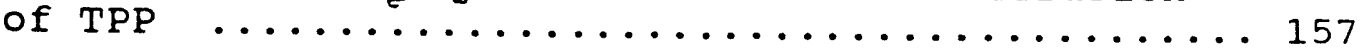

6.6 Changes in Theoretical Curve and the Hindered

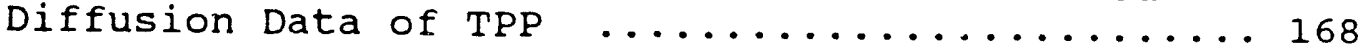

7.1 GPC Calibration Curve for Coal Asphaltene .... 178

7.2 GPC Elution Curve of Coal Asphaltene Solution .. 180 
7.3 Area of Each Coal Fraction in Chromatogram vs.

Concentration of standard solution .......... 188

7.4 Relative Area vs. Relative Concentration ...... 189

7.5 Dependence of $\mathrm{D}_{\mathrm{e}} / \mathrm{D}_{0}$ on $\lambda$ in Hindered

Diffusion of Coal Asphaltene $\ldots \ldots \ldots \ldots . \ldots 196$

A.1 Drawing for Track-Etched Membrane Transport .... 220

B.1 GPC Chromatogram of the stock Solution ....... 225

B. 2 GPC Chromatogram of the Samples from the

Top and the Bottom compartment of the

Diffusion Cell

C.1 Etching Curves of Polycarbonate Films Determined from $c_{8} /$ Ethyl Acetate system ...... 238

C.2 FTIR Spectrum of a Polycarbonate Film ....... 240

C. 3 FTIR Spectrum of a Polycarbonate Film Treated

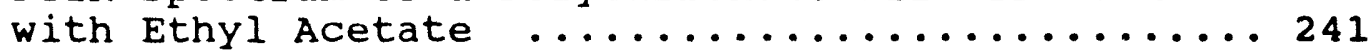

E.1 Theoretical Curves for the Hindrance Factor .... 249

F.1 Sensitivity Analysis of TPP Diffusion Data .... 258

F.2 Sensitivity Analysis of Coal Diffusion Data ... 259

H.l Diagram of glass diffusion cell ........... 272

H.2 Beer's Law plot for analysis of TPP in ethyl

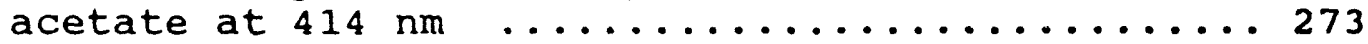

H.3 Typical diffusivity data for TPP Ethyl Acetate

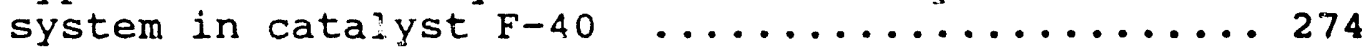

H.4 Diffusional adsorption uptake curves for $\mathrm{TPP} / \mathrm{mineral}$ oil solution on alumina pellets ... 275

H.5 GPC chromatograms of diffusional uptake curves of coal macromolecules from THF solutions at

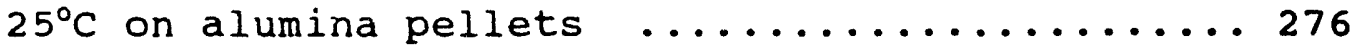

H.6 GPC chromatograms of diffusional uptake curves of coal macromolecules from THF solutions at $25^{\circ} \mathrm{C}$ and $50^{\circ} \mathrm{C}$ on alumina pellets 
I. INTRODUCTION

\section{Rationale for This study}

Chemical reactions occurring in heterogeneous catalysts involve a series of sequential events; i.e., reactants diffuse into the porous structure, reartion takes place at active sites, and the formed products diffuse back to the bulk fluid. Thus, the overall reactions are influenced by diffusional phenomena involved in transport of molecules in and out of the catalyst support pore structure.

The influence of diffusional limitation on overall reaction becomes more significart in the catalytic process, where large molecules are involved in the reaction as seen in heavy-end upgrading processes or direct coal liquefaction processes. In these types of processes, the diffusional transport rates of reactants within the pores of the catalyst support are drastically reduced from those within the free solution. This phenomenon of retarded diffusion of large molecules in the liquid-filled pores of molecular dimension, as observed in these processes, is called hindered, restricted, or configurational diffusion. Figure 1.1 depicts results from a hindered diffusional effect study in catalytic coal liquefaction recently conducted by Rhee (1). According to this study, the 
powdered form catalyst (representing shorter diffusional path length) yielded significantly greater oil production than the extrudate pellets. This data is a clear indication of the influences of pore diffusional restriction in reducing the oil yield with extrudate pellets.

Evidence of hindered diffusional influence in catalytic coal liquefaction can be manifested in several ways, including catalyst activity, catalyst selectivity and catalyst deactivation.

Catalyst activity. The presence of a diffusional limitation will generally retard the rate of the overall conversion process, since the reacting coal moieties reach the catalytic active sites through diffusional paths of finite length. In the process, the mass transfer and kinetic processes generally occur in series.

Catalyst selectivity. Since the diffusion rate $=$ of particular species, e.g. asphaltenes, preasphaltenes, etc., will generally depend on the molecular size as well as the pore size of the support, different species will diffuse to the catalytic surface at different rates, leading to a selection process which will influence the overall product distribution. In addition, the selectivity for the formation of a hydroaromatic species, generally an intermediate product, is reduced by pore diffusional resistances, since such resistances tend to favor the production of the terminal product, i.e., the fully 


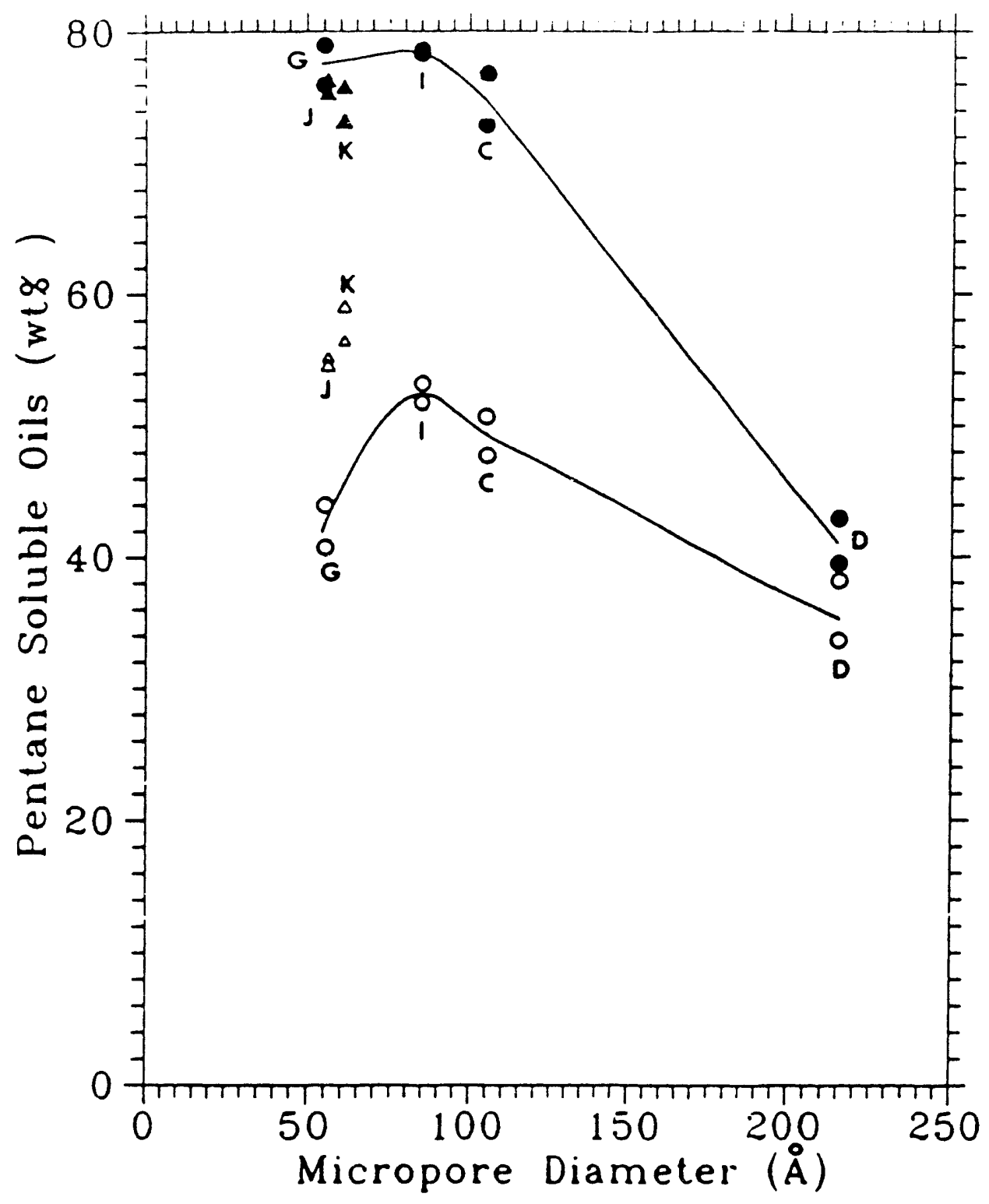

Figure 1.1 Effect of Diffusional Path Length on Conversion of Coal to Pentane Soluble Oils (1). 
saturäted species, in a series-reaction pathway. Thus, the presence of intraparticle diffusion resistance is detrimental to the maintenance of a coal solvent with high hydroaromatic content.

Deactivation. One of several mechanisms which cause the deactivation of supported extrudate catalysts is the reduction in pore diameter which eventually leads to pore plugging. This is caused by deposition of carbonaceous and metallic deposits during the reaction process. These factors serve to alter the pore geometry in a manner that is detrimental to the diffusion rate of the reacting species; hence, the overall diffusion-reaction process is retarded. Experimental studies concerning the evidence of diffus onal limitations in coal systems were conducted by several groups in the literature. Many groups (2-8) investigated the effects of catalyst pore size or catalyst pellet size on the conversion of coal or bituminous materlals, and generally reported that the conversion of reactants per unit catalyst surface area increased as pore diameters increased or as catalyst pellet sizes decreased. Some studies pointed out the existence of an optimum pore diameter of catalyst because of the reduction in surface area per unit reactor volume for the larger pore diameter catalyst $(1,6)$. Other groups observed the effect of diffusional limitation on the product distribution in catalytic coal 1 iquefaction (10-12). The slower 
deactivation rate of the larger pore diameter catalyst was reported by stiegel (j)."

- The effect of pore diffusional limitations on the overall rate of a catalytic reaction is viewed in terms of the effectiveness factor, defined as the ratio of a component's reaction rate in the presence and absence of pore diffusion resistances. An understanding of the role of pore diffusion as it affects different stages of coal liquefaction is essential to make the best use of a catalyst and to provide an optimum reactor design. such an improved understanding is a major motivation of the current study. Additional research is needed into the fundamental nature of diffusional mechanisms operating in this situation so that improvements in the performance of the extrudate-type catalyst can be effected. For example, through alteration of the pore size distribution of the extrudate support, it may be possible to approach the result obtained with the powdered form of the catalyst.

Coal asphaltenes, which are defined by their solubility class, are widely accepted as a key intermediate in direct coal liquefaction mechanisms (13). However, no previous studies have reported on either diffusion coefficient measurements of coal asphaltenes or degree of hindrances of coal asphaltenes confined in the pores, both of which are key elements in the study of the diffusional effect in catalytic coal liquefaction. 
In general, diffusion studies conducted with catalyst support materials entail considerable vagueness due to the complex structure of catalyst support as well as possible interactions with solutes and pore walls of the catalyst support. In the present study, track-etched membranes having ideal cylindrical pores were adopted to minimize this lack of explicitness. Thus, a fundamental diffusion study of coal asphaltene using this type of membrane will provide valuable information for understanding the complex nature of diffusional phenomena occurring in catalytic coal liquefaction.

\section{Background}

Three major diffusion mechanisms contribute to the transport of molecules in the porous network of a catalyst support. An understanding of these mechanisms is important for an appreciation of the current research. Thus, brief reviews of these mechanisms are provided as follows.

Bulk diffusion in a porous medium. The flux of a solute in macropores within porous matrix is reduced from its value in the bulk solution by simple geometric factors and can be expressed as:

$$
N_{A}=-\frac{\epsilon D_{\infty} \nabla C}{\tau}
$$

where $D_{0}$ is the ordinary molecular diffusivity of solute in the solution, $\epsilon$ is the porosity and $\tau$ is the tortuosity of the porous medium. In Equation $1.1, N_{A}$ is the flux of $A$ in 
7

moles/s. $\mathrm{cm}^{2}$ of porous matrix. The porosity, $\epsilon$, reduces the flux simply because the fraction, $1-\epsilon$, of the total area is occupied by the solid and is thus not available for diffusion. The porosity is a well-defined property of the porous medium and can be measured by displacement techniques. The tortuosity is an attempt to account for several factors, including the fact that the actual diffusion path length is lengthened through the porous matrix by the (usually) circuitous route of the pores as well as the fact that the pores contain constrictions and are of nonuniform diameter. Both of these phenomena serve to reduce the effective diffusivity, $D_{e}$, which may be defined in Equation 1.2 as:

$$
D_{e}=\frac{\epsilon D_{-}}{\tau}
$$

Restricted diffusion. When molecules of comparable size with the pores diffuse through the porous matrix, the molecules experience an increased "frictional" drag (hydrodynamic resistance) due to the confinement of the fluid by the pore walls, over and above the drag that which would be experienced if the molecules were diffusing in an unconfined solvent. Also, a concentration of molecules in the pore is different from that of bulk solution. The ratio of pore to bulk concentration is called the partition coefficient which is another major factor causing the reduction of large molecule's diffusivity in the confined 
pores. This partition coefficient is subject to

interactions between pore walls and diffusing molecules. A simple geometric consideration of these interactions leads to the steric partitioning.

Surface diffusion. When diffusing species are adsorbed onto the solid phase in a porous matrix, the adsorbed phase is not necessarily stationary, and diffusion of adsorbed molecules within this phase can contribute significantly to the total flux within the porous medium. Such a flux of adsorbed molecules is referred to as surface diffusion (14). In some cases, the contribution to the total diffusion flux from surface diffusion can be substantial, exceeding the contribution from pore volume diffusion. If the solute is not adsorbed onto the solid phase, the contribution of surface diffusion vanishes. The surface diffusion contribution is usually modeled as an additional flux with a Fick's law-type expression with a surface diffusion coefficient, $D_{s}(15)$.

Many diffusion studies have been carried out to obtain a better understandirg of each diffusion mechanism. In general, a relatively small number of experimental studies on surface diffusion have been conducted. This is because many researchers working on catalysts have considered the contribution of surface diffusion to the overall transport of molecule insignificant due to its relatively slower rate (about two orders of magnitude smaller than the 
corresponding bulk diffusivities (16)). Also setting up an experimental scheme to observe surface diffusion as an isolated phenomenon occurring in the pores has proven somewhat difficult. However, in the pore diffusion of molecules with high adsorptivity, this type of diffusion can be a substantial contribution to the overall molecular transport. Thus, in the experimental work conducted in the present research, the surface diffusional flux was minimized by the utilization of porous media having low adsorptivity and low internal surface area, i.e., polymer track-etched membranes. The main concerns in the present research center on the restricted diffusion of coal macromolecules in micr.. - es having molecular dimensions.

The phenomenon of hindered diffusion is important not only in the area of catalysts but also in other application areas such as ultrafiltration, reverse osmosis, size exclusion chromatography, membrane dialysis, and enhanced oil recovery, where the transport of solutes occurs through pores having comparable dimensions with solutes. Thus, many theoretical and experimental studies on hindered (restricted or configurational) diffusion have been conducted by researchers working in different areas. Details of these previous works will be reviewed in Chapter II.

However, it should be noted that no work has been corducted on hindered diffusion of coal macromolecules. Hence, the measurements of bulk and effective diffusion 
10

coefficients of coal macromolecules and the evaluation of experimental data in the light of currently available hindered diffusion theories will be primary focal points in the present research. In the current research, the experimental measurements of coal molecules' bulk and effective diffusion coefficients were made using the stokes' diaphragm-diffusion cell technique. 


\section{REVIEW OF LITERATURE}

\section{Measurement of Liquid Diffusion Coefficient with a Stokes' Type Diaphragm-Diffusion Cell.}

The diaphragm diffusion cell was first developed by Northrop and Anson in 1928 (17). Since then, it has been improved experimentally and theoretically by many researchers $(18-20)$. In 1950, stokes (21) introduced the Stokes' type diffusion cell, which provided the basis by which the diaphragm cell terhnique was recognized as a reliable method for measuring diffusion coefficients in the liquid phase.

A diaphragm diffusion cell consists of two compartments separated by a porous membrane. The two compartments are initially filled with solutions of different concentrations, and the transport rate of solute through the membrane from one compartment to the other is determined from the concentration changes of solute in two compartments before and after the experiment. During the experiment, two compartments of the diffusion cell are stirred by magnetic stirrers to maintain a homogeneous concentration within each compartment and to minimize the diffusional boundary layer resistance. The diaphragm cell technique is directly applicable to the present study because it allows one to 
observe the phenomenon of hindered diffusion of large molecules in idealized porous media.

In the technique, the diffusion coefficient of solute is evaluated by the use of an analytical equation obtained from the material balance describing the concentration changes of solute in twe compartments of a diffusion cell. The equation is derived from a so-called pseudo-steady state assumption, and is expressed as:

$$
\ln \left(\frac{C_{H}-C_{L}}{\dot{C}_{H}-\dot{C}_{L}}\right)=-\frac{A \cdot t}{R_{T}} \cdot\left(\frac{1}{V_{H}}+\frac{1}{V_{L}}\right)
$$

In the pseudo-steady state assumption, solute flux throughout the membrane at any given time is assumed to be constant. Meanwhile, the concentrations of solute in the two compartments of the diffusion cell change with time. The validity of this assumption has been discussed in the literature $(22-24)$.

In the present study, mainly track-etched membranes were used as barriers in the diffusion cell. These membranes had almost negligible total pore volumes compared to the compartment volume of the diffusion cell. Also, the pore length to diameter ratio was at least greater than 300 . In addition, the concentrations of solutes used in the current study were very dilute, so there were no volume changes of mixing before or after the diffusion experiment. All of these experimental conditions support the pseudosteady state assumption. 
Malone and Anderson (25) studied the hindered diffusion of latex particles through track-etched membranes, and also derived the following equation relating the total transfer resistance $\left(R_{T}\right)$ obtained from Equation 2.1 to the diffusion coefficient of solute.

$$
R_{T}=\frac{I}{D_{\theta} n \pi I_{0}^{2}}+\frac{\pi r_{0}^{2} / 2}{D_{\infty} n \pi I_{0}^{2}}+\frac{2 \delta}{D_{\infty}}
$$

In this equation, the first term of the right side depicts the membrane resistance, the second term represents the entrance and exit effects, and the third term describes the boundary layer resistance on each side of the membrane. Details of assumptions and steps in deriving Equation 2.1 are provided in Appendix A. The validity of this expression has been proven by several groups who conducted hindered diffusion studies with track-etched membranes (26-28).

The measurement of effective diffusivities of coal macromolecules as shown in the first term of Equation 2.2 is the focal point of current research. Since the value of total transfer resistance $\left(R_{T}\right)$ is the only measurable quantity from diffusion experiments, the effective diffusivities of coal macromolecules must be evaluated by use of an analytical technique based on some experimental and theoretical considerations related to the hindered diffusion experiment. The following sections will review these considerations. 


\section{Diffusional Boundary Layer Resistance}

Correct analysis of intrinsic membrane resistance requires the accurate evaluation of the boundary layer resistance. In several previous hindered diffusion studies with track-etched membranes, contributions of the boundary layer resistances to total transfer resistances were considered insignificant and were subsequently neglected $(29,30)$. However, a few studies concerning the diffusional boundary layer reported that the boundary layer resistance increased for low porosity membranes $(27,28)$. These results somewhat conflict with the assumption used in some hindered diffusion studies which neglects the boundary layer resistance. As an attempt to elucidate these conflicts, an experimental study concerning the diffusional boundary layer behaviors in diffusion experiments was concucted in the current research. Details are described in Chapter $V$. Here, previous studies on this subject are briefly reviewed. The boundary layer resistance in membrane transport has been studied by several groups $(27,28,31-33)$. Most of the resulting empirical equations obtained in these studies took the form of a dimensionless group relationship as shown in Equation 2.3:

$$
N_{S h}=\alpha \quad N_{R \theta}^{m} N_{S C}^{q}
$$

Here, the values of $\alpha, m$ were determined from experimental results, and depend on the experimental conditions used in each study. The numerical value of the 
exponent term, $\mathrm{q}$ in the schmidt number was found to be close to 0.33 both theoretically and experimentally (22).

Kaufmann and Leonard (32) measured the interfacial boundary layer resistance for the diffusion of three different sugars through homogeneous cellophane membranes in a diaphragm diffusion cell. The diffusion cell was installed horizontally and equipped with internally mounted rotating impellers. In the analysis of experimental results, the values of membrane resistances used in isolating the boundary layer resistance from the total transfer resistance were obtained by the wilson plot method. In this method, a series of total transfer resistances evaluated at different stirring speeds were plotted on a semi-log scale, and the value of the membrane transfer resistance was obtained from the intercept of the correlation curve. The intercept represented the total transfer resistance including no boundary layer resistances at an infinite stirring rate. The reported values of $\alpha, \mathrm{m}$, q obtained froin multiple regression analysis of 52 experimental points of data were $0.105,0.68$ and 0.32 , respectively.

colton and Smith (33) used a benzoic acid plate dissolved into an aqueous stirred phase, simulating the boundary layer behavior of one side of a homogeneous membrane in a diffusion cell, to study the diffusional boundary layer resistance. An empirical correlation of the 
mass transfer coefficient data, at different stirring speeds, into Equation 2.3 yielded a transition point from a laminar region to a turbulent region at a Reynolds number of about $3 \times 10^{4}$. According to this study, the value of $m$ was 0.567 in the laminar region and 0.746 in the turbulent region.

Levich (34) conducted a theoretical analysis of mass transfer through a laminar boundary layer and found the values of $\alpha$ and $m$ to be 0.62 and 0.5 .

Malone and Anderson (31) studied the diffusional boundary layer resistance in the diaphragm diffusion cell with a heterogeneous membrane, where the flux of solute occurs only through the discrete pores of the membrane. Membranes used in this study were well-characterized tracketched mica membranes.

In the study, the diffusivities of potassium chloride in an aqueous solution at different stirring rates were observed, and experimental results were analyzed by use of the Equation 2.2 to obtain the diffusional boundary layer resistances. A least square fit of their data into the correlating Equation 2.3 determined the value of $\alpha$ and $m$ to be 0.13 and 0.58 , respectively. The study also reported that the experimental boundary layer thicknesses were greater (by a factor of 3) than those predicted by an empirical equation determined for a homogeneous membrane in the literature. 
17

Similar studies were conducted by Bohrer (27) with track-etched polycarbonate membranes having different pore sizes and porosities. The solutes used in this study were urea, glucose, sucrose, ficoll, and dextran. The polarographic technique was used as another experimental technique to measure boundary layer resistances and to compare the results with those obtained from the diffusion cell technique.

The observed values of boundary layer resistance for the lowest porosity membrane $\left(n \pi r_{0}^{2}: 0.017\right)$ were two or three times greater than the corresponding values for the other membranes having a larger porosity $(0.062,0.199)$. Bohrer also reported an agreement between the values of boundary layer resistance for highest porosity membrane (0.199) and the values obtained from the polarographic method which were simulating the boundary layer study with the homogeneous membrane. The value of $\mathrm{m}$ in Equation 2.3 was found to be 0.66 .

Another noteworthy result of this work was the comparison of methods for determining the intrinsic membrane resistance used in boundary layer studies. Bohrer found that the values for intrinsic membrane resistance obtained from the wilson plot method were not in agreement with the corresponding values obtained from their empirical equation. He attributed this difference to difficulties in locating the intercept in the wilson plot method. 
In another study conducted by Bohrer and co-workers (28), the authors reported substantial increases in diffusional boundary layer resistance for low porosity membranes. In this work, dextran and ficoll were used as solutes and track-etched polycarbonate membranes were employed in the diffusion cell.

One of the important aspects found in the literature concerning the diffusional boundary layer behavior was that evidences of increase in boundary layer resistance existed in several experimental studies using low porosity tracketched membranes. Thus, a further experimental investigation on the boundary layer resistance was required in the current study since track-etched membranes used in hindered diffusion experiments tend to have low porosities due to their small pore sizes.

In Table 2.1, a summary of previous studies for Sherwood number correlation is provided. Direct comparison of these empirical equations is not possible due to the differences in experimental conditions used in each study. However, it is observed that the reported values of the exponent term of the Reynolds number in the turbulent region varies from 0.58 to 0.746 in this table.

\section{Transport of Large Molecules in Liquid Filled Pores}

In this section, discussion will center on the membrane resistance, the first term in Equation 2.1. As noted in the 
Table 2.1

Comparison Table for Sherwood Number Correlations

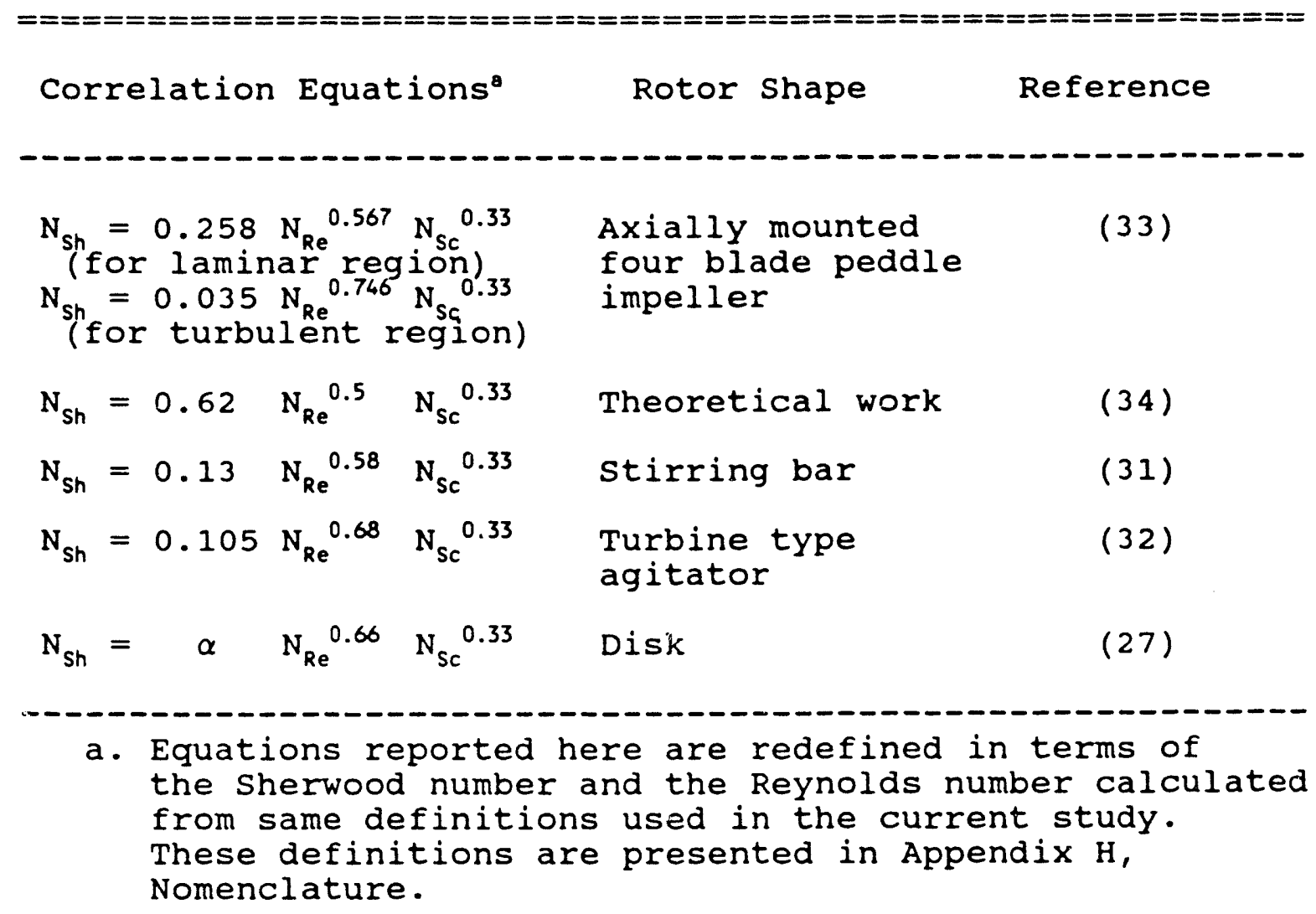


equation, the membrane resistance term includes the effective diffusivity of solute, $D_{e}$, usually expressed as:

$$
D_{\theta}=D_{-} \cdot f(\lambda)
$$

Here, the function $f(\lambda)$ is a term of correction which accounts for the reduction in diffusivity of solute in the pores compared to the diffusivity of solute in the bulk solution.

A study of the movement of large solutes in confined pores has been the subject of many researchers working in the area of membrane science (including biological membranes), chromatography, catalysts and physiology. Early studies on this subject were mostly conducted by biological research groups examining the transport of large biological molecules through membranes. Ferry (35) was probably the first to introduce the concept of a partition coefficient from a steric consideration to derive a quantitative expression for convective transport of large biological molecules through membranes.

An approximate equation for treating the hydrodynamic drag effect used in many hindered diffusion studies with large molecules was derived by Faxen (36) from the theoretical consideration of axial sedimentation of a rigid sphere in a cylindrical tube.

Pappenheimer et al. (37) and Renkin (38) first suggested the combination of these two effects to account for the reduced diffusivity of solute in micropores. They 
21

observed the diffusion rate of organic nonelectrolytes through membranes having very small pores, and proposed the following expression, now known as Renkin's equation:

$$
D_{\theta} / D_{\infty}=(1-\lambda)^{2} \cdot\left(1-2.104 \lambda+2.089 \lambda^{3}-0.948 \lambda^{5}\right)
$$

Here $\lambda$ is the ratio of the molecular to pore diameter. In this equation, the first term on the right-hand side accounts for the geometric exclusion of the diffusing molecule within the pore, while the second term represents an increased hydrodynamic drag.

In 1970, the development of the track-etched membrane stimulated experimental research in this area, becaluse its straight, identical, cylindrical shaped pores provided an ideal membrane for hindered transport study. Since that time, many studies on this subject have been conducted. Most of these works were reviewed extensively by Deen (39). The following theoretical review on hindered diffusion of large molecules was primarily taken from Deen's work. A brief look at previous experimental work relevant to the present study is provided in the next section following the theoretical review.

\section{Thecry of Hindered Diffusion of Large Molecules}

A derivation of the solute flux equation for large molecules in one cylindrical pore requires several assumptions; one is that the solute is a rigid sphere 


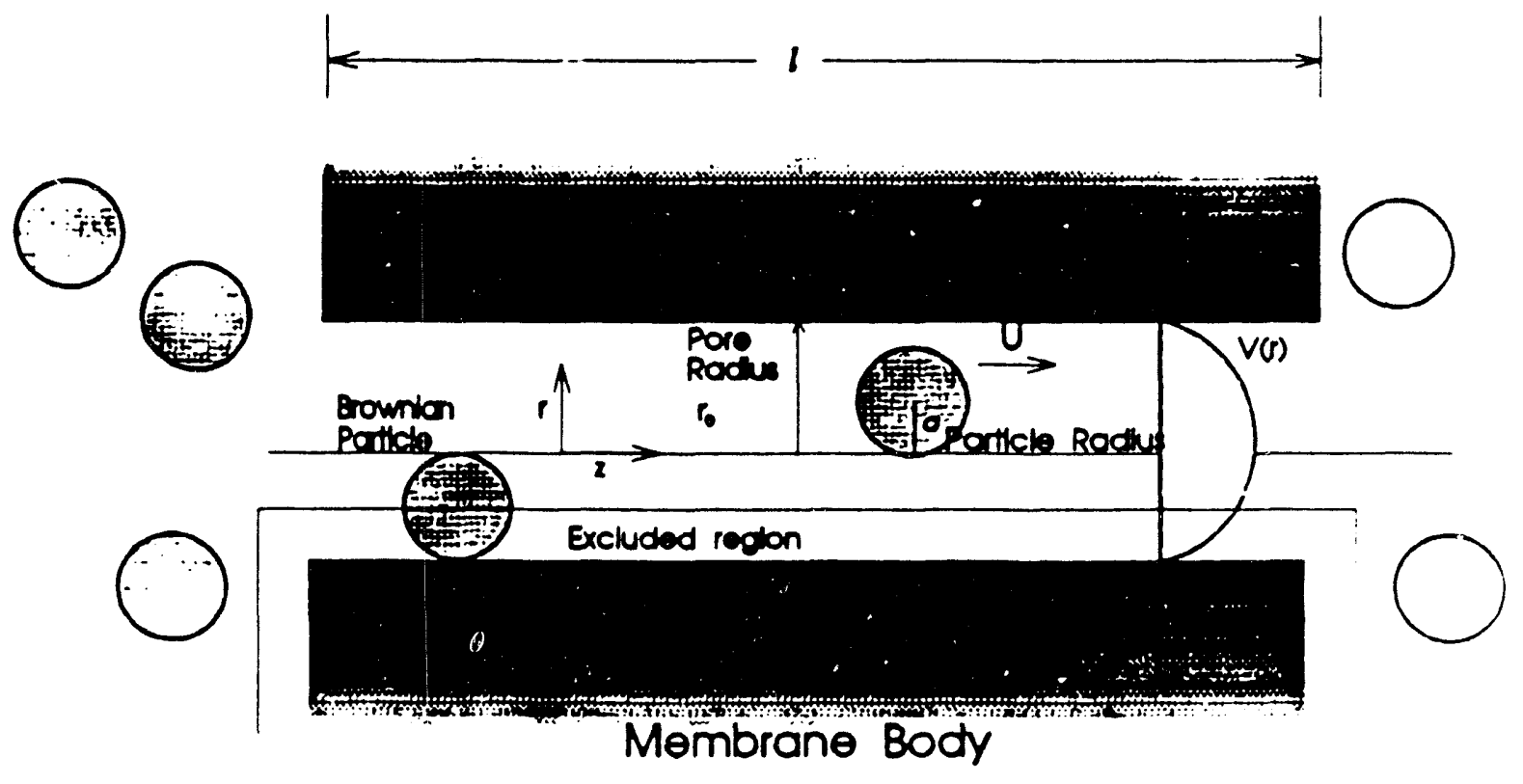

Figure 2.1 Hard Sphere in Cylindrical Pore. 
having both hydrodynamic and Brownian characteristics; another is that the molecular size of solvent is much smaller than that of solute so that the solvent phase is treated as a continuum; and another is that the solute concentrations are very dilute; and the ratio of pore length to pore diameter is significantly large. The physical situation under consideration is depicted in Figure 2.1. The equation describing the transport of large molecules in the pore (based on these assumptions) is derived by balancing the diffusional force with hydrodynamic force acting on the molecule. This equation is express $2 d$ as:

$$
-\frac{k T}{C(r, z)} \frac{\partial c(r, z)}{\partial z}-6 \pi \mu a F(\lambda, \beta)(U-G(\lambda, \beta) V(r))=0
$$

The first and second terms in this equation represent the diffusional force and hydrodynamic force, respectively. Enhanced drag (ratio of pore to bulk friction coefficient) and lag coefficient arising from the difference between the velocity of solute and that of bulk fluid are denoted by 'F' and ' $G$ ' in the second term of Equation 2.6. Equation 2.6 leads to the stokes-Einstein equation $\left(D_{0}=k \cdot T /(6 \pi \cdot \mu \cdot a)\right)$ with the assumptions that solute movement is unbounded $(F=1)$ and fluid is stagnant $(\mathrm{V}=0)$.

By assuming no bulk fluid movement $(\mathrm{V}=0)$ and rearranging Equation 2.6 by the use of the Stokes-Einstein equation, Equation 2.7 is derived as: 


$$
U=-D_{-} \frac{F^{-1}(\lambda, \beta)}{c(r, z)} \frac{\partial c(r, z)}{\partial z}
$$

Thus, axial solute flux $\mathrm{N}$ is given by

$$
N=-D_{-} F^{-1}(\lambda, \beta) \frac{\partial c(r, z)}{\partial z}
$$

With this flux equation along with consideration of the steric restriction in radial variation of concentration, the average flux over pore cross section is expressed as:

$$
\langle N\rangle=-2 D_{-} \int_{0}^{1-\lambda} F^{-1}(\lambda, \beta) \cdot \frac{\partial c(r, z)}{\partial z} \cdot \beta d \beta
$$

The concentration term in Equation 2.9 is a local concentration. To relate the average flux over pore cross section, $<N>$, to the average concentration over pore cross section, $\langle c\rangle$, the concentration term is separated into the axial dependence term and the radial dependence term as shown in Brenner's and Gaydos' work (40). This is expressed as:

$$
c(r, z)=g(z) \exp \left[\frac{-E(\beta)}{k T}\right]
$$

Here, the energy potential, $E(\beta)$, define interactions between the solute and the pore wall. By taking the integration of Equation 2.10 over all radial position within the pore with the steric consideration, the local cross section average concentration is obtained as Equation 2.11 . Then, the local diffusional flux equation is expressed as Equation 2.12 . 


$$
\begin{aligned}
& \langle C\rangle=2 g(z) \cdot \int_{0}^{1-\lambda} \exp \left[\frac{-E(\beta)}{R T}\right] \beta d \beta \\
& \langle N\rangle=-D_{-} \cdot \frac{\int_{0}^{1-\lambda} F^{-1}(\lambda, \beta) \cdot \exp \left[\frac{-E(\beta)}{R T}\right] \beta d \beta}{\int_{0}^{1-\lambda} \exp \left[\frac{-E(\beta)}{R T}\right] \beta d \beta} \cdot \frac{d\langle C\rangle}{d z}
\end{aligned}
$$

Equation 2.12 can be further simplified as:

$$
\langle N\rangle=-K_{r} D_{-} \frac{d\langle c\rangle}{d z}
$$

where,

$$
K_{r}=\frac{\int_{0}^{1-\lambda} F^{-1}(\lambda, \beta) \cdot \exp \left[\frac{-E(\beta)}{R T}\right] \beta d \beta}{\int_{0}^{1-\lambda} \exp \left[\frac{-E(\beta)}{R T}\right] \beta d \beta}
$$

Here, $K_{r}$ is the area averaged intrapore mobility ratio of solute and the quantity $K_{r} D_{0}$ is an area averaged intrapore diffusion coefficient. Also, $<N>$ is the steady state flux based on the solute concentration inside the pore at $z=0$ and $z=1$. To relate the $f l u x<N>$ to the corresponding external solution, Equation 2.13 is integrated over pore length with the steady state assumption, so that:

$$
\langle N\rangle=-k_{\mathrm{r}} D_{-}\left(\left\langle C_{0}\right\rangle-\left\langle C_{L}\right\rangle\right)
$$

If $g(0)=C_{n}$ and $g(1)=C_{1}$, then, from Equation 2.11, the partition coefficient at the entrance and exit of the pore can be calculated as:

$$
K_{p}=\frac{\left\langle c_{0}\right\rangle}{c_{0}}=\frac{\left\langle c_{1}\right\rangle}{c_{1}}=2 \int_{0}^{1-\lambda} \exp \left[\frac{-E(\beta)}{k T}\right] \beta d \beta
$$


As a special case in calculating the partition coefficient $K_{p}$ only steric interactions between the solute and the pore wall are considered $(E(\beta)=0)$. Then, Equation 2.16 becomes:

$$
K_{p}=2 \int_{0}^{1-\lambda} \beta d \beta=(1-\lambda)^{2}
$$

This is the same form obtained by Ferry (35) and later by Giddings et al.(41). Equation 2.17 for the partition coefficient is developed for rigid spherical solutes with only steric considerations.

By applying Equation 2.16 to Equation 2.13, the flux equation, expressed in terms of bulk solution concentrations, is obtained as:

$$
\langle N\rangle=-K_{I} K_{p} D_{-}\left(C_{0}-C_{L}\right)=D_{\theta}\left\langle C_{0}-C_{L}\right\rangle
$$

and

$$
\frac{D_{\theta}}{D_{\infty}}=K_{r} K_{p}=2 \int_{0}^{1-\lambda} F^{-1}(\lambda, \beta) \cdot \exp \left[\frac{-E(\beta)}{R T}\right] \beta d \beta
$$

The Renkin equation, shown in Equation 2.5, can be derived from this expression by using Equation 2.17 for the partitioning effect, and by using the hydrodynamic factor obtained from the centerline approximation method $(\beta=0)$.

Rigorous derivation of analytical expressions for hydrodynamic coefficient $K_{r}$ requires complex mathematical manipulation as shown in Brenner's and Gaydos' work (40). Yet, the hydrodynamic coefficients of different shaped models other than a sphere model in a cylindrical pore were 
not avalable in up-to-date literature. Meanwhile, the partition coefficient term, $K_{p}$, has been study quite extensively for the various shapes of molecules in different shapes of pores $(41,103,100)$. For example, Giddings et al. (41) reported the partition coefficients of solutes having different shapes, such as disc and rod shapes, in inert pores $(E=0)$ determined from the statistical mechanics method. In their study, they showed that the mean external length (or mean projected length), the closest distance of the molecule's center to a flat surface averaged over all orientations, characterized the partitioning better than any other size parameters such as radius of gyration, equivalent hydrodynamic radius and molecular weight. An experimental study recently conducted by Limbach et al. (99) also reported that the molecular size was best described by the mean projected molecular radius for partitioning in the hindered diffusion experiment of a rigid disk-shaped molecule.

The effect of interactions $(E \neq 0)$ on the partitioning of solute was investigated both theoretically $(106,107)$ and experimentally $(25,31)$. Davidson et al. (100) conducted a partitioning study for a flexible polymer using the Monte Carlo simulation technique, and reported that the introduction of small, attractive interaction between polymer and pore wall produced dramatic increases in the partition coefficients of a solute. 
Further treatment of theoretical developments is beyond the scope of current study. Instead, results of some theoretical studies are introduced in this section. Brenner and Gaydos (40) proposed an expression of $K_{r} K_{p}$ which is different from the widely used Renkin equation for $\lambda<0.1$ in their theoretical study. The equation is expressed as:

$$
\frac{D_{\theta}}{D_{-}}=K_{r} K_{p}=1-\left(\frac{9}{8}\right) \lambda \ln \lambda^{-1}-1.539 \lambda+o(\lambda)
$$

For large $\lambda$, Mavrovouniotis and Brenner (43) obtained the following expression:

$$
\frac{D_{\theta}}{D_{\infty}}=0.9841(1-\lambda)^{\frac{9}{2}}
$$

\section{Experimental studies in Hindered Diffusion}

In many experimental diffusion studies, evidence of hindered diffusion was investigated with both melubranes and catalytic support materials. Results of these studies were mostly evaluated in the light of the hydrodynamic theories reviewed in the previous section.

In general, the application of the hydrodynamic theory to interpret the experimental results in the previous diffusion studies with catalyst support material carried some ambiguity because the structures of catalyst supports were relatively complex, and diffusing solutes were susceptible to interact with support materials. The same 
ambiguity also existed in diffusion studies which used membranes other than track-etched ones due to the uncertainty coming from membrane structures. However, several studies either with catalyst support materials $(45,48,49,99)$ or with membranes $(38,50)$ reported the agreement of data with that predicted by Renkin's equation or other approximate estimation equations. Among these studies, it is interesting to note that results from similar diffusion experimental systems showed inconsistencies in agreement of experimental data with theoretical estimations of colton (44) and Bishop (45) with catalytic support materials, and of Conlon (46) and Cannell (47) with tracketched polycarbonate membranes.

In the diffusion studies with track-etched membranes, the disadvantages of complex geometry of the previous transfer media were eliminated by careful characterization of the membranes. Thus, the results of the experimental hindered diffusion studies could be directly evaluated from the theoretical standpoint. Detailed descriptions of some experimental hindered diffusion studies relevant to the current research are as follows.

Ackers and steere (50) studied the hindered diffusion of human hemoglobin and southern bean mosaic virus particles through a thin agar membrane. They analyzed the observed results with Renkin's equation and reported close agreement. 
Conlon and craven (46) measured the hindered diffusion of various ions and molecules in the pores of a membrane filter. In one of their experiments, they used the Nuclepore ${ }^{T M}$ polycarbonate membrane to measure the effective diffusion coefficients of polymer spheres through pores. The results were correlated against the ratio of solute to pore radius and were compared with predicted results from Faxen's expression (36), Ferry's steric correction (35), and Renkin's equation. They reported that experimental results were lower than those predicted by these theories, and proposed a new theory based on the transition state theory to explain their observations.

However, Beck and Schultz (54) showed that Renkin's expression agreed with their experimental data. In their experiment, track-etched mica membranes were prepared by a process of bombardment with fission fragments from a $U^{235}$ source and a subsequent etching with hydrofluoric acid. The effective diffusivity of various-sized solutes with diameter-to-pore ratios ranging from 0.0086 to 0.3784 were measured in an aqueous solution.

More and Katzer (55) conducted counterdiffusion experiments with liquid hydrocarbons in type $Y$ zeolite and investigated the effect of molecular size, type and direction of diffusion. The presence of a diffusional limitation affects the activity of a zeolite catalyst. They measured the effective diffusion coefficient for the 
counterdiffusion of hydrocarbons, and observed a distinct effect of molecular size on the effective diffusion coefficient. Although this system was somewhat different from the membrane diffusion in terms of adsorption and desorption of solute molecules, their work established correlations between the effect of molecular size and the molecule-zeolite interactions on the diffusion rate.

A similar diffusion experiment using catalysts to examine the effects of molecular size was performed by satterfield et al. (56). They used homogeneous silica alumina bead catalysts, a variety of systems of paraffins and aromatic hydrocarbons, and various aqueous sugar and salt solutions. Two important findings were reported in their study; for nonadsorbing molecules such as saturated hydrocarbons or aqueous sugar solutions, the equilibrium steric partition factor was close to unity; and the logarithm of the ratio of effective to bulk diffusivity decreased linearly with an increasing ratio of solute to pore radius. The authors reported that the predicted equations derived from the hydrodynamic theory were not applicable to their system. Also, they noted the evidence of surface diffusion in their experimental system with some aromatic hydrocarbon solutes.

colton et al. (44) studied the diffusion of macromolecules by using fine, porous glass cubes having narrow pore size distribution. Polystyrenes, having 
molecular weights ranging from 600 to 67,000 , and two proteins were used to measure the effective diffusivities and the partitioning factors. In this study, the equilibrium partitioning factors decreased with the increase of the ratio of solute to pore radius for all solutes. The restricted diffusion effects found for all protein molecules were consistent with predicted models. However, these effects were not observed with polystyrenes.

They reported that the result was attributed to the molecular configuration of polystyrene within pores, and was termed a particle drain effect.

Wong and Quinn (57) studied hindered diffusion using the bovine serum albumin (BSA) as a diffusing molecule through the track-etched mica membrane. They measured the steric partitioning factor and restricted the diffusion rate separately to determine the overall effect.

To measure the steric partitioning factor, the pore wall was precoated with a high molecular weight polysaccharide to preclude protein adsorption. Pore conductivity was then measured in the presence and absence of BSA. The difference in conductivity implied a concentration difference of BSA. The steric partitioning factor was calculated accordingly. The diffusion rate was measured by using a fluorescent tag to monitor the steady state flux of BSA through a membrane of known pore characteristics. They reported that the observed results of 
the hindrance effect were twice that predicted by Renkin's equation.

Malone and Anderson (25) investigated the effect of the pore wall and diffusing solute interactions produced by electrostatic effects on hindered diffusion. Latex particle diffusion through a track-etched mica membrane was measured as a function of pore radius and solution ionic strength. To study the electrostatic effect on transport rates, latex particles were stabilized with an anionic surfactant. Observed results showed much lower diffusivity than that predicted by the hydrodynamic theory. They attributed this result to the electrostatic repulsion between particles in pores, thus reducing the concentration driving force. They also reported that ionic strength controls this interaction through Debye's ionic strength parameter. The authors proposed a modified equation to the hydrodynamic theory which accounts for these interaction parameters and shows agreement with their experimental results.

Prasher et al. $(58,59)$ studied the effects of adsorption equilibrium and ratio of solute to pore diameter on the liquid phase effective diffusivity. Alumina pellets with a diameter of about $0.32 \mathrm{~cm}$ were used with various pairs of solute-solvent systems. Two experiments were conducted: a transient diffusion experiment measuring effective diffusivity, and an equilibrium adsorption measurement. The results obtained in their study showed 
that the effect of the partitioning factor was significant on the effective diffusivity. They also showed that the ratio of diffusivity in the pore to that in the bulk solution, multiplied by the partitioning factor, was wellcorrelated with the ratio of solute diameter to pore diameter by a semi-log plot. This correlation was consistent with that of Satterfield et al. (56), which showed disagreement with the Renkin equation. In Satterfield's work, however, the partitioning factor was assumed to be unity, while in Prasher's and Ma's work, it was considered to be important.

Glandt (60) postulated the pore-bulk partitioning factor for solute molecules in a virial-type expression as a series in the power of bulk concentration. This work addressed the effect of solute-solute interactions within pores to explain the experimental value of the partitioning factor, which was larger than the preaicted value from a simple steric exclusion expression.

Another study of restrictive diffusion in which adsorption was taken into account was performed by chantong et al. (48). They used activated alumina and polycyclic aromatic hydrocarbons having different sizes, and measured the effective diffusivities of these molecules in catalysts. They obtained both the Freundich isotherm from equilibrium adsorption experiments and the effective diffusivity of model compounds by treating the kinetic data of diffusion 
experiments using a pore diffusion model with the Freundich isotherm. Results showed a decrease in effective diffusivity in the order of increasing ratio of molecule-topore radius. The authors proposed a correlation equation between the ratio of effective diffusivity to bulk diffusivity and the ratio of molecule radius to pore radius. This expression was similar to the correlation expression from previous studies using catalyst particles as a diffusion medium (Satterfield (56), Prasher and Ma, (58)). They also found that the contribution of surface diffusion to intraparticle diffusion was insignificant in their experimental system.

Cannell and Rondelez (47) measured the effective diffusivity of polystyrene having weight-average molecular weights of 110000,233000 , and 600000 with narrow molecular weight distributions. Ethyl acetate was used as a solvent, and Nuclepore ${ }^{T M}$ polycarbonate membranes were used in these experiments. The hydrodynamic radii of large mojecules were calculated from the stokes-Einstein equation. Results from these experiments correlated well with the Renkin's equation with the ratio of molecule-to-pore radius multiplied by a factor of 1.45 . The factor, 1.45 accounted for the characterization of polymer coils when a hydrodynamic radius was used. They also examined the effect of concentration on effective diffusivity, and reported that flux enhancement was observed. 
The concentration dependence of enhanced diffusion was also investigated by Gillot et al. (30). Experiments were conducted using polystyrene molecules as diffusing solutes across track-etched polycarbonate membranes. They reported that concentration dependence of the partition coefficient was attributed to this enhanced diffusion.

Deen, Bohrer, and Epstein (26) studied the effects of molecular size and configuration on hindered diffusion. To examine the configurational effect, they used polysaccharide with different molecular structures. Diffusion experiments were carried out with these molecules at ratios of solute diameter to pore diameter as high as 0.77 by using Nuclepore ${ }^{T M}$ polycarbonate membranes. Their results showed that dextran, having a nearly linear structure, showed less hindrance than ficoll, having a crosslinking structure, over the entire range of molecular sizes studied. They attempted to explain these observations by referring to the particle drain effect stated by colton et al. (44), and to dextran's ability to deform. The authors also reported that ficoll behaved more like the rigid sphere model, thus showing effective diffusivities close to predicted values from a hydrodynamic model, although these values were somewhat less than predicted.

Similar experiments performed by Bohrer and his coworkers (28) confirmed these results. These two studies indicate that molecular configuration, or the ability to 
change configuration, is an important factor in the determination of effective diffusivity.

Kathawalla et al.(42) also verified the configurational effects of molecules in a hindered diffusion study. They examined the configurational effect of two types of molecular geometry: planar disklike molecules (porphyrin) and wormlike molecules (short-chain polystyrene). They reported that the diffusion of short-chain polystyrene was more hindered than that of porphyrin, both with equivalent ratio of stokes-Einstein radius to pore radius.

Diffusion coefficients of petroleum asphaltenes at hindered diffusion conditions were investigated by two research groups (51-53). Asphaltenes, being defined as a solubility class, are complex mixtures of heavy hydrocarbons and heteroelements, and are not well-characterized substances. In the experiment conducted by Baltus et al. (29), asphaltene was extracted from Kuwait atmospheric bottom using a criteria of n-pentane insolubility and tetrahydrofuran solubility at $25^{\circ} \mathrm{C}$. Effective diffusivity of the whole asphaltene across the track-etched mica membrane was examined in a tetrahydrofuran solution. In their experiments, polystyrene molecules of various sizes were used as an internal calibration standard of the size of asphaltene fraction. They reported that measured diffusion coefficients were in agreement with those predicted from the hydrodynamic theory of hindered diffusion. 
Sane and Isotsis (16) also investigated effective diffusivities of petroleum asphaltenes across track-etched polycarbonate membranes. These invertigators observid that the diffusion of asphaltenes was hindered, and reported that the degree of hindrance depended on the relative sizes of asphaltene fragments to membrane pore sizes.

The review of previous experimental studies revealed that many of the experimental results confirmed the validity of the hydrodynamic theory reviewed in the previous section. However, a substantial number of studies did not agree with the theoretical predictions, and in fact some studies showed conflicting results. Thus, much additional work is needed to completely understand the phenomenon of hindered diffusion.

\section{Track-etched Membranes}

The track-etching process, discovered by Price and Walker $(61,62)$ and subsequently developed by Bean (63), provides a way of obtaining membranes with uniform pores for hindered diffusion experiments. These membranes are characterized by a uniform distribution of pore sizes with known pore geometry and nearly identical cylindrical holes. Pore density and pore length can easily be determined, and consequently all information about the membrane can easily be retrieved.

Track-etched membranes can be made from various materials, including polycarbonate, polyester and mica. 
Some polymeric membranes are commercially available from the Nuclepore Corporation (Pleasanton, California). These membranes have many areas of practical application, such as blood cell separation, environmental study of particulates in the atmosphere and ocean, sampling of radioactive aerosols, and microbiology separations.

The basic principles in the formation of track-etched membranes are as follows. The traversing of heavily charged, nuclear particles through thin solid films leaves a damaged path on an atomic scale called tracks. Radioactive materials or heavily charged particles can be used as trackforming particle sources. Fission fragments are especially good for this purpose. Spontaneous fission of very large mass number isotopes produces a large number of energetic, heavily charged particles. A widely used isotope is $\mathrm{Cf}^{252}$ (Californiuin). Theoretically, any dielectric material may be track-etched whether an inorganic crystal or a longchain polymer (62). In the crystal, the track is formed by the particles causing the structural disorder of lattice. In polymer, the track is produced by the particles breaking chemical bonds between atoms in a long-chain molecule's chemical bondage. These damaged tracks are easily and selectively attacked by a properly chosen strong reagent, which forms the etched holes. Hole sizes can easily be controlled by varying the etching time at a constant temperature and concentration of etching agent. 
studies on the etching characteristics of fission tracks in various material were conducted by several researchers $(64-68)$. According to these papers, the etching rate depends on the etching temperature, the concentration of etching reagent, and pretreatment of irradiated films (ultraviolet light treatment or exposure to air).

\section{Coal Asphaltene}

Coal asphaltene is a generic term describing a complex mixture of coal molecules which are soluble in aromatic hydrocarbon solvents, such as benzene or toluene, but insoluble in certain saturated hydrocarbons, such as pentane or hexane. Thus, the characteristics of one coal asphaltene may be somewhat different from that of other coal asphaltene, depending on the origin uf the coal from which the asphaltene is derived. Many studies have been conducted to characterize coal-derived asphaltenes. Steedman (13) provided a relatively comprehensive review of these papers. The term 'asphaltene' originally was used to describe a petroleum sub-fraction, and now has been extended to depict a fraction of coal-derived material. However, there are big differences between these two materials. Several studies have been reported on a comparison of these two asphaltenes. $(69,70)$. One result of these studies, conducted by Yen (69), is provided in Table 3.2. As shown in this table, a major difference between the two asphaltenes is the average molecular weight; coal asphaltenes have a considerably lower 
number averaged molecular weight and are more highly functionalized. Other major differences reported in this study were as follows; The aromaticity of coal-derived asphaltene $\left(f_{a}=0.6-0.7\right)$ is higher than that of petroleumderived asphaltene $\left(f_{a}=0.2-0.5\right)$; the aromatic ring systems within petroleum-derived asphaltenes are much more condensed $\left(\mathrm{H}_{\mathrm{ar}} / \mathrm{C}_{\mathrm{ar}}=0.3-0.5\right.$, which is peri) than those of coal-derived asphaltene $\left(\mathrm{H}_{\mathrm{ar}} / \mathrm{C}_{\mathrm{ar}}=0.5-0.7\right.$, which is kata); the substituents of the petroleum-derived asphaltenes are longer $(n=4-6)$ than those of coal-derived asphaltenes $(n=1-2)$; and the aromatic system of petroleum-derived asphaltenes is extensively substituted (50\%-70\%), whereas the coal-derived asphaltene is sparingly substituted $(30 \%-50 \%)$.

A typical structure of coal asphaltene was proposed by several groups. In Figure 2.2, a hypothetical crosssectional view of a coal asphaltene model proposed by Yen (122) is presented. According to this model, asphaltene particles consist of sheets of condensed aromatic rings coupled with saturated side chains and naphthenic rings. Coal molecules in asphaltene are strongly associated by the $\pi-\pi$ bonds (bond strength: ca. 10-15 Kcal/mole) existing between condensed aromatic rings and by the hydrogen bonds (bond strength: ca. $5 \mathrm{Kcal} / \mathrm{mole}$ ) occurring between acidic functionalities and basic functionalities.

This structural information is important in the current study since the hindrance factor is also highly dependent on 
the configurational factor of molecule. Regardless of the exact structure of these macromolecules, it is evident that most asphaltene molecules will encounter so-called configurational, hindered, or restricted diffusion in the catalyst pores because of the size surmised from the molecular weight, and because of the fact that the pore sizes in catalyst pellets are between 20-200 \& (15). 
Table 2.2

\section{Comparisons of Properties Between Petroleum-and Coal-Derived Asphaltenes (69)}

\begin{tabular}{|c|c|c|}
\hline Property & $\begin{array}{l}\text { Coal } \\
\text { Asphaltenes }\end{array}$ & $\begin{array}{l}\text { Petroleum } \\
\text { Asphaltenes }\end{array}$ \\
\hline $\begin{array}{l}\text { Number-average molecular } \\
\text { weight }\end{array}$ & $400-800$ & $2000-20,000$ \\
\hline Weight percent carbon & $84-89$ & $80-86$ \\
\hline Weight percent hydrogen & $5 \cdot 8-7 \cdot 3$ & $7.7-9.3$ \\
\hline Weight percent oxygen & $2-6$ & $1-2.7$ \\
\hline Weight percent nitrogen & $1-3$ & $0.3-0.5$ \\
\hline Weight percent sulfur & 0.1 & $3-9 \cdot 3$ \\
\hline $\begin{array}{l}\text { Percent ash by low or } \\
\text { high temperature }\end{array}$ & $<0.1$ & $<2$ \\
\hline $\begin{array}{l}\text { Aromaticity, by } \\
\text { NIIR }\end{array}$ & $0.6-0.7$ & $0.4-0.55$ \\
\hline $\mathrm{H} / \mathrm{C}$ & 0.94 & 1.12 \\
\hline Forms of oxygen & $\mathrm{OH},-\mathrm{O}-$ & $\mathrm{OH},-\mathrm{C}=\mathrm{O},-\mathrm{O}-$ \\
\hline Forms of nitrogen & & \\
\hline Metals & $\begin{array}{l}\text { iron, } \\
100-300 \mathrm{ppm} \\
\text { titanium, } \\
20-50 \text { ppm }\end{array}$ & $\begin{array}{c}\text { vanadium, } \\
100-400 \mathrm{ppm} \\
\text { nickel, } \\
50-150 \mathrm{ppm}\end{array}$ \\
\hline
\end{tabular}


$\left.x_{1}\right) \stackrel{\text { sheel }}{\longrightarrow}$

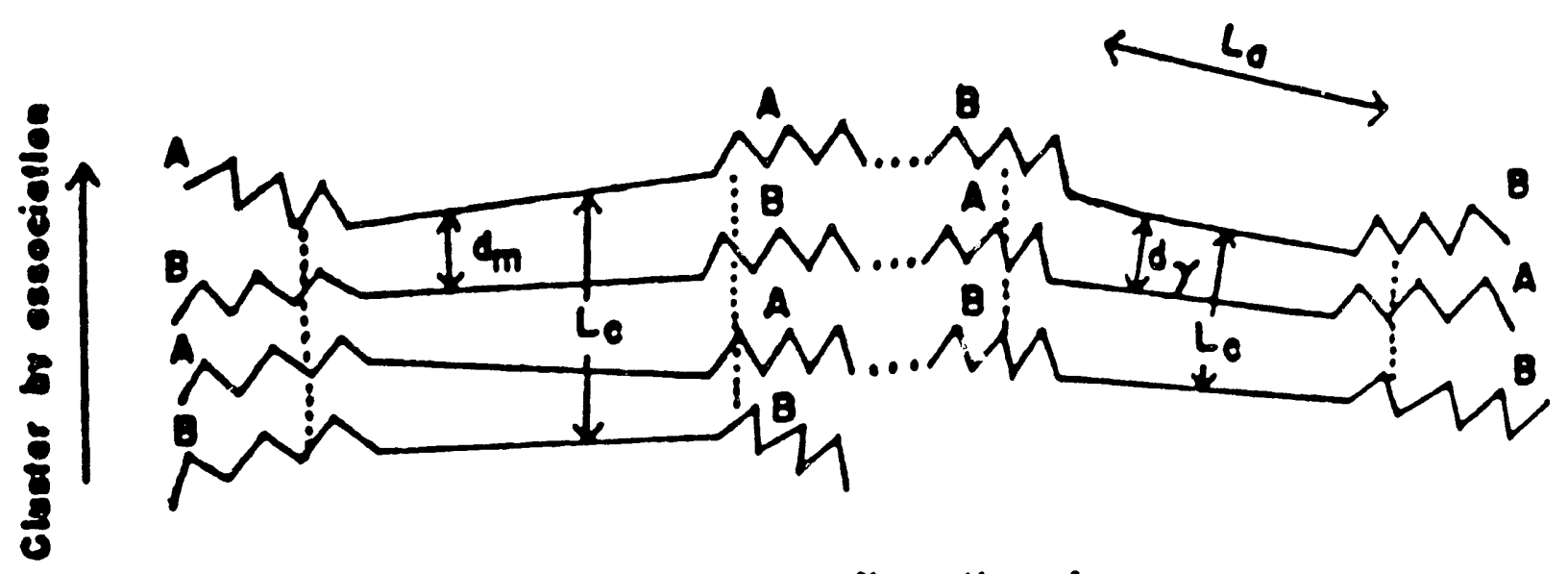

M zlg-zog conflqurotion of
seluroped corbon choins
or nopthenle rings - idge of condensed oromatle

....... H-bonding or chorge-Ironsfor bonding

A oetd

- base

Figure 2.2 Hypothetical Cross-sectional view of coal Asphaltene Proposed by Yen (122) 


\section{PREPARATION AND CHARACTERIZATION OF MEMBRANES USED FOR HINDERED DIFFUSION STUDY}

\section{Preparation of Membranes for Hindered Diffusion study}

The average molecular weight of coal asphaltene is about 400-800 as shown in Table 2.2. For the experimental study of hindered diffusion with molecules having these molecular weights, the pore sizes of membranes which cause appreciable hindrance effects would be around 100-200 \& (the longest axial length of rubrene $(\mathrm{MW} ; 607$ ) is about $16 \AA$ (108)). Unfortunately, the pore sizes of commercial tracketched membranes did not have pore sizes suitable for the hindered diffusion study of coal macromolecules; although the smallest nominal pore size of a commercial track-etched membrane was $150 \AA$, actual pore sizes of these membranes were about two times higher than this nominal value as described later in this chapter. Thus, efforts were made to prepare membranes appropriate for the current hindered diffusion study.

In order to make the membranes, polycarbonate films and polyester films irradiated with $\mathrm{Cf}^{252}$ fission fragments were obtained from the Nuclepore Corporation (Pleasanton, CA), and the etching characteristics of these films were 
investigated. Also, an attempt was made to prepare alumina membranes by using of the anodic oxidation technique. It was reported that the characteristics of alumina membranes were similar to those of track-etched membranes (71). The pores in alumina membranes are straight, uniform and nearly cylindrical. Thus applicability of alumina membranes to diffusion research may be much better than that of organic track-etched membranes, because of their inertness.

\section{Polycarbonate Membranes}

Previously, De Sorbo (65) conducted an extensive study of the etching characteristics of polycarbonate films irradiated by fission fragments of $\mathrm{Cf}^{252}$. Guillot and Rondelez (67) also studied etching characteristics of tracks in polycarbonate film. They used high-energy krypton ions $\left(\mathrm{Kr}^{+}\right)$to produce tracks in polycarbonate films. In addition, there have been several studies concerning the etching characteristics of fission tracks in various materials, including polycarbonate films $(62,63,64,73)$.

Although several studies had been carried out in the literature, the results of these studies gave only qualitative information regarding the etching rate of the polymer films used in the current research due to the differences in the properties of films. Thus, the results were not directly applicable to the current study

In this research, the optimum process conditions for the etching to make membranes suitable for the hindered 
diffusion study were investigated by the use of irradiated polycarbonate films.

\section{Experiments}

Two sets of irradiated polycarbonate films were purchased from the Nuclepore Corporation (Pleasanton, CA). one set of film of dimension $3^{\prime} x 3^{\prime}$ was aged at atmospheric room conditions for more than 6 months after purchase. The other set of films, with dimensions of 8" X 11", was used without aging. Instead, some of these films were treated with ultraviolet (UV) light; each side of a film was exposed to UV light for 2 hours before etching. Previous studies reported that UV treatment of irradiated polycarbonate films had an effect on the etching rate of films which proved similar to a long period of aging $(65,67)$.

The values of track density and thickness of the films, specified by the manufacturer, were $6 \times 10^{8}$ tracks $/ \mathrm{cm}^{2}$ and $6 \mu \mathrm{m}$, respectively. These values were used in the calculation of pore sizes without further measurements.

circular specimens of $47 \mathrm{~mm}$ in diameter were cut from original polycarbonate films and clamped between two washers made from Gylon ${ }^{\text {TM }}$ gaskets with binder clips. The specimen assemblies were then washed with a cleaning solution ( 1 wt\% Alconox ${ }^{T M}$ detergent solution) and rinsed with distilled water to remove any contaminants on the surface of the film. The etching of these specimens was conducted in caustic 
solution contained in a jacket cooled beaker. I, 0.01 wt Dowfax $2 \mathrm{Al}$ surfactant (Dow Chemical Co.) was added to the caustic solution to improve the wettability of film. The temperature of etching solution was controlled to $\pm 0.1{ }^{\circ} \mathrm{C}$ by a constant temperature water circulating bath, After a preset time for etching, the specimen was taken out and quickly rinsed with distilled water, then washed thoroughly with pure water (Fisher scientific, optima grade). The temperature of the pure water was kept close to the freezing point of water to quench further etching reaction. Washing times were at least 3 hours, depending on the pore sizes of membranes produced. The specimen was then dried in a vacuum oven.

Pore sizes were determined by he gas permeability tests and diffusion experiments. Since the gas permeability method poses problems in measuring small-sized pore (less than 200 A), diffusivity measurements of n-octane in $\mathrm{n}$-decane were conducted to measure the pore sizes of membranes having small pores. Details of the obtaining pore diameters from the experimental data are described in the membrane characterization section of this chapter.

\section{Results and Discussion}

The effect of the etching time, concentration of caustic, and etching temperature were investigated in order to optimize etching conditions for membrane preparation. In Figure 3.1, the change in the calculated pore diameter as a 


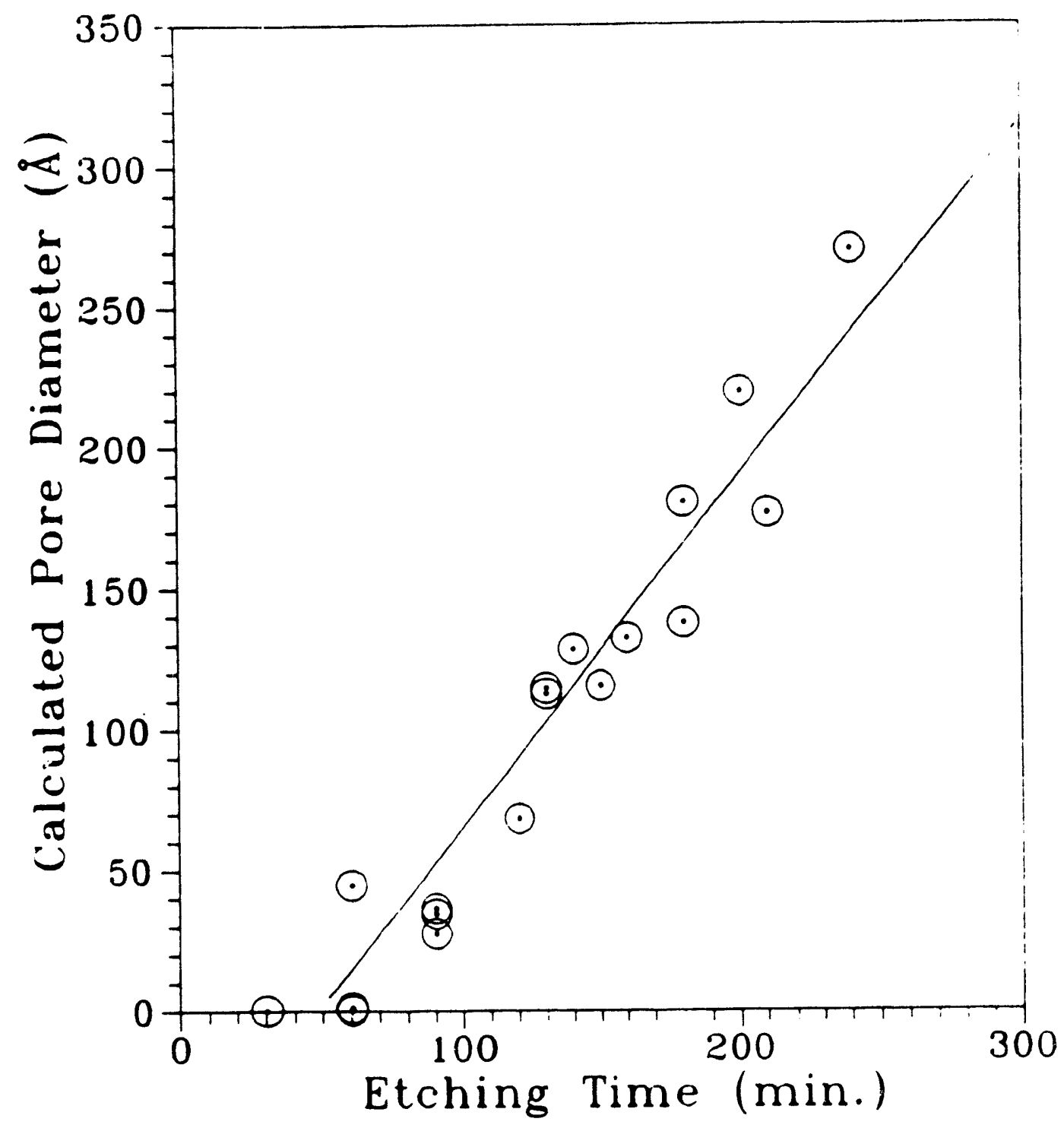

Figure 3.1 Etching Curve of Polycarbonate Films (2 $N$ NaOH sol'n, 0.01 wty Dowfax, (2 $\left.20^{\circ} \mathrm{C}\right)$. 
function of etching time is presented. This study was conducted with the aged polycarbonate films. The concentration of caustic solution and the temperature of etching solution were $2 \mathrm{~N}$ and $20^{\circ} \mathrm{C}$ respectively. In Figure 3.1, the data points for the pore sizes over $200 \AA$ were obtained from gas permeability tests, and other data points were obtained from diffusivity measurements of n-octane. As shown in Figure 3.1 , the first pore breakthrough appeared to take place in about 60 minutes, and the etching curve followed a nearly straight line.

The experimental results on the effect of caustic concentration on the etching rate are presented in Figure 3.2. In the study, unaged polycarbonate films without UV treatment were used and three different concentrations of caustic solution $(2 \mathrm{~N}, 4 \mathrm{~N}, 6 \mathrm{~N})$ kept at constant temperature, $20{ }^{\circ} \mathrm{C}$, were tested. The results show that the etching rates increase from $0.82 \mathrm{~A} / \mathrm{min}$ with the $2 \mathrm{~N}$ solution to $4.34 \mathrm{~A} / \mathrm{min}$ with the $6 \mathrm{~N}$ solution. Also, it was found that etching processes proceeded quite linearly with etching time.

The effect of etching temperature on the etching rate was examined at $10^{\circ} \mathrm{C}, 20^{\circ} \mathrm{C}$ and $30^{\circ} \mathrm{C}$. The same concentration of caustic solutions (4N) was used in each experiment. Unaged polycarbonate film without UV treatment was also used in this study. The results are presented in Figure 3.3 . Etching rates are found to be $0.40 \mathrm{~A} / \mathrm{min}$ at $10^{\circ} \mathrm{C}, 1.45 \mathrm{~A} / \mathrm{min}$ at $20^{\circ} \mathrm{C}$ and $4.0 \mathrm{~A} / \mathrm{Min}$ at $30^{\circ} \mathrm{C}$. From this figure, an 
51

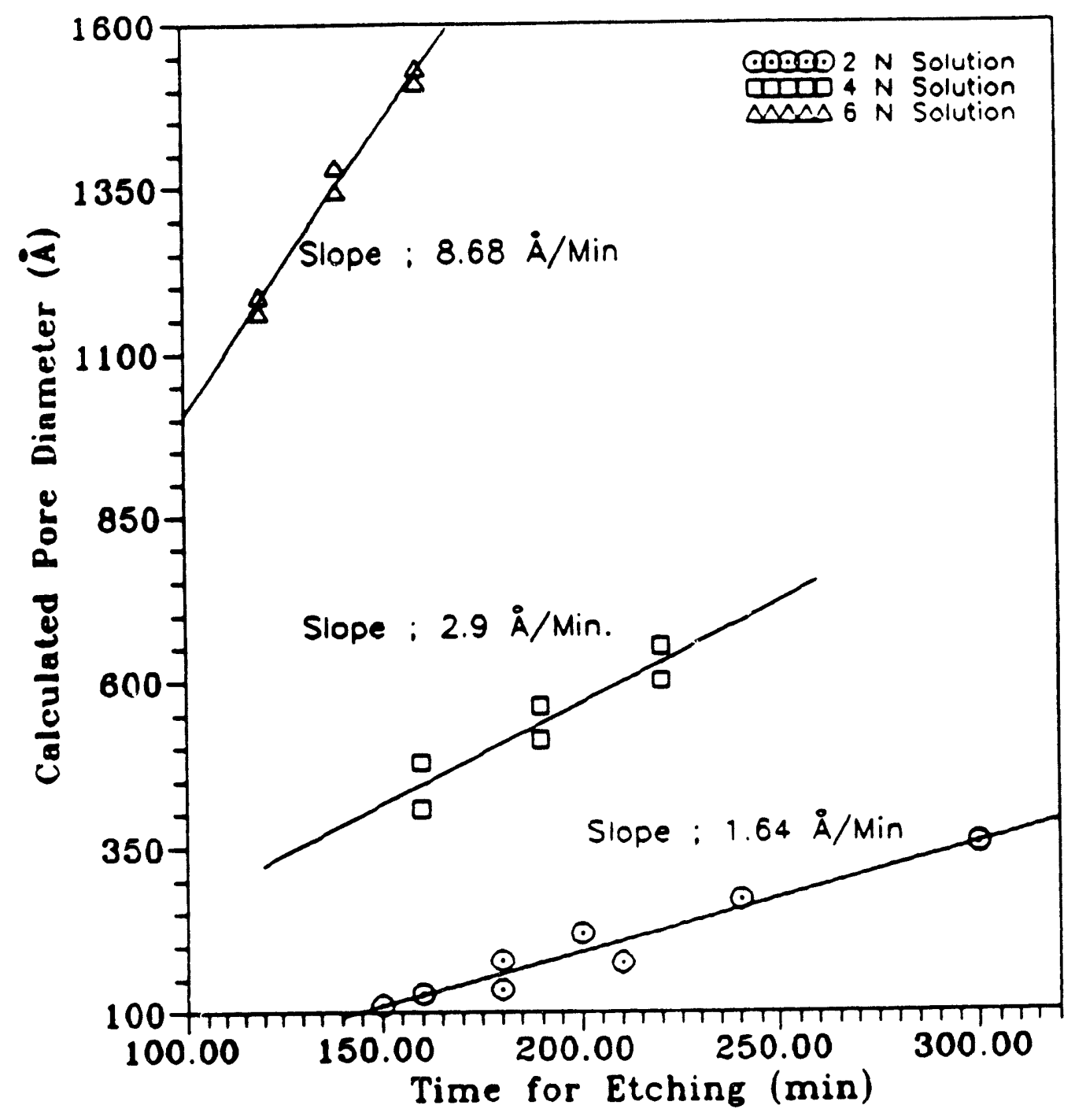

Figure 3.2 Effect of Caustic concentration on the Etching Rate 


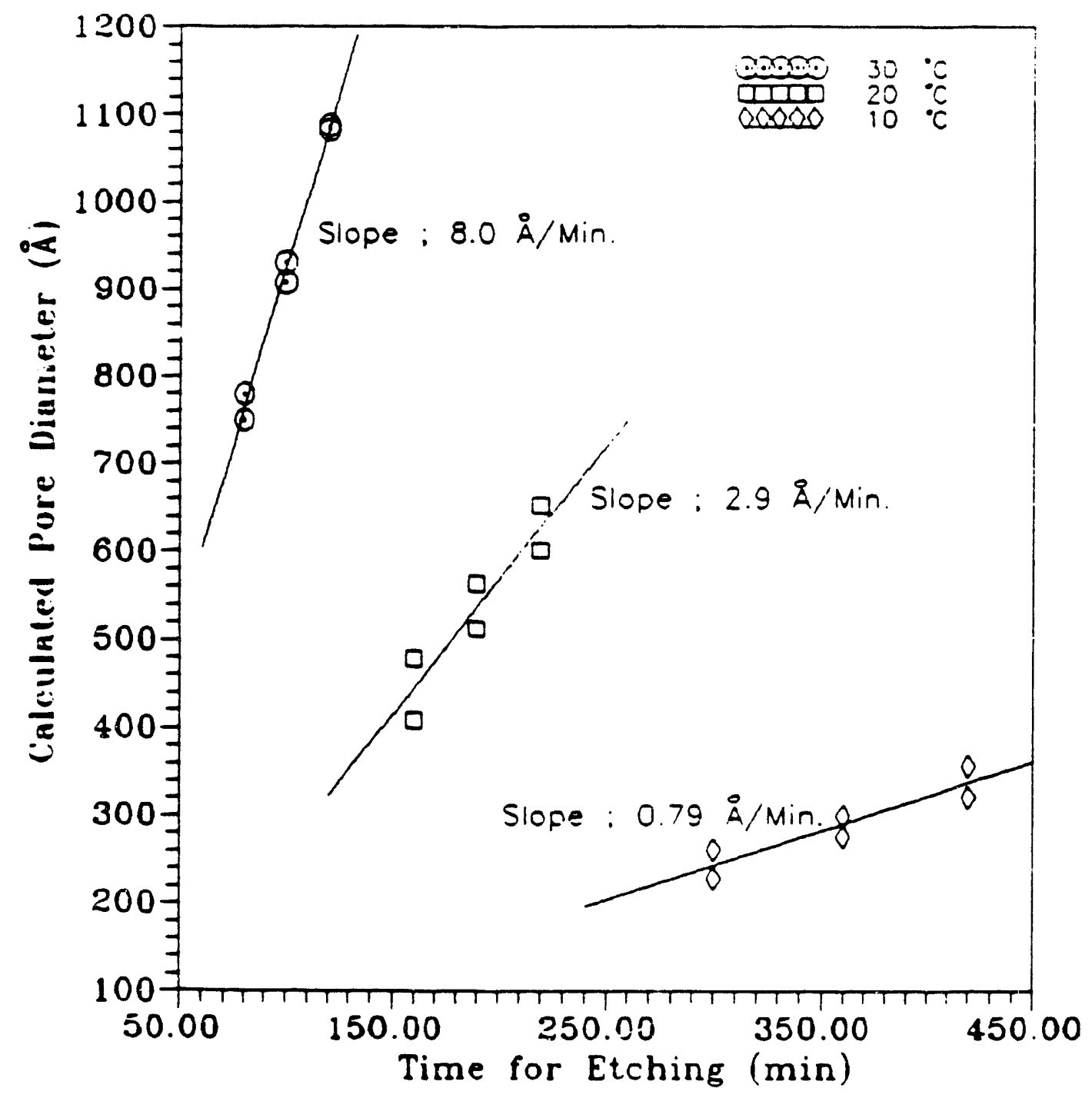

Figure 3.3 Effect of Temperature on the Etching Rate 


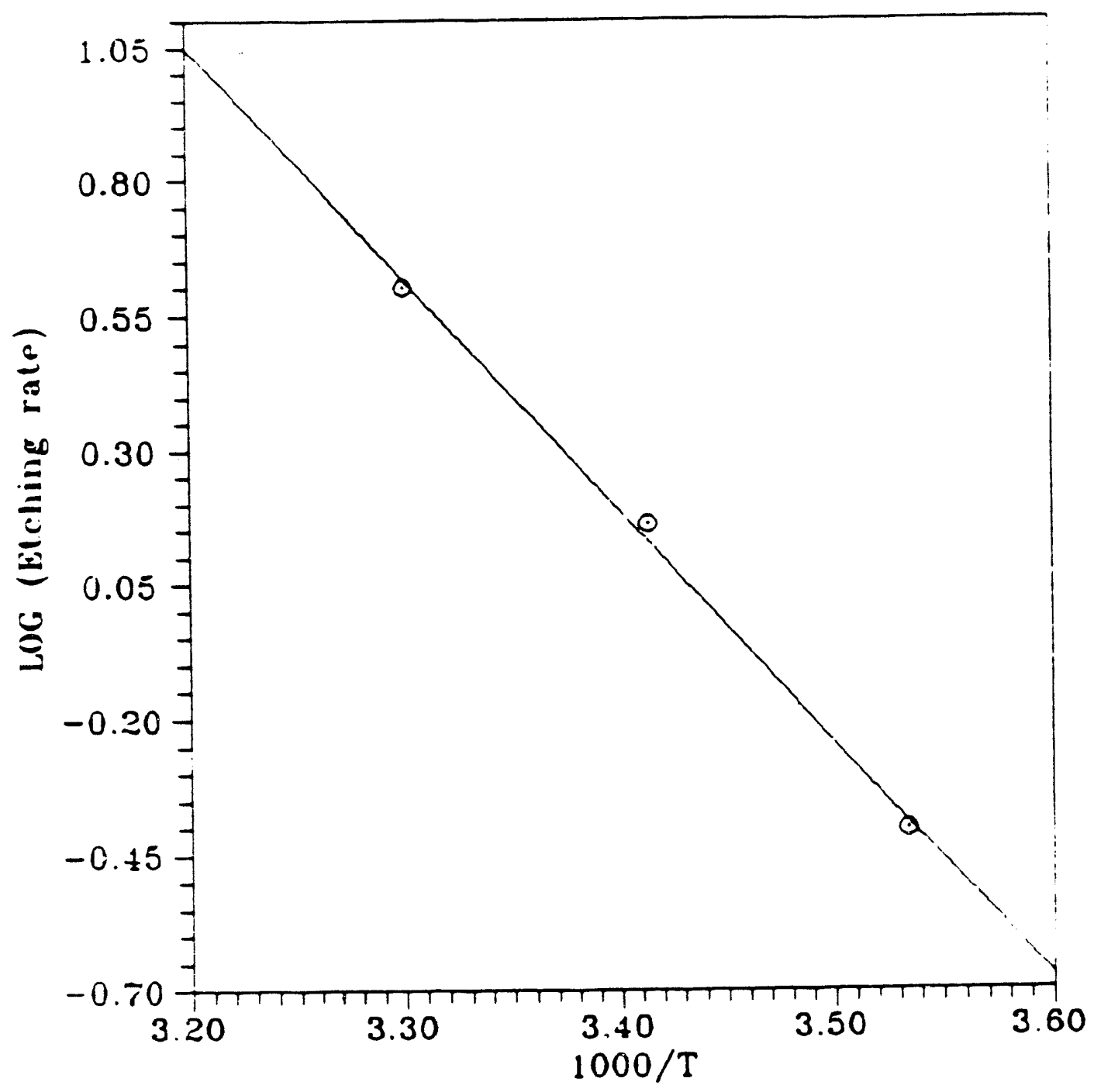

Figure 3.4 Arrhenius Plot of Etching Rates 
activation energy of the etching process was determined by plotting the etching rates at different temperatures in the Arrhenius plot. As shown in Figure 3.4, this value is found to be $0.78 \mathrm{eV}$. A similar study using $2 \mathrm{~N} \mathrm{NaOH}$ as an etchant reported this activation energy to be $0.66 \mathrm{eV}$ (67).

A conclusion from the etching study of polycarbonate film is that the etching rate strongly depends on both the concentration and the temperature of the etching solution. However, from an experimental standpoint, controlling the etching temperature is easier than controlling the concentration of etching solution when a small-size pore membrane is prepared, since the etching process can be more easily quenched with the control of temperature.

\section{Polyester Membranes}

Polycarbonate membranes had been used in several previous hindered diffusion studies where chemical compatibility of membranes with solvents might be questionable (e.g., ethyl acetate $(30,47)$ and xylene (52)). Actually, one study among these reported that the flux rate of ethyl acetate was always higher than that of water when the same polycarbonate membrane was used in the liquid permeability tests to determine the pore sizes of membranes (47). In the current study, similar results, which appeared to be caused by the chemical incompatibility of membranes, were observed in the diffusion experiments of $n$-octane with ethyl acetate using a polycarbonate membrane; in these 
experiments, a significant amount of n-octane flux was observed although the polycarbonate membrane used in the experiment had no pores. It appeared that this observation was due to the permeation of $n$-octane through membrane matrix (membrane body). Details of these experiments are described in Appendix c. Experimental results found in the current study as well as in the literature indicated that polycarbonate membranes were not suitable for the hindered diffusion study of ' macromolecules, since they were not fully chemically compatible with ethyl acetate which was adopted as a solvent for coal macromolecules (109).

Polyester membranes are another commercial membrane produced by the track-etched process. These membranes have much more chemical resistance to most solvents than polycarbonate membranes. However, the commercial polyester membranes available in the market have fairly large pore sizes (the smallest nominal pore size: $0.1 \mu \mathrm{m}$ ). Thus, these commercial membranes were not suitable for the hindered diffusion study either.

In an attempt to make membranes suitable for the hindered diffusion study of coal macromolecules, an etching study of irradiated polyester film was conducted.

\section{Experimental}

Irradiated but unetched polyester films were purchased from the Nuclepore Corporation. The track density and thickness in the specification provided by the company were 
$3 \times 10^{8} \mathrm{tracks} / \mathrm{cm}^{2}$ and $6 \mu \mathrm{m}$, respectively. The thickness of the membrane was checked by a weighing method. The density of a bulk polyester film (assumed as the specific gravity of poly 1,4-butylene terephthalate pellet: 1.31) was used to calculate the thickness. The average thickness of one polyester membrane calculated by the weighing method was $6.07 \mu \mathrm{m}$.

Etching procedures were the same as those used in the etching of polycarbonate film. Each side of the film was exposed to UV light for 2 hours before etching. A IN NaOH solution with 0.02 wt? Dowfax ${ }^{T M} 2 \mathrm{Al}$ surfactant (Dow Chemical Company) was used as an etching solution, and the et.ching temperature was controlled at $80^{\circ} \mathrm{C}$.

\section{Results and Discussion}

With the etching conditions used in these experiments, an etching curve of polyester films obtained by the use of different etching times is presented in Figure 3.5. Pore sizes in this figure were determined from He gas permeability tests and diffusivity measurements of n-octane in ethyl acetate. In the pore diameter calculations done with the experimental results of diffusivity measurements, hindrance effects were reflected by the use of the equation proposed by Brenner and Gaydos (40). Details of this procedure are described in the next section (membrane characterization). As shown in Figure 3.5 , no permeation of n-octane was observed in the diffusion experiments with 


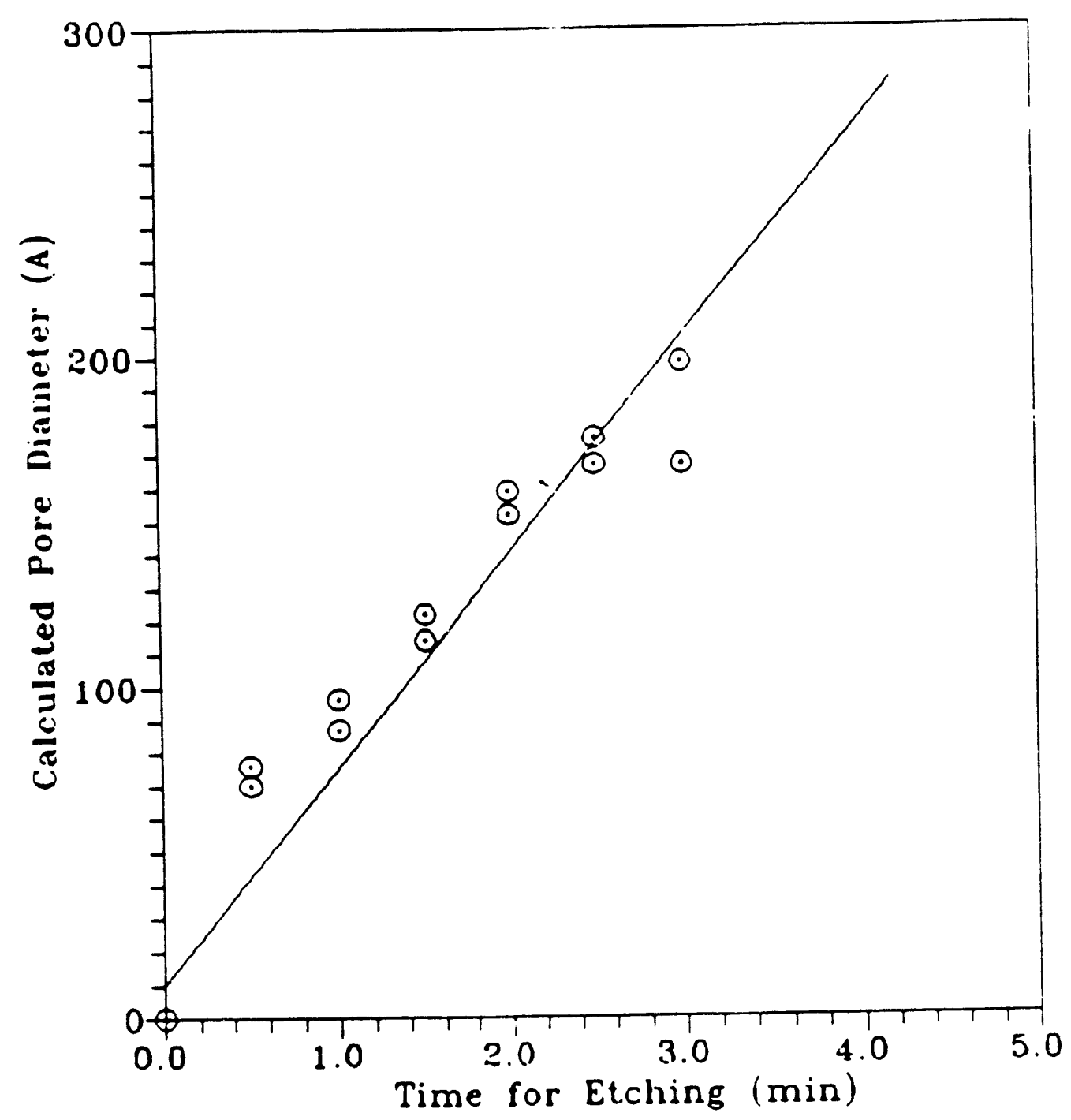

Figure 3.5 Etching Curve of Polyester Films ( $1 \mathrm{~N} \mathrm{NaOH}$ sol'n, 0.02 wto Dowfax, (e $80^{\circ} \mathrm{C}$ ). 
unetched polyester membranes (no pores), and etching conditions to make polyester membranes having small pores suitable for the hinderea diffusion study were successfully found. The etching curve did not follow a straight line. The initial etching rate (less than $1.5 \mathrm{~min}$. ) appeared to be faster than the later etching rate. Qualitatively, this etching characteristic agreed with previous study (65) which reported that the etching rate of damaged core (about $50 \AA$ in diameter) was faster than that of undamaged area.

\section{Alumina Membranes}

Inorganic membranes have relative advantages over organic membranes due to their high temperature applicability, their durability in aggressive conditions (chemical, mechanical, and microbiological), and their high pressure applicability.

In Figure 3.6, a schematic drawing proposed by Keller et al.(74) for the structure of the alumina layer formed by the anodizing technique is presented. As shown in this figure, a porous layer formed on the aluminum surface by anodizing the surface in an acid electrolyte has a unique structure composed of closed-packed, hexagonal-shaped oxide cells containing micropores in the center of each cell. This structure of a porous oxide layer gives a unique characteristic membrane having a uniform pore size distribution as seen in track-etched polymer membranes. Several research groups $(71,73-75)$ reported a method of 


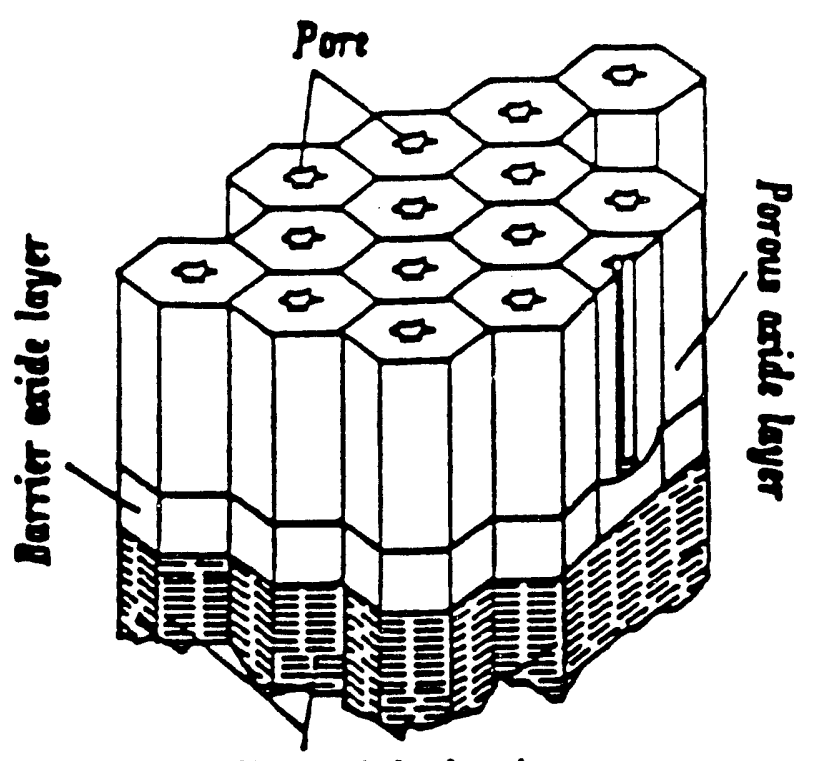

Onreatad chaminam

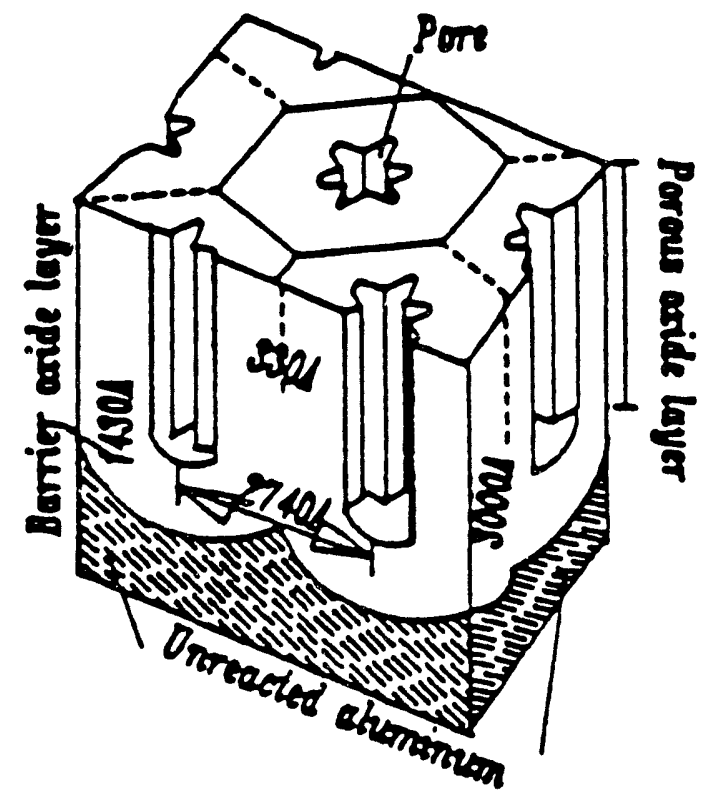

Figure 3.6 Model for Structure of Porous Aluminum oxide Layer (75). 
preparing an anodic alumina membrane and the results of its application in seperation research. Many studies have also reported characteristics and formation mechanisms of anodic alumina membranes (110-114).

An experimental study was conducted to prepare the anodic oxidation alumina membrane and an applicability of the prepared alumina membrane to the present study was investigated.

\section{Experimental}

pretreatment. The aluminum foil used in this study was 99.999 grade pure aluminum (Alfa Product, Morton Thiokol, Inc.) having $0.25 \mathrm{~mm}$ thickness. Circular specimens of $50 \mathrm{~mm}$ in diameter were cut from the parent aluminum foil and washed in acetone, followed by methanol. After cleaning, the aluminum specimen was placed on a press in order to make the surface of specimen flat. Clean, spent silicon wafer plates having no surface defects were attached to the bottom and top sides of the press to keep the aluminum specimen from acquiring any surface defects during the pressing process. Pressure applied on the surface of the aluminum foil was ca. $1 \times 10^{8} \mathrm{~Pa}$. After pressing, the aluminum specimen was annealed for about an hour in the furnace at $350{ }^{\circ} \mathrm{C}$ to remove the stress produced during the pressing process.

The annealed aluminum was further treated by a chemical polishing to remove the irregularities of the surface, 
because on a microscopic scale the surface still had complex and small irregularities. The chemical etching solution was made of 75 parts sulfuric acid, 20 parts phosphoric acid and 5 parts nitric acid. The aluminum specimen was treated with this etching solution for 10 seconds at $170^{\circ} \mathrm{C}$. After polishing, the specimen was washed with distilled water and dipped in a chromic acid solution $\left(\mathrm{Cr}_{2} \mathrm{O}_{3}: 458, \mathrm{H}_{3} \mathrm{PO}_{4}: 3.58\right.$ in volume) to desmudge it, then washed with water and dried in air. Next, the periphery of the aluminum specimen was masked with Microshield ${ }^{T}$ Lacquer (Pyramid Plastic Inc.). In this masking process, the specimen was masked such that the unmasked area of the anodization side was greater than the unmasked area of the opposite side. This masking technique ensured uniform anodic oxidation across the area of unmasked aluminum surface of the anodization side, thus the specimen breakage was avoided during the etching treatments for the removal of non-reacted aluminun and the barrier layer.

Anodic oxidation. In Figure 3.7, a schematic drawing of the anodization cell used in the current study is presented. The pretreated aluminum specimen was placed between two o-rings and clamped so that it formed a barrier between two chambers of the anodization cell. Then, one chamber of the anodization cell was filled with electrolyte and the other side was filled with water. A 4 wt: oxalic acid was used as an electrolyte throughout the experiments. 


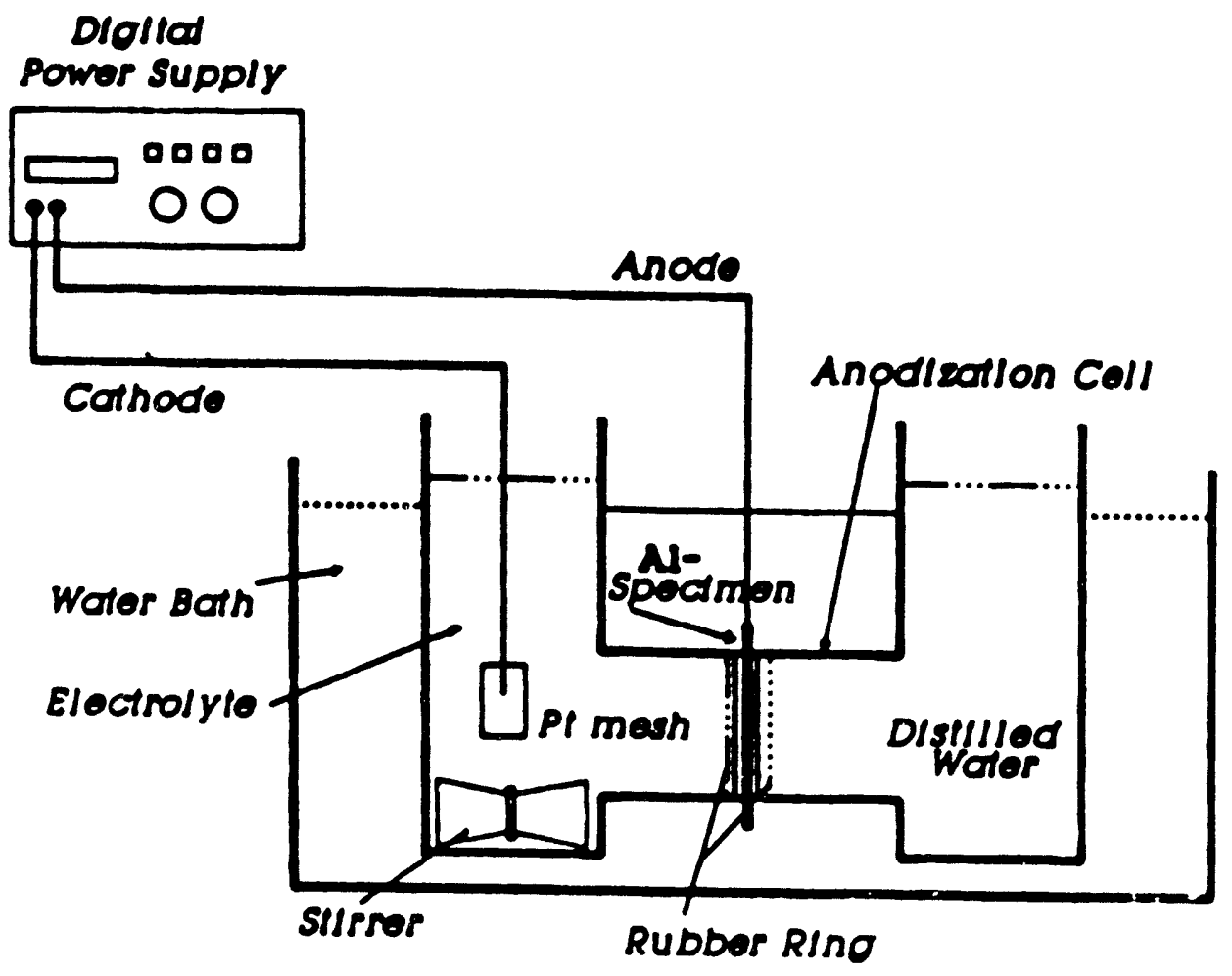

Figure 3.7 Diagram for the Anodization Cell 
water in the other side of electrolyte chamber was used mainly for cooling purposes since the anodic oxidation reaction was exothermic. Platinum wire mesh with a size of $4 \times 4 \mathrm{~cm}$ was used as a cathode and aluminun specimen was used as ananode. It was reported that acid electrolytes also dissolve the porous layer to some extent, especially at high temperatures, and widen the diameter of the pore mouth (71). To reduce this dissolution effect and to maintain the uniformity of pore sizes, an ice water bath was used to keep the anodization temperature low. During the anodization, the electrolyte was stirred with a star-head magnetic stirrer bar to maintain a constant anodization condition. The power supply was controlled by an Ephortec potentiometer (model \# 433-5000). Applied voltages and corresponding maximum currents were preset by the potentiometer. Current density plays an important role in the formation of a porous oxide layer, together with the anodization time. It was reported that the theoretical rate of formation of the barrier layer at a current density of $10 \mathrm{~mA} / \mathrm{cm}$ is $0.3 \mu \mathrm{m} / \mathrm{min}$ (5). When the preset voltage was applied to the anodization cell, very rapid anodization occurred until the current was limited by the power source. During this stage of anodization, a barrier layer was formed. As the barrier layer grew, the current at its maximum started to decrease. However, current flow increased again after a certain period, and reached a steady-state value. The formation of 
the porous layer could be monitored by observing this steady-state current.

In general, as the applied voltage was decreased, the current flow was also decreased and time required for steady-state conditions (i.e., porous layer growth conditions) was shortened. This implies that the thickness of the barrier layer is decreased by decreasing the applied voltage. After a certain anodization time, power was shut off and the chamber where the electrolyte was filled was emptied and thoroughly washed with distilled water.

Removal of unreacted aluminum and barrier layer. As a result of anodic oxidation reaction, two different forms of oxide layers (i.e., barrier layer and porous layer) were formed on the surface of the aluminum specimen. In order to make a porous membrane from this partially oxidized specimen, the barrier layer and the unreacted aluminum of the specimen were removed by the use of the etching technique.

First, to remove the unreacted aluminum layer, the unreacted side of the anodization cell was filled with an etching solution (20 wtz $\mathrm{HCl}$ plus $0.1 \mathrm{M} \mathrm{CuCl}_{2}$ ) and stirred with a star-shaped magnetic stirrer bar at room temperature. only the unmasked area was attacked by the etching solution. When the unreacted aluminum layer was almost removed, a transparent aluminum oxide layer was seen between two chambers of the anodization cell. 
Following the removal of the unreacted aluminum, water in the reacted side of the anodization cell was replaced with an acetate buffer solution $(\mathrm{pH}=6)$ and the other side of the anodization cell was thoroughly washed with water and filled with a 10 wt phosphoric acid solution to remove the barrier layer.

The removal of the barrier layer was monitored by setting a capillary flowmeter atop the reacted side of the anodization cell as shown in Figure 3.8. By using this arrangement, approximately 1 foot of water head was constantly applied to the reacted side of the cell. This constant applied pressure kept the phosphoric solution backflowing to the reacted side of the anodization cell so that the porous layer was protected from dissolution. Dissolution time was dependent on the thickness of the barrier layer. As the barrier layer disappeared, the buffer solution level in the capillary flowmeter began to decrease. The permeability of the resulting alumina membrane was measured by observing the rate of decrease of the buffer solution level in the capillary flowmeter. A Hoffman ${ }^{\text {TM }}$ tubing clamp was attached to the Tygon ${ }^{T M}$ tube connecting the capillary flowmeter and the water reservoir. By pressing the Tygon ${ }^{T M}$ tube with the Hoffman ${ }^{T M}$ clamp screw, constant buffer solution levels in the capillary flowmeter were easily recovered, and the flow rates of the buffer solution were measured several times to obtain the liquid 


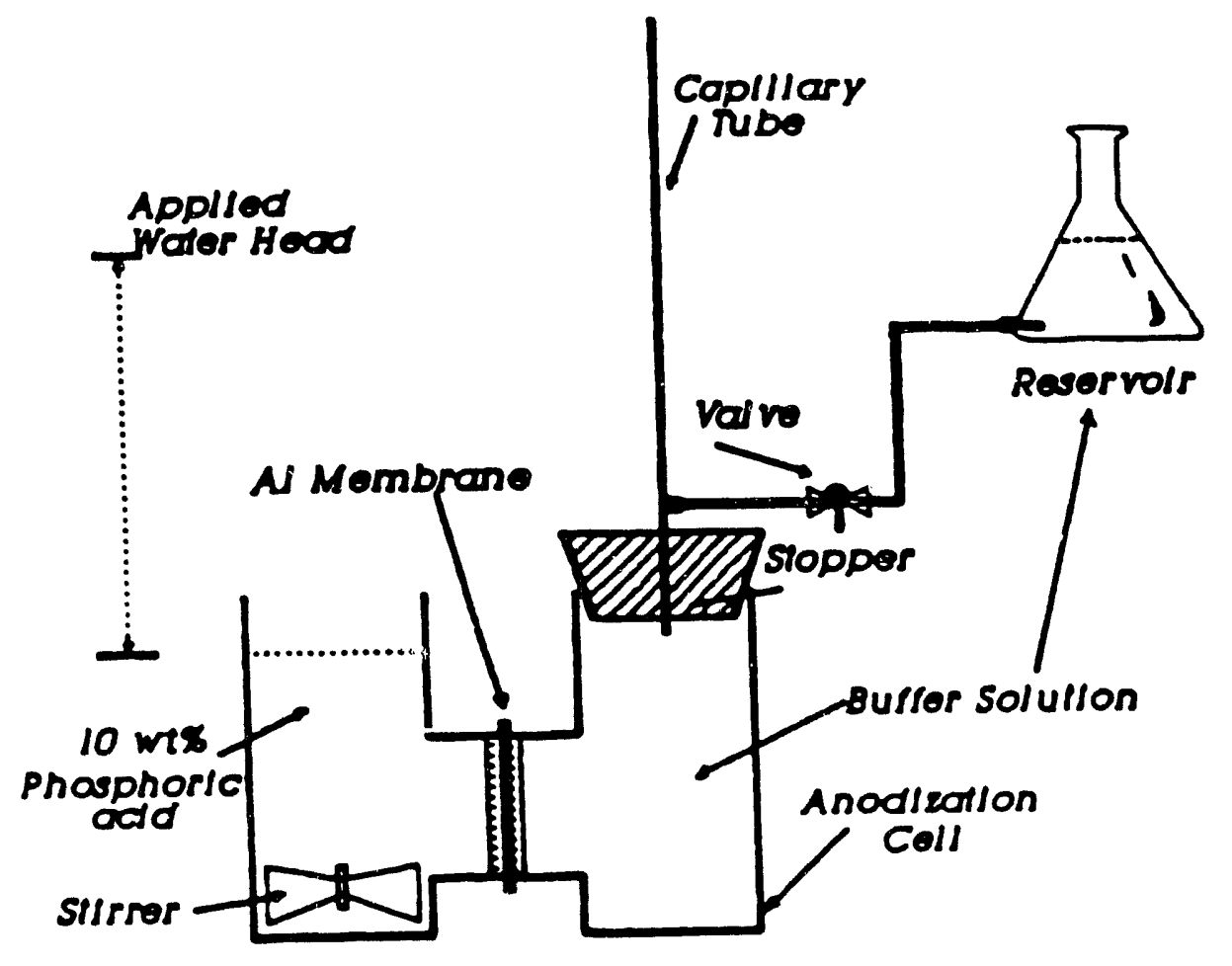

Figure 3.8 Apparatus for the Liquid Permeability Experiments in Anodic Oxidation Method 
permeability of the prepared alumina membrane. The average pore diameter of the alumina membrane was calculated from this liquid permeability data. The pore diameter was also measured with He gas permeability test.

\section{Results and Discussion}

In Table 3.1 , the average pore diameters and the thickness of alumina membranes made by the aforementioned procedures are summarized. More detailed studies of process parameters affecting the formation of alumina membranes using the same experimental apparatus and procedures were conducted by Chiou (76). According to this study, the minimum pore diameter of membranes obtained was $0.02 \mu \mathrm{m}$ with a thickness of $35 \mathrm{~mm}$. The author stated that membranes with a pore diameter smaller than $0.02 \mu \mathrm{m}$ were hard to prepare, since membrane thickness became thin and fragile at process conditions favorable for the formation of small-sized pore melubranes. However, the alumina membranes having this pore size $(0.02 \mu \mathrm{m})$ could not be used in the hindered diffusion study of coal asphaltene since the pore size was not small enough to give hindrance effects to the whole band of coal macromolecules. Attempts to prepare the alumina membranes with smaller pore size were not successful. Thus, the use of polyester membranes were thought to have more fotential at this time for the current study. However, the possibility of using an anodic oxidation technique with some 
Table 3.1

Average Pore Diameters (Prepared alumina membranes)

\begin{tabular}{|c|c|c|c|c|}
\hline $\begin{array}{l}\text { Membrane } \\
\text { number }\end{array}$ & $\begin{array}{l}\text { Anodization } \\
\text { condition" }\end{array}$ & $\begin{array}{l}\text { Thickness } \\
(\mu \mathrm{m})\end{array}$ & $\begin{array}{l}\text { Average } p \\
\text { Liq. Test }\end{array}$ & $\begin{array}{l}\text { dia. }(\mu \mathrm{m})^{d} \\
\text { Gas Test }\end{array}$ \\
\hline OC 18 & $\begin{array}{c}60 \mathrm{~V}, \\
4 \mathrm{hr} \\
\text { Anodization }\end{array}$ & 53 & 0.080 & 0.086 \\
\hline OC 20 & $\begin{array}{c}60 \mathrm{~V}, \\
4 \mathrm{hr} \\
\text { Anodization }\end{array}$ & 53 & 0.077 & 0.077 \\
\hline OC 23 & $\begin{array}{c}60 \mathrm{~V}, \\
8 \mathrm{hr} \\
\text { Anodization }\end{array}$ & 100 & 0.095 & 0.099 \\
\hline
\end{tabular}

a. Oxalic acid was lised as an electrolyte.

b. For the liqid permeability test, xylene was used.

c. For the gas permeability test, He gas was used.

d. Pore density used in these calculation: $\mathrm{N}=5.9 \times 10^{9}$ pores $/ \mathrm{cm}^{2}(71,75,76)$. 
modification to make alumina membranes with smaller pore sizes still remains. Recently, the applicabilities of anodic oxide alumina membranes in a diffusion study as well as in a reaction engineering study including catalysis have been reported by Nourbakhsh et al. (115-117). Because of the potential in the alumina membrane research, further studies on this subject are recommended as a future work.

\section{Membrane Characterization}

Track-etched polymer membranes can easily be defined by three parameters: average pore diameter, pore density and pore length. Information on these membrane characteristic parameters is required in order to calculate solute diffusivities from the diffusion experimental data as shown in equations 2.1 and 2.2 .

Several experimental techniques can be applied to obtain these characterization parameters. Details of characterization methods used in the present study, as well as the experimental results, are presented in this section.

\section{Pore Density}

In Figures $3.9,3.10,3.11$ and 3.12 , scanning electron microscope pictures of commercial polycarbonate membranes and polyester membranes (Nuclepore Corporation) are presented. As shown in these figures, the pore openings in these membranes are circular and the pores are identical. Pore densities car be found by counting the number of holes 


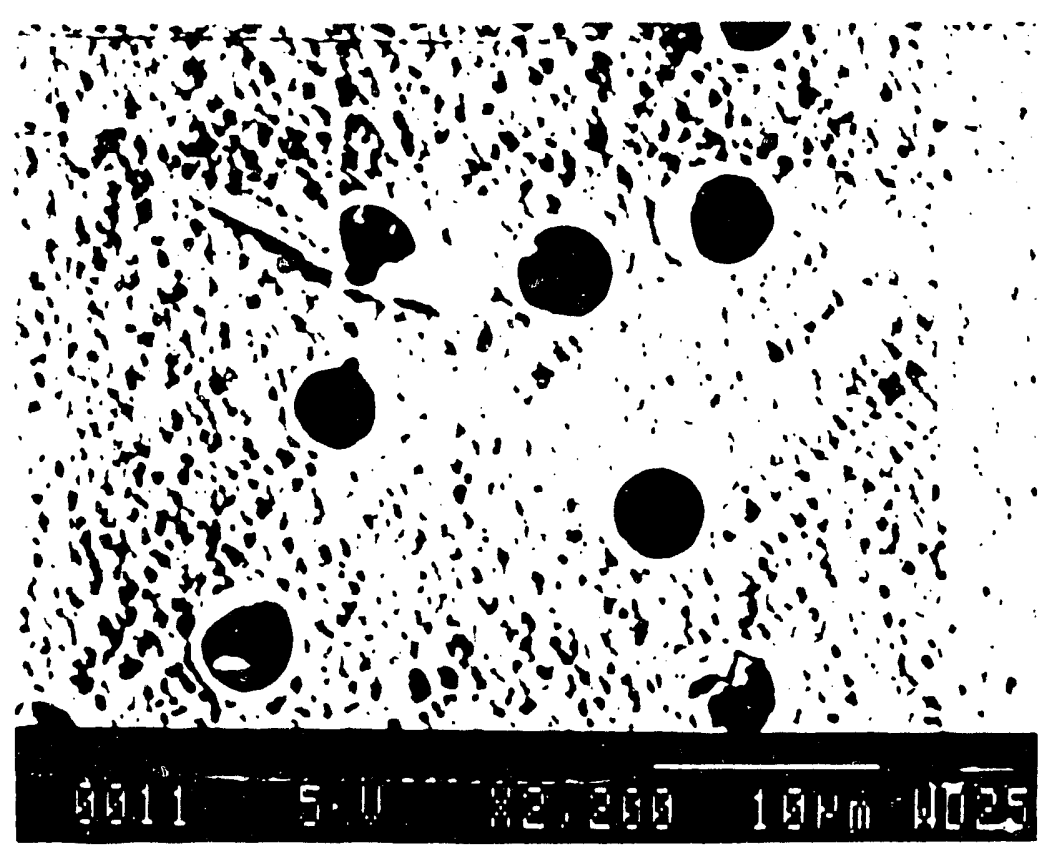

Figure 3.9 Scanning Electron Microscope Picture of Polycarbonate film. ( $5 \mathrm{\mu m}$ Nominal size)

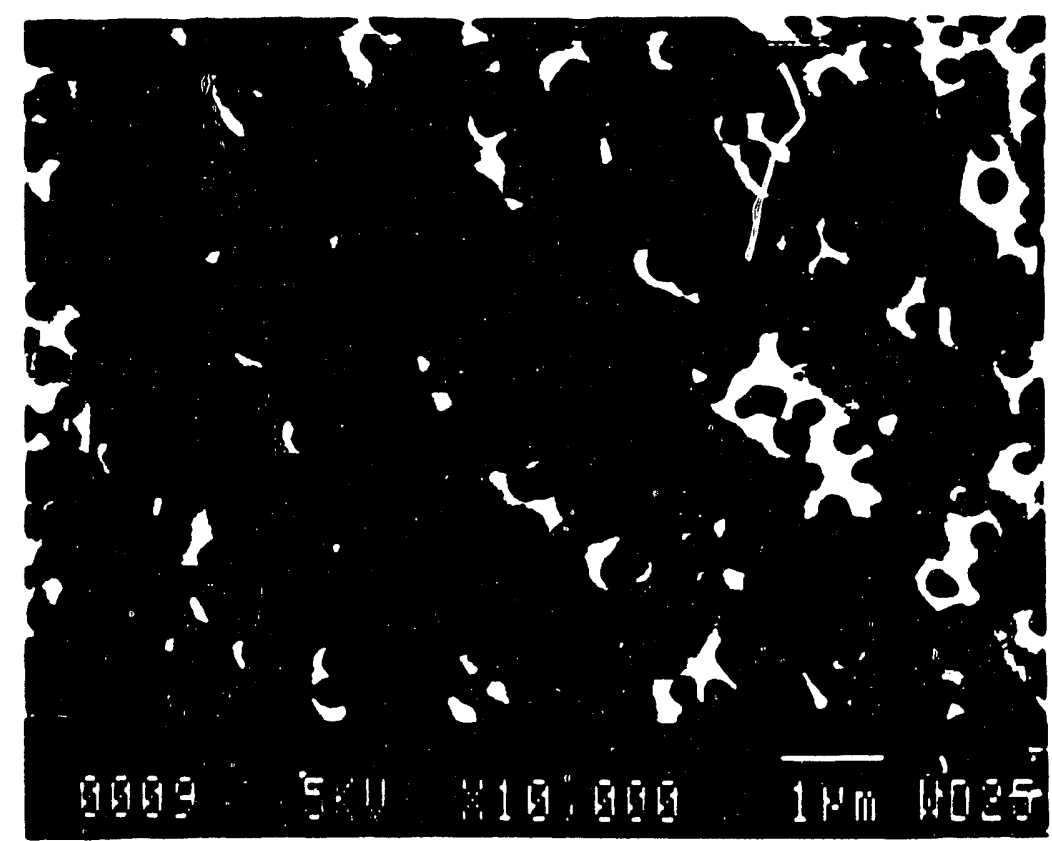

Figure 3.10 Scanning Electron Microscope Picture of Polycarbonate film $(0.2 \mu \mathrm{m}$ Nominal size) 


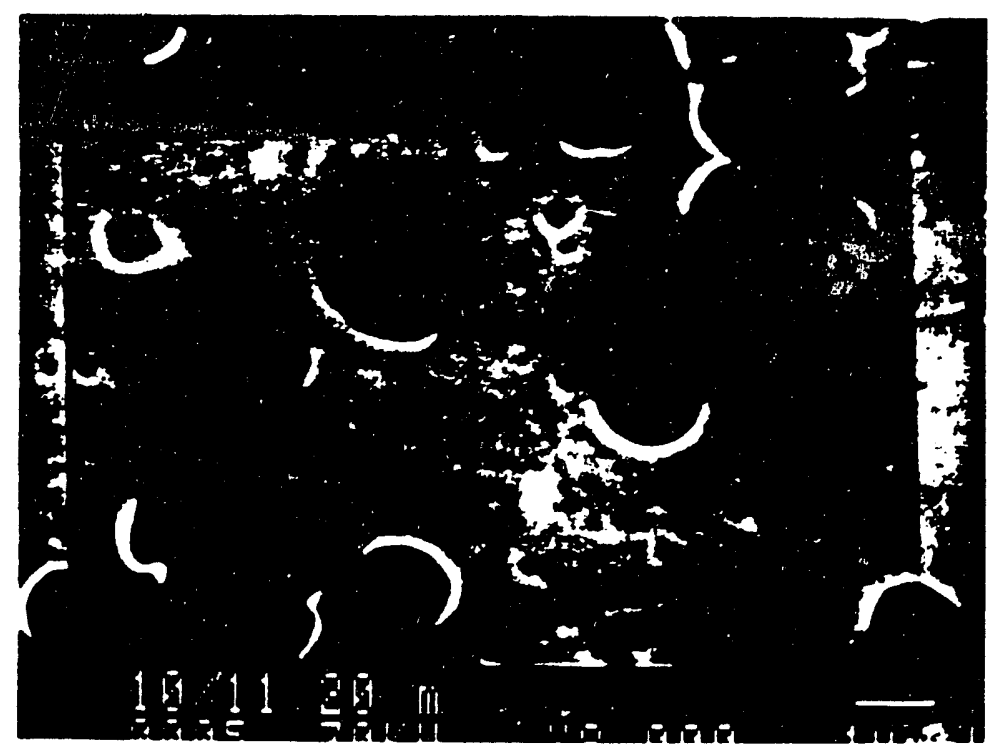

Figure 3.11 Scanning Electron Microscope Picture of Polyester Membrane ( $1 \mathrm{\mu m}$ )

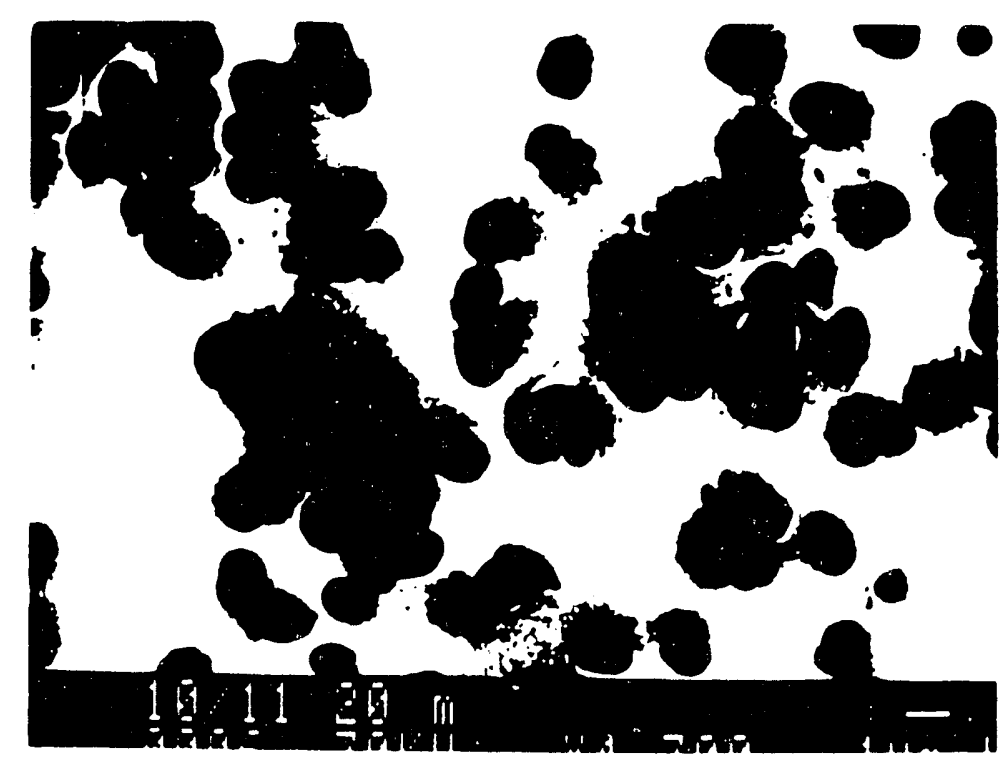

Figure 3.12 Scanning Electron Microscope Picture of Polyester Membrane $(1 \mathrm{\mu m})$ 
per unit area in these figures. Observed values of pore densities for these membranes $\left(3.27 \times 10^{8}\right.$ for $0.2 \mu \mathrm{m}$ polycarbonate membrane (Figure 3.10 ) and $2.13 \times 10^{7}$ for $1 \mu \mathrm{m}$ polyester memebrane (Figure 3.12)) were not much different from their rated value (within $\pm 10 \%$ ). Some studies using Nuclepore ${ }^{T M}$ track-etched membranes reported the same observations $(26,72)$. Also according to the manufacturer's product catalog (Nuclepore), mean pore density is held within the tolerance range \pm 15 \% of the rated pore density. However, Davidson et al. (105) observed that the measured pore densities of Nuclepore polycarbonate membranes using the scanning electron mocroscouy exceeded their norminal values by up to $50 \%$.

Practically, the technique using scanning electron microscopy to determine pore densities of membranes was not suitable for the current hindered diffusion study of coal macromolecules, because the resolution of SEM was not sufficient to see the small pores as low as $100 \AA$ and the technique was destructive of memebranes. Thus, In the current study, the use of pore density during the analysis of experimental data was avoided as much as possible by adopting the indirect measurement method which will be described in chapter IV. If the pore density was need in the calculation, the nominal pore densities were used. Instead, proper error analyses were conducted to investigate the effect of discrepancy between actual and nominal pore 
density. Details of these error analyses are reported in Appendix F.

\section{Pore Length}

The thickness of the polymer film from which tracketched membranes are made can be determined from the density of bulk polymer and the weight per unit area of film. Actually, the thickness of the polymer film used in the current study was very close to the rated values (the difference usually being less than $\pm 0.5 \%$ ). However, the pore length may differ slightly from the thickness of the membrane, since all the tracks formed during the irradiation process might not be aligned completely normal to the membrane surface (the maximum deviation was $29^{\circ}$ (as noted in Deen's work (26))).

Deen (26) calculated a correction factor, $6.8 \%$, which accounted for a difference between the average length of the pores and the membrane thickness based on the equal distribution of tracks within the maximum deviation angle. Direct measurements of this angle conducted by Cannel and Rondelez (47) with a transmission electron microscope on a Nuclepore ${ }^{T M}$ polycarbonate membrane, indicated that the angle was within $15^{\circ}$. The corresponding average pore length difference from the membrane thickness with this angle was calculated to be less than $1.2 \%$

Because of these variations in the angles observed, rated membrane thicknesses were used as pore lengths in the 
present study. Average errors caused by this simplication will be discussed in Appendix F.

Average Pore Size

Average pore sizes of membrane can be determined from direct measurement methods such as scanning electron microscopy and transmission electron microscopy or indirect measurement methods such as permeability tests with gases or Iiquids, or diffusivity measurement of a solute with known diffusion coefficient. The indirect measurement methods require information on pore density and pore length of a membrane. Experimental methods and corresponding results to measure the average pore sizes of membranes used in the current study are discussed in the following sections.

\section{Permeability test with liguid}

Flow rates of water (Optima grade, Fisher scientific) through Nuclepore ${ }^{T M}$ membranes were measured with a simple experimental apparatus shown in Figure 3.13. In this experiment, the flow rates of water were determined by a capillary flow meter or by weighing the water collected in a certain time interval. An applied head was adjusted to make the water flow in the poiseuille flow region, and not to exceed $14 \mathrm{~cm} \mathrm{H}_{2} \mathrm{O}$ because of irreversible deformation of polymer membrane. The diffusion cell was used directly to hold the membrane. Before the permeability test, water was prefiltered through a membrane having the same nominal pore 


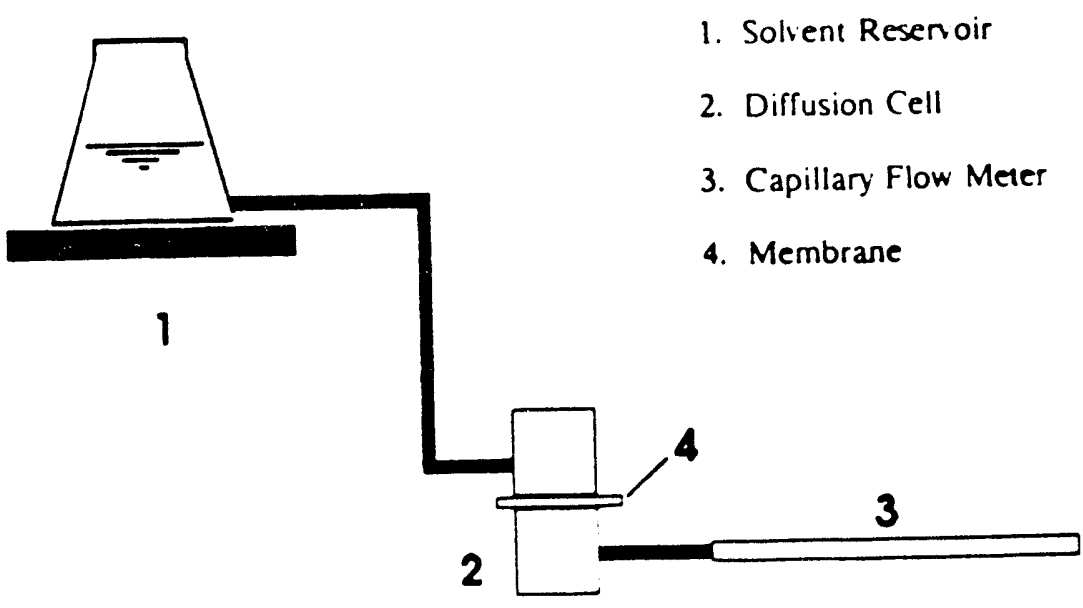

Figure 3.13 Liquid Permeability Test Apraratus 
size with one to be measured. A pressure filter adapter (Gelman Science, Inc.) was used to filter the water.

Experiments were conducted with six Nuclepore ${ }^{T M}$ membranes having different nominal sizes. With Poiseuille flow conditions, the average pore diameter can be calculated from the following equation:

$$
r_{0}=\left[\frac{8 \mu 1 Q}{n \pi A \Delta p g_{c}}\right]^{\frac{1}{4}}
$$

For other membrane parameters, pore densities and pore length, the rated values reported by the manufacturer were used in the calculation. Experimental results are presented in Table 3.2. Results show that the actual average pore diameters calculated from the liquid permeability tests are higher than their nominal rating of the Nuclepore ${ }^{i M}$ membrane. For example, for the membrane having $0.03 \mu \mathrm{m}$ nominal pore size, the actual size is about two times greater than the nominal rating. In previous studies using Nuclepore ${ }^{\text {M }}$ membranes, similar observations were reported that the actual measured diameters were always higher than the nominal rating diameters $(26-28)$.

\section{Permeability tests with gas}

The average pore diameter can also be calculated from the results of gas permeability tests. Flow rates of He and $\mathrm{N}_{2}$ gases through the membranes used in liquid permeability tests were measured with an apparatus shown in Figure 3.14 . 
Table 3.2

Actual Pore Diameters from Measurements (Nuclepore ${ }^{\text {TM }}$ Polycarbonate membranes)

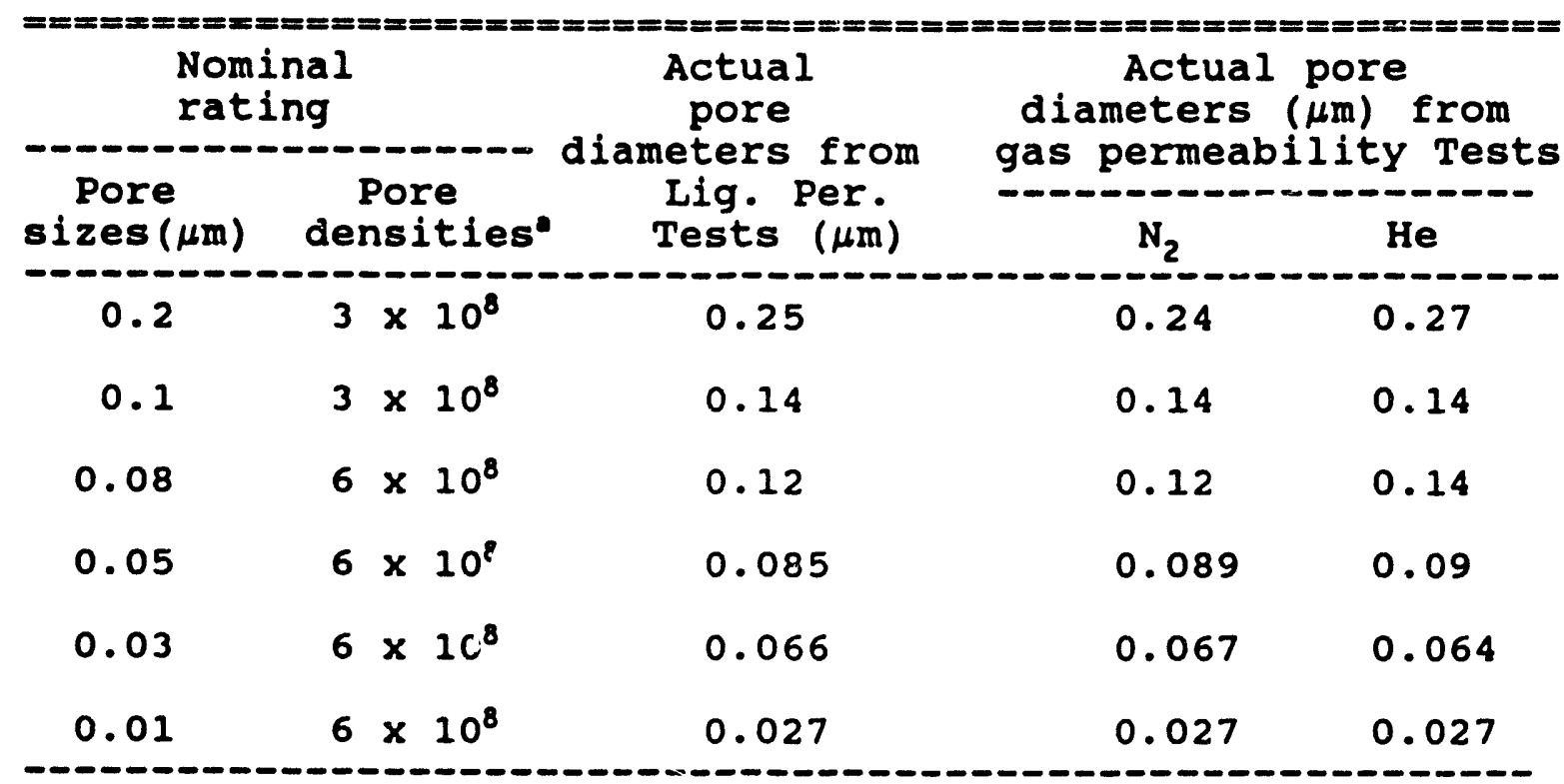

a. unit: number of pores $/ \mathrm{cm}^{2}$ 


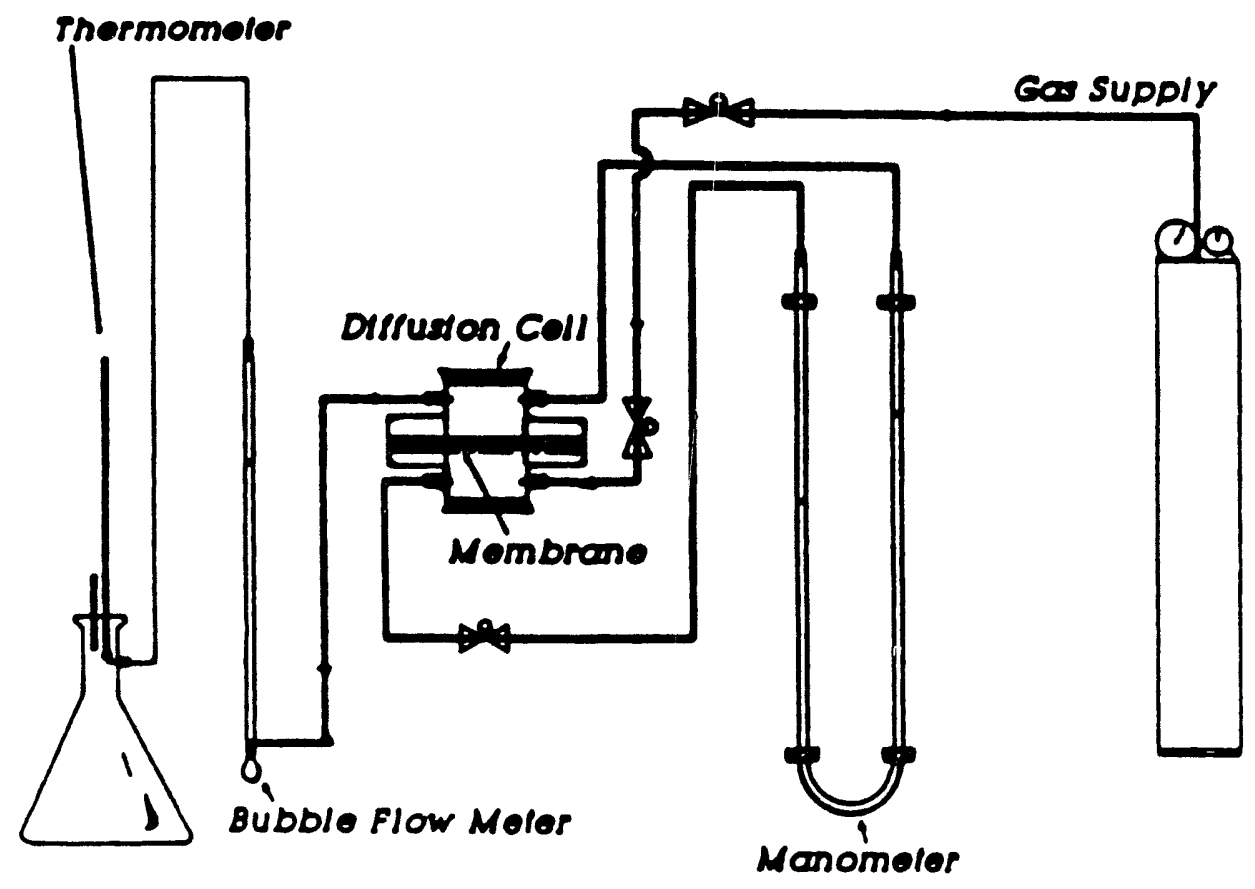

Figure 3.14 Gas Permeability Test Apparatus 
Again, the diffusion cell was used to mount the membrane. Two ports in each diffusion cell compartment were connected to a water manometer to measure the pressure drop at each gas flow condition. Gas flow was controlled by two needle valves in the inlet line. Other ports were used as the inlet and outlet line connections. A soap bubble flow meter was used to measure the gas flow rate. The transport of gas through a porous membrane consists of viscous flow, surface flow and Knudsen flow (15). Since the surfaces of membranes and gases used in these experiments were neutral, the effect of surface flow were neglected. Thus, the total gas molar flux can be expressed as:

Total $f \operatorname{lux}\left(Q_{g}\right)=K n u d s e n f l u x\left(Q_{k}\right)+\operatorname{Viscous} f \operatorname{lux}\left(Q_{v}\right) \quad(3.2)$ where,

$$
\begin{aligned}
& Q_{k}=\frac{4}{3} \frac{\pi r_{0}^{3} n \Delta p \sqrt{2 / \pi R_{1} T M}}{1} \\
& Q_{v}=\frac{\pi r_{0}^{4}\left(P_{1}^{2}-P_{2}^{2}\right) n}{16 R_{1} T I \mu}
\end{aligned}
$$

Using the above equations, the pore radii were calculated from volumetric gas flow rates and corresponding pressure drops. In applying Equation 3.2, when the pore diameter of the membrane approached the mean free path of the molecule (Knudsen flow region), the viscous flux term became insignificant. Again, the pressure drops were not to exceed $14 \mathrm{~cm} \mathrm{H}_{2} \mathrm{O}$ for the same reason as in the liquid permeability tests. The results are also summarized in 
Table 3.2. Results show that calculated pore diameters are in agreement with the results from the liquid permeability tests. The closeness of the calculated diameters from the two different sets of experiments demonstrated that all assumptions made in the calculation, i.e., that there was no surface flow and that the rated values closely represented other membrane properties, were correct.

\section{Pore size determination from diffusion experiments}

As noted in the previous section, there were certain application limits in the liquid and gas permeability test to determine the average pore sizes of membranes, because of the low fluxes of gases and liquids at the limits in applied pressure for snall-sized pore membranes. It was observed that the measurable pore diameter limits with these permeability tests were about $0.015 \mu \mathrm{m}$. Thus, for the membranes having diameters less than $0.015 \mu \mathrm{m}$, pore diameters were determined from diffusion experiments. In this diffusion experiment, the diffusion rates of solute with known diffusivity were measured, and the pore radius was determined from following equation using the experimental data for $R_{T}$ :

$$
\frac{1 / n \pi r_{0}^{2}}{D_{\infty}\left[1+\left(\frac{9}{8}\right)\left(\frac{a}{r_{0}}\right) \ln \left(\frac{a}{I_{0}}\right)-1.54\left(\frac{a}{I_{0}}\right)\right]}=R_{T}
$$

where ' $a$ ' equals the radius of solute, and $D_{\infty}$ is the bulk diffusivity of solute used as a standard. This equation was 
81

derived from Equation 2.2 after ignoring the boundary layer resistance and the entrance and exit effect, followed by applying Equation 2.20 to reflect the hindrance effect in small-sized pore membranes. For the radius of solute, ' $a$ ', Stokes-Einstein radius was used, since the hindrance factor derived from Equation 2.20 was developed for the sphere model. To obtain the pore radius from a total transfer resistance $\left(R_{T}\right)$ using Equation 3.5 , a root finding method was used. In Table 3.3, a comparison table of pore sizes determined from diffusion experiments and gas permeability tests is presented. As shown in this table, both methods provide a fairly good agreement between the values measured for the pore diameters. 
Table 3.3

Average Pore Diameters Determined

from Diffusion Experiments

(Prepared polyester membranes)

\begin{tabular}{|c|c|c|c|c|}
\hline Membrane & $\begin{array}{c}\text { Etching } \\
\text { Conditions }\end{array}$ & $\begin{array}{c}\text { Cal. } \\
\text { from He } \\
\text { Permeability } \\
\text { Test (A) }\end{array}$ & $\begin{array}{l}\text { ore Diameter } \\
\text { C }_{8} \text { EA } \\
\text { Diffusion" } \\
\text { (A) }\end{array}$ & $\begin{array}{c}C_{8} / C_{10} \\
\text { Diffusion } \\
\text { (A) }\end{array}$ \\
\hline PE44001 & $\begin{array}{l}4 \mathrm{~N} 40^{\circ} \mathrm{C} \\
15 \mathrm{~min} .\end{array}$ & 255 & 274 & 258 \\
\hline PE4 4002 & $\begin{array}{l}4 \mathrm{~N} 40^{\circ} \mathrm{C} \\
15 \mathrm{~min} .\end{array}$ & 216 & 216 & 212 \\
\hline
\end{tabular}

a. The bulk diffusivity of $n$-octane $\left(C_{8}\right)$ in ethyl acetate (EA) used in this calculation: $2.45 \times 10^{-5} \mathrm{~cm}^{2} / \mathrm{sec}$., (Chapter VI)

b. The bulk diffusivity of n-octane in $n$-decane $\left(C_{10}\right)$ used in this calculation: $1.60 \times 10^{-5} \mathrm{~cm}^{2} / \mathrm{sec}$, (Chapter VI) 


\section{APPARATUS AND PROCEDURES FOR DIFFUSION EXPERIMENTS}

The experimental apparatus and procedures used in the diffusion experiments for the current research are described in detail below.

\section{Apparatus for Diffusion Experiments}

A diagram of the diffusion cell used in the present stuay is shown in Figure 4.1. The cell consisted of two equal compartments separated by a membrane and had two inlet and outlet ports in each compartment. The cell itself was similar to the design used by Baltus (121), except for its injection assembly and stirrer assembly. The body of the diffusion cell was made of a Pyrex ${ }^{\mathrm{TM}}$ glass flat ground joint, with a $32 \mathrm{~mm}$ outside diameter (O.D.), and Pyrex ${ }^{\mathrm{TM}}$ glass short-length tapered-ground joint with a $32 \mathrm{~mm}$ O.D. A Teflon ${ }^{\text {TM }}$ hollow stopper was fitted into the ground joint section at the end of each compartment. Luer outer joints with a $4 \mathrm{~mm}$ inside diameter (I.D.) were used as inlet-outlet ports.

The stirrer in each compartment was made from one solid piece of Teflon TM. A Teflon TM rod was machined such that the thin, circular-disk shaped top section was connected to the thick, cylindrical shaped bottom section by a thin shaft. 


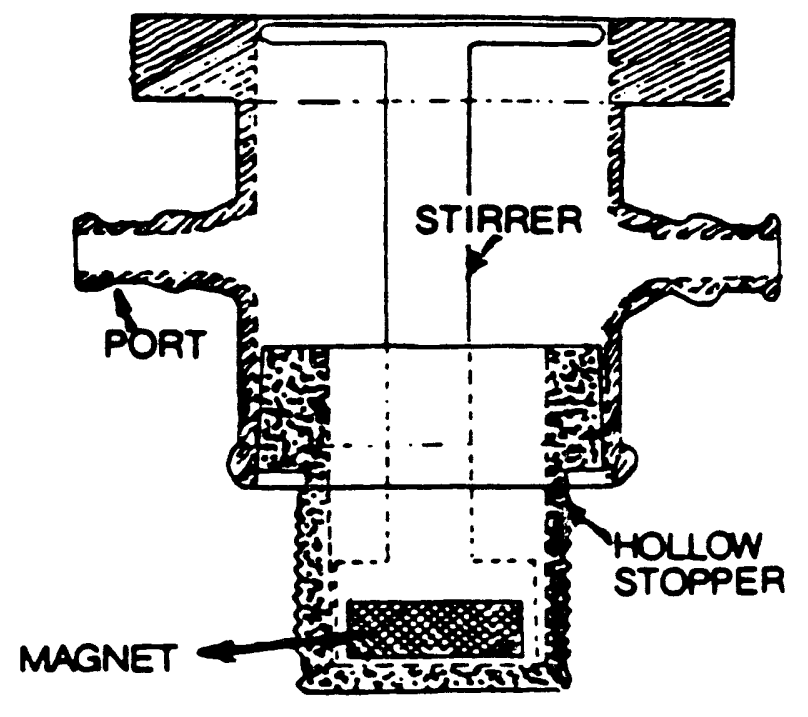

Figure 4.1. Schematic Drawing of Half Diffusion Cell. 
The thin disk (top section) had six equally spaced holes, each $6 \mathrm{~mm}$ in diameter, to facilitate the bulk movement of fluid within the compartment. The magnet, which was acted upon by an external magnetic force, was located inside the cylindrical shaped bottom section of the stirrer. The shape of stirrer is illustrated in Figure 4.2 .

The length of the stirrer was about $2 \mathrm{~mm}$ less than the overall height of one diffusion cell compartment measured from the inside bottom of the hollow stopper. The diameter of the top section was slightly less than the inside diameter of the diffusion cell and the diameter of the bottom section was also slightly less than the inside diameter of the hollow stopper. On the bottom compartment, the stirrer was seated within the hollow of the stopper and rotated by the external magnetic force. In the top compartment, the stirrer was supported by a stirrer support, installed within the hollow of the stopper. The support was also made of Teflon ${ }^{T M}$, had a doughnut shape, and was inserted around the stirrer shaft into the hollow of the stopper. The stirrers were driven by external magnets attached to the tips of shafts connected to the variable speed motor. Teflon ${ }^{\mathrm{TM}}$ valves with male Luer tips (Supelco, Inc.) were used to ensure a leak tight seal of the ports and to avoid pressure disturbances which occured when the diffusion cell was filled with a solvent or a stock solution was injected into the diffusion cell. 


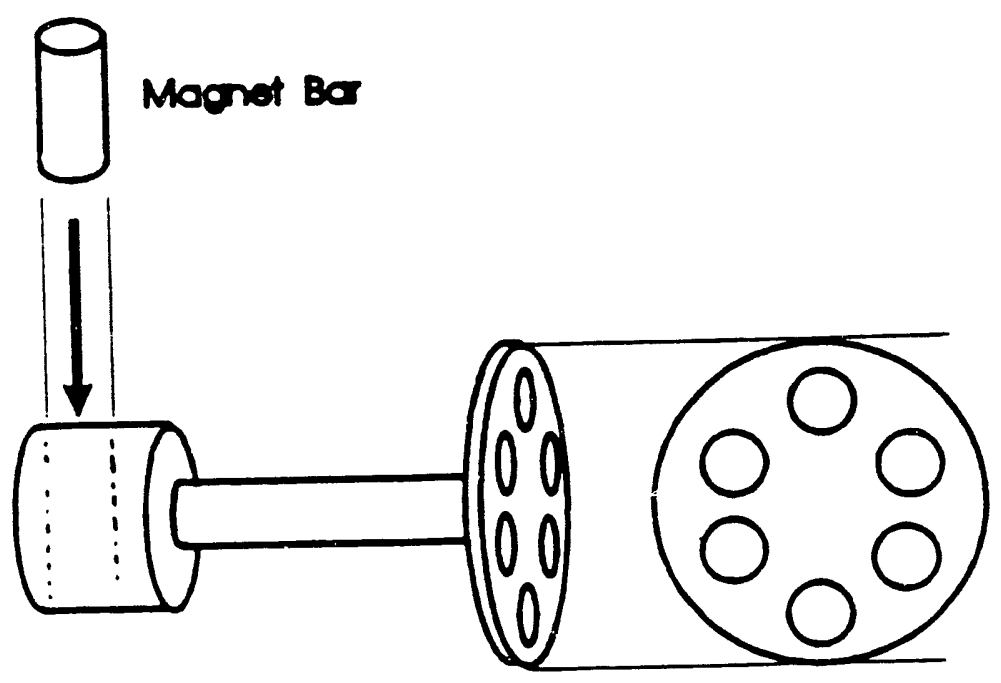

Figure 4.2 Schematic Drawing of stirrer 
Concentrated solutions (or stock solutions) were introduced to the diffusion cell by a gas tight Luer locktype syringe (1.0 ml capacity) with a Teflon ${ }^{\text {TM }}$ dead volume tube connected to the syringe needle. The end of dead volume tube was inserted into a Teflon ${ }^{\mathrm{TM}}$ male Luer plug having a tube size hole in the center. About $15 \mathrm{~mm}$ of the tube penetrated the plug and was bent into a horseshoe shape. The hole was sealed with chemical resistant epoxy (Abocoat 8501-1, Abatron, Inc.) after inserting the tube. This injection assembly design ensured the minimization of solute loss during the injection step.

Gaskets were made of blue Gylon ${ }^{\text {TM }}$ sheets and had dimensions of $32 \mathrm{~mm}$ I.D., $50 \mathrm{~mm}$ O.D. and $1.5 \mathrm{~mm}$ thickness. The membranes used in our experiment were supported by these gaskets. After placing the gaskets with a membrane between the faces of flat flanges of the cell compartments, the two cell compartments containing the stirrer assemblies were clamped tightly with external flanges made of aluminum. An aluminum rod $13 \mathrm{~mm}$ O.D. $\times 400 \mathrm{~mm}$ L. was attached to the aluminum flange to hold the diffusion cell assembly in place in a constant temperature water bath. A syringe containing stock solution was supported by a clamp attached to the aluminum rod.

To facilitate stirring in the cell, cyilndrical shaped rare earth $(\mathrm{Sm}, \mathrm{Co})$ magnets of $6 \mathrm{~mm}$ O.D. (Magnet Sales \& Manufacturing Co.) were used as the external magnetic 
source. One magnet was attached to the tip of the variable speed motor's shaft. The other magnet was fixed on the face of a rotor placed at the opposite side of the cell. The rotor shaft was connected to the $v$ iable speed motor by $a$ flexible power transmission wire. The rotation speed of the variable speed motor (Electro Craft Co.) was controlled from 0 to 1,000 rpm by servodyne speed controller (Cole-Parmer Co.) .

A rectangularly shaped 55-1iter aquarium was used as a constant temperature bath. The temperature of the bath was controlled by circulating water from a constant temperature circulating bath (Allied Co., Model 900). The circilating bath had a 3.7 liter reservoir capacity with $\pm 0.02{ }^{\circ} \mathrm{C}$ temperature control accuracy.

A schematic drawing of the overall system is provided in Figure 4.3. The variable speed motor is mounted atop the aquarium by using supports made of aluminum rods. Tygon $^{\text {TM }}$ tubes of $13 \mathrm{~mm}$ O.D. are used to connect the aquarium and the constant temperature circulating bath.

\section{Experimental Procedures}

All diffusion experiments were conducted in the batch experimental mode, i.e. so: tes were introduced to one compartment of the diffusion cell at time zero, arid concentrations of solute in both compartments of the diffusion cell were analyzed after allowing a certain diffusion time. 


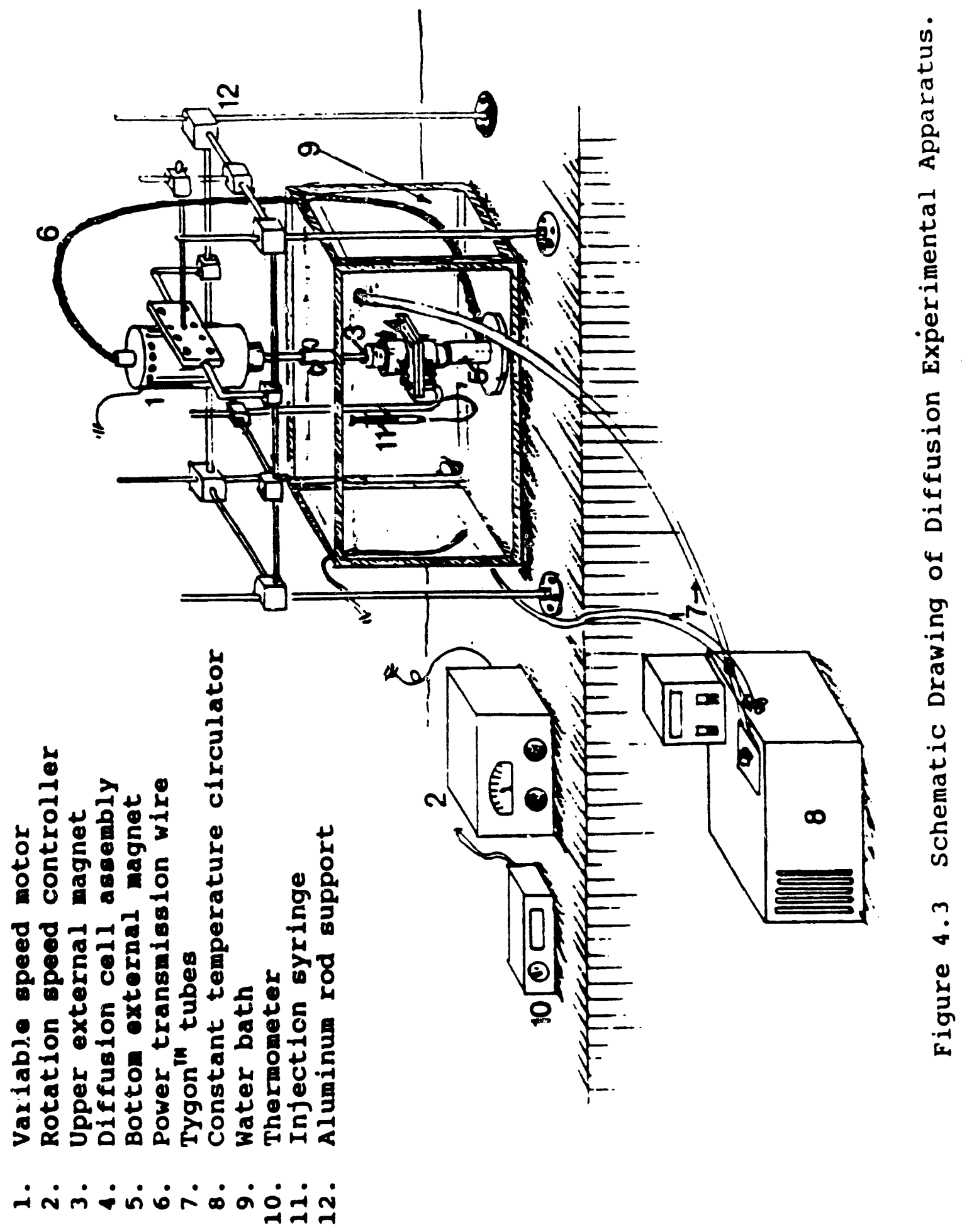


Before starting the diffusion experiment, all parts of the diffusion cell were cleaned with an acid cleaning solution (Nochromix (Godax Lab), in $98 \%$ sulfuric acid), rinsed thoroughly with distilled water, and dried in a vacuum oven. After drying, the diffusion cell unit was assembled.

Prior to the diffusion experiments, solvents were degassed in a sonicator for about 20 minutes to avoid possible bubble formation in the diffusion cell during the experiments.

Stock solutions (pure solute or concentrated solution injecting into the high concentration side of the diffusion cell at time zero) conditioned for diffusion experiments and analytical work (in terms of the concentration or the amount) were also prepared and degassed.

The diffusion cell was then filled with the degassed solvent by a syringe. During this process, the volumes of the cell compartments were also determined from the net weight of the solvent to fill each cell's compartment and the densities of the solvent. The net weights of the solvent were obtained by sequentially weighing the diffusion cell during the filling process. The net volume of one cell compartment was approximately $21 \mathrm{cc}$.

A small amount of stock solution (usually less than 0.5 cc) was taken by an injection syringe, and the injection assembly containing the stock solution was weighed. In the 
91

analysis of experimental results, the initial concentration of the high concentration side of diffusion cell was calculated from the net amount of stock solution injected into a diffusion cell. The amount was determined from the difference in the weight of the injection assembly before and after the injection. The male Luer plug connected to the tube of the injection syringe was fitted into the port of the diffusion cell after the cell was filled with solvent.

The entire diffusion assembly, including the injection syringe assembly, was then placed in a water bath, which maintained a preset diffusion temperature, for a certain time until thermal equilibrium was reached. Once the temperature was equilibrated, the diffusion cell assembly was taken out from the bath, and any pressure disturbance in the diffusion cell caused by volume changes of solvent was adjusted by opening the Teflon ${ }^{\text {TM }}$ valves inserted in the port of each compartment prior to the injection $n$ ' stock solution. The diffusion cell assembly was then put back into the water bath, and the stock solution was injected into the diffusion cell. During the injection, the Teflon ${ }^{\mathrm{TM}}$ valve fitted into the other side of injection port was left open to avoid pressure disturbances. Depending on the specific gravity of solute, the injection points of solute were changed in order to avoid any possible prediffusion of solute before the diffusion time was counted. Normally, the 
stock solutions having lighter specific gravities than solvent were injected into the upper compartment, and the ones having heavier specific gravities were injected into the bottom compartment. These injection times were recorded as starting times of the diffusion experiments.

Immediately after the injection of stock solution, stirring in the diffusion cell was also started. This stirring ensured the homogeneity in each compartment of the diffusion cell, and reduced the boundary layer resistance at both siles of the membrane surfaces. The stirring rates were mostly $500 \mathrm{rpm}$, which proved to be adequate for both purposes in the boundary layer study.

After a certain period of diffusion time, the entire diffusion cell assembly was removed from the water bath, and about $8 \mathrm{cc}$ of solution was sampled from each compartment of the diffusion cell by using syringes with 2-inch needles; the samples were stored in sample vials. In this sampling step, special precautions were required to avoid the convective flow, since any convective flow at this point would override the diffusional flux occurring during the experiment. Normally, this was done by manipulating the valves inserted to the ports of the diffusion cell and sampling sequentially.

Some materials used for the current diffusion study were sensitive to light and changed into ocher compounds by the photo-induced reactions. For experiments with these 
93

coumpounds (rubrene, coronene, etc.), the experimental apparatus was shielded from the light. In most cases, aluminum foil was used for this purpose.

Concentration analyses were performed with various techniques, depending on the diffusion system. These techniques included the differential refractometer, UVvisible spectrophotometer, gas chromatography and gel permeation chromatography. Details of these techniques will be discussed further in the following chapters. 


\section{DIFFUSIONAL BOUNDARY LAYER STUDIES}

An experimental study was conducted to characterize the diffusional boundary layer behavior in the diffusion cell used in the current study. From the results, an empirical equation which described the boundary layer was developed.

Based on this study, the effect of membrane properties on the boundary layer resistance was investigated to elucidate the conflicts regarding the significance of boundary layers in the hindered diffusion experiments found in the literature (27-30). Also, diffusion experiments with several solutes using alumina membranes were conducted to examine the boundary layer behavior with alumina membranes and the other alumina membrane properties.

\section{Experiments}

Two sets of diffusion experiments with solutes of known diffusivity (sucrose in water and n-octane in n-decane) were conducted to evaluate diffusional boundary layer

resistances. In this study, boundary layer resistances were calculated from Equation 5.1, with the total transfer resistances determined from experiments using different stirring rates, and with the membrane character zation information gained from the permeability tests: 


$$
R_{b}=\frac{R_{T}}{2}-\frac{1}{2 D_{\infty} n \pi I_{0}^{2}}-\frac{\pi I_{0}^{2} / 4}{D_{\infty} n \pi r_{0}^{2}}
$$

Next, an empirical equation relating the corresponding Sherwood numbers $\left(d / R_{b} D_{0}\right)$ to the Reynolds number $\left(\omega \cdot d^{2} \cdot \rho / \mu\right)$ (representing the stirring rates) was obtained from the results of boundary layer calculations.

Sucrose and Water system

The sucrose (reagent grade, specific rotation at $25^{\circ} \mathrm{C}$ : 66.3) and water (optima grade) were purchased from Fisher Scientific Co. Nuclepore ${ }^{\mathrm{TM}}$ polycarbonate track-etched membranes having $0.1 \mu \mathrm{m}$ nominal pore size were used throughout the experiments. The actual average pore size of these membranes, as determined from He gas permeability tests, was about $0.14 \mu \mathrm{m}$.

The concentrated sucrose solution $(0.584 \mathrm{M})$ was prepared first, then filtered through a polycarbonate membrane having $0.2 \mu \mathrm{m}$ nominal pore size. One batch experiment took about 60 minutes. At the end of each diffusion experiment, samples were taken from both compartments of the diffusion cell, and analyzed by a refractometer (Bausch \& Lomb, Co.) or a differential refractometer (Waters, model 400).

$$
\mathrm{N} \text {-octane and } \mathrm{N} \text {-decane system }
$$

$\mathrm{N}$-octane $(99 \%+)$ and $n$-decane (99\%) used in these experiments were purchased from Aldrich Chemical and Fisher 
Scientific, respectively. Two Nuclepore ${ }^{\mathrm{TM}}$ membranes, having $0.1 \mu \mathrm{m}$ nominal pore size (actual pore diameter $0.14 \mu \mathrm{m}$ ) and $0.05 \mu \mathrm{m}$ nominal pore size (actual pore diameter $0.09 \mu \mathrm{m}$ ), were used in these experiments. Actual pore diameter of each membrane was determined from He gas permeability tests. Diffusion experiments were conducted using the same procedures as in the sucrose diffusion experiments, except for the concentration analysis work. The concentration of n-octane was analyzed by gas chromatography, using the internal standard method. In this method, toluene was used as an internal standard to obtain a response factor. The response factor was determined from the known concentration ratio of n-octane to toluene in the calibrated solution, and from the peak area ratio of two components in the gas chromatogram. In Table 5.1, the operating conditions for the gas chromatograph are summarized. Samples from each experiment were taken in $8 \mathrm{cc}$ sample vials, and about $40 \mu \mathrm{l}$ of toluene was added to each sample. The unknown concentration of $n$-octane solution sample was determined from the peak area ratio between n-octane and toluene in a chromatogram, and from the response factor.

\section{Results and Discussion}

From the concentration changes of solutes, the total transfer resistance was calculated by the use of Equation 2.1. The boundary layer resistances were then evaluated with Equation 5.1, using the membrane resistance calculated 
Table 5.1

G. C. Conditions for Analysis

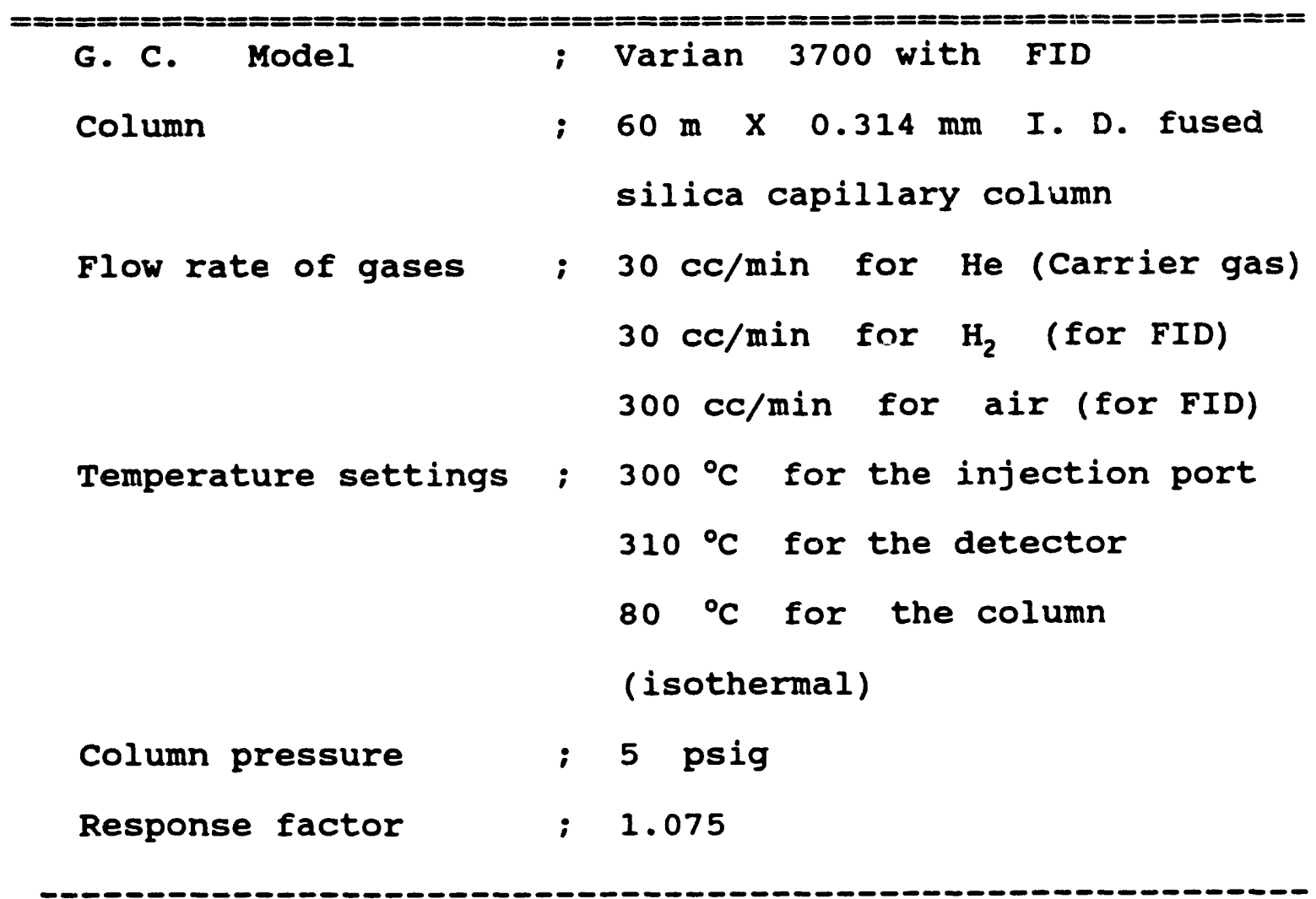


from membrane properties and the diffusion coefficients of solutes ( $D_{0}$ of sucrose in water, $5.26 \times 10^{-6} \mathrm{~cm}^{2} / \mathrm{sec}(77-79$ ) and $D_{0}$ of $n$-octane in $n$-decane used in this analysis, $1.62 \mathrm{x}$ $\left.10^{-5} \mathrm{~cm}^{2} / \mathrm{sec}\right)$. The experimental value of the diffusion coefficient of n-octane in n-clecane was not available in the literature; thus, it was estimated from the correlation equations found in the literature (the average of the values estinated from Lo's correlation ( 80 ) and Hayduk et al.'s correlation (81)). Details of these estimations are further discussed in Appendix $D$.

In Figure 5.1, a plot of $\operatorname{Ln}\left(\mathrm{N}_{\mathrm{sh}}\right)$ vs. $\operatorname{Ln}\left(\mathrm{N}_{\mathrm{Re}}\right)$ for ali sucrose diffusion experimental data is presented. The slope of this curve gave the exponential value of the $\mathrm{N}_{\mathrm{Re}}$ term, the intercept of this curve transferred to the correlation constants $(\alpha)$ in the empirical Equation 2.3. The correlation coefficient for this plot is 0.97 . The resulting empirical equation obtained from this procedure is as follows:

$\mathrm{N}_{\text {Sh }}=0.103 \mathrm{~N}_{\mathrm{Re}}^{0.66} \mathrm{~N}_{\text {Sc }}^{0.33}$

similar plots are presented for the results of n-octane diffusion experiments with the $0.1 \mu \mathrm{m}$ pore size membranes and $0.05 \mu \mathrm{m}$ pore membranes in Figures 5.2 and 5.3, respectively. In these plots, the correlation coefficients are both 0.98 . Using these plots, additional empirical equations were obtained as follows:

$$
\begin{array}{ll}
\mathrm{N}_{\text {Sh }}=0.057 \mathrm{~N}_{\mathrm{Re}}^{0.72} & \mathrm{~N}_{\mathrm{Sc}}^{0.33} \\
\mathrm{~N}_{\text {Sh }}=0.077 \mathrm{~N}_{\mathrm{Re}}^{0.69} & \mathrm{~N}_{\mathrm{Sc}}^{0.33}
\end{array}
$$




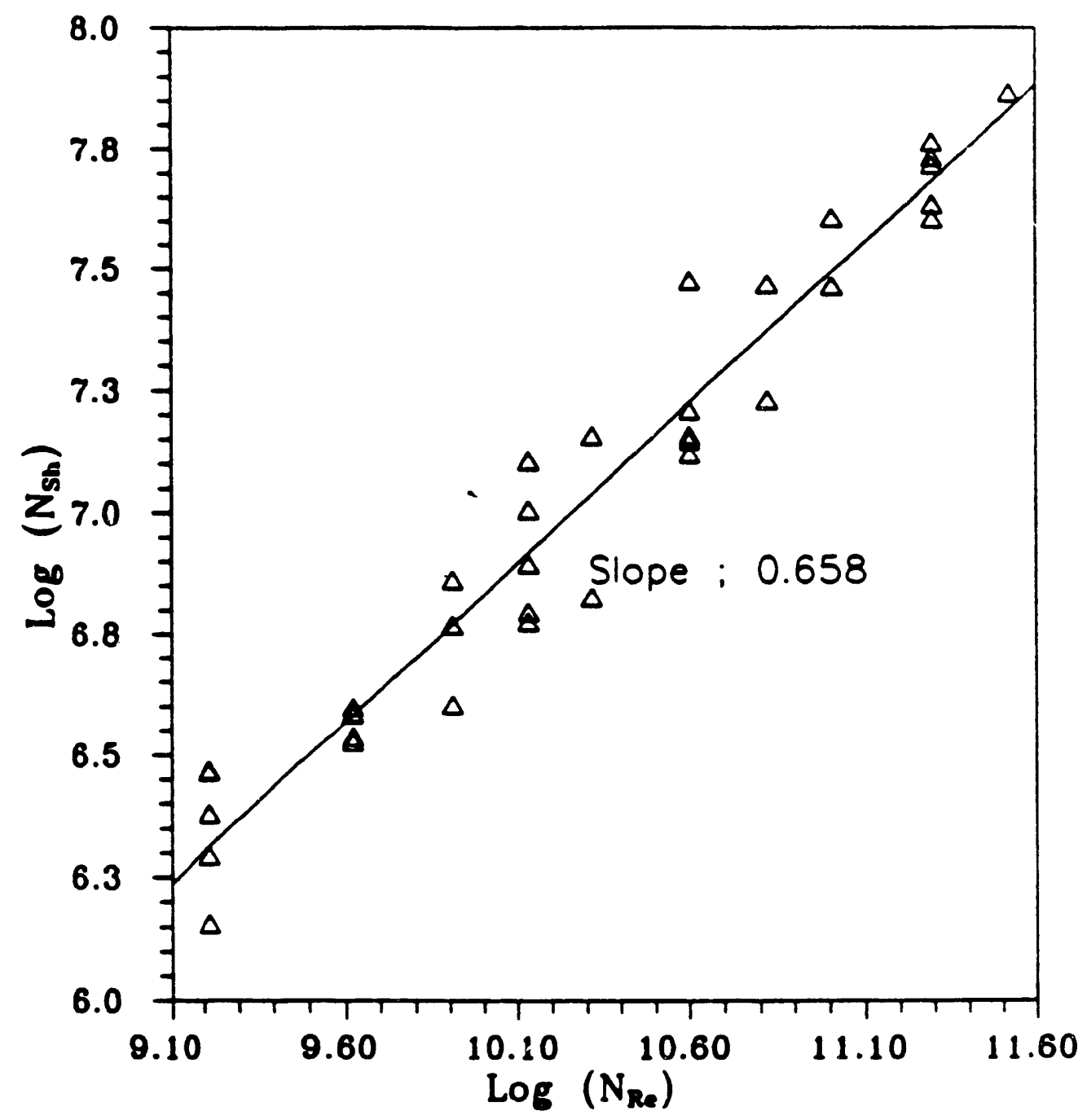

Figure 5.1. Nosh
(Sucrose/Water) 


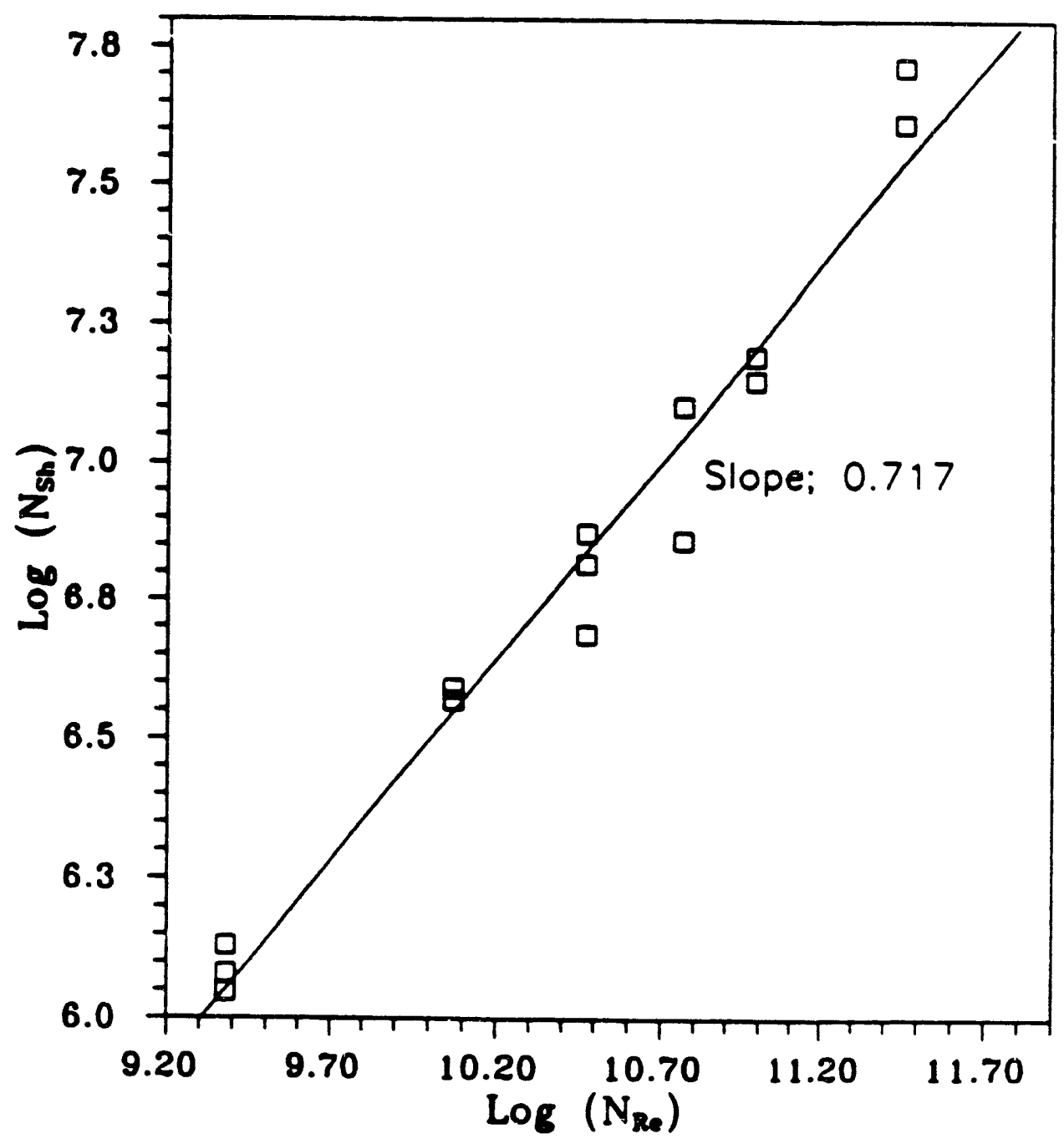

Figure 5.2. $\begin{aligned} & N_{\text {sh }} \text { vs. } N_{R e} \text { Correlation for } 0.1 \mu m \text { Membrane Data } \\ & \text { (N-octane } / N \text {-decane) }\end{aligned}$ 


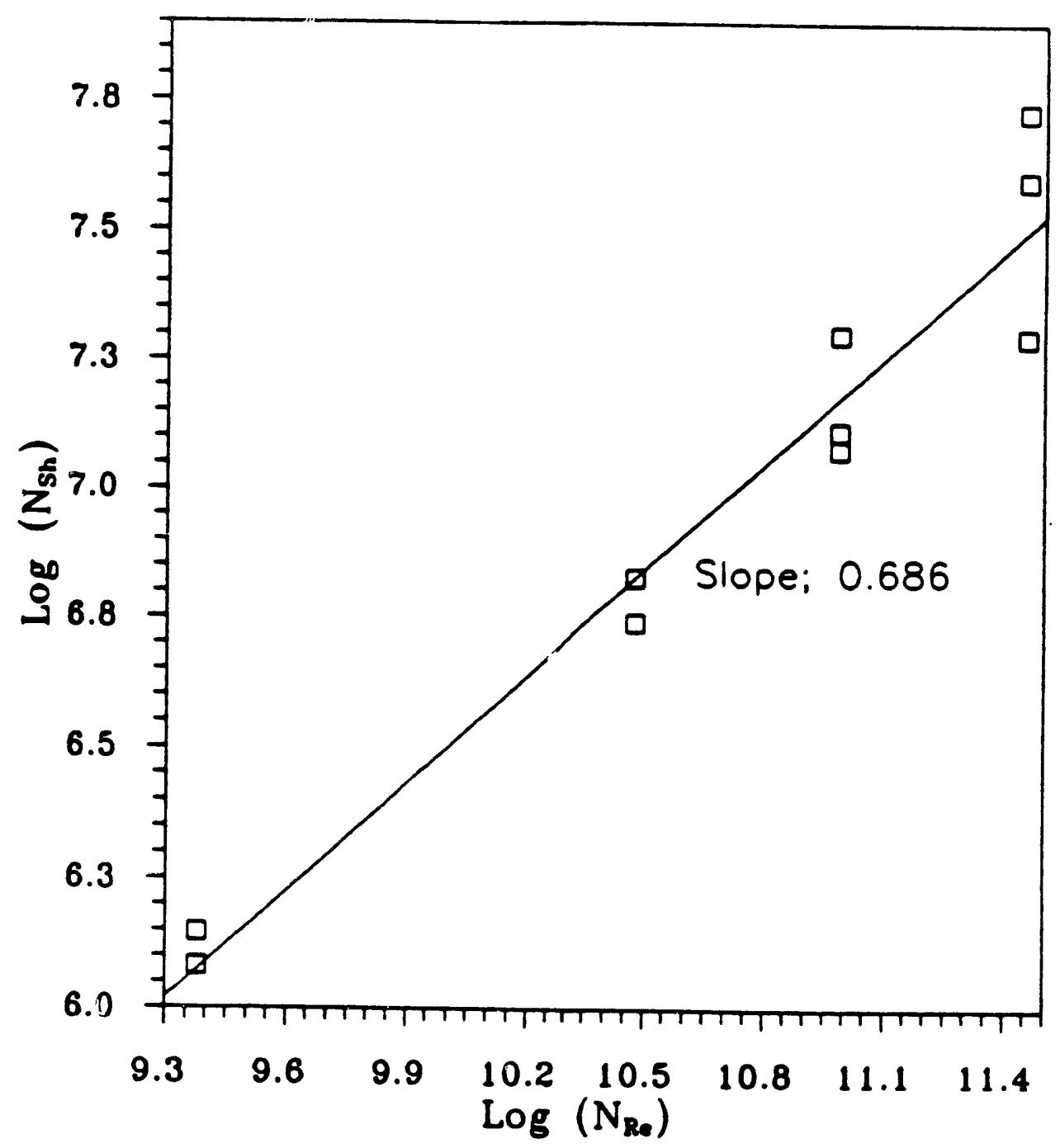

Figure 5.3. $\mathrm{N}_{\text {sh }}$ vs. $\mathrm{N}_{\text {Re }}$ Correlation for $0.05 \mu \mathrm{m}$ Membrane Data (N-octane / N-decane) 
The differences in the values of exponents and correlation constants in Equations $5.2,5.3$ and 5.4 are not important, since the calculated values of the sherwood Number $\left(\mathrm{N}_{\mathrm{sh}}\right)$, using the above three equations at certain Reynolds Numbers $\left(\mathrm{N}_{\mathrm{Re}}\right)$, were not significantly different. These observations can be seen more clearly in Figure 5.4, where all diffusion experimental data is presented in one figure. As shown in this figure (correlation coefficient: $0.98)$, all experimental data fit well into one correlation curve, regardless of the membranes adopted or the solutes used in the diffusion experiments. The empirical equation resulting from this plot is:

$$
\mathrm{N}_{\text {Sh }}=0.084 \mathrm{~N}_{\mathrm{Re}}^{0.63} \mathrm{~N}_{\mathrm{Sc}}^{0.33}
$$

Based on the preceding results, Equation 5.5 is the best empirical equation which describes the boundary layer behavior in the current diffusion experimental system. The numerical value of the exponent of the Reynolds number term in Equation 5.5 is close to that reported in a previous study (as seen in Table 2.1 (27)), where a similar type of stirrer (disk shaped) was used in the diffusion cell.

\section{Evaluation of Boundary Layer Resistances for Membranes Having Small Pore Sizes}

As noted in Chapter II, some previous studies $(27,28)$ in this area reported that the boundary layer resistance increased for low porosity membranes. This observation is important, since the boundary layer resistance is usually 


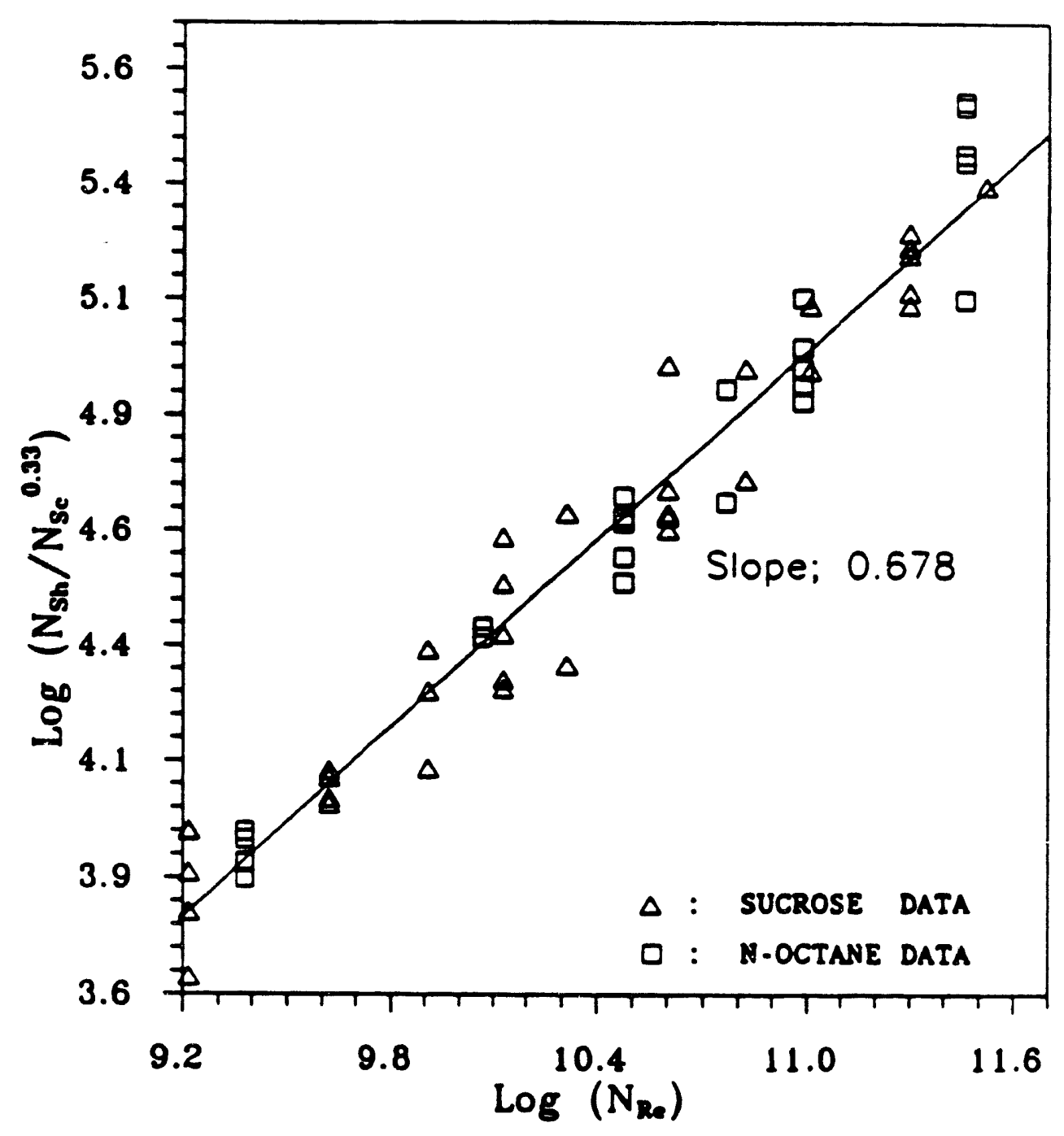

Figure 5.4. $N_{\text {sh }}$ vs. $N_{R e}$ Correlation for All Diffusion Experimental Data. 
considered small compared to the membrane resistance in most other hindered diffusion studies (29.30).

A study has been conducted to elucidate this matter. In the study, total transfer resistances obtained from experiments were compared with those estimated from the equations (the first term on right hand side of Equation 2.2 for the membrane resistance, Equation 2.20 for the hinderance factor correction and the Equation 5.5 for the boundary layer resistance).

In these experiments, Nuclepore ${ }^{\text {IM }}$ polycarbonate membranes having $0.01 \mu \mathrm{m}$ nominal pore sizes were used and a n-octane (solute)/n-decane(solvent) diffusion system was adopted. Membranes were characterized by the He gas permeability test prior to the diffusion experiments. Actual average pore diameter of the membranes used in this stuly was measured to be $0.029 \mu \mathrm{m}$. Details of experimental procedures and conditions were the same as the previous noctane diffusion experiments. The previous operating conditions were also used in the gas chromatography analysis.

In Table 5.2, the total transfer resistances obtained from experiments are summarized with the estimated total transfer resistances. As shown in the table, the total transfer resistances from the experimental results are not significantly different from the estimated values. The results imply that the boundary layer resistance is not 
Table 5.2.

Results of diffusion experiments with $0.01 \mu \mathrm{m}$ membranes (n-octane (solute) / n-decane (solvent)) e $25^{\circ} \mathrm{C}$

\begin{tabular}{|c|c|c|c|}
\hline \multirow{2}{*}{$\begin{array}{c}\text { Experiment } \\
\text { No. }\end{array}$} & \multirow{2}{*}{$\begin{array}{l}\text { stirring } \\
\text { rate } \\
\text { (rpm) }\end{array}$} & \multicolumn{2}{|c|}{$\begin{array}{l}\text { Total transfer } \\
\text { resistance }(\mathrm{sec} / \mathrm{cm})\end{array}$} \\
\hline & & $\begin{array}{l}\text { from } \\
\text { experiment }\end{array}$ & $\begin{array}{l}\text { from } \\
\text { estimationa,b }\end{array}$ \\
\hline OT001201 & 100 & 10,682 & 11,971 \\
\hline OT001203 & 500 & 10,193 & 11,244 \\
\hline OT001204 & 800 & 10,200 & 11,144 \\
\hline OT001502 & 100 & 10,104 & 10,553 \\
\hline OT001505 & 100 & 10,151 & 10,553 \\
\hline OT001504 & 300 & 9,798 & 10,158 \\
\hline OT001507 & 300 & 9,574 & 10,158 \\
\hline OT001501 & 500 & 8,539 & 9,836 \\
\hline OT001506 & 500 & 9,373 & 9,836 \\
\hline OT001503 & 800 & 8,441 & 9,738 \\
\hline
\end{tabular}

a. The estimated rotal transfer resistances are calculated from the first term on right hand side of Equation 2.2 for the membrane resistance and from Equation 5.5 for the boundary layer resistance.

b. It is important to note that the estimated resistances generally exceed the measured resistances showing that $R_{b}$ does not actually increase for low porosity membranes as stated by Bohrer $(27,28)$. 
changed as the membrane properties change. To examine this observation more closely, the boundary layer resistances calculated from the $0.1 \mu \mathrm{m}$ and $0.05 \mu \mathrm{m}$ membrane experiments are plotted against the stirring speeds using a Log-Log scale in Figure 5.5. The results of the $0.01 \mu \mathrm{m}$ membrane experiments are not presented in this figure, since the boundary layer resistance is so small in comparison to the membrane resistance that the calculation of this small number from the total resistance results in a significant error. In Figure 5.5, the solid line is the boundary layer resistance calculated from the empirical equation (Equation 5.5). As shown in Figure 5.5, the change of boundary layer resistance is only a function of the stirring speed in each set of experiments, which means that the boundary layer resistance does not depend on the membrane properties. This result is in accord with the theoretical considerations of Keller et. al (75).

On the basis of present observation, it was thought that the experimental results reported in Bohrer et al.'s work $(27,28)$ might be attributed to the adsorption of solute in the membrane pores, or to the plugging of pores by impurities during the diffusion experiments.

In conclusion, the boundary layer resistance is not affected by the membrar. properties, and can be estimated from the empirical Equation 5.5 developed in the current study. 


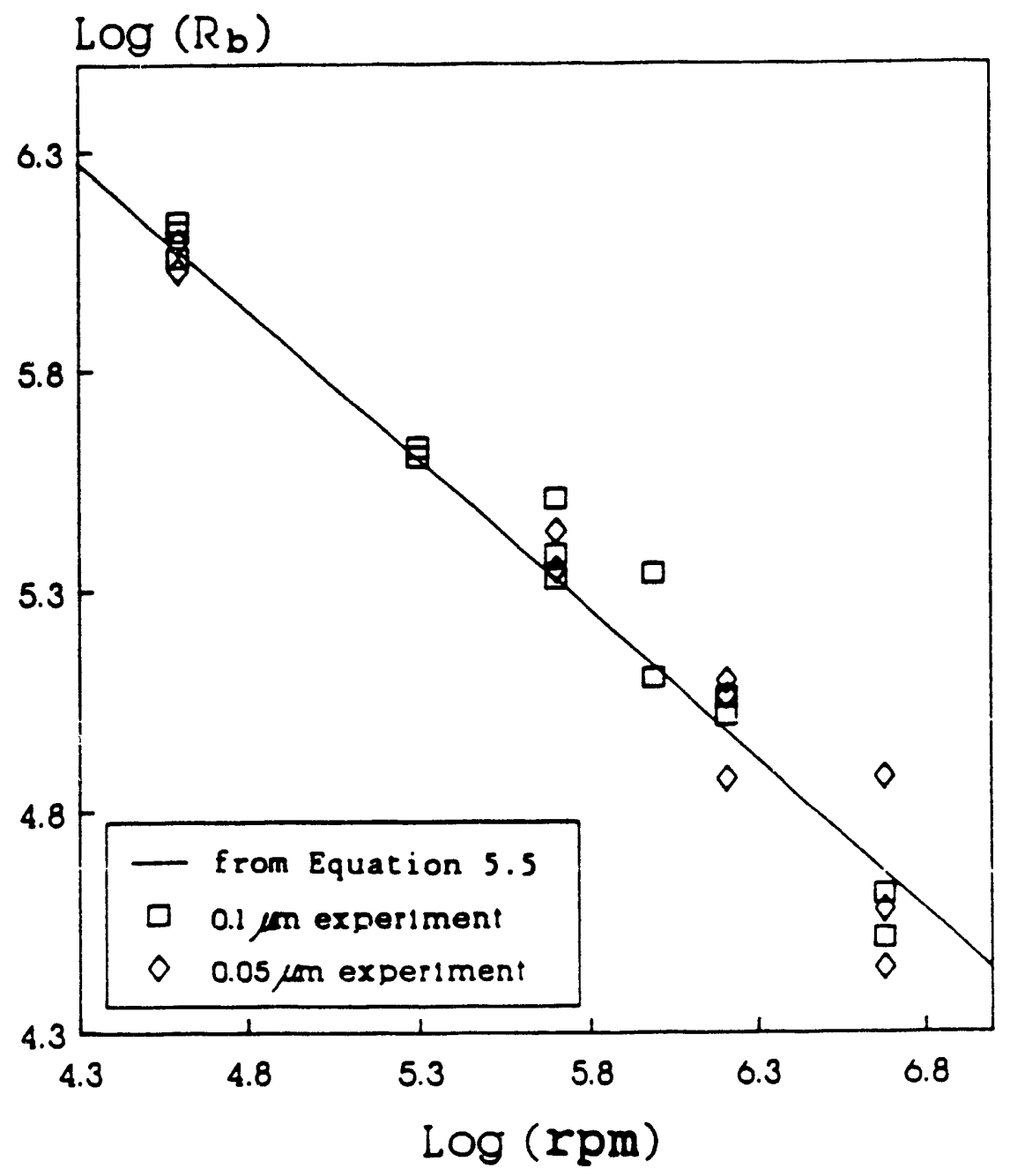

Figure 5.5. Boundary Layer Resistances from Experiments and Empirical Equation. 
Diffusion Experiments with Alumina Membranes

Based on the results of the koundary layer study, a diffusion study with commercially available alumina membranes was conducted to investigate the boundary layer behavior in diffusion experiments with alumina membranes and to characterize the membranes.

As previously noted in Chapter II, inorganic membranes have several advantages over organic membranes with regard to their applicability in catalysis and reaction engineering research due to their inertness.

Although several studies concerning alumina membranes have been performed in the past $(71,74,102)$, little has been reported about the results of liquid phase diffusion experiments using alumina membranes. A better understanding of the membrane properties and transport phenomena through the membrane are needed to utilize the alumina membranes in catalysis research.

In this study, sucrose/water diffusion experiments and n-octane/n-decane diffusion experiments were conducted with alumina membranes, so that results could easily be compared with the results from the diffusion experiments with tracketched polymer membranes.

Before the diffusion experiments, the liquid permeability tests and gas permeability tests were carried out to characterize the the alumina membrane. 
Experiments

Alumina membranes used in this study were obtained from Anotec Separation Inc (New York, NY). In Table 5.3, the major properties of alumina membranes are cited from the product catalog. All the experimental procedures and materials used as solutes and solvents were the same as those used in the diffusion experiments for the boundary layer study. Also, the same analytical techniques were used to analyze the concentrations of solutes (i.e., a differential refractometer was used in the sucrose/water diffusion experiments and a gas chromatography was used in the n-octane/n-decane diffusion experiments).

In the permeability tests using both liquid and gas to characterize the alumina membranes, water (optima grade, Fisher scientific. Co) was used in the liquid permeability test and He gas was used in the gas permaability test.

Results and Discussion

In Table 5.4, the results of permeability tests are summarized. Four different membranes from the same lot were tested. For each membrane, four different measurements of the gas permeability and liquid permeability were conducted. The thicknesses of membranes were measured with a micrometer. Pore densities were not measured, instead their values were taken from the product catalog. As shown in Table 5.4, calculated average pore diameters from both liquid permeability tests and gas 
110

Table 5.3

Properties of Alumina Membrane

\begin{tabular}{|c|c|c|c|c|}
\hline Brand name & ; & ANODISK 4 & 47 & \\
\hline Nominal pore size & ; & $0.2 \mu \mathrm{m}$ & & \\
\hline Pore density & ; & $3 \times 10$ & (pores) & $\left(\mathrm{cm}^{2}\right)$ \\
\hline Thickness & ; & $\mu \mathrm{m}$ & & \\
\hline
\end{tabular}

a. from product catalog of Anotec Separations (New York, NY) . 
Table 5.4

Results of the Permeability Tests

of Alumina Membranes.

\begin{tabular}{|c|c|c|c|}
\hline \multirow{2}{*}{$\begin{array}{c}\text { Membrane } \\
\#\end{array}$} & \multirow{2}{*}{$\begin{array}{l}\text { Thickness } \\
(\mu \mathrm{m})\end{array}$} & \multicolumn{2}{|c|}{ rverage $(\mu \mathrm{m})$} \\
\hline & & $\begin{array}{ll}\text { From } \\
\text { Gas. Per.Test }\end{array}$ & $\begin{array}{ll} & \text { From } \\
\text { Liq. } & \text { Per.Test }\end{array}$ \\
\hline$A L-A$ & 43.18 & $0.171 \pm 0.006$ & $0.174 \pm 0.004$ \\
\hline$A L-B$ & 40.64 & $0.169 \pm 0.010$ & $0.172 \pm 0.002$ \\
\hline$A L-C$ & 40.64 & $0.172 \pm 0.004$ & $0.170 \pm 0.003$ \\
\hline$A L-D$ & 40.64 & $0.174 \pm 0.005$ & $0.172 \pm 0.005$ \\
\hline
\end{tabular}

a. Each value is averaged from 4 observations. 
permeability tests were in close agreement, and these values were somewhat lower than the nominal diameter specified by the manufacturer. This difference may be due to the difference in the pore diameter measurement method. The bubble point test (110), which counted larger pores over smaller pores, was used in the manufacturer's method. The average value of a pore diameter resulting from our measurements was $0.1715 \mu \mathrm{m}$ with $0.0052 \mu \mathrm{m}$ standard deviation, and the average thickness of the membranes was $41.9 \mathrm{~mm}$. These values were used throughout the later calculations for the boundary layer study.

In Table 5.5, the results of sucrose/water diffusion experiments are presented along with the estimated values of total transfer resistances. Also in Table 5.6, similar results with $\mathrm{n}$-octane/n-decane are summarized. As shown in Tables 5.5 and 5.6, the total transfer resistances estimated with the empirical equation (Equation 5.5) for the boundary layer resistance do not match well with the total transfer resistances from the experiments.

Since it was assumed that alumina membranes had an ideal pore structure, i.e. complete cylindrical pores, and that the boundary layer behavior was the same as that achieved by using the track-etched polymer membranes in the estimation, these observed differences might come from changes in the membrane resistance, in the boundary layer resistance, or in both. 


\section{3}

Table 5.5

Results of Diffusion Experiments with Alumina Membranes (Sucrose (solute)/Water (solvent)), e $25^{\circ} \mathrm{C}$

\begin{tabular}{|c|c|c|c|}
\hline$\underset{\#}{\text { Membrane }}$ & $\begin{array}{l}\text { Stirring } \\
\text { rate } \\
\text { (rpm) }\end{array}$ & $\begin{array}{c}\text { Total transfer } \\
\text { resistance } \\
\text { from experiments } \\
(\mathrm{sec} / \mathrm{cm})\end{array}$ & $\begin{array}{l}\text { Estimated } \\
\text { total transfer } \\
\text { resistance } \\
(\mathrm{sec} / \mathrm{cm})^{\mathrm{a}}\end{array}$ \\
\hline$A L-G$ & 100 & 3,704 & 3,312 \\
\hline$A L-H$ & 100 & 3,635 & 3,312 \\
\hline$A L-F$ & 200 & 2,824 & 2,502 \\
\hline$A L-F$ & 200 & 2,896 & 2,502 \\
\hline$A L-F$ & 300 & 2,868 & 2,178 \\
\hline$A L-F$ & 300 & 2,587 & 2,178 \\
\hline$A L-G$ & 300 & 2,703 & 2,178 \\
\hline$A L-H$ & 300 & 2,822 & 2,178 \\
\hline$A L-H$ & 300 & 2,880 & 2,178 \\
\hline$A L-F$ & 400 & 2,550 & 1,997 \\
\hline$A L-F$ & 400 & 2,414 & 1,997 \\
\hline$A L-\bar{F}$ & 500 & 2,356 & 1,879 \\
\hline$A L-H$ & 500 & 2,443 & 1,879 \\
\hline$A L-F$ & 800 & 2,183 & 1,681 \\
\hline$A L-F$ & 800 & 2,259 & 1,681 \\
\hline$A L-G$ & 800 & 2,215 & 1,681 \\
\hline
\end{tabular}

a. Boundary layer resistances were estimated from Equation 5.5. 
Table 5.6

Results of Diffusion Experiments with Alumina Membranes (n-octane (solute)/n-decane (solvent)), e $25^{\circ} \mathrm{C}$

\begin{tabular}{|c|c|c|c|}
\hline$\underset{\#}{\text { Membrane }}$ & $\begin{array}{l}\text { stirring } \\
\text { rate } \\
\text { (rpm) }\end{array}$ & $\begin{array}{c}\text { Total transfer } \\
\text { resistance } \\
\text { from experiments } \\
\text { (sec/cm) }\end{array}$ & $\begin{array}{l}\text { Estimated } \\
\text { total transfer } \\
\text { resistance } \\
(\mathrm{sec} / \mathrm{cm})^{\circ}\end{array}$ \\
\hline$A L-I$ & 100 & 1,575 & 1,312 \\
\hline$A L-I$ & 100 & 1,532 & 1,312 \\
\hline$A L-I$ & 300 & 1,062 & 890 \\
\hline$A L-I$ & 300 & 1,095 & 890 \\
\hline$A L-I$ & 300 & 1,038 & 890 \\
\hline$A L-I$ & 500 & 944 & 740 \\
\hline$A L-I$ & 500 & 916 & 740 \\
\hline$A L-I$ & 500 & 960 & 740 \\
\hline$A L-I$ & 800 & 861 & 641 \\
\hline$A L-I$ & 800 & 850 & 641 \\
\hline$A L-I$ & 800 & 841 & 641 \\
\hline
\end{tabular}

a. Boundary layer resistances were estimated from Equation 5.5. 
Two approaches have been examined in an attempt to relate these observations to the properties of alumina membranes. One was rodification of membrane resistance by introducing a tortuosity factor in alumina membranes, and the other was modification of the boundary layer resistance by assuming the tortuosity factor of alumina membranes is equal to unity. The tortuosity factor for the alumina membranes was obtained by plotting the membrane resistances from the experiments against the membrane resistances from the theoretical equation $\left(1 / D_{0} n \pi r_{0}^{2}\right)$. The experimental membrane resistance was calculated by subtracting the estimated boundary layer resistance from the total transfer resistance in each experiment. The boundary layer resistance was estimated from Equation 5.5. In Figure 5.6, the least square plot of these points is presented. The tortuosity factor of the alumina membrane obtained from the slope of Figure 5.6 was $1.38 \pm 0.022$.

As a second approach, an empirical equation describing the boundary layer behavior in diffusion experiments with alumina membranes was developed by applying the same technique used in the previous boundary layer study with the track-etched polymer membranes. In Figure 5.7, $\operatorname{Ln}\left(\mathrm{N}_{\mathrm{sh}} / \mathrm{N}_{\mathrm{sc}}{ }^{0.33}\right)$ vs. In $\left(\mathrm{N}_{\mathrm{Re}}\right)$ is plotted. The slope and intercept give the exponent term of the Reynolds number and the constant term in the correlating empirical equation, respectively. The resulting equation is: 


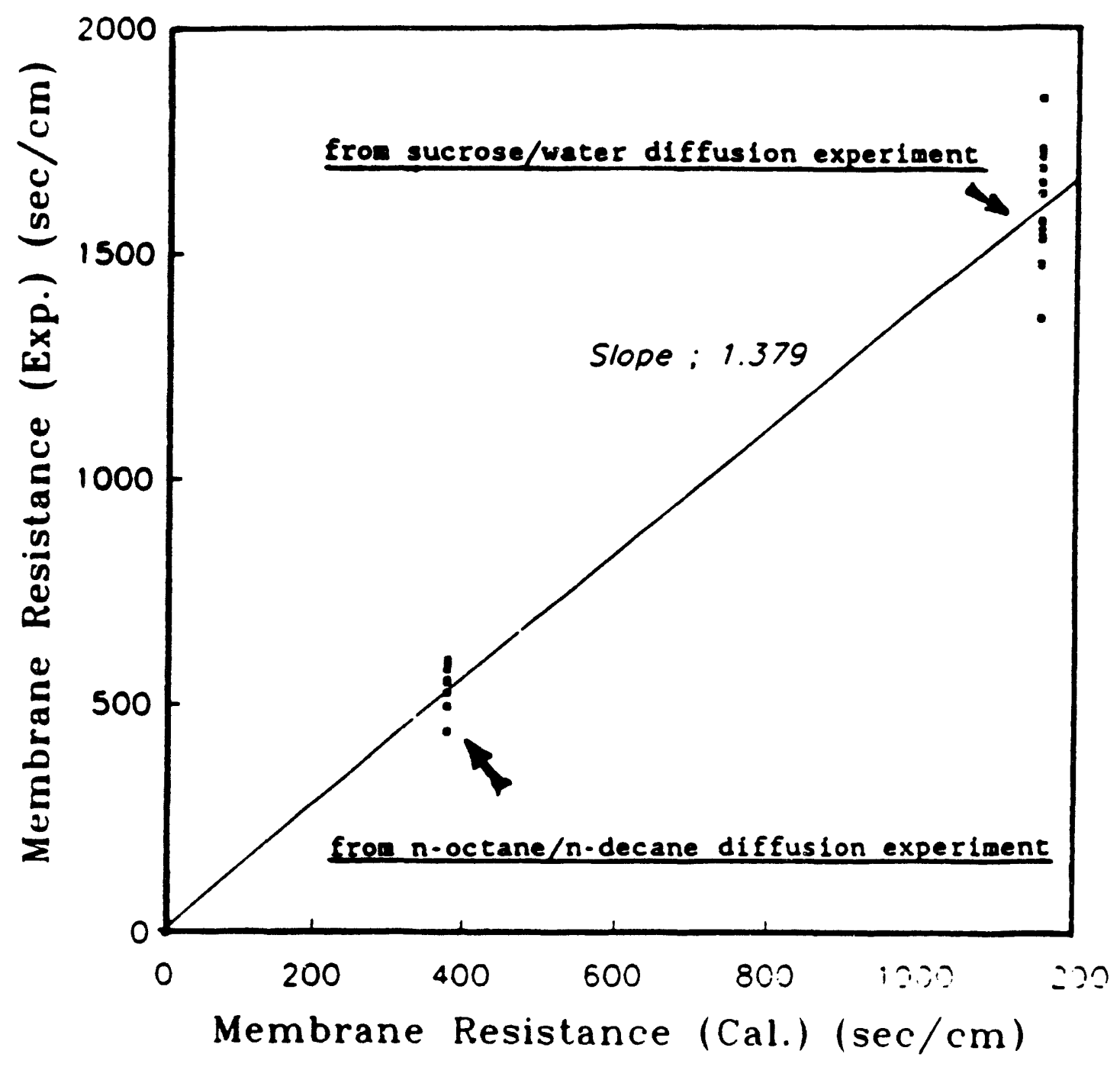

Figure 5.6 Estimation of the Tortuosity Factor of Alumina Membranes. 


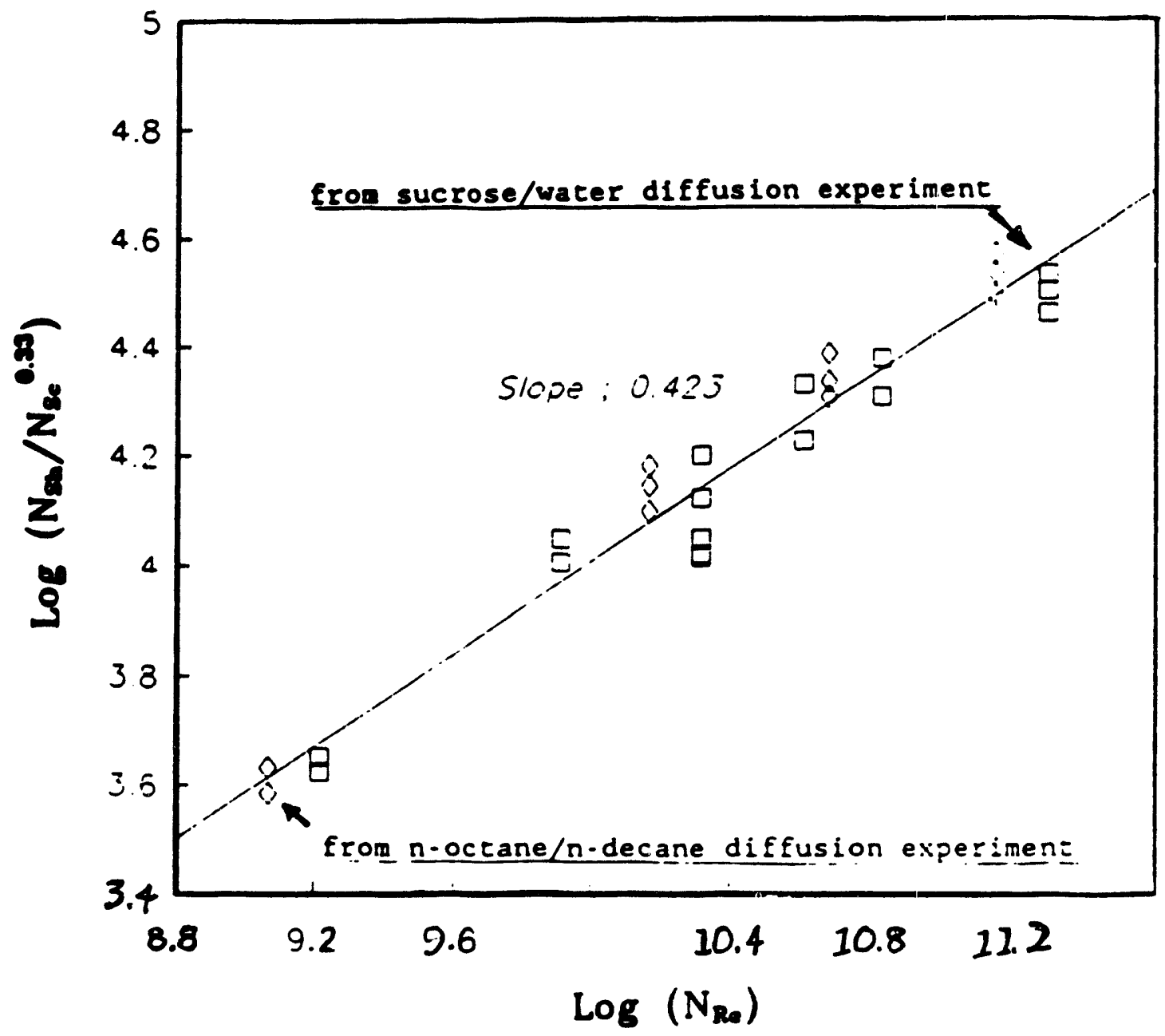

Figure $5.7 \quad \mathrm{~N}_{S h}$ vs. $\mathrm{N}_{\text {Re }}$ Correlation for Diffusion Data with Alumina Membranes. 


$$
\mathrm{N}_{\text {sh }}=0.8 \quad \mathrm{~N}_{\mathrm{Re}}^{0.42} \mathrm{~N}_{\mathrm{Sc}}^{0.33}
$$

In this empirical equation, one point to consider is that sherwood number dependence on the Reynolds number is somewhat lower here than it was observed to be in similar studies. The exponent terms of the Reynolds number varies from 0.5 to 1.0 in the other theoretical and experimental studies $(28,31-34)$.

Thus, it can be concluded that introducing the tortuosity factor in alumina membranes is better than using the new empirical equation for the boundary layer resistance to interpret the experimental data with alumina membranes for the following reasons: (1) the exponent of the Reynolds number in the new empirical equation (Equation 5.6) is unrealistic: and (2) differences between experimental data and estimated values for the total transfer resistances in Tables 5.4 and 5.5 show little dependence upon the stirring rate.

This conclusion was confirmed later during the diffusivity measurement of $n$-octane in ethyl acetate as described in chapter VI. The diffusivity value of $n$-octane cbtained from the riffusion experiments using alumina membranes was almost the same as the value obtained from diffusion experiments using track-etched polyester membranes. Since membrane properties were not directly used in the analysis of the experimental data (the indirect measurement method) and the same empirical equation 


\section{9}

describing the bounciary layer resistance was used, the result implied that the boundary layer behaviors were the same in diffusion experiments using both membranes. However, introducing the tortuosity factor in the alumina membrane needs more careful consideration, since our characterization of alumina membranes was not complete. Variations in pore density, distribution of pore diameter, changes in pore cross sectional area, and shapes of pores all might affect our calculation of the estimated membrane resistance. 


\section{DIFFUSION OF MODEL COMPOUNDS}

Prior to the hindered diffusion study of coal macromolecules, several model compound diffusion studies were conducted in order to test the performance of experimental system and to provide a basic understanding about the diffusional phenomena of some coal model molecule.

Diffusion studies of model compounds consist of four parts. In the first part of this study, paraffinic hydrocarbon diffusion experiments were conducted to test the performance of the diffusion experimental system. In the second part, the diffusion coefficient of n-octane in ethyl acetate, which was used as a reference to calculate the diffusivities of polycyclic aromatic nydrocarbons and coal macromolecules, was measured. In the third part, the diffusion coefficients of polycyclic aromatic hydrocarbons, representing typical constituents in a coal system, were measured. And in the last part, a hindered diffusion study of a model macromolecule compound was performed. In this last study, tetraphenylporphine (TPP) was used as a model compound since the molecular weight of TPP was in the molecular weight range of coal asphaltene and the structural shape of TPP (a thin disk shape) somewhat resembled that of coal asphaltene (stacks of polycyclic aromatic rings). 


\section{Diffusion Experiments with Paraffinic Hydrocarbons}

Experiments

Three paraffinic hydrocarbons, n-octane, n-dodecane and n-tetradecane, were used as solutes in this set of experiments and were supplied by Aldrich Chemical Co. (Milwaukee, WI). N-decane, used as a solvent, was purchased from Fisher Scientific Co. (Pittsburgh, PA). All hydrocarbons were over $99 \%$ purity and were used directly in all diffusion experiments without any further purification. Experiments were conducted with Nuclepore ${ }^{T M}$ polycarbonate membranes having the smallest nominal pore size $(0.01 \mu \mathrm{m})$. The average actual pore size of these membranes was $0.027 \mu \mathrm{m}$ as seen Table 3.2. Using the small pore-sized membranes in the diffusion experiments had two advantages for improving experimental accuracy; one was that it put more emphasis to the membrane transport, and the other was that it reduced the magnitude of errors arising from the time required for the sampling and injecting of solutes because of a relatively longer diffusion time. However, the hindrance factor became significant as the pore size decreased. Thus, this factor was considered in the analyses of experimental data.

All the experimental procedures were the same as the ones described in Chapter IV. As noted in chapter IV, the injection points of solutes were changed depending on the specific gravities of hydrocarbons in order to avoid any 
possible prediffusion of solutes before the diffusion times were counted. $\mathrm{N}$-octane was injected into the upper compartment of the diffusion cell, while n-dodecane and ntetradecane were injected into the bottom compartment. The amount of solute introduced to the diffusion cell in each experiment was about $0.3 \mathrm{ml}$.

In this experimental set, the diffusivities of solutes (n-dodecane and $n$-tetradecane) were also measured using an indirect measurement method. In the indirect measurement method, a mixture (usually, 50/50 wt $z$ ) of a hydrocarbon with unknown diffusivity (n-dodecane or n-tetradecane) and a hydrocarbon with known diffusivity (here, n-octane) was injected into the bottom compartment, and the unknown diffusion coefficients of hydrocarbons were analyzed by the use of the diffusion coefficient of n-octane as a reference value. This method has a relative advantage of eliminating the membrane characterization step, and yet still gives a fairly accurate result, as will be discussed later.

All concentration analyses were carried out by gas chromatography, and toluene was used as an internal standard throughout these experiments. Operating conditions of the G. C. for each measurement were basically the same as those used in the boundary layer study, except for the column temperature control. In Table 6.1, the column temperature settings and the resulting response factor for each experimental set are summarized. 
Table 6.1

G. C. Column Temperature Condition for Each Experiment

\begin{tabular}{|c|c|c|}
\hline Solutes & $\begin{array}{l}\text { Temperature } \\
\text { setting of } \\
\text { column }\end{array}$ & Response factor \\
\hline n-dodecane & $\begin{array}{l}150^{\circ} \mathrm{C} \\
\text { isothermal }\end{array}$ & 1.1396 \\
\hline n-tetradecane & $\begin{array}{l}150{ }^{\circ} \mathrm{C} \\
\text { isothermal }\end{array}$ & 1.0768 \\
\hline $\begin{array}{l}\text { n-octane \& } \\
\text { n-dodecane }\end{array}$ & $\begin{array}{l}90{ }^{\circ} \mathrm{C}: 11 \text { min } \\
15{ }^{\circ} \mathrm{C} / \mathrm{min} . \\
\text { until } 150{ }^{\circ} \mathrm{C} \\
\text { programmed }\end{array}$ & $\begin{array}{l}c_{8}: 1.107 \\
c_{12}: 1.067\end{array}$ \\
\hline $\begin{array}{l}\text { n-octane \& } \\
\text { n-tetradecane }\end{array}$ & $\begin{array}{l}90{ }^{\circ} \mathrm{C}: 12 \mathrm{~min} \\
10{ }^{\circ} \mathrm{C} / \mathrm{min} . \\
\text { until } 150{ }^{\circ} \mathrm{C} \\
\text { programmed }\end{array}$ & $\begin{array}{l}c_{8}: 1.122 \\
c_{12}: 1.060\end{array}$ \\
\hline
\end{tabular}


Results and Discussion

After the diffusion experiments, the total transfer resistances $\left(R_{T}\right)$ were calculated from the experimental results using Equation 2.1. Once the total transfer resistance has been calculated, the diffusion coefficient of a model hydrocarbon was evaluated using Equation 6.1 with membrane property information.

$$
\left.D_{-}=\frac{1}{\left(R_{T}-2 R_{b}\right) \cdot\left(n \pi r_{0}^{2}\right) \cdot\left(1+\left(\frac{9}{8}\right)\left(\frac{I_{s}}{r_{0}}\right) \ln \left(\frac{I_{s}}{r_{0}}\right)-1.54\left(\frac{I_{g}}{r_{0}}\right)\right)} ; 6.1\right)
$$

Equation 6.1 was obtained by rearranging Equation 2.2 in terms of the bulk diffusivity $\left(D_{0}\right)$. In this equation, the terms in the third bracket of the denominator represent the hindrance factor proposed by Branner and Gaydos (40) (Equation 2.20 in Chapter II). Since the ratios of molecular to pore radii were much smaller than 0.1 in this experimental set, using the hindrance factors derived from Equation 2.20 was expected to be adequate for the current study. In the analysis of the experimental data using Equation 6.1 , the molecular radius of $n$-octane $\left(r_{s}\right)$ was obtained by the use of stokes-Einstein equation. However, the stokes-Einstein radius of solute is related to the bulk diffusivity of solute $\left(r_{s}=k \cdot T /\left(6 \pi \cdot \mu \cdot D_{*}\right)\right)$. The bulk diffusivity of solute $\left(D_{\infty}\right)$ was also expressed implicitly in the boundary layer resistance term $\left(R_{b}\right)$ as seen in Equation 5.5. Thus, solving Equation 6.1 to obtain the bulk 
diffusivity from experimental data required a root-finding method. Eureka Equation Solver package (Borland International, Inc., Scotts Valley, CA) was used to solve this nonlinear equation.

In Table 6.2, measured values of each hydrocarbon diffusion coefficient are summarized along with the corresponding literature values calculated from the estimation equations reported by Lo (80) and Hayduk et al. (81). The results show general agreement for the diffusion coefficient of each hydrocarbon, compared to the estimated values in the literature.

In the indirect measurement method, the total transfer resistance of a compound with unknown diffusivity was calculated along with the total transfer resistance of a known compound from the multicomponent ternary diffusion experimental data. In this calculation, the diaphragm cell diffusion equation for multicomponents was analogized into the corresponding binary problem. This approach, used in the calculation, was reasonable, since the concentration range of solute is quite low (less than 0.5 wto) anc the paraffinic hydrocarbons form a noninteracting solution. with these experimental total transfer resistances, the unknown diffusion coefficients of any compound can be calculated from the following equation: 
Table 6.2

Summary of Measured Diffusion Coefficients for $\mathrm{N}$-paraffinic Hydrocarbons in: $\mathrm{N}$-decane $225^{\circ} \mathrm{C}$.

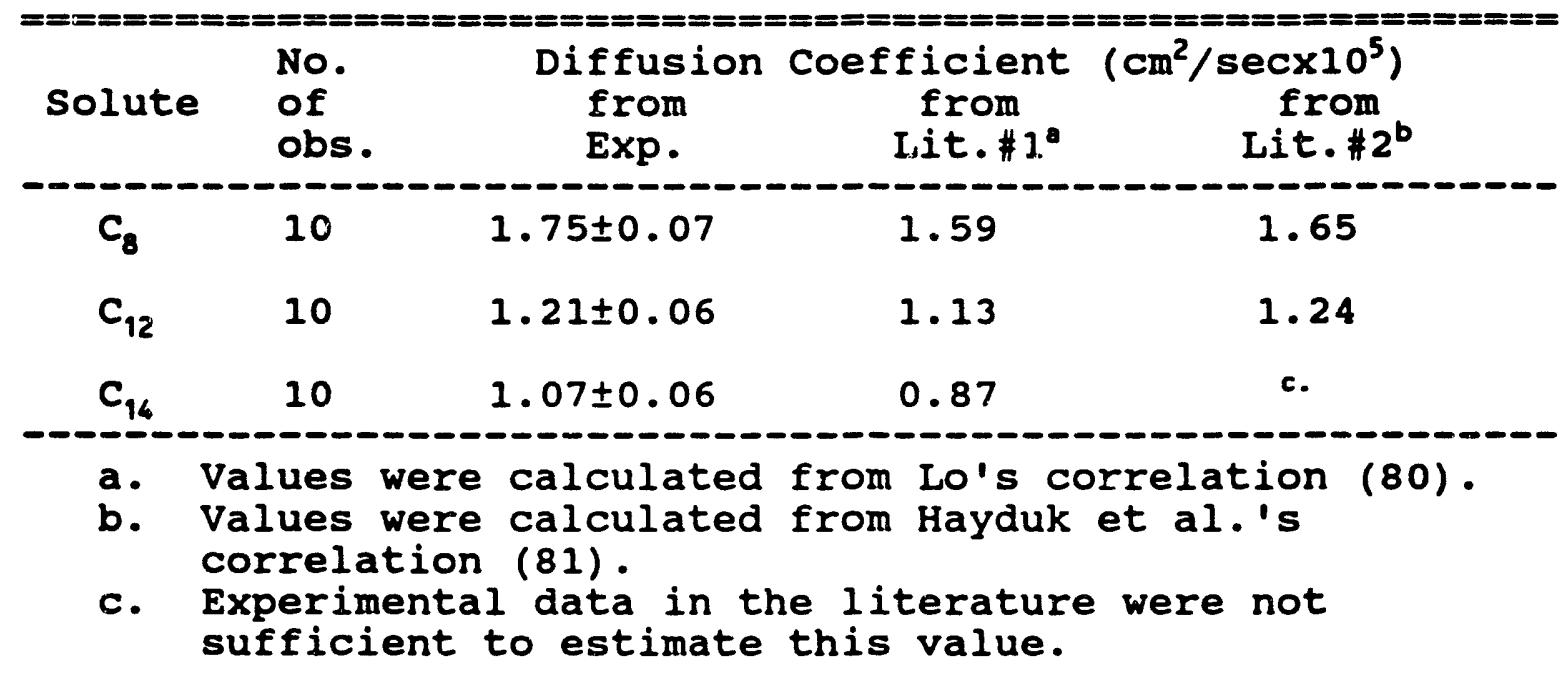




$$
\frac{D_{a_{1}}}{D_{a_{2}}}=\frac{R_{B_{2}} \cdot f\left(\lambda_{2}\right)}{R_{m_{1}} \cdot f\left(\lambda_{1}\right)}=\frac{R_{T_{2}}-2 R_{b_{2}}}{R_{T_{1}}-2 R_{b_{1}}} \cdot \frac{f\left(\lambda_{2}\right)}{f\left(\lambda_{1}\right)}
$$

where,

$$
f\left(\lambda_{i}\right)=1+\left(\frac{9}{8}\right)\left(\lambda_{i}\right) \ln \left(\lambda_{i}\right)-1.539 \lambda_{i}, \quad \lambda_{i}=\frac{r_{s_{i}}}{r_{0}}
$$

Solving this equation to obtain the unknown diffusivity of a solute also required a root-finding method for the same reasons associated with solving Equation 6.1 .

A reference bulk diffusivity $\left(D_{02}\right)$ used in this calculation was the diffusivity of n-octane in $n$-decane. The estimated diffusivity of n-octane as used in the boundary layer study was $1.62 \times 10^{-5} \mathrm{~cm}^{2} / \mathrm{sec}$, the value which was the average of the values calculated from the Lo's correlation and from the Hayduk et al.'s correlation, while the experimental value measured in the current study was $1.75 \times 10^{.5} \mathrm{~cm}^{2} / \mathrm{sec}$. Both values were used as the reference diffusivities to calculate the bulk diffusivities of $n$ dodecane and n-tetradecane using Equation 6.2 with the experimental data. The results are summarized in Table 6.3 along with the results from the direct measurements and the estimated values from Lo's correlation $(80)$. It appears that the diffusivity values of $n$-dodecane and $n$-tetradecane obtained from the indirect measurement method agree with those from the direct measurement method.

In comparing the use of the experimental diffusivity of n-octane with that of the estimated diffusivity as a 
Table 6.3

Summary of Diffusion Coefficients irom the Direct and Indirect Measurements

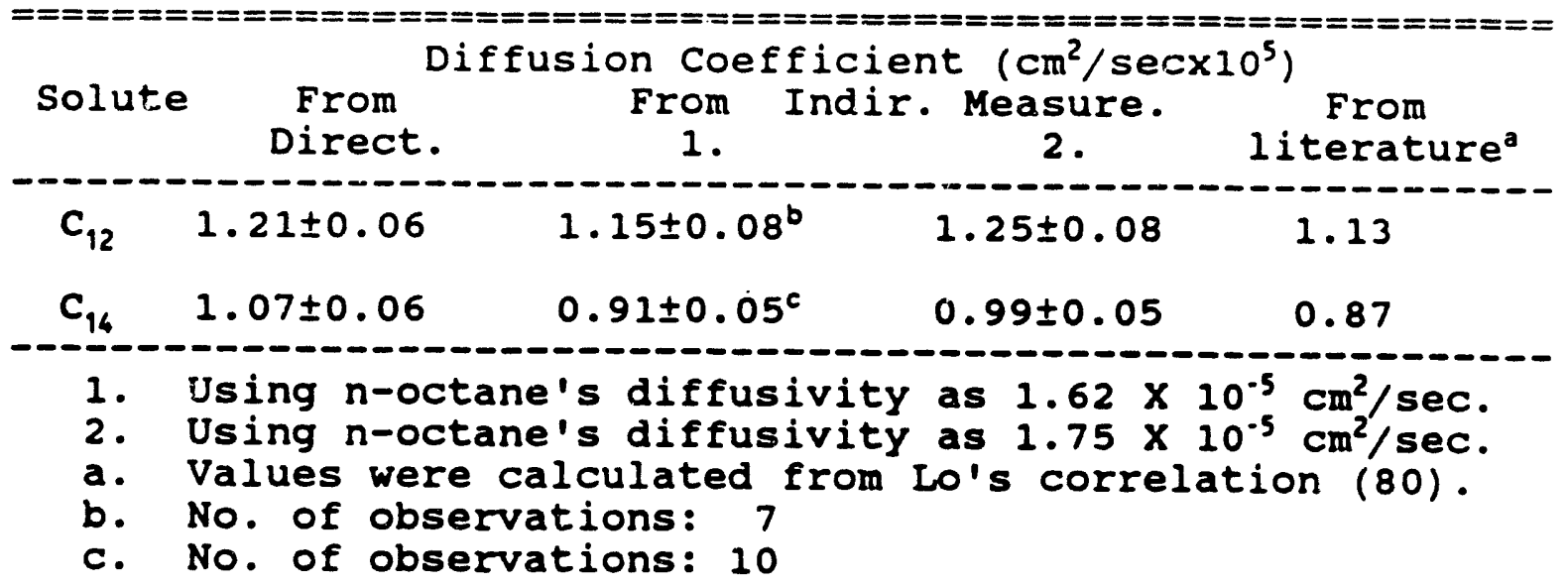


reference value, employing the estimated diffusivity of $n$ octane $\left(1.62 \times 10^{-5} \mathrm{~cm}^{2} / \mathrm{sec}\right)$ yielded closer results between the experimental values and the predicted values for the diffusivities of $n$-dodecane and $n$-tetradecane.

since the diffusivity of n-octane in $n$-decane was used as a reference value in many indirect diffusivity measurements used in the current research, selection of $n$ octane's diffusivity between the experimental value and the estimated value required a careful consideration. For the following reasons, the estimated value was used throughout the current study; (1) The correlation equations used for the estimations were derived from many diffusion data of paraffinic hydrocarbon systems (i.e., both solute and solvent were paraffinic hydrocarbons). Thus, these equations appeared to predict the actual experimental data precisely (average errors of Lo's correlations and Hayduk et al.'s correlation: $4.2 \%, 1.8 \%$ respectively, as described in Appendix D). (2) In the direct measurement of n-octane's diffusivity, two independent experiments were required. One was a He gas permeability measurement to determine the average pore size of membrane and the other was the diffusion experiment to obtain the total transfer resistance. Consequently, the overall experimental error associated with the direct measurement method was expected to be relatively high. (3) As shown in Appendix E, the hindrance factor varies very sharply depending on a ratio of 
molecule to pore size. (i.e., a small error in pore size measurements or diffusion experiments might cause a significant change in the hindrance factor). (4) The derivations of the equations used in the direct measurement method involved several assumptions which were approximations of the actual situations.

Although the bulk diffusivity of n-octane obtained from the current study was close enough to the estimated value within an experimental error, it was concluded that the use of the estimated value $\left(1.62 \times 10^{-5} \mathrm{~cm}^{2} / \mathrm{sec}\right)$ would be more conservative for the current study.

As noted previously, the agreement between the diffusivity values obtained from the direct measurement method and the indirect measurement method indicates that the binary system analogy for the two paraffinic hydrocarbon diffusion experiments is reasonable. The results are also important in the diffusion study of coal asphaltene, since the diffusion of coal asphaltene is basically multicomponent diffusion, and binary system analogy of the multicomponent diffusion process makes the analyses of multicomponent experiments' results much easier.

\section{Selection of Solvent and Diffusivity Measurements} for $\mathrm{N}$-octane in Ethyl Acetate

Coal asphaltene is defined by its solubility characteristics (soluble in an aromatic solvent such as benzene or toluene but insoluble in light paraffinic 
hydrocarbons such as pentane or heptane). Thus, to conduct the hindered diffusion experiments of coal macromolecules, solvent having a reasonable solubility of coal asphaltene and being chemically compatible with membranes used in the experiments should be chosen. In addition, safety (volatility, toxicity, carcinogenic nature) was also considered in the selection of a solvent.

In these respects, ethyl acetate was selected as a sclvent for the hindered diffusion study of coal macromolecules for the following reasons. Filrst, it has similar solubility characteristics to that off aromatic hydrocarbons (111-113). Because of this characteristic, ethyl acetate had been adopted to determine the conversion of coal to liquefaction product (oil plus asphaltene fraction) in previous studies $(114,115)$. Second, ethyl acetate was fully chemically compatible with the polyester membranes which were used in the hindered diffusion study of coal macromolecules. Third, ethyl acetate is not a carcinogenic material, while the aromatic hydrocarbon (benzene) used for the fractionation of coal asphaltene has a carcinogenic nature.

As described in Chapter III, the pore sizes of membranes used in the hindered diffusion of coal macromolecules were determined from diffusion experiments. In these experiments, the diffusivity of n-octane in ethyl acetate was used to calculate the pore radius with 
experimental data. This experimental value for the diffusivity of n-octane was not available in the literature. Thus, it was obtained from the experimental measurements using a method similar to the indirect measurement method.

\section{Experiments}

Ethyl acetate (optima grade) and $n$-decane $(998+)$ was obtained from Fisher scientific Co. N-octane (998+) was supplied by Aldrich Chemical Co. In the experiments, two sets of experiments using two different membranes were conducted; one kind of membrane used in these experiments was a commercial alumina membrane (nominal pore diameter:

$0.2 \mathrm{\mu m}$ ) purchased from Anotec separation Co., and the other kind of membrane was a track-etched polyester membrane (approximate pore diameter: $0.08 \mathrm{~mm}$ ) prepared in the author's laboratory.

The experimental method for the diffusivity measurement was similar to the indirect measurement method; for the same membrane, experiments were carried out by switching the solvents, i.e., one with n-decane and the other with ethyl acetate.

The diffusion coefficient of n-octane in ethyl acetate was calculated from the total transfer resistances obtained from two different solvent experimental systems using Equation 6.2 and the diffusion coefficient of n-octane in $n$ decane determined from the previous study as a reference value. In the analyses of the experimental data, the 
hindrance factors were not counted since the membranes used in this study had sufficiently large pores, which would not cause any appreciable hindrance effect.

Results and Discussion

Diffusion data for the experiments with alumina membranes and polyester membranes are summarized in Tables 6.4 and 6.5 , respectively. Each value in this table is the total transfer resistance calculated from Equation 2.1 with the experimental result. As seen in this table, all diffusion data were quite reproducible.

with the total transfer resistances obtained from experiments, the empirical equation for the boundary layer resistance (Equation 5.5) and the estimated diffusivity of $n$-octane in $n$-decane $\left(1.62 \times 10^{-5} \mathrm{~cm}^{2} / \mathrm{sec}\right)$ for the reference value, the diffusion coefftcients of n-octane in ethyl acetate were calculated using Equation 6.2. The results are presented in Table.6. As shown in the table, the experimental values obtained from two different sets of experiments were in close agreement. Also, these values were reasonably close to the predicted values calculated from several estimation methods in the literature (84-87). The average of all experimental diffusivity values of $n$ octane in ethyl acetate was found to be $2.47 \times 10^{-5} \mathrm{~cm}^{2} / \mathrm{sec}$. In an effort to verify the conclusions drawn about the boundary layer behavior in the diffusion experiments with alumina membranes, the diffusion coefficients of n-octane in 
Table 6.4

Results of Indirect Diffusion Coefficient Measurements $\left(\mathrm{C}_{8}\right.$ (solute) $/ \mathrm{n}$-decane and ethyl acetate (solvınt)) e $25^{\circ} \mathrm{C}$ with Alumina Membranes

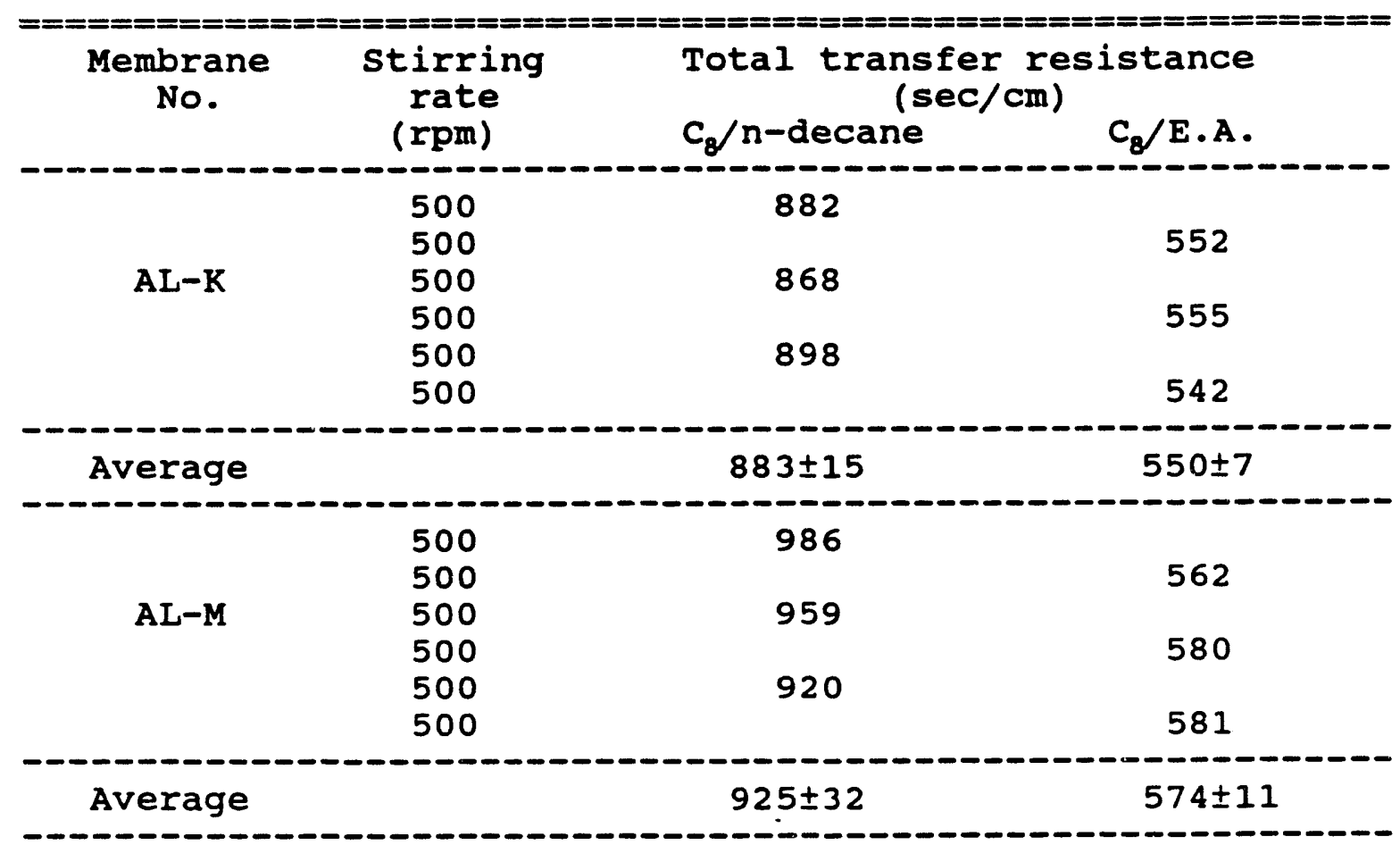


Table 6.5

Results of Indirect Diffusion Coefficient Measurements $\left(\mathrm{C}_{8}\right.$ (solute) / $\mathrm{n}$-decane and ethyl acetate (solvent)) $25^{\circ} \mathrm{C}$ with Polyester Membranes

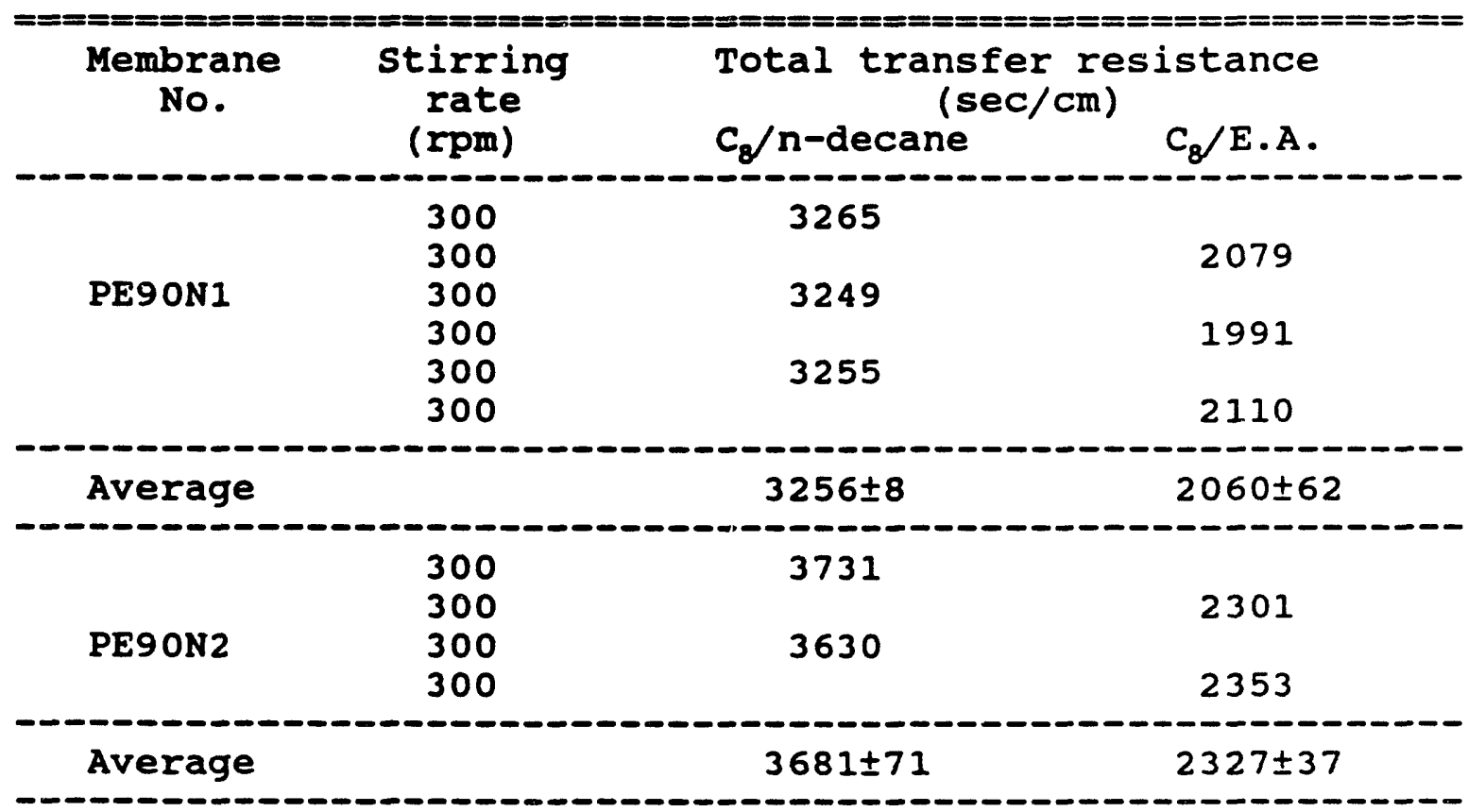


Table 6.6

Diffusion Coefficients for $\mathrm{N}$-octane in Ethyl acetate $25^{\circ} \mathrm{C}$

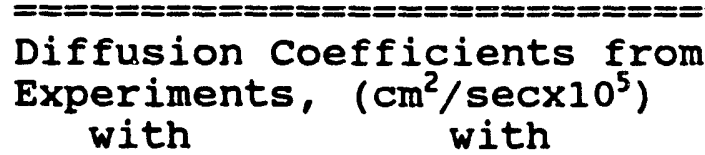

Alumina M. Polyester $M$.

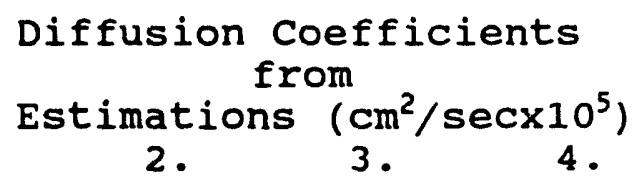

$2.44 \pm 0.01 \quad 2.49 \pm 0.01 \quad 2.04^{\mathrm{a}, \mathrm{b}} 2.34^{\mathrm{c}} 2.09^{\mathrm{d}} 2.61^{\mathrm{e}}$
a. Molar volumes at normal boiling point temperature were
estimated by using Tyn and Calus method (88).
b. Wilke \& Chang Method $(84)$
c. Reddy \& Doraiswamy Method (85)
d. Lusis \& Ratcliff Method (86)
e. King, Hsueh and Mao Method (87)


ethyl acetate obtained by using two different empirical equations for the boundary layer resistance (Equation 5.5 and Equation 5.6) were compared. The results are presented in Table 6.7. As seen in this table, the diffusion coefficient resulting from using Equation 5.6 is much different from the other values. In other words, it appeared that the boundary layer behavior in the diffusion experiment with alumina membranes was the same as the one with track-etched polyester membranes. Thus, the results supported the conclusion drawn in chapter $\mathrm{V}$.

\section{Diffusion Experiments with Polycyclic Aromatic Hydrocarbons}

The organic materials in coal consist of complex macromolecules having high aromatic content. It has been reported that the most predominant constituents of coal extract are the polycyclic aromatic hydrocarbons (83). Yet the experimental values of diffusivity in ethyl acetate for the most of these materials were not available in the literature. Thus, the diffusion coefficients of some of these materials were measured to increase an understanding of the diffusional behavior of coal macromolecules.

\section{Experiments}

Ten model compounds were selected among the polycyclic aromatic hydrocarbons. The number of aromatic rings in these model compounds ranged from two to eight. These were: naphthalene $(99+\%)$, anthracene $(99+\%)$, phenanthrene (99.5\%) 
Table 6.7

Diffusion Coefficients of $\mathrm{N}$-octane in Ethyl

Acetate from the Different Boundary

Layer Empirical Equations

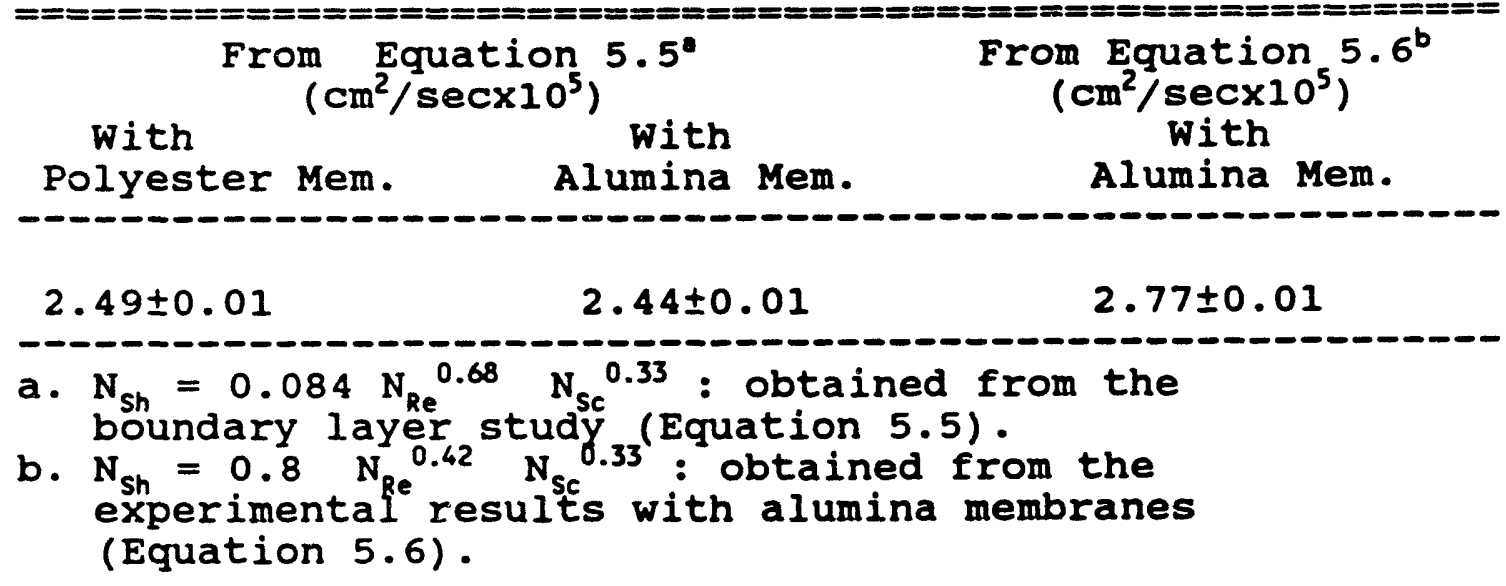


fluorene (98\%), 2,3-benzanthracene (99\%), chrysene (98\%), triphenylene (98\%), pyrene $(99+8)$, coronene (97\%) and rubrene (97\%). All these materials were obtained from Aldrich Chemical co. Ethyl acetate (optima grade) was obtained from Fisher scientific co and used as a solvent throughout the experiments.

All the diffusion experiments were conducted with track-etched polyester membranes which were prepared in the author's laboratory. Average pore diameters of these membranes ranged from about $0.02^{-} \mu \mathrm{m}$ to $0.08 \mu \mathrm{m}$.

Experimental procedures were similar to the ones described in Chapter IV. However, n-octane was also introduced to the top compartment of the diffusion cell to obtain the transfer resistance for the n-octane diffusion during the experiments. The binary system analogy of the multicomponent diffusion process was verified in the previous section. To recheck the validity of this technique, the octane diffusion experiments were conducted separately for some polycyclic aromatic hydrocarbons (naphthalene and pyrene). The results will be discussed later. During the experiments, special consideration was required due to the possible degradation of some polycyclic aromatic hydrocarbons induced by a photo-oxidation reaction. Thus, light was shielded from all experimental apparatus during these experiments. To test the possible degradation of polycyclic aromatic hydrocarbons, the material balance of 
solute was checked for each experiment by subtracting the amount of solute in the diffusion cell after the diffusion experiments from the initial amount of solute introduced into the diffusion cell. Large errors in the material balance provided an evidence of degradation. Actual material balance errors in this set of experiments turned out to be alvays less than $\pm 2 \%$. In these experiments using the indirect measurement method, more than three diffusion experiments were conducted per each model compound diffusion experimental set. The total transfer resistance of the $n$ octane diffusion and the total transfer resistance of each model compound's diffusion were used to calculate the diffusion coefficients of the model compound using equation 6.2. The hindrance factor was counted in the calculation and the diffusivity of octane in ethyl acetate used in this calculation was $2.47 \times 10^{-5} \mathrm{~cm}^{2} / \mathrm{sec}$.

A UV-visible spectrophotometer (Varian Co., Model DMS100S) was used in all concentration analysis of the model compound diffusion experiments. Operating conditions of the UV spectroscopy analysis and the reverse of the extinction coefficients for each model compound are summarized in Table 6.8 .

Results and Discussion

In Table 6.9, results of the diffusion coefficient measurements of 10 polycyclic aromatic hydrocarbons are 
141

Table 6.8

UV operating Conditions for Model Compounds

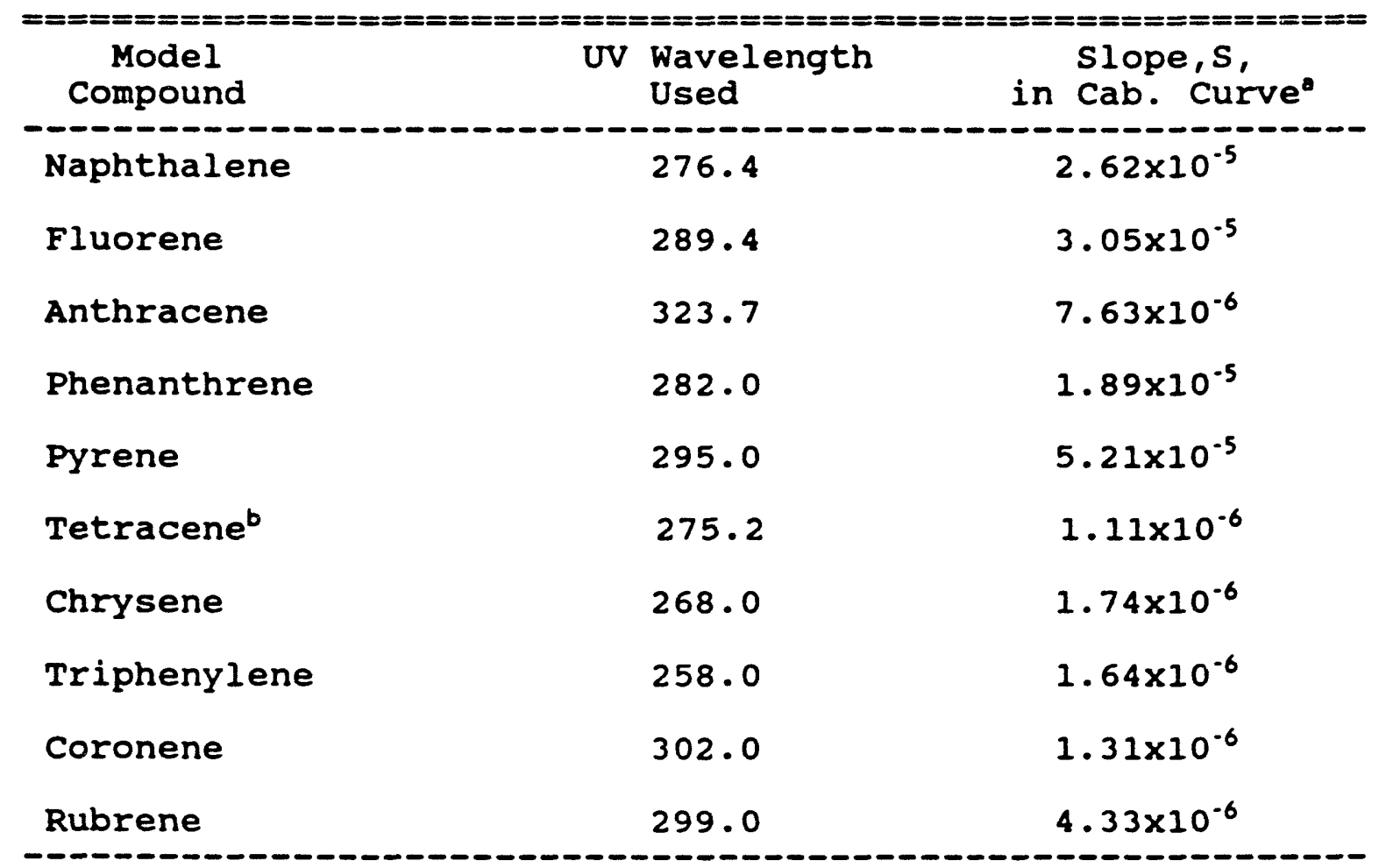

a. Weight fraction of solute in solvent $=S \mathrm{x}$ absorbance

b. 2,3 - benzanthracene 
Table 6.9

Diffusion Coefficients of 10 Polycyclic Aromatic Hydrocarbons in Ethyl Acetate at $25^{\circ} \mathrm{C}$

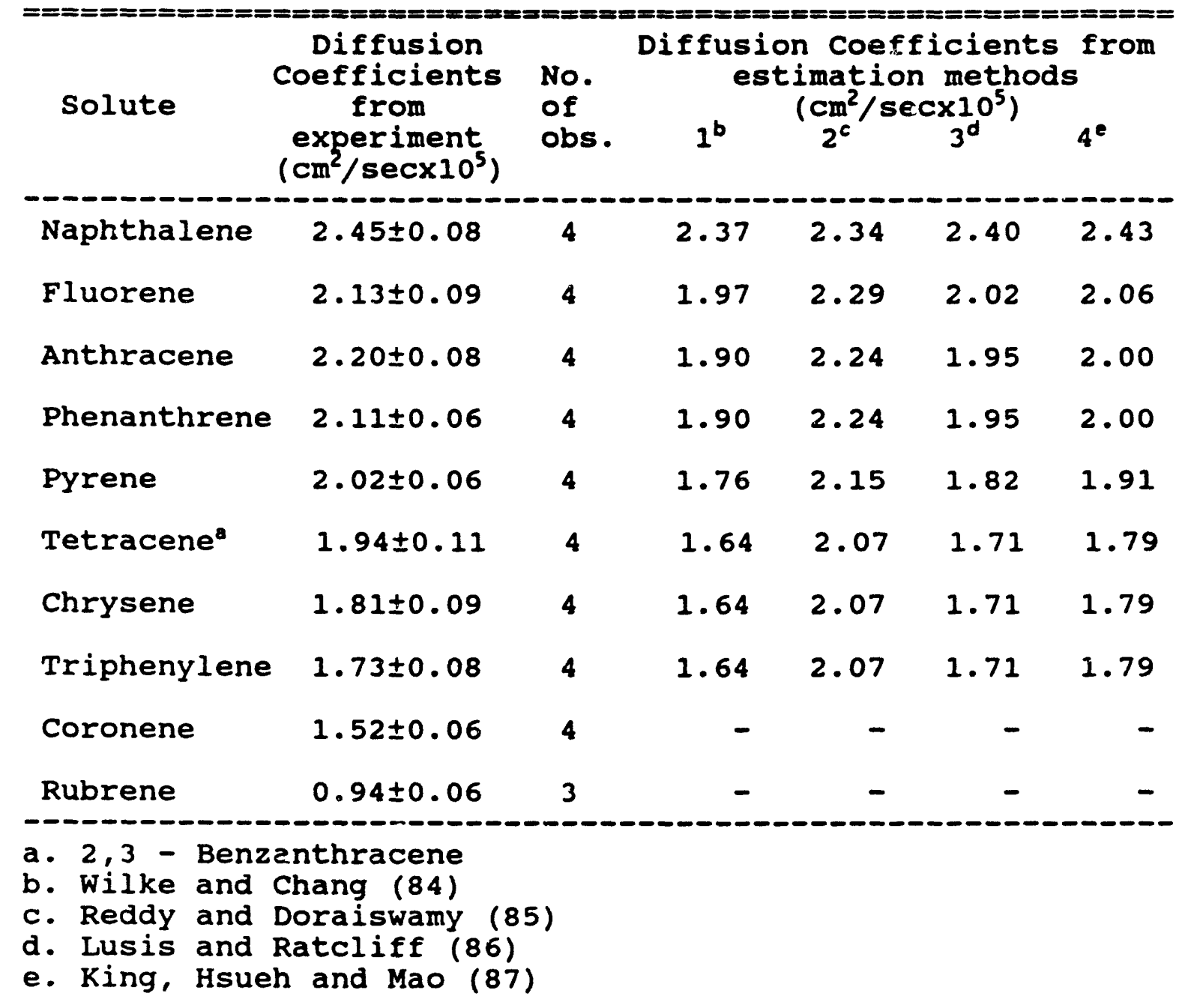


presented along with the predicted values from several estimation methods. As shown in this table, the measured values for the diffusion coefficients of polycyclic aromatic hydrocarbons generally agree with the predicted values. In the calculation of predicted diffusion coefficients, parameters for the molar volumes at the normal boiling points were estimated from the critical volume of each compound as proposed by Tyn and Calus (88), except for naphthalene, for which experimental data was available in the literature (88).

In Table 6.10 , results of the experiments for the separated octane diffusion are presented. As shown in this table, the diffusion coefficients obtained from the in situmeasurement of the octane transfer resistance (octane and polycyclic aromatic hydrocarbons were introduced together) are in close agreement with that obtained from the separated octane diffusion experiment. Thus, the binary system analogy of multicomponent diffusion was also verified in this experimental set.

In Figure 6.1, the measured diffusion coefficients of polycyciic aromatic hydrocarbons are plotted against the number of carbon atoms in each compound. A correlation coefficient for this least square plot was 0.961 . This result can be compared to the similar plot drawn with the diffusion coefficients of paraffinic hydrocarbons. Studies with the n-paraffinic hydrocarbon system have shown that 
Table 6.10

Summary of Diffusion Coefficients Obtained from the in situ-Measurements and the Separated Measurements

\begin{tabular}{|c|c|c|c|c|}
\hline Solute & $\begin{array}{c}\text { No.of } \\
\text { Obs. }\end{array}$ & $\begin{array}{l}\text { Diffusivity } \\
\text { from in situ } \\
\left(\mathrm{cm}^{2} / \sec \times 10^{5}\right)\end{array}$ & $\begin{array}{l}\text { No.of } \\
\text { Obs. }\end{array}$ & $\begin{array}{l}\text { Diffusivity } \\
\text { from Separ } \\
\left(\mathrm{cm}^{2} / \mathrm{sec} 10^{5}\right)\end{array}$ \\
\hline Naphthalene & 4 & $2.43 \pm 0.08$ & 4 & $2.49 \pm 0.12$ \\
\hline Pyrene & 4 & $2.00 \pm 0.04$ & 4 & $1.96 \pm 0.06$ \\
\hline
\end{tabular}




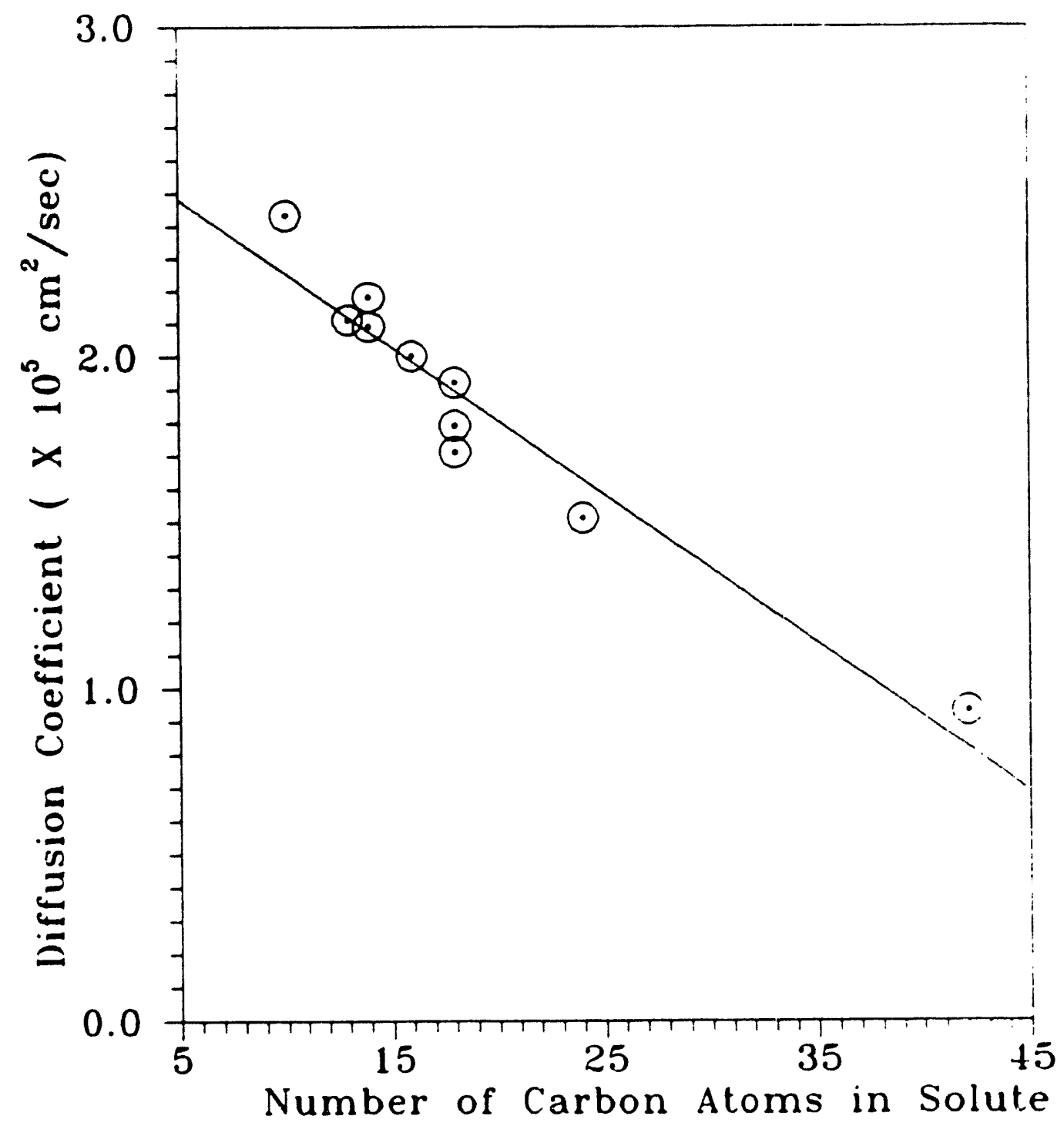

Figure 6.1 Diffusion Coefficients vs. Number of Carbon Atoms in Polycyclic Aromatic Hydrocarbons 
diffusion coefficients of n-paraffinic hydrocarbons can be correlated almost linearly with the number of carbon atoms in $n$-paraffinic hydrocarbons $(80,81)$. Since the configuration of the molecule is one of the important factors which determines the diffusivity of the molecule in liquid phase, this poorer correlation coefficient for polycyclic hydrocarbons is an expected result.

A better linear correlation was obtained when the diffusion coefficients were plotted against the average planar molecular radii as shown in Figure 6.2 . In this figure, the correlation coefficient was 0.978 . Average planar molecular radii were obtained from the molecular models prepared by the molecular modeling software. In the current study, SYBYL (Tripos Associates, Inc., St. Louis, Missouri) was used to construct the molecular models of 10 polycyclic aromatic hydrocarbons. According to these models, most of the aromatic hydrocarbons used in the current study have planar structures (only rubrene has a threedimensional structure). Thus, the sizes of molecules could easily be defined by the longest dimension and the shortest dimension across the molecules. In Table 6.11, these molecular dimensions are summarized. In the table, the axial lengths include Van der Waals' radii of hydrogen atoms. Average molecular radii of molecules used in Figure 6.2 were half the geometric average of the longest planar dimension and the shortest planar dimension of molecules. 


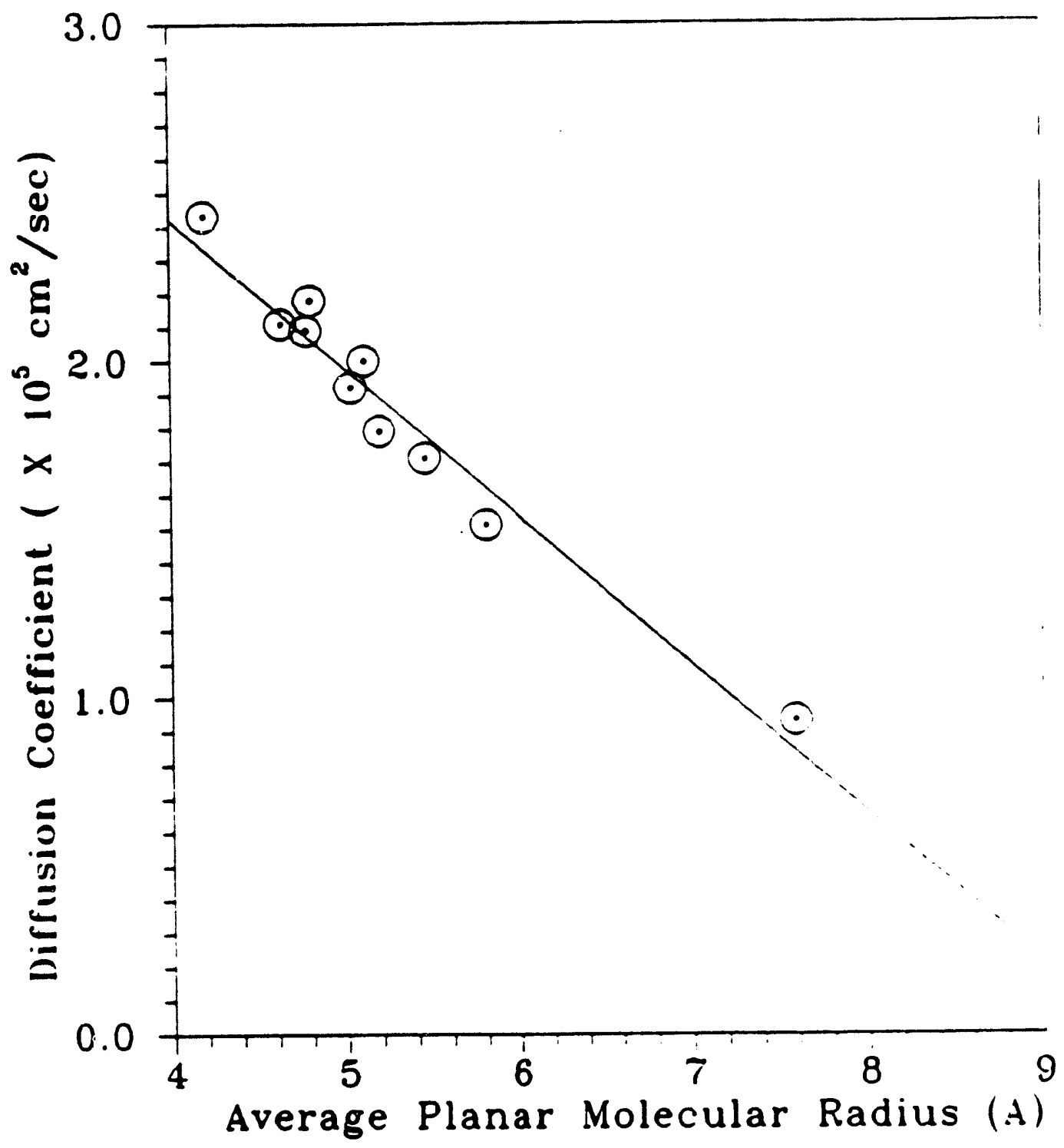

Figure 6.2 Diffusion Coefficients vs. Average Planar Molecular Radius of Polycyclic Aromatic Hydrocarbons 
Table 6.11

Molecular Dimensions of 10 Polycyclic Aromatic Hydrocarbons Determined from Model Work

\begin{tabular}{|c|c|c|c|}
\hline solute & $\begin{array}{c}\text { Molecular } \\
\text { Weight }\end{array}$ & $\begin{array}{l}\text { From } \\
\text { The shortest } \\
\text { Dimension of } \\
\text { Molecules (A) }\end{array}$ & $\begin{array}{l}\text { SYBYL } \\
\text { The Longest } \\
\text { Dimension of } \\
\text { Molecules (A) }\end{array}$ \\
\hline Naphthalene & 128.17 & 7.36 & 9.55 \\
\hline Fluorene & 166.22 & 7.56 & 11.34 \\
\hline Anthracene & 173.23 & 7.36 & 12.54 \\
\hline Phenanthrene & 178.23 & 7.91 & 11.54 \\
\hline Pyrene & 202.26 & 9.04 & 11.54 \\
\hline Tetracene & 228.29 & 7.21 & 14.06 \\
\hline Chrysene & 228.29 & 7.91 & 13.62 \\
\hline Triphenylene & 228.29 & 10.32 & 11.54 \\
\hline Coronene & 300.36 & 11.46 & 11.78 \\
\hline Rubrene & 532.69 & 14.34 & 16.04 \\
\hline
\end{tabular}




\section{Diffusion Experiment with \\ a Model Macromolecule}

Prior to conducting the hindered diffusion experiment with coal asphaltene, diffusion experiments with a model macromolecule were performed. In this study, tetraphenylporphine (TPP) was selected as a model compound because of the closeness of its molecular weight and configuration to coal macromolecules. Porphyrin compounds have been used as a model compound in previous hindered diffusion studies, since they are rigid planar disk-shaped molecules which can be easily characterized $(89,99)$. The structural drawing of TPP is presented in Figure 6.3. The planar dimension of TPP was $18.4 \AA$ (89).

By using the hindered diffusion experimental results of TPP which resembles coal macromolecules, a more rigorous approach can be made in the evaluation of the hindered diffusion experimental results of coal macromolecules in the light of the hindered diffusion theory.

\section{Experiments}

TPP $(99+8)$ was purchased from Aldrich Chemical Co., and ethyl acetate used in these experiments was the same as that used in the previous experiments. Polyester membranes suitable for this study were prepared prior to the diffusion experiment. As shown in Figure 6.3, the plarar dimension of TPP was about $19 \AA$; thus, membranes haviny pore sizes ranging from 75 \& to 468 \& were prepared from irradiated 


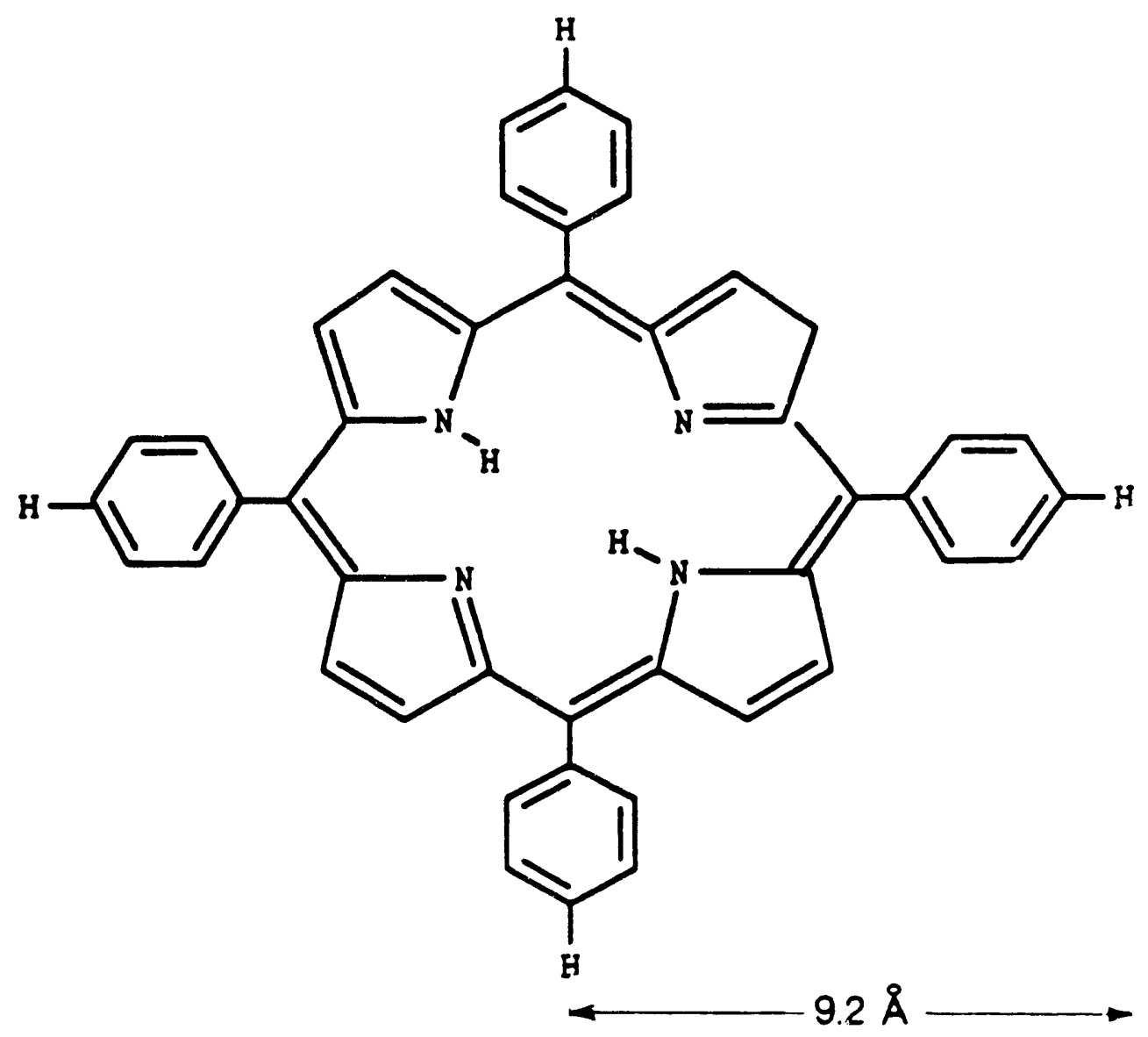

Figure 6.3. Chemical structure of Tetraphenylporphine (89) 
were prepared from irradiated polyester films by an etching process. These films were purchased from Nuclepore corporation. The etching conditions are already described in chapter III. The pore diameters of membranes were determined from the diffusivity measurements of n-octane in ethyl acetate (no TPP was present in these experiments). The bulk diffusion coefficient of TPP was measured with two commercial track-etched polyester membranes having 0.2 $\mu \mathrm{m}$ nominal pore $\mathrm{si}^{-}$.Nuclepore Corporation). The actual pore sizes of these membranes, determined from He gas permeability tests, were found to be $0.239 \mu \mathrm{m}$ and $0.245 \mu \mathrm{m}$, respectively. Although these membranes used in the experiments had large pore size, the hindrance effects were expected to still exist with these membranes because of the molecular dimension of TPP, thus, the hindrance factor was estimated using Equation 2.20 and reflected in the calculation of the bulk diffusivity of TPP.

The concentration of TPP stock solution used for the charging was $117 \mathrm{mg} / \mathrm{l}$. About $0.4 \mathrm{cc}$ of this stock solution was injected into the bottom compartment of the diffusion cell. Thus, the initial concentrations of the bottom compartment of the diffusion cell became about $2.24 \mathrm{mg} / \mathrm{l}$. After the diffusion experiment, concentrations of TPP in the samples were analyzed with a UV-visible spectrophotometer (Varian model DMS 100S). The maximum absorbance wavelength of TPP in ethyl acetate occurred at 


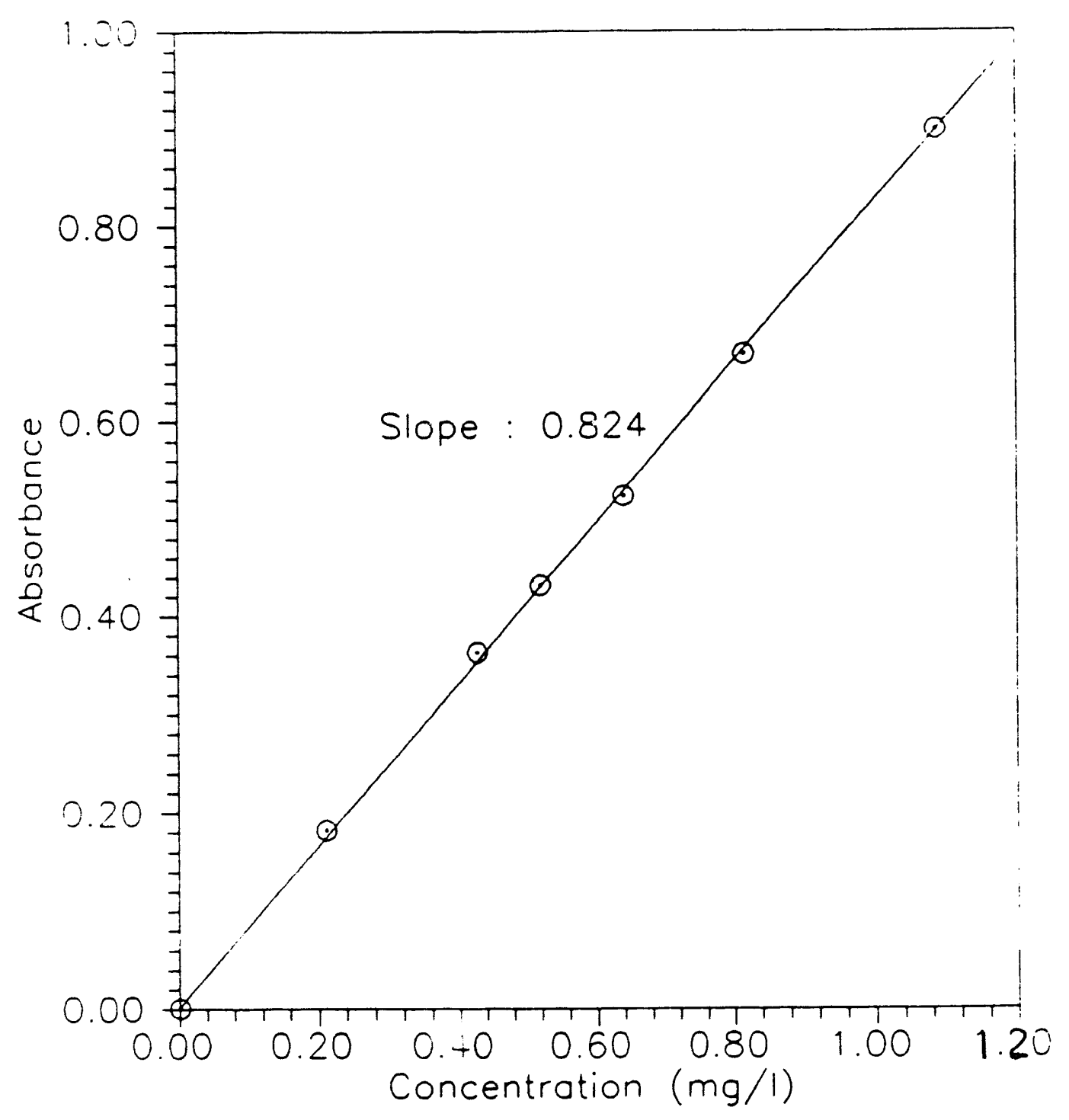

Figure 6.4 UV Calibration Curve for a TPP Solution. (UV Wavelength : $414.3 \mathrm{~nm}$ ) 
$414.3 \mathrm{~nm}$. In Figure 6.4, the calibration curve for the TPP solution is presented.

All other experimental procedures were the same as described in Chapter IV. However, light was shielded from the experimental apparatus in this experimental set to prevent the possible degradation of TPP by light.

\section{Results and Discussion}

In Table 6.12, the experimental results of bulk diffusion coefficient measurements are summarized along with the estimated value. Since the experimental values for the diffusion coefficient of TPP in ethyl acetate were not available in the literature, it was estimated from the diffusion coefficient of TPP in chloroform at $20^{\circ} \mathrm{C}$ measured by Anderson et al. (89) using a quasi-elastic light scattering technique. They reported that the diffusivity of TPP was $5.75 \times 10^{-6} \mathrm{~cm}^{2} / \mathrm{sec}$. To estimate the diffusivity of TPP in ethyl acetate at $25^{\circ} \mathrm{C}$ from this value, the wilkeChang equation was used as shown in Table 7.9. The experimental value $\left(6.83 \times 10^{-6} \mathrm{~cm}^{2} / \mathrm{sec}\right)$ was about 10 percent greater than the estimated value $\left(6.17 \times 10^{-6} \mathrm{~cm}^{2} / \mathrm{sec}\right)$. The stokes-Einstein radius of TPP obtained from the experimental bulk diffusion coefficient was $7.24 \AA$.

The results of the hindered diffusion experiments of TPP are presented in Table 6.13, where the average membrane pore sizes, the measured effective diffusivities, the ratios of the stokes-Einstein radii to the pore sizes of membranes, 
154

Table ,.12

Measurements of the Bulk Diffusion Coefficient of TPP in Ethyl Acetate $25^{\circ} \mathrm{C}$.

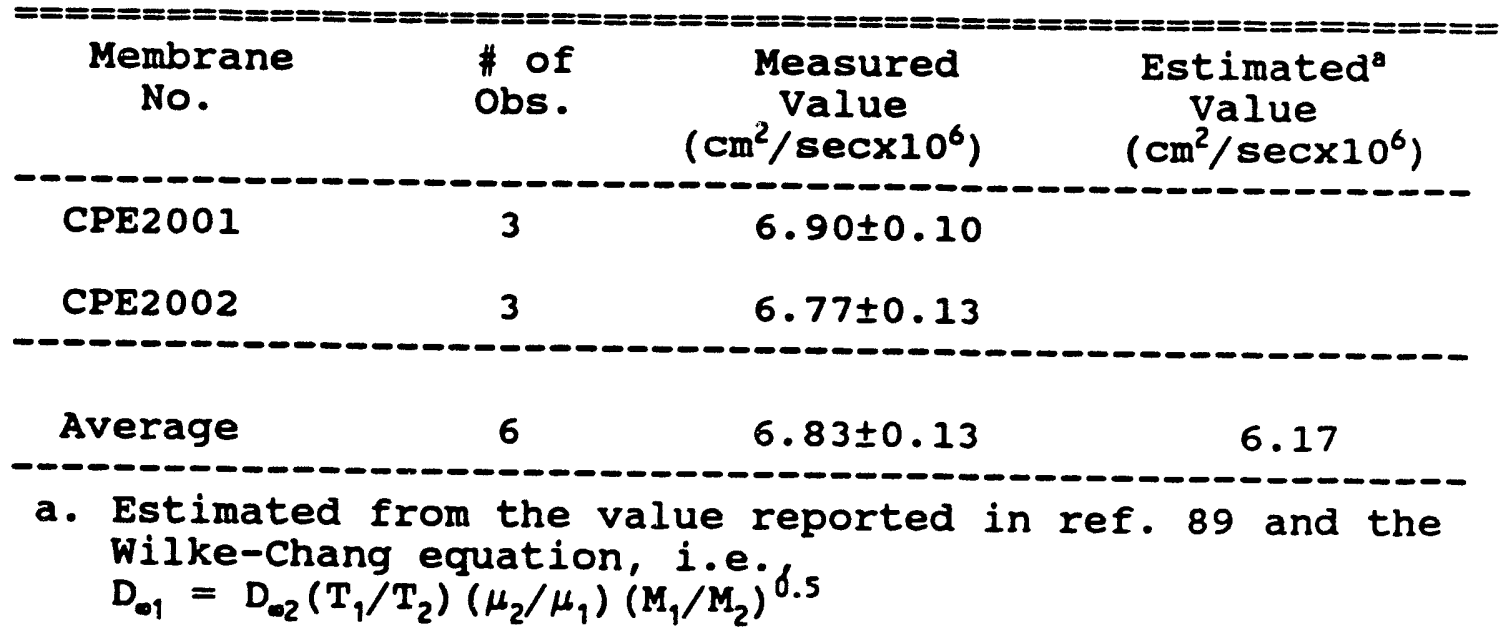


Table 6.13

The Results of the Hindered Diffusion Experiments of TPP in Ethyl Acetate e $25^{\circ} \mathrm{C}$.

\begin{tabular}{|c|c|c|c|c|}
\hline $\begin{array}{c}\text { Membrane } \\
\text { No. }\end{array}$ & $\begin{array}{l}\text { Avg } \\
\text { Pore Dia. } \\
\text { (A) }\end{array}$ & $\begin{array}{c}\text { Effective } \\
\text { Diffusivity } \\
\left(\mathrm{cm}^{2} / \sec ^{\circ} 10^{6}\right)\end{array}$ & $r_{s} / r_{0}$ & $D_{e f f} / D_{0}$ \\
\hline $\begin{array}{l}\text { TPE0451 } \\
\text { TPE0452 } \\
\text { TPE0601 } \\
\text { TPE0602 } \\
\text { TPE0602 } \\
\text { TPE0603 } \\
\text { TPE0604 } \\
\text { TPE0901 } \\
\text { TPE0902 } \\
\text { TPE0903 } \\
\text { TPE0903 } \\
\text { TPE1201 } \\
\text { TPE1202 } \\
\text { TPE1202 } \\
\text { TPE1501 } \\
\text { TPE1501 } \\
\text { TPE1503 } \\
\text { TPE1503 } \\
\text { TPE2401 } \\
\text { TPE2401 } \\
\text { TPE2402 }\end{array}$ & $\begin{array}{r}75.1 \\
77.5 \\
89.4 \\
101.6 \\
101.6 \\
105.3 \\
110.4 \\
123.2 \\
138.7 \\
144.2 \\
144.2 \\
198.8 \\
224.8 \\
224.8 \\
252.2 \\
252.2 \\
272.2 \\
272.2 \\
451.5 \\
451.5 \\
467.8\end{array}$ & $\begin{array}{l}1.90 \\
2.64 \\
3.67 \\
3.22 \\
3.61 \\
3.87 \\
3.76 \\
3.86 \\
4.56 \\
4.67 \\
4.13 \\
3.65 \\
5.67 \\
5.80 \\
5.56 \\
5.08 \\
5.54 \\
5.76 \\
6.42 \\
6.40 \\
6.30\end{array}$ & $\begin{array}{l}0.193 \\
0.187 \\
0.162 \\
0.143 \\
0.143 \\
0.138 \\
0.131 \\
0.116 \\
0.104 \\
0.100 \\
0.100 \\
0.073 \\
0.064 \\
0.064 \\
0.057 \\
0.057 \\
0.053 \\
0.053 \\
0.032 \\
0.032 \\
0.031\end{array}$ & $\begin{array}{l}0.278 \\
0.386 \\
0.537 \\
0.472 \\
0.529 \\
0.567 \\
0.551 \\
0.565 \\
0.668 \\
0.684 \\
0.606 \\
0.534 \\
0.850 \\
0.830 \\
0.817 \\
0.744 \\
0.811 \\
0.844 \\
0.941 \\
0.937 \\
0.922\end{array}$ \\
\hline
\end{tabular}

a. Calculated from Equation 2.2 negelecting boundary layer resistances and entrance-exit effect

b. Pore diameters were measured using ternary multicomponent diffusion experiments (TPP and n-octane in the same diffusion cell). 
and the ratios of the effective diffusivity to the bulk diffusivity are listed. As shown in this table, the effective diffusivity of TPP increased as the average pore sizes of membrane increased and the effective diffusivity data of several duplicate runs are reproducible within $5.2 \mathrm{X}$ $10^{-7} \mathrm{~cm}^{2} / \mathrm{s}$. The results are also presented in graph form in Figure 6.5 where $D_{e} / D_{0}$ is plotted against $r_{s} / r_{0}(\lambda)$. The solid line in this curve is drawn from the theory proposed by Anderson et al. (89) for the hindered diffusion of thin disk-shaped molecules. The equation is expressed as:

$$
\frac{D_{\theta}}{D_{\infty}}=\left(1-\left(\frac{\pi^{2}}{8}\right) \lambda\right)^{2}\left(1-2.1044 \lambda+2.088 \lambda^{3}-0.948 \lambda^{5}\right) \quad(6.3)
$$

One noticeable aspect of this equation was that two length scales of a molecule (the mean projected length used in partitioning term and the stokes-Einstein radius used in mobility correction term) were used to describe the molecular size and shape. This approach (the use of two length scales for the nonspherical molecules to describe the characteristic length of molecules) has been used successfully in several recent hindered diffusion studies to explain the experimental results $(47,99,105)$.

As shown in Figure 6.5, it appears that the hindered diffusion data fall slightly above this theoretical curve. To clarify this observation, several statistical methods were used to examine the significance of the difference between the experimental data and the theoretical curve. 


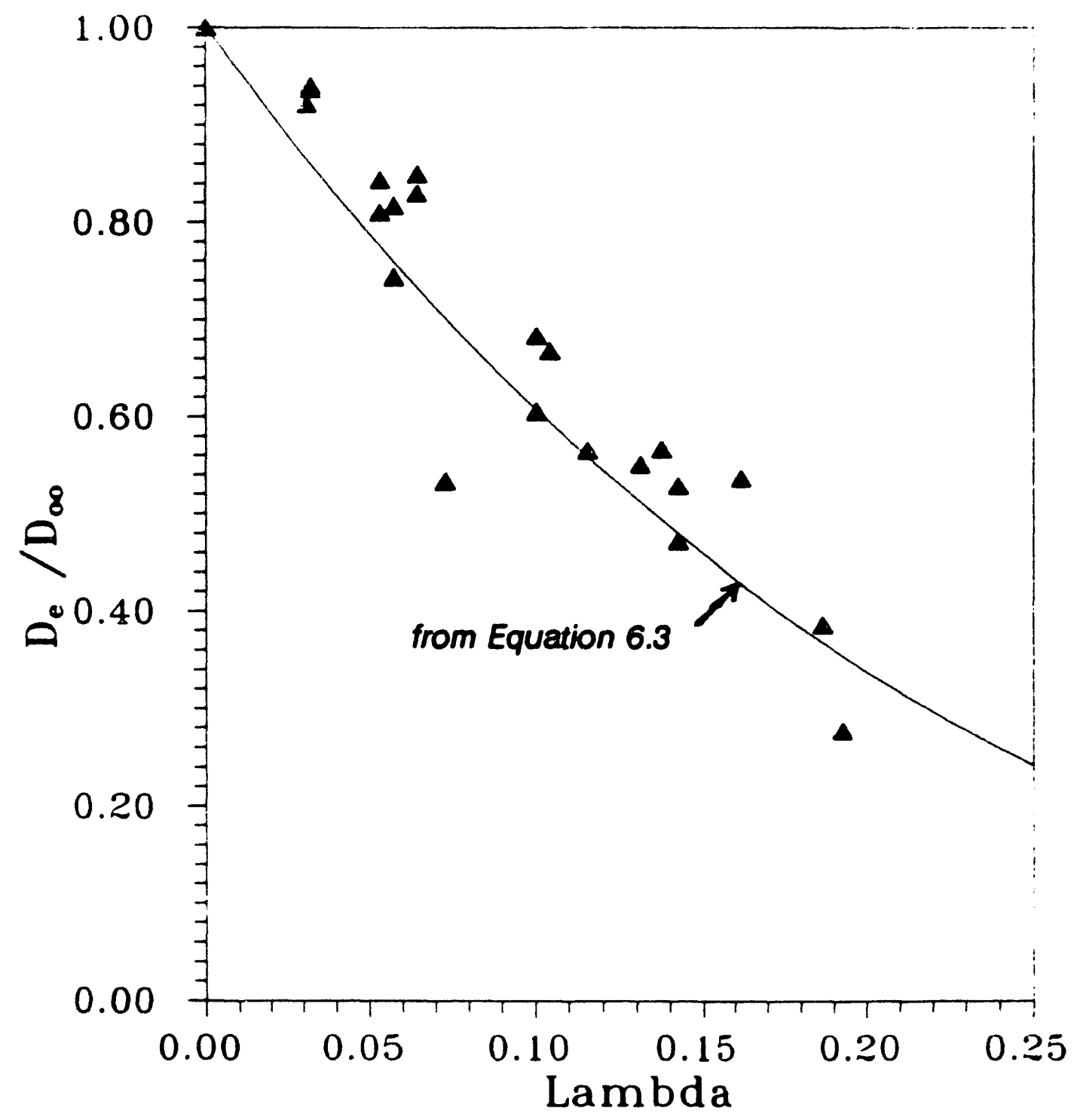

Figure 6.5 $\begin{aligned} & \text { Dependence of } D_{e} \\ & \text { Diffusion of TPP }\end{aligned} / D_{0}$ on $\lambda$ in hindered 
The results of the statistical studies are summarized in Table 6.14.

Two nonlinear curve-fitting methods in the table had been used in several previous hindered diffusion studies $(54,56,29)$, whereas the other curve-fitting method was a second degree polynomial regression. For each curve-fitting method, the parameters of the theoretical curve were obtained by using 300 theoretical data points ranging from 0.0 to 0.3 calculated from Equation 6.3 . As shown in this table, the parameter from the theoretical data is outside the $99 \%$ confidence level limits of the model parameters for the two nonlinear fittings from the experimental data. Differences between theoretical and experimental data also can be seen in the second degree polynomial regression. Probabilities of hypothetical tests for closeness between the theoretical and experimental polynomial coefficients are almost zero.

Results obtained from this study are also different from the findings of the hindered diffusion study of TPP with chloroform using a track-etched mica membrane reported by Anderson et al. (89). Their experimental data were in excellent agreement with the theoretical predictions. Here, any possible experimental reasons or theoretical reasons were investigated in order to explain the differences between the theoretical curve and the experimental data in the current study. 


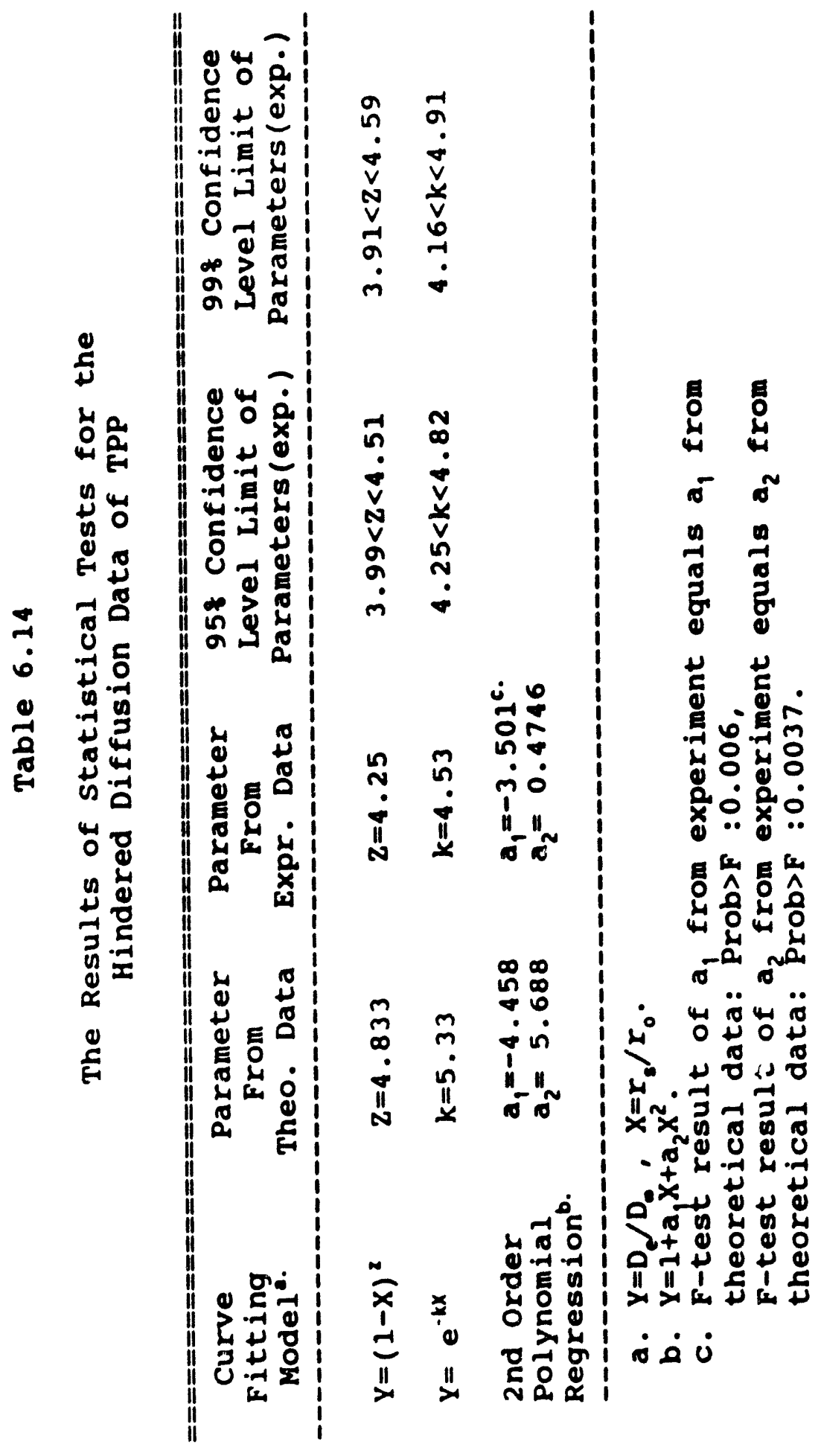


The possible experimental errors can be classified into two groups: those arising during the diffusion experiments themselves, and those arising during the analysis of experimental data, such as errors in instrumental analysis or errors introduced by assumptions in the equations used in the analysis of experimental data.

Since most of the current experimental data points fall above the theoretical curve, the most suspicious errors introduced during the experiments are those caused by convective flows.

The convective flow can occur for several reasons, such as temperature variations in the water bath, pressure fluctuation caused by rotation of the stirrer, pressure differences between the two compartments of the diffusion cell, or hydrostatic pressure caused by leaks.

The temperature variation of the water bath used in the current study was less than $0.02{ }^{\circ} \mathrm{K}$, which caused only about $3.68 \times 10^{-6} \mathrm{~cm}^{3}$ of expansion and contraction of ethyl acetate. Such an expansion and contraction could produce a negligible amount of convective flow during the experiments, which usually lasted more than 24 hours, if the temperature variation cycle was longer than 2 minute (less than $1 \%$ of the diffusion rate). Because of the large reservoir of the water bath used in the current study, this frequent temperature variation cycle would not occur. Thus, the errors caused by temperature variation can be dismissed. 
The rest of the error sources for the convective flow can only be significant when the diffusion cell is used in an open system. Since the diffusion cell used in the current study was a batch-type closed system and was placed in the water bath during the experiment, any significant pressure disturbance across the two compartments of the diffusion cell could not be produced.

other experimental error sources, such as osmotic flow effect or possible TPP degradation during the experiment, would have forced the measured data points to move in the opposite direction (below the theoretical curve). Thus, these kinds of errors were not considered here.

Likewise, change in the pore size during the experiments due to the adsorption of solute was not regarded as a possible reason since it would cause the same effect. Actually, the pore radii calculated from diffusivity measurements of $n$-octane in ethyl acetate before and after the diffusion experiments did not differ by more than $3 z$, as shown in Table 6.15 .

Next, discussion will center on errors arising during the analysis of experimental data. The most likely sources of potential error in the analysis of experimental data come from the assumptions used in the membrane characterization process. In this hindered diffusion experiment of TPP, the pore densities of the membranes were not actually measured. Instead, the pore density specified 
Table 6.15.

Changes in Calculated Pore Diameter of Polyester Membranes During the Hindered Diffusion Experiments of TPP

\begin{tabular}{|c|c|c|}
\hline Membrane No. & $\begin{array}{c}\text { Calculated } \\
\text { Before } \\
\text { TPP Diffusion }\end{array}$ & $\begin{array}{c}\text { Pore Size (A) } \\
\text { After } \\
\text { TPP Diffusion }\end{array}$ \\
\hline TPE0605 & 105.3 & 108.7 \\
\hline TPE0606 & 110.4 & 111.1 \\
\hline
\end{tabular}


by the manufacturer was used in the calculation to analyze the experimental data as previously discussed in chapter III.

Because of the assumptions used in the calculation, a sensitivity analysis of the variation in pore densities to the experimental data points shown in Figure 6.5 was conducted. Details of this study are reported in Appendix F. One striking result of this study was that increasing or decreasing the pore densities only made the experimental data points moved along the theoretical curve. In other words, changes in pore densities did not cause the experimental data points to move above or below the theoretical curve. Also, the changes in data points were not sensitive to the variations in pore densities of membranes. Thus, it could be concluded that the observation seen in Figure 6.5 was not caused by errors introduced by using nominal pore densities in the calculation.

Errors introduced by instrumental analysis can also be neglected, since the measurement errors in the UV analysis are usually less than $1.0 \%$.

In the light of the experimental reasons explaining the differences between the experimental data and theoretical curve, it was concluded that any experimental errors would not cause the observation seen in Figure 6.5 .

Next, the discussion will focus on the theoretical aspects of hindered diffusion. As previously noted, the 
reduction in diffusivity of solute in the hindered diffusion experiment is due to two factors: the partitioning effect and the frictional effec... The first bracket of Equation 6.3 represents the partitioning effect term, while the second bracket represents the frictional effect term. The expression for the frictional effect in Equation $6.3 \mathrm{had}$ been derived for the movement of a rigid spherical solute in a cylinder by using the "centerline approximation" (the sphere on the cylinder's centerline). Since the molecule used in the current study was a rigid disk-shaped molecule, this zpproximation might produce an error in the theoretical curve. However, the approximate expression used in Equation 6.3 has been used successfully in many previous hindered diffusion studies by implementing the hydrodynamic radius calculated from the stokes-Einstein equation. Also, use of the centerline approximation underestimated the frictional factor. Thus, it appeared that the differences observed in Figure 6.5 were not caused by using the approximate equation derived for the hard sphere.

A more rigorous treatment concerning the mobility factor of this molecule in a cylindrical pore might be required to evaluate the experimental results. However, this kind of approach was not available in the literature to date.

Compared to the frictional factor, the partition coefficient term has been studied quite extensively for the 
various shapes of molecules in differently shaped pores $(41,103,100)$. The partition coefficient term is the equilibrium ratio of pore to bulk concentration of solute. The general expression for the partition coefficient of arbitrary shape of molecule in an arbitrary pore is:

$$
K_{p}=\frac{\iiint \exp \left(-E_{p}(\zeta, v, \xi)\right) d \zeta d v d \xi}{\iiint \exp \left(-E_{b}(\xi)\right) d \zeta d v d \xi}
$$

Here $\zeta, v$, and $\xi$ are the general coordinates describing molecular position, orientation and conformation, respectively. $E_{p}$ and $E_{b}$ are the interaction potentials of the molecule in the pore and bulk. This equation can be written in a more simplified form with the following considerations: (1) the solute is a rigid molecule, i.e., only a single conformation exists; and (2) the solution is infinitely dilute, and thus is ideal. Equation 6.4 becomes:

$$
K_{p}=\frac{\iint \exp (-E(\zeta, v)) d \zeta d v}{\iint d \zeta d v}
$$

Here, $\mathrm{E}$ is the interaction potential between the solute molecule and the pore wall. The physical interactions between the solute and pore wall are usually the result of the attractive or repulsive van der waals forces or repulsive electrostatic interactions $(E<0)$. As previously discussed, if $E=0$, then the partition coefficient represents only the steric effect. For the sphere in a cylindrical 
pore with pure steric interaction, Equation 6.5 becomes:

$$
K_{p}=\left(1-\frac{a}{r_{0}}\right)^{2}
$$

For the spherical solute, the radius of the molecule, 'a', is the same as the stokes-Einstein radius. However, for the rigid disk-shaped molecule, another molecular length scale representing the orientation and size should be used as shown in Equation 6.5 .

Giddings et al.(41) found that the use of the mean external (projected) length to describe the partitioning behavior of nonspherical molecules was better than the use of any other measure of molecular size. The mean projected length for the axisymmetric molecule is defined as:

$$
X_{m}=\int_{0}^{\frac{2}{x}} h(\theta) \sin \theta d \theta
$$

where $\mathrm{h}$ is the closest approach length of the center of the molecule and $\theta$ is the angle between the axis of the molecule's rotation and the unit normal vector of molecules.

Baltus (103) reported the following expression relating the stokes-Einstein radius to the mean projected length for disk-shaped molecules by combining Gidding's expression for the mean projected length and Happel and Brenner's work (120) for the effect of the hydrodynamic radius of the diskshaped molecule.

$$
\frac{X_{m}}{I_{s}}=\frac{\sin ^{-1}\left(1-\phi^{2}\right)^{0.5}}{\left(1-\phi^{2}\right)^{0.5}}\left\{\frac{\phi}{2}+\frac{\sin ^{-1}\left(1-\phi^{2}\right)^{0.5}}{2\left(1-\phi^{2}\right)^{0.5}}\right]
$$


Here, $\phi$ is the ratio of minor-to-major axis. As the value of $\rightarrow$ approaches zero, the value of $\mathrm{x}_{\mathrm{m}} / \mathrm{r}_{\mathrm{s}}$ in Equation 6.8 beccmes the asymptotic value, $\pi^{2} / 8$. The expression in the first bracket of Equation 6.3 was obtained from the aforementioned process.

Since TPP has a certain thickness because of $\pi$ electrons, the $\phi$ value is greater than zero. In Figure 6.6, the theoretical curves with different $\phi$ values are presented along with the TPP hindered diffusion experimental data. As shown in this figure, the introduction of the $\phi$ parameter in the theoretical curve still does not explain the differences between the experimental data and the theoretical curve.

The only term which was not considered up to thi:s point was the interaction between solutes and pore walls. The use of Equation 6.3 assumes that there are no solute-pore wall interactions. However, if the attractive interaction energy is introduced to the theoretical curve as shown in Equation 6.5 , the partition coefficient increases, and the theoretical curve for $D_{e} / D_{0}$ thus moves in the experimental data points' direction (upward).

As Deen (39) pointed out in his review paper on hindered diffusion, few studies have been conducted previously concerning the characterization of solute-pore wall interactions, especially attractive forces. However, a simulation study concerning the partitioning of a polymer with attractive interactions conducted by Davidson et al. 


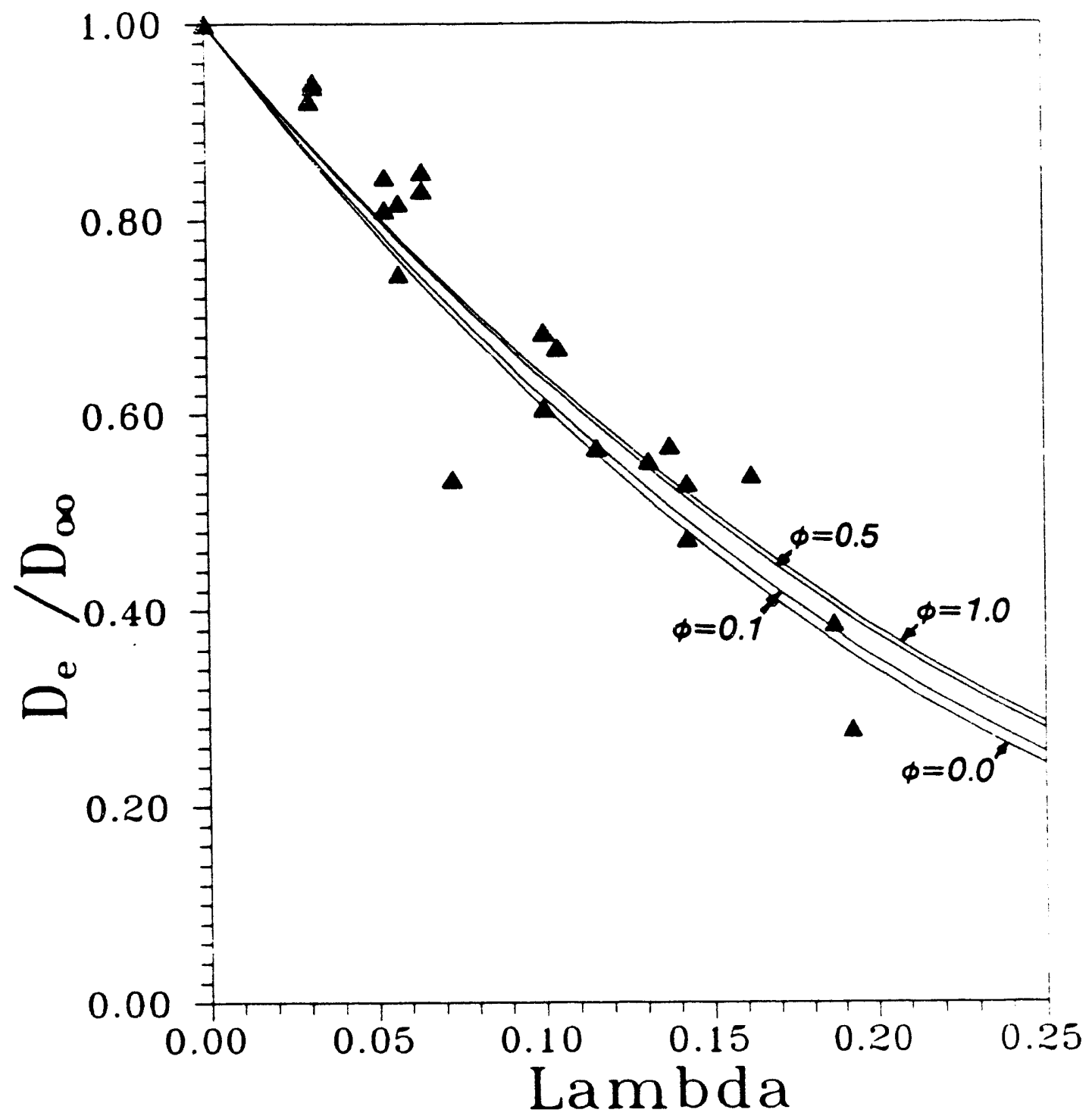

Figure 6.6. Changes in Theoretical Curve and The Hindered Diffusion Data of TPP 
(100) using a Monte Carlo simulation technique showed that the partition coefficient increased substantially when moderate attraction energy was included.

strong attractive interactions might eventually lead to the adsorption of solutes on the pore wall. However, as seen in Table 6.15, the pore diameters were not changed during the experiment, which is an evidence that the adsorption of TPP had not occurred in the current experiments. It should be noted that adsorption of solute will occur only if the interaction energy is above some threshold value. For a positive value of interaction energy below the threshold value, the solute is merely attracted toward the surface of the pore wall. Since our experimental data fell slightly above the theoretical curve, the result indicated that weak interactions occurred between TPP and the pore walls of polyester membranes.

In comparing the current experimental results with the TPP hindered diffusion experimental results conducted by Anderson et al. (89), their experimental data almost fell on the theoretical curve; thus, the concept of attractive interaction was not required to evaluate their experimental data. The solute-pore wall interactions are the result of complex interactions: between solute and pore wall, between solvent and pore wall, and between solute and solvent. since their experimental system was different from that used in the current study (e.g., track-etched mica membrane 
/polyester membrane, chloroform/ethyl acetate), TPP might experience different interactions in the current study. One thing to be noted in their experiment is that the shape of mica membrane pore opening was rhombohedral. This fact leads to some ambiguity in defining an "equivalent" circular pore, since theoretical equations are not available for rhombohedral pores. 


\section{DIFFUSION OF COAL MACROMOLECULES}

As noted in Chapter $I$, the objective of the present research is to measure the diffusion coefficients of coal macromolecules and to determine the dependence of the effective diffusivity on the ratio of molecule to pore size. Diffusion experiments of coal macromolecules were conducted to accomplish this objective. The experiments and analyses conducted for this study were based on the results of the model compounds' diffusion studies.

Coal macromolecules used in the current study were prepared from a sample obtained from the coal liquefaction pilot plant of Southern Electric International, Inc. (Wilsonville, Alabama). The sample was collected from the deashed residue stream of the ROSE_SR ${ }^{S M}$ unit at the Wilsonville coal liquefaction plant, which was running Illinois No. 6 coal. Most of this collected sample was dissolved in ethyl acetate (about 97\%) at a low concentration (about $300 \mathrm{mg} / \mathrm{l}$ ).

Since coal asphaltene is a complex mix ure of molecules having different shapes and sizes, the measurements of effective diffusion coefficients of coal macromolecules were not as simple as the diffusivity measurement of model compounds. 
In the current study, gel permeation chromatography (GPC) was used to circumvent this difficulty. In GPC analysis, materials are separated on the basis of their hydrodynamic volumes. By the use of GPC, coal asphaltene can be separated into several fractions with relatively narrow size distributions. This allows the diffusion coefficients of each fraction to be analyzed separately using the results of the diffusion experiment. Baltus et al. (51) used this technique in their diffusion coefficient measurements of petroleum asphaltene. As noted in the literature $(69,90)$, coal asphaltene is different from petroleum asphaltene in many respects, particularly in terms of its size and constituents. Coal asphaltene is much smaller than petroleum asphaltene. Thus, the results of petroleum asphaltene diffusion were not directly applicable to our diffusion study of coal macromolecules.

In this research, GPC calibration was conducted first in order to characterize the coal macromolecules. Next, the effective diffusivity measurements of coal macromolecules were performed.

\section{GPC Calibration}

GPC analysis of coal macromolecules requires the use of calibration standards to determine the average molecular weights of its fractions from elution volumes.

Several studies reported in the literature discussed the selection of calibration standards in the GPC analysis 
173

of coal liquids (91-97), and it was generally agreed that the calibration curve obtained from the polystyrene standards gave a reasonable approximate value of the molecular weights of coal asphaltene fractions compared to the values measured from other methods, such as vapor pressure osmometry $(92,97)$. Thus, the calibration curve was first obtained by the use of polystyrenes and polycyclic aromatic hydrocarbons as calibration standards, and a sample of the coal molecule solution was analyzed.

\section{Experiments}

Polystyrene standards used in this experiment were obtained from Pressure Chemical (Pittsburgh, PA). The physical properties provided by the manufacturer are given in Table 7.1 .

Five polycyclic aromatic hydrocarbons (with molecular weights ranging from 128 (naphthalene) to 523 (rubrene)) and tetraphenylporphine were purchased from Aldrich Chemical Co. The molecular weights and chemical structural formulas of these compounds are presented in Table 7.2 .

The gel permeation chromatography used in this experiment consists of a solvent delivery pump (Waters Associates, Model 590), sample injector (Waters, Model U6K), variable wave length U.V. detector (Waters, Model 480), and GPC column banks with column heater (Waters, Model RCM-100) and temperature control module (Waters). Two Ultrastyragel columns (Waters) with average pore diameters of $500 \AA$ and 
Table 7.1

Molecular Weights of Polystyrenes and GPC $^{*}$ Elution Volumes

\begin{tabular}{|c|c|c|c|c|c|c|}
\hline Lot No. & 25169 & 50123 & 31116 & 40627 & 31019 & 11103 \\
\hline $\begin{array}{l}\text { Weight } \\
\text { Average } \\
\text { M.Wt (Mw) }\end{array}$ & 3100 & 2276 & 1574 & 1306 & 1152 & 800 \\
\hline $\begin{array}{l}\text { M. wt } \\
\text { Distrib. } \\
(\mathrm{Mw} / \mathrm{Mn})\end{array}$ & 1.29 & 1.06 & 1.01 & 1.07 & 1.07 & $<1.2$ \\
\hline $\begin{array}{l}\text { Peak Elution } \\
\text { Volume (ml) }\end{array}$ & 23.38 & 23.98 & 25.18 & 25.71 & 26.04 & 28.07 \\
\hline
\end{tabular}


Table 7.2

Polycyclic Aromatic Hydrocarbons and Porphine used as GPC standards and GPC Operating Conditions.

\begin{tabular}{|c|c|c|c|c|c|}
\hline Name & M.Wt & $\begin{array}{l}\text { Chemical } \\
\text { struct' } 1 \\
\text { formula }\end{array}$ & $\begin{array}{c}\text { Peak } \\
\text { elution } \\
\text { volume (ml) }\end{array}$ & $\begin{array}{l}\mathrm{U} \cdot \mathrm{V} \text {. } \\
\text { detector } \\
\text { length }(\mathrm{nm})\end{array}$ & $\begin{array}{l}\text { Sample } \\
\text { conc. } \\
(g / 1)\end{array}$ \\
\hline $\begin{array}{l}\text { Naphtha- } \\
\text { lene }\end{array}$ & 128.2 & & 40.04 & 268 & .532 \\
\hline $\begin{array}{l}\text { Anthra- } \\
\text { cene }\end{array}$ & 178.2 & & 39.29 & 276.6 & .443 \\
\hline $\begin{array}{l}\text { Triphen- } \\
\text { lene }\end{array}$ & 228.3 & & 39.07 & 258.4 & .0355 \\
\hline $\begin{array}{c}\text { Coro- } \\
\text { nene }\end{array}$ & 300.4 & & 41.65 & 302.8 & .0355 \\
\hline Rubrene & 532.7 & & 32.76 & 300.1 & .0177 \\
\hline$T P P$ & 614.8 & & 32.07 & 431.2 & .0035 \\
\hline
\end{tabular}


$100 \AA$ and two $5 \mu$-Phenogel columns (Pheriomax) with average pore diameters of $100 \AA$ and $50 \AA$ were connected in a series (order: $500 \AA-100 \AA$ (Water) - $100 \AA$ (Phenomax) - $50 \AA$ ) in the column heater. The temperature of the column heater was controlled at $50{ }^{\circ} \mathrm{C}$ to minimize the possible interactions of standard compounds with column packing material.

Tetrahydrofuran (THF) was used as a mobile phase since it also minimized the possible interactions of compounds and coal macromolecules with GPC column packing material compared to other solvents. THF (HPLC grade) was obtained from Fisher scientific Co. Before THF was used in GPC analysis, it was filtered through a Nylon membrane (Supleco, Bellefonte, PA) having $0.2 \mu \mathrm{m}$ nominal pore size and was degassed for 2 mirutes by the use of a sonicator under a vacuum. The THF reservoir was sparged with He gas to keep THF from forming peroxides. Peroxides provide a background absorbance on a UV detector and make the baseline unstable. A relatively stable baseline was obtained in the current study by keeping the He gas supply pressure higher than 10 psig. The flow rate of $\mathrm{THF}$ was set at $1 \mathrm{ml} / \mathrm{min}$. Other operating parameters for calibration standard compounds are also summarized in Tables 7.1 and 7.2 .

A solution of coal macromolecules for the GPC analysis was prepared as follows. A $0.3 \mathrm{~g}$ of the original sample was dissolved in $1000 \mathrm{ml}$ of degassed ethyl acetate (optima grade, Fisher Scientific). This solution was then filtered 
through a Nylon membrane (Supelco) having a $0.2 \mu \mathrm{m}$ pore diameter. About 2.5 of the original "jample was not dissolved in ethyl acetate and was left on the membrane filter after filtration. Also, a small amount of ethyl acetate was evaporated during the filtration step. Thus, the final concentration of the prepared solution (called the stock solution hereafter) was abont $299 \mathrm{mg} / \mathrm{l}$. About $50 \mathrm{\mu l}$ of the stock solution was injected to GFC to characterize the coal macromolecules. UV absorbance of the solution was measured at $263 \mathrm{~nm}$.

\section{Results and Discussion}

Logarithms of the number of average molecular weights of polystyrenes and logarithms of molecular weights for other standard compounds are plotted against the elution volume of each compound in Figure 7.1. As shown in this figure, a typical sigmoid shape GPC curve was obtained. This figure indicates that the compounds with an elution volume of approximately less than $24 \mathrm{ml}$ are excluded, and the compounds with an elution volume roughly greater than 39 ml are permeated so that no further separation is possible with the current column setting. The linear region in Figure 7.1 reasonably covers the molecular weight range of coal asphaltene.

Baltle et al. reported (92) that the calibration curve of GPC constructed from polystyrenes and polycyclic aromatic 


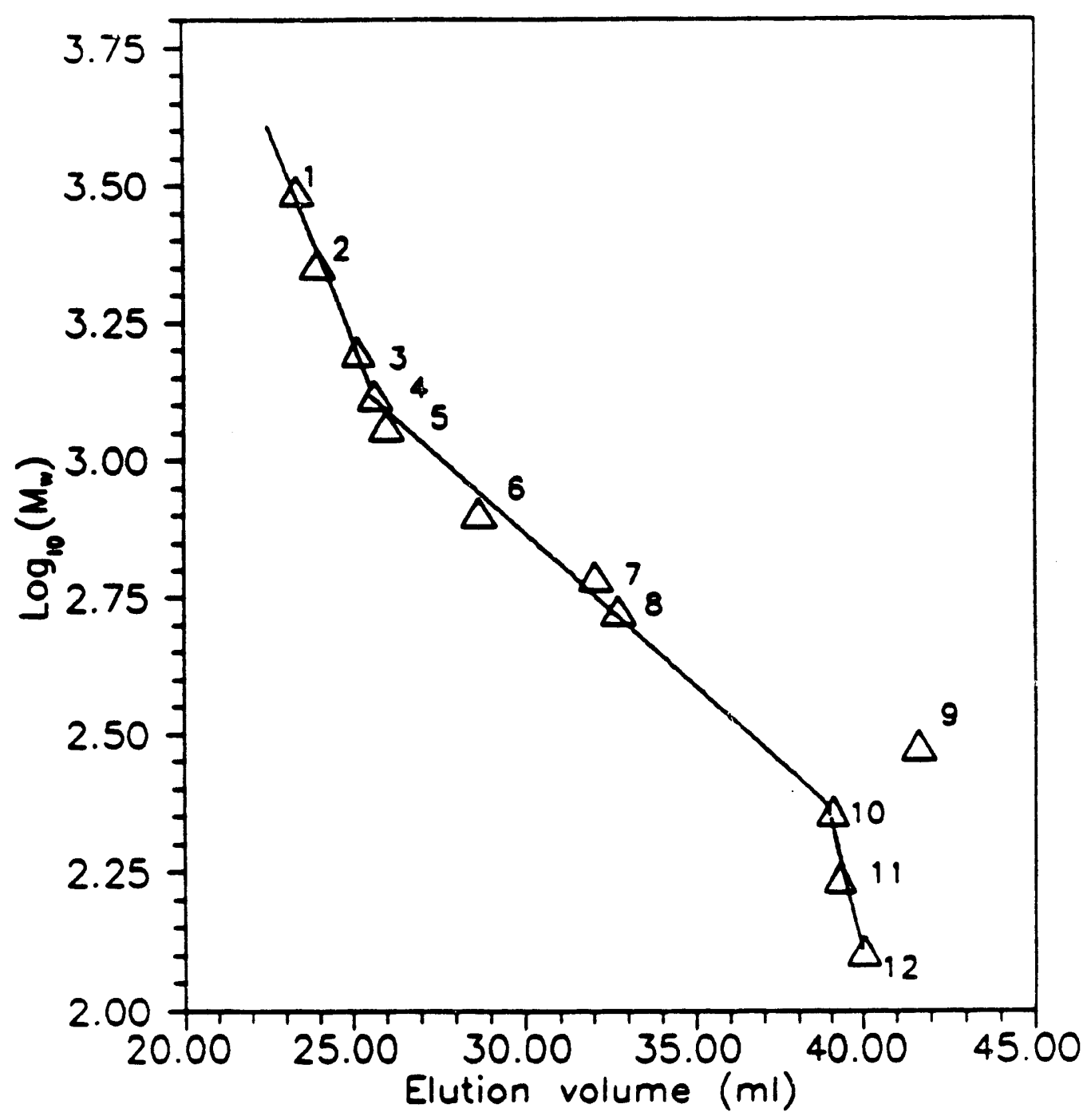

Figure ?.1 GPC Calibration Curve for Coal Asphaltene (1.P/S Mw 3100, 2.P/S MW 2276, 3.P/S MW1574, 4.P/S Mw 1306, 5.P/S MW 1152, 6.P/S MW 800, 7.TPP, 8.Rubrene, 9.Coronene, 10.Triphenylene, 11. Anthracene, 12. Naphthalene) 
hydrocarbons agreed relatively well with the curve obtained from the logarithms of actual molecular weights of coal asphaltene fractions and their GPC elution volumes, especially for the fractions having average molecular weights of less than 1000. In their study, the actual molecular weight of coal asphaltene was obtained from the preparative GPC and the vapor pressure osmometry. Thus, it was expected that the calibration curve shown in Figure 7.1 could predict the molecular weight of coal molecules.

In Figure 7.1 , coronene shows a higher elution volume than that would be expected from its molecular size. This Lnusual behavior of pericondensed polycyclic aromatic hydrocarbons in GPC analysis has been reported in the literature $(92,98)$. These observations were attributed to the susceptibility of adsorption to the column packing material for pericondensed polycyclic aromatic hydrocarbons such as roronene, pyrene and perylene.

The slope and intercept of the linear line in Figure 8.1 gave a correlation equation expressed as:

$$
\log _{10} M_{w}=-0.56 V_{i}+4.55
$$

Where, $M_{w}=$ Molecular weight and $V_{i}=$ Elution volume (ml). Figure 7.2 shows a chromatogram of the stock solution of coal macromolecules obtained from GPC analysis. In this figure, the calibration standards equivalent molecular weights for the asphaltene components are presented along the abscissa of the elution volume. The chromatogram shows 
180

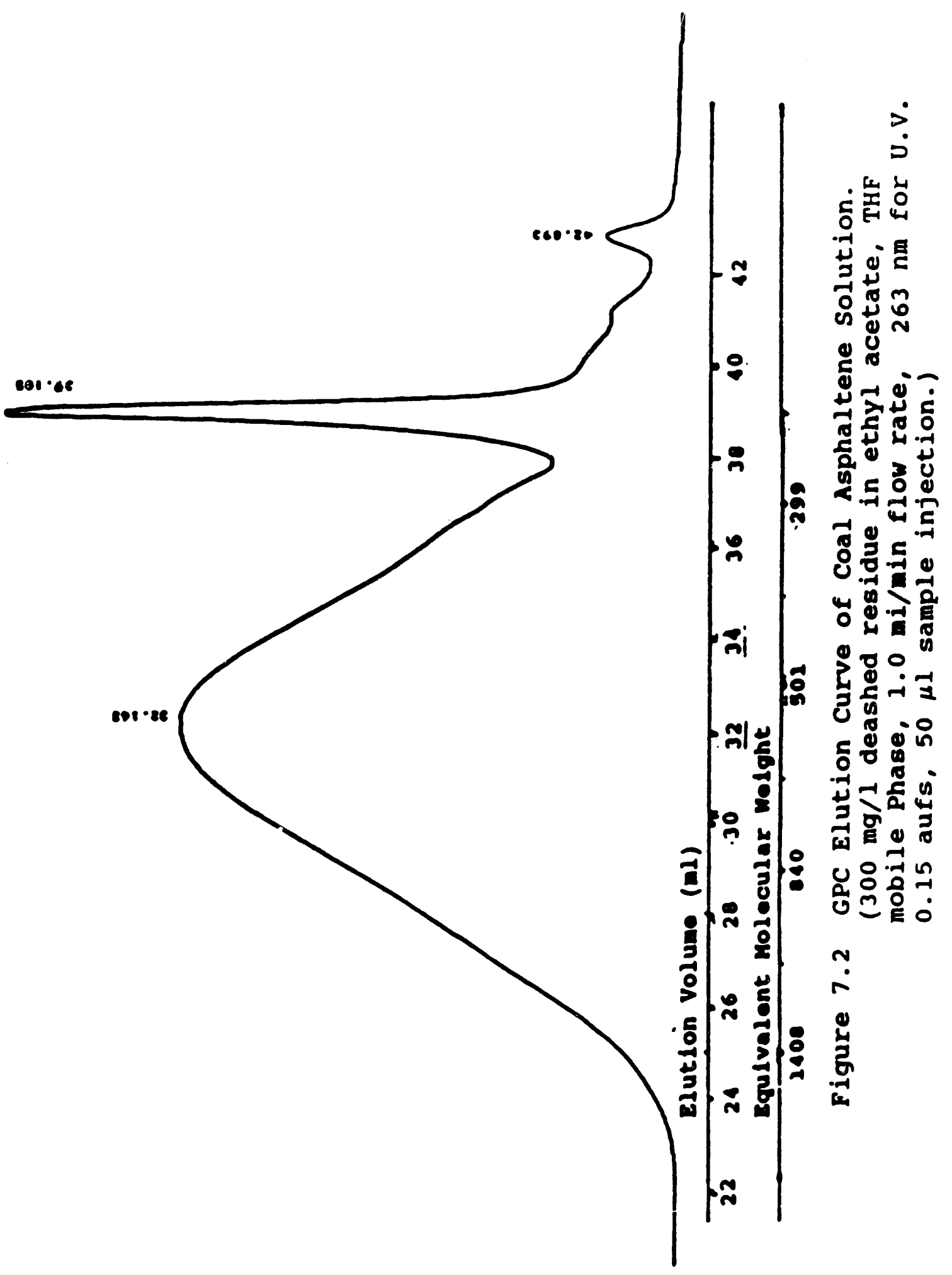


181

that the molecular weights of the stock solution range from 300 to 1500. The major fraction of coal macromolecules used in the study appeared to be asphaltene. The chromatogram also shows an interesting shape. As shown in Figure 7.2, there were no significant elution tails in this chromatogram, and the curve was almost a bell shape. This indicated that the interactions between coal molecules in the sample solution and column packing material were not significant. The sharp peak around the elution volume of 39 $\mathrm{ml}$ is the ethyl acetate peak.

\section{Effective Diffusion Coefficient Measurements of Coal Macromolecules}

The effective diffusivities of coal macromolecules were measured in this last part of the current study. Also, all experimental data obtained from the diffusion experiments were analyzed on the basis of the hindered diffusion theory.

\section{Experiments}

Diffusion experiments were conducted with polyester membranes prepared in the same manner as described previously in the hindered diffusion experiment of TPP. The pore sizes of these membranes were determined by in situdiffusivity measurements of n-octane because there was an evidence that adsorption of coal molecules to pore walls occurred during the diffusion experiments. Details related to these measurements will be described later. 
The stock solution of coal macromolecules used in these diffusion experiments was identical tc that prepared for the GPC analysis. Experimental procedures were simjilar to those previously used, except that the premixed stock solution was directly charged in one compartment of the diffusion cell. The solubility of coal macromolecules in ethyl acetate was not high enough to prepare a concentrated solution which would allow the use of the syringe injection technique used in the previous model compound diffusion experiments. In these experiments, the diffusion cell, the stock solution and degassed ethyl acetate were kept in an air-circulating chamber maintained at $25^{\circ} \mathrm{C}$ long enough to reach a thermal equilibrium. The upper compartment of the diffusion cell was then filled with ethyl acetate and the bottom compartment was charged with the stock solution. Since most of the hindered diffusion experiments of coal macromolecules lasted more than 24 hours at least, any concentration changes in the diffusion cell that occurred during this relatively short charging time (usually less than $3 \mathrm{~min}$.) could be neglected.

As previously noted, the pore sizes of membranes were determined from the in situ-diffusivity measurements of $n$ octane during the hindered diffusion experiments of coal macromolecules. With this technigue, n-octane was introduced into the diffusion cell as a tracer (usually less than 0.5 wt? of the solution in one compartment) during the 
coal diffusion experiments. A time for the introduction of n-octane to the diffusion cell was carefully selected to ensure that a proper amount of n-octane had passed through the membrane pores by the time the diffusion experiment was finished, since the diffusivity of octane was much higher than that of the coal molecules.

In the hindered diffusion study of petroleum asphaltene, Baltus (121) reported that the pore diameters of the mica membranes measured before and after the diffusion experiments of petroleum asphaltene usually decreased by more than 10\%. A detailed explanation concerning this observation was not given in her work. However, it appeared that adsorption of petroleum molecules having high functional groups might cause this phenomena. According to Yen's work (90), coal asphaltene also had many functional groups. Thus, it was suspected that the adsorption phenomena would occur in the current experiments. A separate study to investigate the changes in pore diameter of membranes was conducted. In these experiments, the initial pore diameters of membranes were determined by measuring the diffusivity of n-octane in pure ethyl acetate. Next, the changes of pore diameter were observed during two consecutive diffusion experiments of coal molecules. Final pore diameters of membranes were measured by conducting the n-octane diffusion experiment again with pure ethyl acetate. The results are presented in Table 7.3. As shown in this 


\section{4}

Table 7.3

Changes in Calculated Pore Diameter of Membranes Used in Hindered Diffusion of Coal Macromolecules

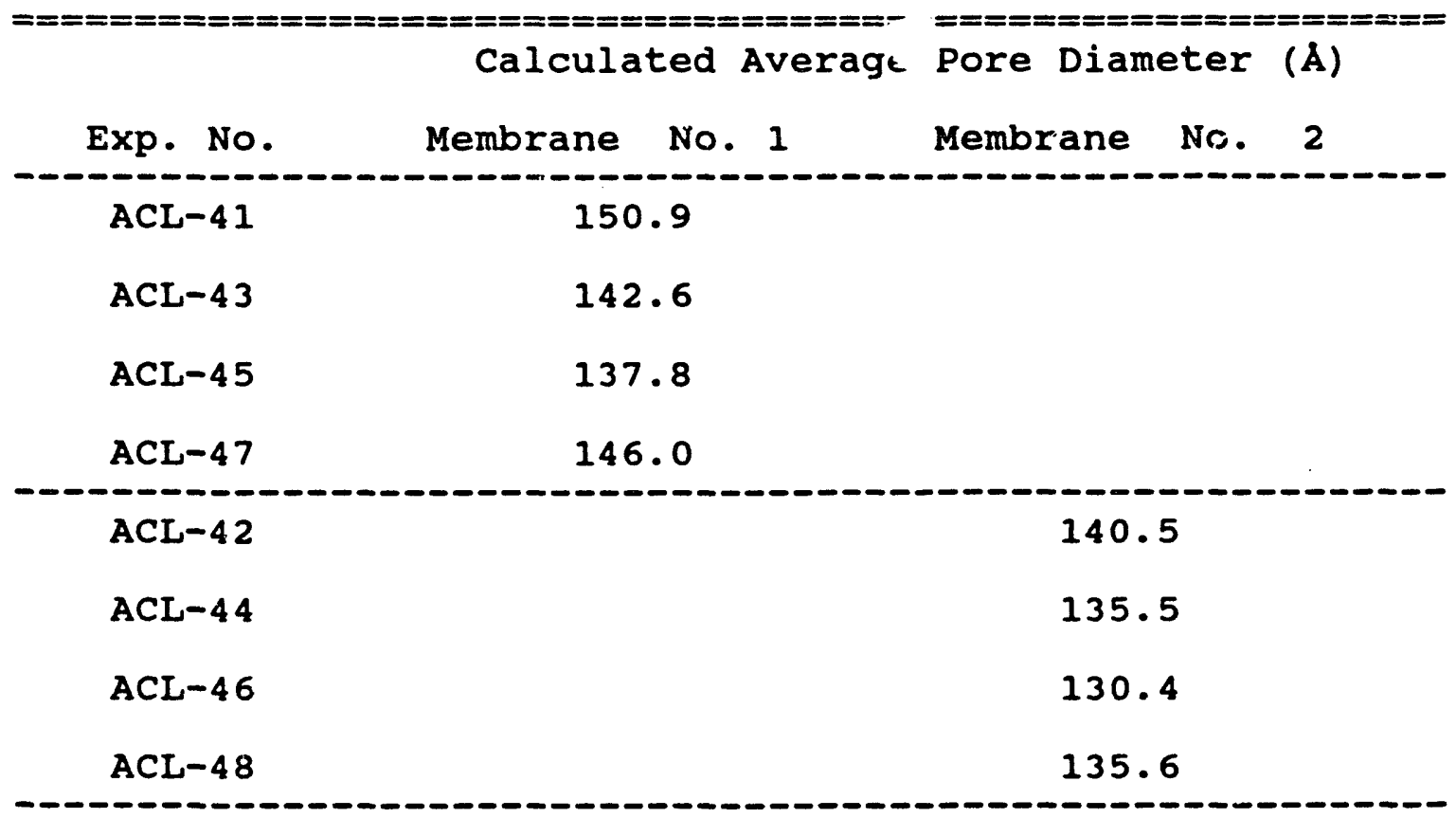


table, the pore diameters decreased as coal diffusion experiments were repeated and increased again in the last octane diffusion experiment. Detailed discussion concerning this observation will be made in the next section. At the moment, an important point of this observation is that pore diameters of membranes kept changing during the coal diffusion experiments. Thus, by using the in situmeasurement technique, the accuracy of diffusion experiments could be improved.

A question might be posed concerning the effect of $n$ octane introduced as a tracer on the diffusivity measurements of coal macromolecules. However, the binary system analogy of multicomponent diffusion was already proved experimentally in the previous model compounds study. Since coal was already a complex mixture of various compounds, and their concentration levels in the diffusion cell were low enough that the solutes would behave ideally (no solute-solute interactions to enhance or retard diffusional flux by coupling, etc.), the introduction of one more hydrocarbon, with no functional group, to the coal asphaltene diffusion system should not alter the resilts of the diffusion experiments.

The bulk diffusivities (or molecular diffusivities) of coal macromolecules were measured with commercial polyester membranes having a $0.1 \mu \mathrm{m}$ nominal pore size (Nuclepore Co.). The average pore size of these membranes, determined from 
the diffusivity measurement of $n$-octane, was found to be $0.126 \mu \mathrm{m}$. Since coal asphaltene consists of various size molecules, hinderance factors for some coal molecules were expected to be significant with these relatively large pore size membranes. Thus, the hinderance factor was taken into account in the analysis of experimental data. Experiments were conducted using the indirect measurement method described in Chapter VI, i.e., the bulk diffusivities of coal macromolecules' fractions were determined using the diffusivity of n-octane in ethyl acetate as a reference value.

In the concentration analysis work using GPC, UV absorbance data were automatically transmitted to a computer through a data acquisition board (Metrabyte Co., Model DAS8PGA) and processed by the computer (IBM PC Model 30) to improve the accuracy of the analysis. Computer programs used for this data collecting and processing are listed in Appendix G.

In the analysis of experimental results, six fractions of coal macromolecules were defined first in the chromatogram of the original stock solution according to their GPC elution volumes. The width of the whole range for the six fractions was selected to cover the significant molecular weight range of coal asphaltene (from $25 \mathrm{ml}$ to 37 $\mathrm{ml}$ in Figure 7.2), and this range was evenly cut into six fractions. Then the bulk diffusivity and effective 
diffusivity of each fraction were analyzed separately from the GPC elution curves of the samples collected from the top and bottom compartments of the diffusion cell.

\section{- Results and Discussion}

One important assumption used in the analysis of the current experimental data was that the area of each fraction under the curve in the chromatogram corresponded to the concentration of each coal fraction in the solution.

Two questions arose concerning this assumption; one was whether the large area in the chromatogram was necessarily an evidence of a large mass existence, because it was likely that each coal molecule had a different extinction coefficient; the other was whether there were structural rearrangements of coal molecules during the diffusion experiment or the GPC analysis, since it is well known that coal asphaltene in solution are usually associated (126). In an effort to answer these questions, the standard sample solutions of the coal macromolecules having different concentrations were prepared, and GPC analyses were conducted. From the results of the GPC analyses of these standard solutions, the area of each fraction in the chromatogram was plotted against the concentration of each solution. The results are presented in Figure 7.3. As shown in this figure, good linear relationships between the areas of coal fractions and the concentrations of sample 


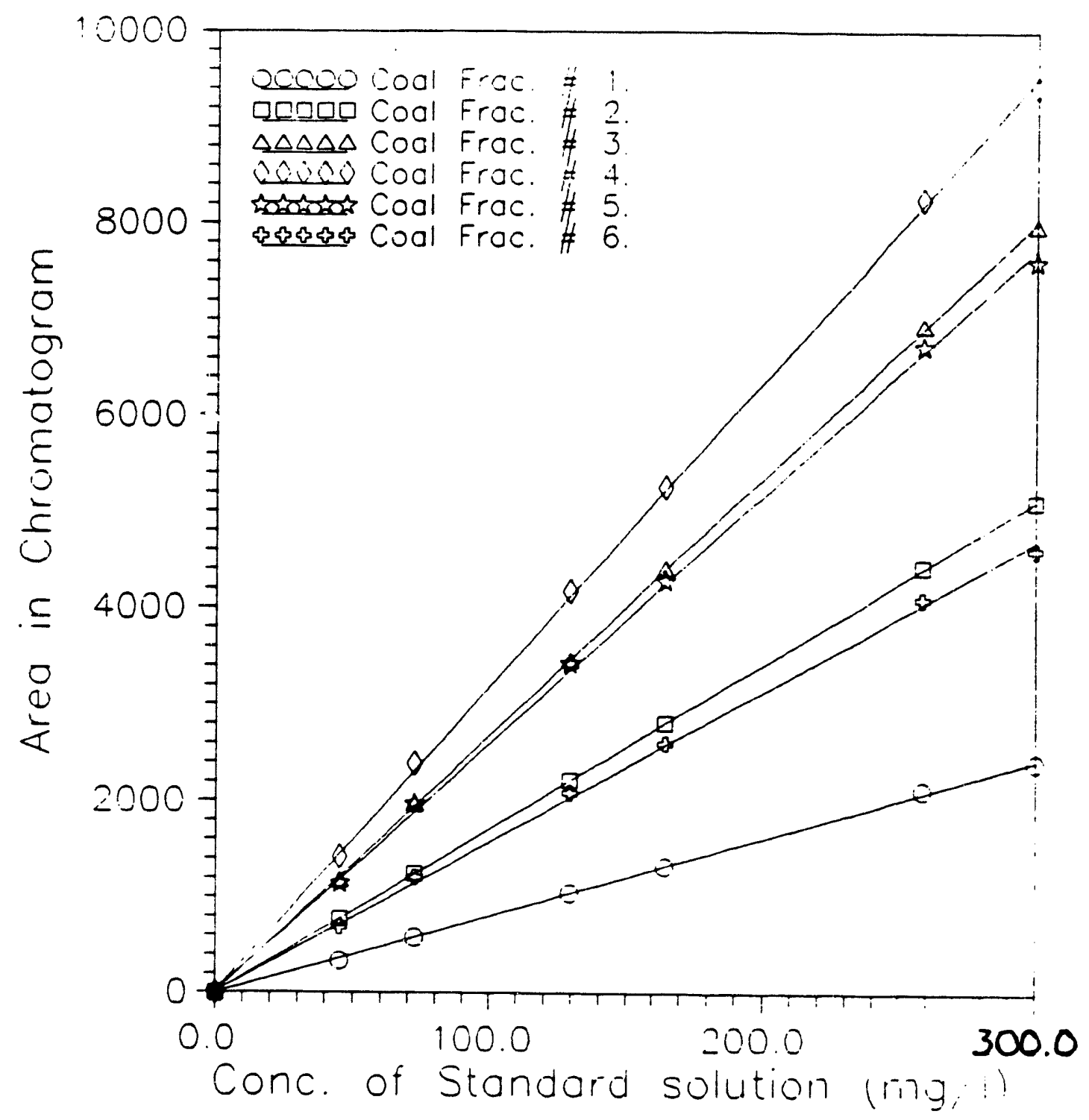

Figure 7.3 Area of Each Coal Fraction in Chromatogram vs. Concentration of standard solution 


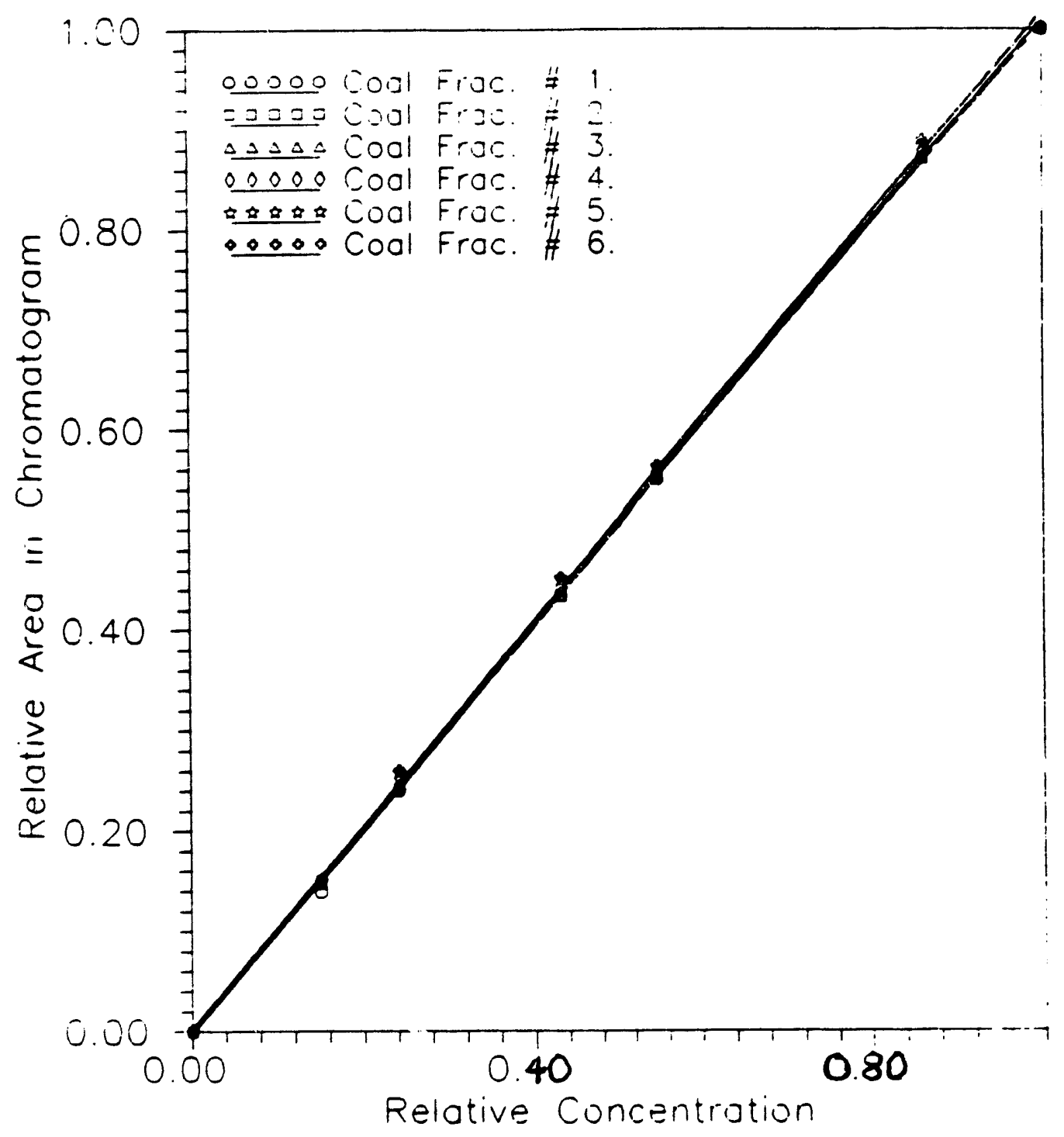

Figure 7.4 Relative Area vs. Relative Concentration 
solutions were observed. The implication of these results can be more easily understood in Figure 7.4, where the ratios of the concentrations of standard solutions to that of the stock solution are plotted against the ratios of chromatogram areas of coal fractions obtained from the standard solutions to those from the stock solution. As shown in this figure, the values of relative concentrations are the same as those of the relative areas for all coal fractions. The results indicated that the change in each coal fraction's area of chromatogram could sufficiently represent the change in concentration of each fraction during the diffusion experiments, even though the exact mass concentration of each fraction could not be measured. Thus, the assumption used in the current analysis was acceptable. As noted previously, the bulk phase diffusivities for coal macromolecules' fractions were measured using the indirect measurement method. In the analyses of experimental data, Equation 6.2 was used along with Equation 2.20 for the hinderance factor of each coal fraction and Equation 5.5 for the boundary layer resistance. The results of are summarized in Table 7.4. As shown in the table, the bulk diffusivities decreased as the equivalent molecular weights of coal molecules increased. The standard deviations of bulk diffusivities were somewhat largr compared to those of model compounds' diffusivities. These relatively large experimental errors appeared to be due to 
Table 7.4

Bulk Diffusion Coefficients of coal Asphaltene Fractions

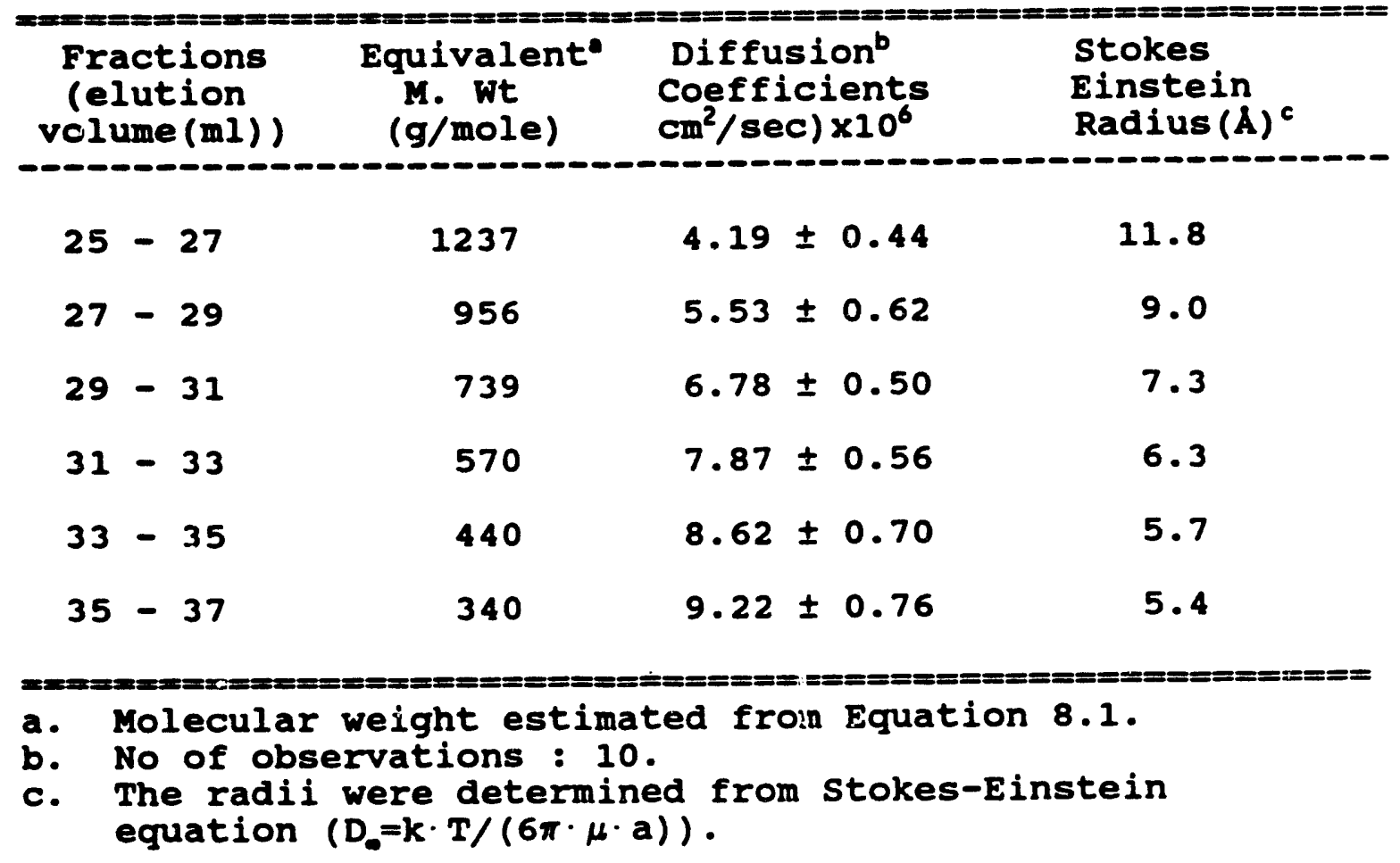


the errors in GPC analysis and the errors associated with the charging of the stock solution.

In comparing the diffusivities measured in the current experiments with those of model compounds, the diffusivity value of TPP (MW: $614.8, D_{\odot}: 6.83 \times 10^{-6} \mathrm{~cm}^{2} / \mathrm{sec}$ ) reasonably fell between that of the coal fraction having equivalent molecular weight 739 and that of the fraction having equivalent molecular weight 570. However, the diffusivities of rubrene and coronene obtained in the model diffusion study somewhat higher than those of equivalent molecular weight coal molecules. A direct explanation for this observation could not be given. However, it is likely that different partitioning behaviors of coal molecules from those of rubrene or coronene in the GPC column may be one of the reasons for the differences in the bulk diffusivities, since coal molecules have many functionalities $(69,122)$.

The results of the effective diffusivity measurements of coal molecules are summarized in Table 7.5. To obtain the effective diffusivities of coal fractions from the experimental data, Equation 2.2 was used. As previously noted, average pore sizes of membranes required for the calculations using Equation 2.2 were determined from in situ-diffusivity measurement of n-octane. In Table 7.5, average pore diameters of the membranes used in the experiments are also presented. 
Table 7.5

\section{Effective Diffusion Coefficients of Coal Asphaltene Fractions.}

\begin{tabular}{|c|c|c|c|c|}
\hline $\begin{array}{l}\text { Fractions } \\
\text { (Elution } \\
\text { volume } \\
\text { (ml)) }\end{array}$ & $\begin{array}{l}\text { Effecti } \\
\text { run-01a } \\
(119 A)^{b}\end{array}$ & $\begin{array}{l}\text { iffusion } \\
\text { run-02 } \\
(103 \text { A) }\end{array}$ & $\begin{array}{l}\text { fficient } \\
\text { run-15 } \\
(110 \mathrm{~A})\end{array}$ & $\begin{array}{l}\left.\mathrm{cm}^{2} / \mathrm{sec} \times 10^{6}\right) \\
\text { run-16 } \\
(123 \AA)\end{array}$ \\
\hline $25-27$ & 0.93 & 1.12 & 0.93 & 0.99 \\
\hline $27-29$ & 1.65 & 1.30 & 1.47 & 1.34 \\
\hline $29-31$ & 2.30 & 1.91 & 2.42 & 1.99 \\
\hline $31-33$ & 3.54 & 2.92 & 3.11 & 2.73 \\
\hline $33-35$ & 4.46 & 3.89 & 4.02 & 3.63 \\
\hline $35-37$ & 5.04 & 4.87 & 5.00 & 4.71 \\
\hline
\end{tabular}

\begin{tabular}{|c|c|c|c|c|}
\hline \multirow{2}{*}{$\begin{array}{l}\text { Fractions } \\
\text { (Elution } \\
\text { volume } \\
(m l))\end{array}$} & \multicolumn{4}{|c|}{ Effective diffusior } \\
\hline & $\begin{array}{r}\text { run-17 } \\
(199 \AA)\end{array}$ & $\begin{array}{l}\text { run-22 } \\
(259 \AA)\end{array}$ & $\begin{array}{l}\text { run-28 } \\
(135 \AA)\end{array}$ & $\begin{array}{l}\text { run-36 } \\
(436 \AA)\end{array}$ \\
\hline $25-27$ & 1.35 & 2.43 & 0.86 & 3.04 \\
\hline $27-29$ & 2.27 & 3.43 & 1.67 & 4.25 \\
\hline $29-31$ & 3.08 & 4.47 & 2.62 & 5.39 \\
\hline $31-33$ & 3.91 & 5.66 & 3.76 & 6.58 \\
\hline $33-35$ & 4.77 & 6.72 & 4.72 & 7.59 \\
\hline $35-37$ & 5.49 & 7.49 & 6.09 & 8.27 \\
\hline
\end{tabular}


Table 7.5. Continue...

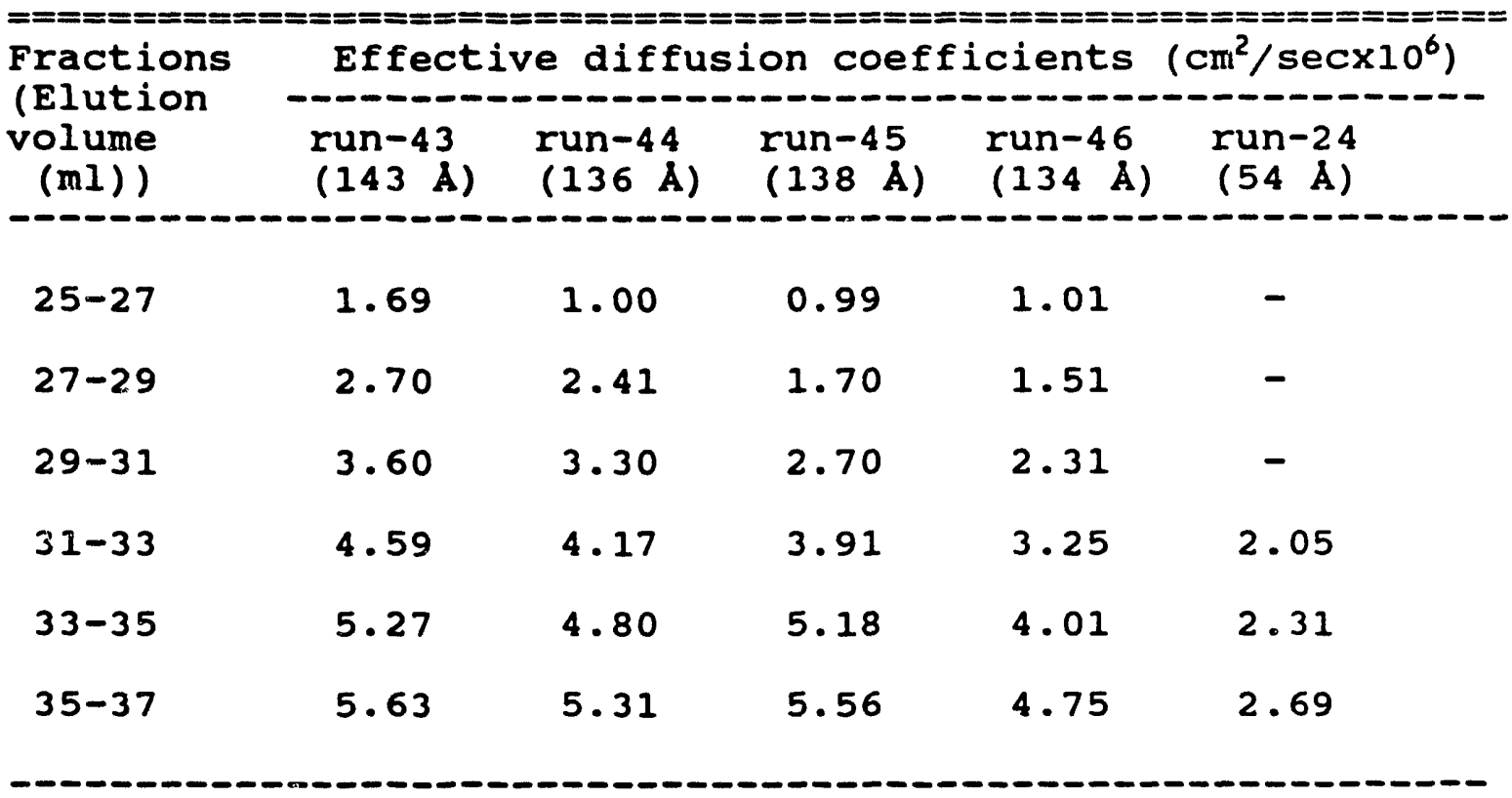

a. Experiment number.

b. Average pore diameter determined from the results of n-octane diffusivity measurement. 
With the results shown in Tables 7.4 and 7.5 , the ratios of effective diffusivities to bulk diffusivities are plotted against the ratios of molecular sizes to the average pore sizes in Figure 7.5. Here, the molecular size of each fraction was calculated from the bulk diffusivity by using stokes-Einstein equation $\left(r_{s}=k \cdot T /\left(6 \pi \cdot \mu \cdot D_{0}\right)\right.$. The same theoretical equation used in the analysis of experimental data of TPP was adopted to evaluate the experimental data. The solid line drawn in Figure 7.5 represents the theoretical data calculated from Equation 6.3. As shown in this figure, the experimental data fall below the theoretical curve. The results were somewhat contrary to that of TPP, where experimental data fell above the theoretical curve. In order to evaluate the significance of the deviation between the experimental data and the theoretical curve, the same statistical methods used to evaluate the experimental results of TPP, as described in Chapter VI, were carried out.

The results are summarized in Table 7.6. The model parameters for the theoretical curve were the same as those obtained previously. As shown in this table, all statistical studies indicated that signiticant differences existed between the theoretical curve and the experimental data. In order to explain these differences, it should be noted here that the pore diameters of membranes decreased during the hindered diffusion experiments of coal 


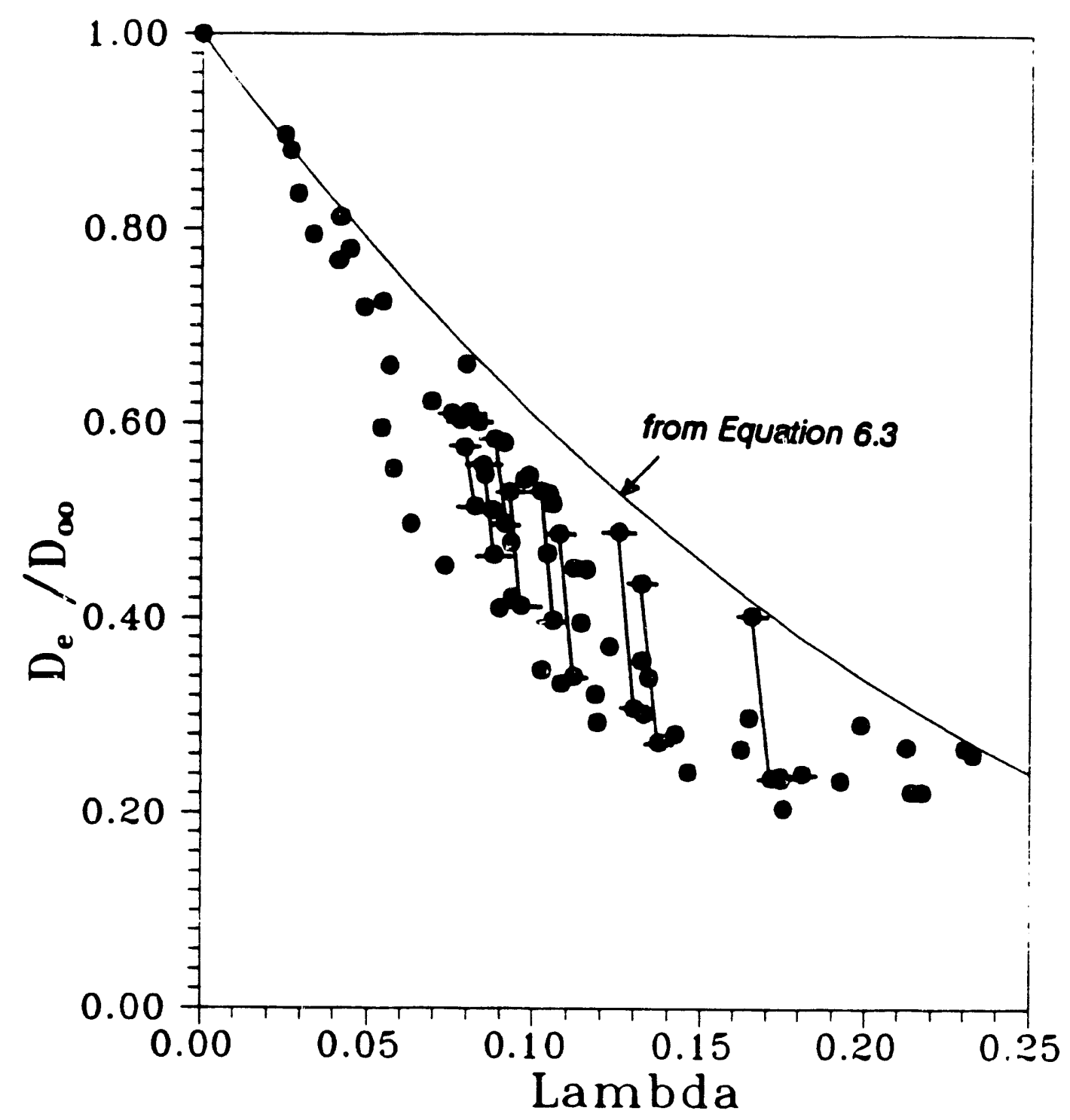

Figure 7.5 Dependence of $D_{e} / D_{0}$ on $\lambda$ in Hindered Diffusion of Coal Asphaltene 


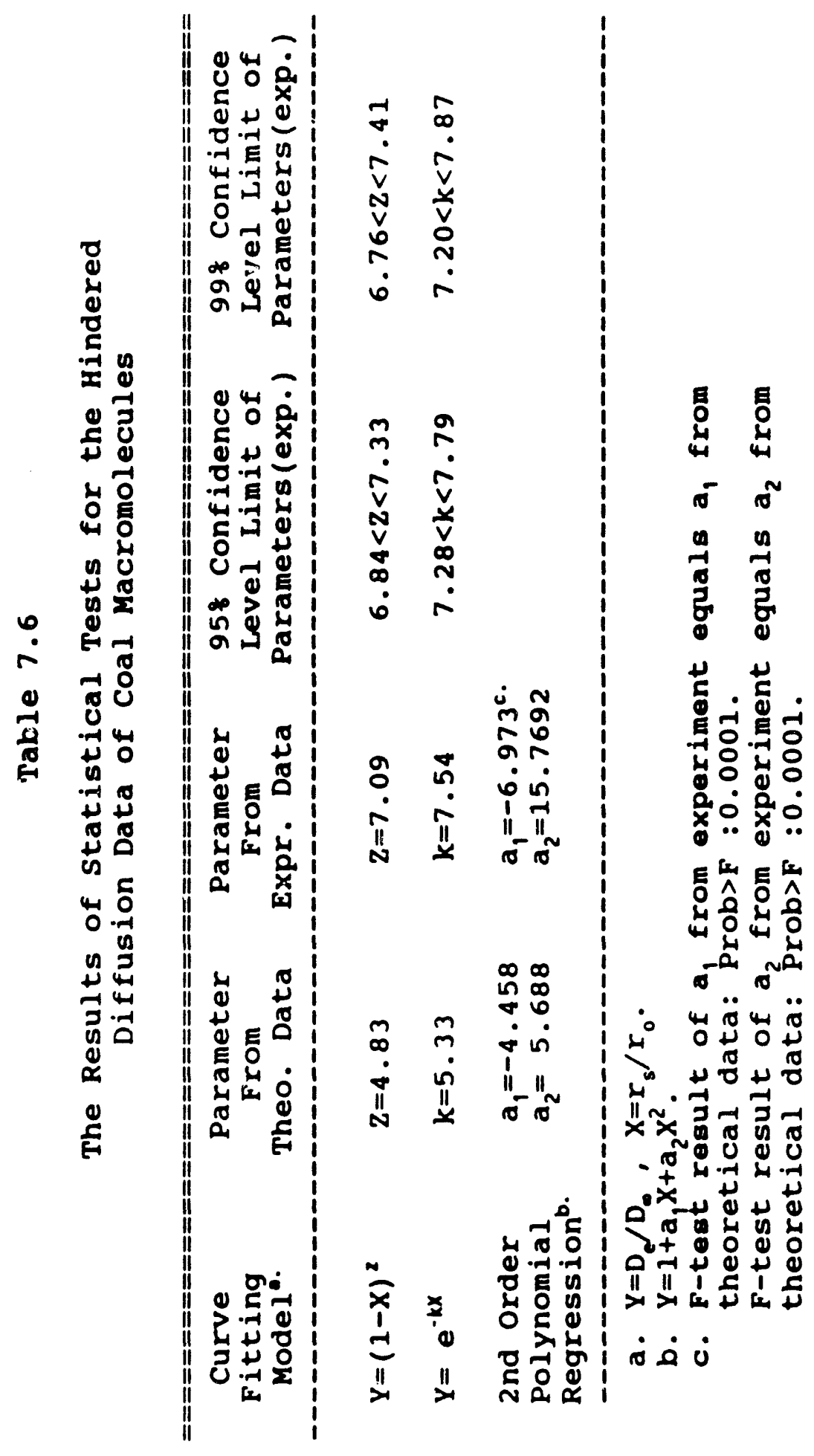


macromolecules. It should also be noted here that the true pore radii values obtained from the in situ diffusivity measurement of n-octane were used in Figure 7.5. As described previously, it was surmised that the change in pore diameters was due to the adsorption of coal molecules to the pore walls. This hypothesis becomes apparent when the chemistry of coal asphaltene is examined more closely. As reviewed in chapter II, coal asphaltene model proposed by Yen (122) interestingly postulated the acidic and basic nature of coal asphaltene. The acid-base structure of coal asphaltene was also demonstrated by sternberg et al. (123). They separated the coal asphaltene into acid-neutral fractions and base fractions by dissolving the asphaltene in toluene and passing dry $\mathrm{HCl}$ gas through the solution. The basic component was precipitated as an insoluble adduct while the acidic and the neutral components remained in the solution. The authors reported that the expected functional groups in the acidic components were phenolic $\mathrm{O}-\mathrm{H}$ and pyrrolic $\mathrm{N}-\mathrm{H}$, while those in the basic components were ring oxygen as in furan or ring nitrogen as in pyridine; these components were responsible for the hydrogen bonding. They thought that the acidic and the basic components were separately solvated in solvents such as benzene. The acidbase structure of coal asphaltene was also proved by sidney et al. using infrared spectrometry (124). 
Although the model of coal asphaltene structure studied in these works did not quite agree with the model proposed by Yen, both models acknowledged the existence of hydrogen bonding between acidic and basic functional groups in coal asphaltene.

All of the aforementioned studies implied that either acidic or basic components (or functionalities) of coal asphaltene solvated in ethyl acetate might selectively adsorb to the pore walls of membranes in the current hindered diffusion experiments. The amount of adsorbed coal molecules was supposed to be very small (as seen in Table 7.3), far below the amount required to produce a monolayer, since the concentration of coal solution used in the experiments was extremely low (ca. $300 \mathrm{mg} / 1$ ). Yet, these adsorbed molecules were likely to interact with other coal molecules transferring through the pore; these interactions were expected to be an electrostatic repulsive interaction. The degree of interactions would not be strong because of the low concentration used in the current study, as well as the characteristics of coal asphaltene (e.g., the existance of neutral components according to the sternburg et al.'s study). Nevertheless, this repulsive interaction could decrease the partition coefficient, thus making the experimental data fall below the theoretical curve derived with the no pore-molecule interaction assumption. 
The effect of electrostatic repulsive interactions on the partition coefficient has been investigated in several previous studies $(31,106,107,125)$. Among these studies, the experimental study conducted by Rodilosso drew attention because of the phenomenal similarity found in his experimental results and the current study. He measured the partition coefficient for bovine serum albumin (BSA, $r_{s} \approx$ $36 \mathrm{~A}$ ) in a track-etched mica membrane which had pore walls precoated with BSA by adsorption (experimental conditions: dilute solution of $\mathrm{BSA}$ at $\mathrm{pH} 8.5$ and $0.2 \mathrm{M}$ ionic strength). He reported that the partition coefficient was found to be at least one order of magnitude lower than the theoretical curve obtained from simple steric exclusion; he explained that these observations were due to the electrostatic repulsive interactions between $B S A$ molecules in the solution and the BSA-coated pore wall. The nature of the elestrostatic interactions found in his study might be different f:om those observed in the current research since most protein molecules were charged depending on the $\mathrm{pH}$ of the solution. However, it was apparent that the interactions bitween adsorbed molecules and transferring molecules througcin the pore decreased the partition coefficient, and that these interactions were due to the existence of functional groups in the molecules. All of these findings support the hypothesis used to explain the phenomenon observed in Figure 7.5 . 
Before any final conclusions concerning the observations seen in the hindered diffusions of coal macromolecules are made, relatively large experimental errors found in the bulk diffusivity measurements should be discussed. As seen in Table 7.3, the standard deviations of bulk diffusivity value reached about 10\%. Experimental errors also existed in the effective diffusivity measurements. In Figure 7.5 , the lines connecting two points represent the experimental data obtained from two consecutive hindered diffusion experiments conducted with the same polyester membrane. As seen in this figure, experimental data points were not completely reproducible. Again, it was suspected that these experimental errors were due to the errors in GPC analysis. Errors caused by using the nominal value of pore densities in the analysis of experimental data were turned out to be negligible as reported in Appendix $\mathbf{F}$.

These experimental errors could be large enough to mask the differences between the experimental data and the theoretical curve observed in Figure 7.5. However, it is the scatter of experimental data that is affected by experimental errors. As seen in Figure 7.5, the current experimental data do not scatter randomly around the theoretical curve. They scatter below the theoretical curve and the significance of deviation from theoretical curve was already examined by the statistical methods. Thus, it is 
likely that the previously drawn conclusion, relating the strong hindrance of coal molecules to electrostatic repulsion, is the reason why the coal macromolecules are more hindered than the theoretical curve predicted by the neutral hard sphere theory.

\section{Diffusion in Actual Alumina Porous Media}

We have extended our configurational diffusion results from idealized membranes with uniform pores to actual tortuous porous media. To do this, we used a finite bath diffusion apparatus and methodology developed in our laboratory (11). The use of this previously developed technique resulted in time savings and promoted greater efficiency in the collection of project data. Experiments were performed with both TPP and coal macromolecules at ambient and elevated temperatures. The effect of elevated temperature on the diffusion of TPP and coal macromolecules was small. A detailed discussion of the results of these experiments is presented in Appendix $\mathrm{H}$. 


\section{SUMMARY AND CONCLUSIONS}

To provide basic information for understanding the complex nature of diffusional phenomena occurring in catalytic coal liquefaction, a fundamental diffusion study of coal macromolecules using track-etched membranes was conducted in the current research.

Initial research activities were focused on the preparation and characterization of the track-etched membranes. After the successful completion of this study, the research was centered on a diffusion study of model compounds to test the performance of experime:tal system and to provide fundamental information as to the effect of molecular size and configuration on the effective diffusivity. Prior to this study, a diffusional boundary layer study was also conducted, since the effective diffusivity of a solute was evaluated from the intrinsic membrane resistance wile experimentally the total transfer resistance, which was the summation of the intrinsic membrane resistance and the diffusional boundary layer resistance, was the only measurable quantity from a diffusion experiment. Finally, a hindered diffusion study of coal macromolecules was performed. 
The major conclusions of the current research are as

\section{follows:}

- The bulk diffusivities of coal fractions decreased as the equivalent molecular weight of each fraction increased. As shown in Table 7.4 , their values ranged from $4.19 \mathrm{X}$ $10^{-6} \mathrm{~cm}^{2} / \mathrm{sec}$ to $9.22 \times 10^{-6} \mathrm{~cm}^{2} / \mathrm{sec}$. The variation in bulk diffusivities of coal asphaltene fractions was smaller than that of petroleum asphaltene fractions (one order of magnitude variation (51)).

- The measured effective diffusivities of coal fractions generally followed the trend predicted from the hydrodynamic theory of hindered diffusion. However, the results were opposite to that of model macromolecule (TPP), where experimental data fell above the theoretical curve (see Figures 6.5 and 7.5 ).

- The differencies between the experimental effective diffusivity data of coal macromolecules and the theoretical curve (see Figure 7.5) were statistically significant. Weak repulsive electrostatic interactions between adsorbed coal molecules and transferring coal molecules through the pore were postulated to account for these differencies, since some coal molecules appeared to adsorb to the pore wall of polyester membrane during the diffusion experiments due to their acidic or basic functionalities. According to Equation 6.5, an introduction of repulsive interaction make the partition 
coefficient $\left(K_{p}\right)$ decrease and experimental data points for $D_{e} / D_{0}$ move downward from the theoretical curve.

Additional conclusions drawn from the results of the current research are summarized below.

- The etching rate of irradiated polycarbonate films were dependant on the concentration and the temperature of etching solution.

- with a constant temperature and concentration of etching solution, the etching process of irradiated polycarbonate film proceeded quite linearly with the etching time.

- The activation energy of the etching process used in the current study was $0.78 \mathrm{ev}$.

- When a membrane having small-sized pores was prepared, controlling the etching temperature was more favorable than controlling the concentration of etching solution from an experimental standpoint.

- Track-etched polycarbonate membranes were not suitable for the hindered diffusion study of coal macromolecules due to their chemical incompatibility with ethyl acetate used as a solvent in the coal diffusion experiments.

- Track-etched polyester membranes having small size pores, which could cause appreciable hindrance effects for the coal macromolecules diffusing through pores, were successfully prepared with following etching conditions: $1 \mathrm{~N}$ NaOH solution with 0,02 wt\% Dowfax ${ }^{\mathrm{TM}} 2 \mathrm{Al}$ 
surfactant, $80^{\circ} \mathrm{C}$ etching temperature, relatively short etching times (less than 5 min.)

- In spite of the possible advantages of alumina membranes over track-etched polymer membranes associated with the current diffusion study, attempts to prepare anodic oxidized alumina membranes suitable for hindered diffusion experiments of coal macromolecules were not successful, since the membrane became too thin and fragile at process conditions favorable for the formation of small size pores.

- The scanning electron microscope pictures of track-etched polymer membranes revealed that the pore openings in these membranes were circular, the pores were identical and the pore densities did not deviate more than $\pm 10 \%$ from their nominal values.

- Pore sizes of a track-etched membrane separately determined from the liquid permeability test, the gas permeability test and the diffusivity measurement of a solute of known diffusivity were in close agreement with one another.

- An empirical equation describing the diffusional boundary layer behavior in the diffusion cell used in the current study was found and expressed as:

$$
\mathrm{N}_{\text {Sh }}=0.084 \mathrm{~N}_{\mathrm{Re}}^{0.68} \mathrm{~N}_{\mathrm{SC}}^{0.33}
$$

- The boundary layer resistance was not affected by the the membrane properties, in praticular the porosity. 
- The pores in alumina membranes appeared to have tortuosities greater than unity based on the diffusion experimental results using commercial alumina membranes.

- The diffusivities of three paraffinic hydrocarbons (n-octane, n-dodecane, n-tetradecane) in n-decane measured with the current experimental system agreed with the values found in the literature.

- The diffusivities of model solutes measured with an indirect measurement methods were in close agreement with those measured with the direct measurement method; the results supported the binary component diffusion analogy for the multicomponent diffusion at dilute concentration range.

- The diffusivity of n-octane in ethyl acetate measured in the current study was $2.47 \times 10^{-5} \mathrm{~cm}^{2} / \mathrm{sec}$. This measured diffusivity value was reasonably close to the predicted values calculated from the several estimation methods in the literature.

- The measured diffusivities of polycyclic aromatic hydrocarbons in ethyl acetate agreed with the estimated values from the several methods in the literature and correlated well with the average planar molecular radii, which were defined as the geometric average of longest planar dimensions and the shortest planar dimensions of molecules. 
- The measured bulk diffusivity of TPP in ethyl acetate agreed with the estimated value calculated from Anderson et al.'s experimental diffusivity data of TPP in chloroform (89) using the Wilke-Chang equation.

- The measured effective diffusivity of TPP generally followed the trend predicted from the hydrodynamic theory of hindered diffusion.

- The differences between the experimental data and the theoreticai curve seen in Figure 6.5 for the effective diffusivity of TPP were statistically significant. A weak attractive interaction between TPP molecule transferring through the pore and the pore wall was considered to be responsible for these differences.

- A correlation equation, to estimate the equivalent molecular weights of coal fractions, obtained from the GPC calibration study using TPP, polystyrene standards and polycyclic aromatic hydrocarbons was expressed as:

$$
\log _{10} M_{w}=-0.56 v_{i}+4.55
$$

- The effect of elevated temperature on the diffusion adsorption uptake of TPP and coal macromolecules in alumina pellets did not appear to be pronounced. 
IX. RECOMMENDED AREAS OF FUTURE WORK

One of the major objectives of the diffusion study in the area of coal liquefaction catalysis is to obtain an optimum pore size or optimum pore size distribution of catalyst which accounts for the trade-off situation between surface area and diffusional accessibility.

In the current research, the bulk diffusivities of coal macromolecules at room temperature conditions were measured, and the relationship between effective diffusivities of coal macromolecules and pore size were evaluated using tracketched polyester membranes which simulated idealized pores in catalysts.

Although the results from the current research improve the understanding concerning the diffusional phenomena occurring in coal liquefaction catalysts, the results are not directly applicable to describe diffusion in coal liquefaction catalysis. Based on the work performed in the current study, the following research areas are recommended for future studies.

- Measurements of the effective diffusivities of coal macromolecules using extrudate-type catalysts. 
- Counter-hindered-diffusion study with model macromolecules.

- Study on the temperature and solvent dependencies of the bulk diffusivities and effective diffusivities of coal macromolecules.

- Hindered diffusion study of a model macromolecule using anodic oxide alumina membranes.

- Measurement of effective diffusivities of coal asphaltene from different sources.

- Measurement of effective diffusivities of acidic components and basic components of coal asphaltene. 


\section{BIBLIOGRAPHY}

1. Rhee, Y., Ph.D. Thesis, Auburn University, Auburn, 1989

2. Ho, P. N. and Weller, S. W., Fuel Processing Technol., 4, 21, 1981

3. Kang, C. C., Proceedings, 1983 International Conference on Coal Science, Pittsburg, 41, 1983

4. Ternan, M., Can. J. Chem. Eng., 61, 689, 1983

5. Yen, Y. K., Furlani, D. E., and Weller, S. W., Ind. Eng. Chem. Prod., Res.\&Div., 15(1), 24, 1976

6. Shimada, H., Kurita, M., Sato, T., Yoshimura, Y., Kawakami, T., Yoshitomi, S. and Nishijina, A., Bull. Chem. Soc. Jpn., $57,2000,1984$

7. Curtis, C. W., Guin, J. A., Kamajian, B. L. and Moody, T. E., Fuel Processing Technol., 12, 111, 1986

8. Curtis, C. W., Tsai, K. J. and Guin, J. A., Ind. Eng. Chem. Res, 26, 12, 1987

9. Stiegel, G. J., Polinski, L. M. and Tischer", R. E., Ind. Eng. Chem. Proc. Des. Dev., 21, 277, 1982

10. Curtis, C. W., Guin, J. A., Tarrer, A. R. and Hwang, W., Fuel Processing Technol., 7, 277, 1983

11. Tsai, K. J., Ph.D. Thesis, Auburn University, Auburn, 1985

12. Gollakota, S. V., Guin, J. A. and Curtis, C. W., Ind. Eng. Chem. Proc. Des. Dev., 24, 1148, 1985

13. Steedman, W., Fuel Processing Technol., 10, 209, 1985

14. Komiyama, H. and Smith, J. M., AIChE J., (20)4, 728, 1974

15. Froment, G. F. and Bischoff, K. B., Chemical Reactor Analysis and Design, John Wiley \& Sons, New York, 1979

16. Tsotsis, T. T., Sane, R. C., Webster, I. A. and J. D. Goddard, J. of catalyst, (101), 416, 1986 
17. Northrop, J. H. and Anson, M. L., J. Gen. Physical, $12,543,1929$

18. McBain, J. W. and Liu, T. H., J.Amer. Chem. Soc,, 53, 59,1931

19. Gordon, A. R. Annals of N. Y.Acad. Sci, 46, 285, 1945

20. Hartley, G. S. and Runnicles, D. F., Proc. Royal Soc., A168, 40, 1938

21. Stokes, R. H., J. Amer. Chem. Soc., 72, 2243, 1950

22. Cussler, E. L. Diffusion. Mass transfer in fluid systems, Cambridge University Pres. Cambridge, 1984

23. Robinson, R. L., Edmister, W. C., Dullien, A. L., J. Phys. Chem., 69, 258, 1965

24. Janz, G. J. and Mayer, G. E., Research and Development Progress Report N. 196, U. S. Department of Interior, 1966

25. Malone, D. M. and Anderson, J. L., Chemical Engineering Science, (33), 1429, 1978

26. Deen, W. M., Bohrer, M. P. and Epstein, N. B., AIChE J., (27) $6,952,1981$

27. Bohrer, M. P., Ind. Eng. Chem. Fundam., (22), 72, 1983

28. Bohrer, M. P., Patterson, G. D. and Carroll, P. J., Macromolecules, (17), 1170, 1984

29. Baltus, R. E. and Anderson, J. I., Chemical Engineering Science, (38) 12, 1959, 1983

30. Guillot, G., Macromolecules, (20), 2600, 1987

31. Malone, D. M. and Anderson, J. L., AIChE J., (23) 2,177 , 1977

32. Kaufmann, T. G. and Leonard, E. F., AIChE J., (14) 3, 421, 1968

33. Smith, K. A. and Colton, C. K., AIChE J., (18) 5, 949, 1972

34. Levich, V. G., Physiochemical Hydrodymics, Prentice Hall, Inc., Englewood Cliffs, NJ. 1962

35. Ferry, J. D., J. Gen. Physiol, 20, 95, 1936 
36. Faxen, H., Arkiv, Mat. Astron. Fys,, 17(27), 1923

37. Pappenheimer, J. R., Renkin, E. M. and Borrero, L. M., Amer. J. Physiol, 167, 13, 1951

38. Renkin, E. M., J. Gen. Physiol, 38, 225, 1954

39. Deen, W. M., AIChE J., (33) 9, 1409, 1987

40. Brenner, H. and Gaydos, L. T., J. Colloid. Interf. Sci., $58,312,1977$

41. Giddings, J. C., Kucera, E., Russell, C. P. and Myers, M. N., J. Phys. Chem., 72, 4392, 1988

42. Kathawalla, I. A., Anderson, J. L. and Lindsey, J. S., Macromolecules, (22), 1215, 1989

43. Mavrovouniotis, G. M. and Brenner, H., AIChE Ann. Meeting, Paper No 85b, Miami, 1986

44. Colton C. K., Satterfield, C. N. and Lai, C., AIChE J., (21) $2,289,1975$

45. Bishop, M. T., Langley, K. H. and Karasz, F. E., Macromolecule, (22), 1220, 1989

46. Conlon T. and Craven, B., Aust. J. Chem., (25), 695, 1972

47. Cannell, D. S. and Rondelez, F., Macromolecules, (13), 1599,1980

48. Chantong A. and Massoth, F. E., AIChE J., (29) 5, 725 , 1983

49. Shimura, M., Shiroto, Y., Takeuchi, C., Ind. Eng. Chem. Fundam., (25), 330, 1986

50. Ackers, G. K. and Steere, R. L., Biochim. Biophys. Acta., (59), 137, 1962

51. Baltus, R. F. and Anderson, J. L., Chemical Engineering Science, (38) 12, 1959, 1983

52. Sane, R. C., Tsotsis, T. T. and Vebster I. A., Amer. Chem. Soc. Prep. Fuel Chem. Div., 33(3), 237, 1988

53. Baltus, R. E., Kyriacou, K. C., Sivaramakrishna, V. V., and Rahimi, P., AIChE Symposium Series, (84)266, 50, 1986

54. Beck, R. E. and Schultz, J. S., Biochim. Biophys. Acta, $(255), 273,1972$ 
55. Moore, R. M., Katzer, J. R., AIChE. J., $18(4), 816,1972$

56. Satterfield C. N., Colton, C. K. and Pitcher, Jr., W. H., AIChE J., (19) 3, 628, 1973

57. Wong, J. H. and Quinn, J. A. Collid \& Interface Science, (5), M. Kerker, Academic Press, 1975

58. Prasher, B. D. and Ma Y. H., AIChE J., (23) 3, 303, 1977

59. Prasher, B. D., Gabriel, G. A. and Ma, Y. H., AIChE J., (24) $6,1118,1978$

60. Glandt, E. D., AIChE J,, (27) 1, 51, 1981

61. Price, P. B. and Walker, R. M., J. of Applied Physics, (33) $12,3400,1962$

62. Price, P. B. and Walker, R. M., J. of Applied Physics, (33) $12,3407,1962$

63. Bean, C. P., Doyle, M. V. and Entine, G., J. of Applied Physics, (41) 4, 1454, 1970

64. Komaki, Y. and Seguchi T., Polymer, (23), 1143, 1982

65. Desorbo, W., Nucl. Truacks, (3), 13, 1979

66. Bean, C. P., Doyle, M. V., and Entine, G., J. of Applied Physics, (41) 4, 1970, 1454-1459

67. Guillot, G. and Rondelez, F., J. Appl. Phys., 52 (12), 7155,1981

68. Quinn, J. A., Anderson, J. L., Ho, W. S. and Petzny, W. J., Biophysical Journal, (12), 990, 1972

69. Bunger, J. W., Li, C. N., Chemistry of Asphaltene, Advances in Chemistry Series \#195, American Chemical society, washington D. C., 1981

70. Gorbaty, M. L. and ouchi, K., coal structure, Advances in Chemistry Serise \#192, American Chemical Society, Washington D. C., 1981

71. Itaya, K., Sugawara, S., Arai, K. and Saito, S., J.Chem. Eng. Japan, $17(5), 514,1984$

72. Schultz, J. S., Valentine, R. and Choi, C. Y., J. Fen. Physiol, (73), 49, 1979 
73. Anderson, J. L., Ph.D. Thesis, Univ. of Illinoids, Urbana-Champaign, 1971

74. Yoon, J. H. and Kang, T., Kumsok Pyomyum Choli, 13 (4), 221,1980

75. Keller, F., Huntr, M. S., Robinson, D. L., J. Electrochem. Soc., $100(9), 411,1953$

76. Chiou. Z., Ke S. Thesis, Auburn University, Auburn, 1989

77. Williamson, B. G. and Geankoplis, C. J., J. Chem. Eng. Data, (26), 368, 1981

78. Gosting L. J. and Morris, M. S., J.Amer. Chem. Soc, 71, 1998, 1949

79. Pepela C. N., Steel, B. J. and Dunlop, P. J., J. Amer. Chem. Soc., 92, 6743, 1970

80. Lo, H. Y., J. of Chemical Engineering Data, (19) 3, 236, 1974

81. Hayduk $w$. and Loakimidis S., J. of Chemical and Engineering Data, (21) 3, 255, 1976

82. Matthews, M. A. and Akgerman, A., AIChE J., (33)6, 881, 1987

83. Bjorseth, A., Handbook of Polycyclic Aromatic Hydrocarbons, Marcel Dekker, Inc., New York, 1983

84. Wilke, C. R. and Chang, P., AIChE J,, 1, 264, 1955

85. Reddy, K. A. and Doraiswamy, L. K., Ind. Eng. Chem. Fund., $6,77,1966$

86. Lusis, M. A. and Ratcliff, G. A., Can. J. Chem. Eng,, 46, 385,1986

87. King, C. J., Hseuh, L. and Mao, K. W., J. Chem. Eng. Data, $10,348,1965$

88. Reid, R. C., Prausnitz, T. K., Sherwood, T. K., The properties of gases and liquids, 3rd edition, Mcgraw-Hill Book Co., New York, 1977

89. Anderson, J. L., Kathawalla, I. A. and Lindsey, J. S., AIChE Symposium Series $266,(84), 35,1988$

90. Yen, T. F., Amer. Chem. Soc., Div. Pet. Chem. Prep, $24(4), 901,1971$ 
91. Curtis, C. W., Hathaway, D., Guin, J. A. and Tarrer, A. R., Fuel, (9), 575, 1980

92. Bartle, K. D., Mulligan, M. J., Tayler, N., Martin, T. G. and Sanape, C. E., Fuel, (63), 1556, 1984

93. Dark, W. A. and Mcgough, R. R., J. Jf Chromatographic science, (16), 610, 1978

94. Wong, J. L. and Gladstone, C. M., Fuel, (62), 870, 1983

95. Zvans, N., Haley, T. M., Mulligan, M. J., Thomas, K. M., Fuel, (65), 694,1986

96. Lynch, A. W. and Thomas, M. G., Fuel Processing Technology, (8), 13, 1983

97. Baltisberger, R. J., Wagner, S. E., Rao, S. P., Schwan, J.F., and Jones, M. B., Amer. Chem. Soc. Div. Fuel Chem., 29(1), 186, 1984

98. Ruud, A. L., M. S. Thesis, U. of N. Dakoda, 1978

99. Limbach, K. W. and Wei, J.AIChE J., 36(2), 242, 1990

100. Davidson, M. G., Suter, U. W., and Deen, W. M., Macromolecule, 20, 1141, 1987

101. Nourbakhsh, N. Champagnie, A. and Tsotsis, T. T., AIChE Symposiun Series iNo. 268, 85, 75, 1989

102. Nourbakhsh, N. and Tsotsis, T. T., Applied Catalysis, 50, 65, 1989

103. Baltus, R. E., Macromolecule, 22, 1775, 1989

104. Bohrer, M. P., Fetters, L. J., Grizzuti, N., Pearson, D. S., and Tirrell, M. V., Macromolecules, 20, 1827, 1987

105. Davidson, M. G. and Deen, W. M., Macromolecules, 21 , 3474,1988

106. Smith III, F. G. and Deen, W. M., J. of Colloid and Interface Science, $91(2), 571,1983$

107. Smith III, F. G. and Deen, W. M., J. of Colloid and Interface Science, $98(2), 444,1983$

108. SYBYL Molecular Modeling Software Ver. 5.2, Tripos Associates, Inc., St. Louis, Missouri, 1989 
109. Technical product Catalog, Nuclepore Corporation, Pleasanton, California, 1989

110. ASTM F316, American Society for Testing Materials, 1986

111. Stiegel, G. J., Polinski, L. M. and Tischer, R. E., Ind. Eng. Chem. Proc. Des. Dev., 21, 477, 1982

112. Utz, B. R., Narain, N. K., Appell, H. R. and Blaustein B. D., ACS Symposium Series Vol. 205, 225, 1982

113. Narin, N. K., Fuel Processing Techrology, 11, 13, 1985

114. Tischer, R. E., Narain, N. K., Stiegel, G. J. and Cillo, D. L., DOE Technical Report, DOE/PETC/TR-8611, Nov., 1985

115. Smith, J. E., DOE Technical Report, DOE/PC/70814-6, MaY., 1985

116. Nourbakhsh, N. Webster, I. A. and Tsotsis, T. T., $\underline{\text { ACS }}$ Symposiun Series, $33(3), 502,1988$

117. O'Sullivan, J. P. and Woods, G. C., Proc. Roy, Soc. Lond. A, 317, 511, 1970

118. Diggle, J. W., Downie,T. C. and Goulding, C. W., Chem. Res, 69, 365, 1969

119. Woods, G. C. and O'Sullivan, J. P., J.Electrochem Soc, $115,618,1968$

120. Happel, J. and Brenner, H., Low Raynolds Number Hydrodynamics, Martinus Nijhoff, Hague, 1983 as cited in ref. 103

121. Baltus, R. E., Ph.D. Thesis, Carnegie-Mellon University, 1982

122. Yen, T. F., Coal Chemistry Workshop, Aug. 26-27, Monlo Park, California, 1976

123. Sternberg, H. W., Raymond, R. and Schwighardt, F. K. Science, $188,49,1975$

124. Sidney, F. B., MAkovsky, F. L. and schweighardt, F. K., Applied Spectroscopy, 31(3), 241, 1977

125. Rodilosso, P. D. Ph.D. Thesis, University of Pennsylvania, 1984 , as cited in ref. 39

126. Wang, W. P., M.S. Thesis, Auburn University, 1990 


\section{APPENDICES}

218 


\section{APPENDIX A \\ DERIVATION OF THE EQUATION FOR A TRANSPORT OF SOLUTE THROUGH A POROUS MEMBRANE}




\section{Derivation of the Equation for The \\ Porous Membrane Transport}

Consider a membrane transport system shown in Figure A.1. In this system, solute flux across the total membrane area can be expressed as:

$J_{T}=k_{L} A\left(C_{b 1}-\overline{C_{1}}\right)=n A_{D} D_{p}\left(\frac{\overline{C_{1}}-\overline{C_{2}}}{L}\right)=K_{L} A\left(\overline{C_{2}}-C_{b_{2}}\right)$

By eliminating $C_{1}$ and $C_{2}$ from Equation $A .1$, and expressing the equation in terms of flux per unit area of membrane, following equation is obtained:

$$
N_{T}=\frac{C_{b 1}-C_{b 2}}{R_{T}^{0}}
$$

where,

$$
\hat{R}_{T}=\frac{2}{k_{L}}+\frac{1}{e D}
$$

Keller et al. mathematically analyzed the diffusional phenomena of a solute through an ideal porous membrane having discrete identical pores (75). In their analysis,

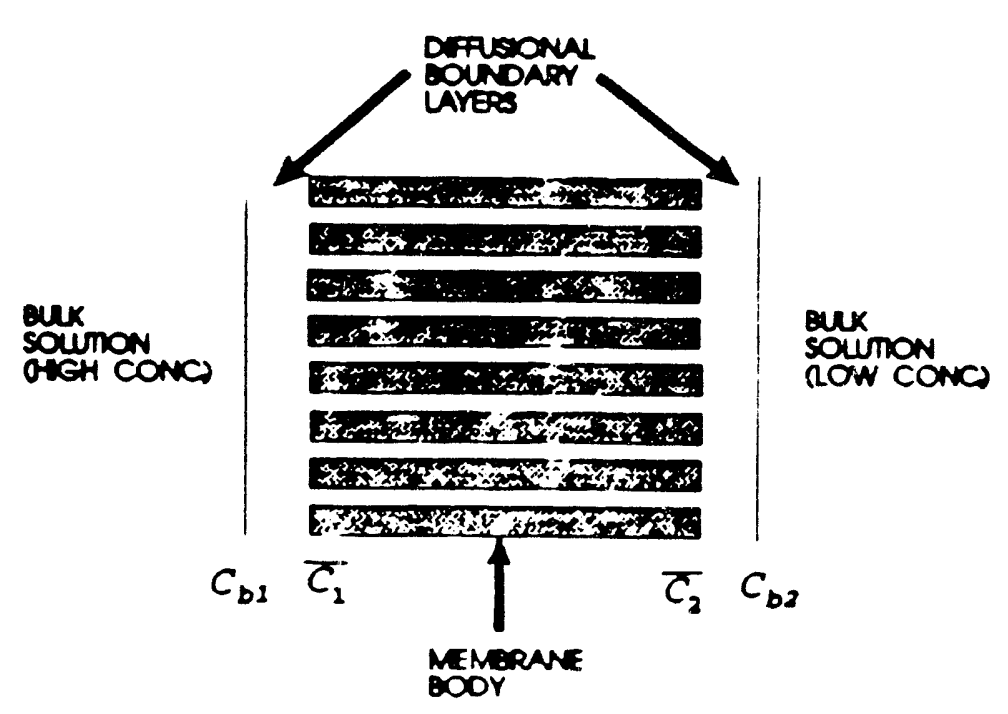

Figure A.1 Drawing for Track-Etched Membrane Transport 
concentration difference of solute across the boundary layer was derived as (Equation (6) in their paper):

$$
\left\langle\overline{C_{1}}-C_{b 1}\right\rangle=\frac{4 q_{i}}{D b^{2}} \sum_{n=0}^{\infty} \frac{J_{1}^{2}\left(\alpha_{m} r_{o}\right)}{\alpha_{m}^{3} J_{0}^{2}\left(\alpha_{m} b\right)} \tanh \left(\alpha_{m} \delta\right)
$$

Here, $q_{i}$ is the solute flux per unit area of pore (i.e., $q_{i}$ $\left.X A_{P}=J_{T}\right), r_{0}$ is the radius of pore, $b$ is the average distance between the axes of adjacent pores, $J_{0}, J_{1}$ are real Bessel functions of the first kind, $\delta$ is the diffusional boundary layer thickness, and the $\alpha_{\mathrm{m}}$ are the roots of an equation, $J_{1}\left(\alpha_{m} b\right)=0$.

Using Equations $A .4$ and A.3, the mass transfer coefficient, $k_{L}$ is obtained as:

$$
k_{L}=\frac{\epsilon D b^{2}}{4 I_{0} \sum_{n=0}^{\infty} \frac{J_{1}^{2}\left(\alpha_{m} r_{0}\right)}{\alpha_{m}^{3} J_{0}^{2}\left(\alpha_{m} b\right)} \tanh \left(\alpha_{m} \delta\right)}
$$

where, $\epsilon$ equals $n \cdot \pi \cdot r_{0}^{2}\left(=n \cdot A_{p} / A\right)$.

Equation $\mathrm{A} .5$ can be simplified by introducing the $\Phi$ function as done by Keller at al.:

$$
k_{L}=\frac{\epsilon D}{4 I_{0} \Phi}
$$

where,

$$
\Phi=\frac{1}{\beta^{2}} \sum_{n=0}^{\infty} \frac{J_{1}^{2}\left(\alpha_{m}^{*}\right)}{\alpha_{m}^{*} J_{0}^{2}\left(\alpha_{m}^{*} \beta\right)} \tanh \left(\alpha_{m}^{*} \Delta\right)
$$

and $\beta=b / r_{0}, \Delta=\delta / r_{0}$, and $\alpha_{m} r_{0}=\alpha_{m}^{*}$.

Then, the total transfer resistance can be expressed as:

$$
R_{T}=\frac{1}{\epsilon D}\left[1+\frac{8 r_{0}}{1} \Phi\right]
$$


Keller et al. reported that the value of $\Phi$ approached an asymptotic value of 0.2 , as $\beta$ increased (75). Based on this observation, Malone et al. (25) derived an approximate equation for the function, $\Phi$, in Equation A.8 by using a curve fitting method. This approximate equation is expressed as:

$$
\Phi=\frac{1}{4}(\epsilon) \frac{\delta}{r_{0}}+\frac{\pi}{16}
$$

The first term on the right-hand side of equation A.9 accounts for the boundary layer resistance while the second term results from the pore entrance and exit effect. The key assumption in Malone et al.'s approach is that the thickness of the boundary layer, $\delta$, is independent of the porosity of membrane, $\epsilon$, and only dependent on the stirring speed. Actually, this hypothesis, that the thickness of boundary layer is not dependent on the porosity of membrane, is inherent in Keller et al.'s model. From Equations A.8 and A.9, Equation 2.2 is obtained as:

$$
R_{T}=\frac{1}{D \cdot \epsilon}+\frac{\pi r_{0} / 2}{D \cdot \epsilon}+\frac{2 \delta}{D}
$$




\section{APPENDIX B}

SAMPLE CALCULATIONS FOR THE ANALYSIS OF

THE EXPERIMENTAL DATA 


\section{Chromatograms of the samples}

In Figure B.1, a chromatogram of the stock solution used in the experiment No. C-R22 is presented, and in Figure B.2. chromatograms of the samples taken from the top compartment and bottom compartment of the diffusion cell after the experiment (Experiment No. C22) are presented. Sample calculations presented in this appendix were made on the basis of these chromatograms.

\section{Sample Calculation for the Effective Diffusivity Measurements of Coal Macromolecules}

Experiment No: Coal_Run 22 .

Date: May, 6, 1990

\section{Experimental Conditions}

- Membrane preparation conditions:

$$
\begin{array}{lc}
\text { Etching time: } & 3.00 \mathrm{~min} \\
\text { Caustic conc.: } & 1.0 \mathrm{~N} \\
\text { Etching temp.: } & 80.0{ }^{\circ} \mathrm{C}
\end{array}
$$

- Average pore diameter determined from the diffusivity measurement of n-octane: $259.0 \AA$ (See next section)

- Diffusion temperature: $25^{\circ} \mathrm{C}$

- Ambient temperature: RMT:= $25^{\circ} \mathrm{C}$

- Stirring speed: $\quad 300 \mathrm{rpm}$

- Diffusion time: $T:=187500 \mathrm{sec}$.

- Total membrane area for diffusion: $S:=7.55 \mathrm{~cm}^{2}$

Inside diameter of gasket: $3.1 \mathrm{~cm}$ 
225

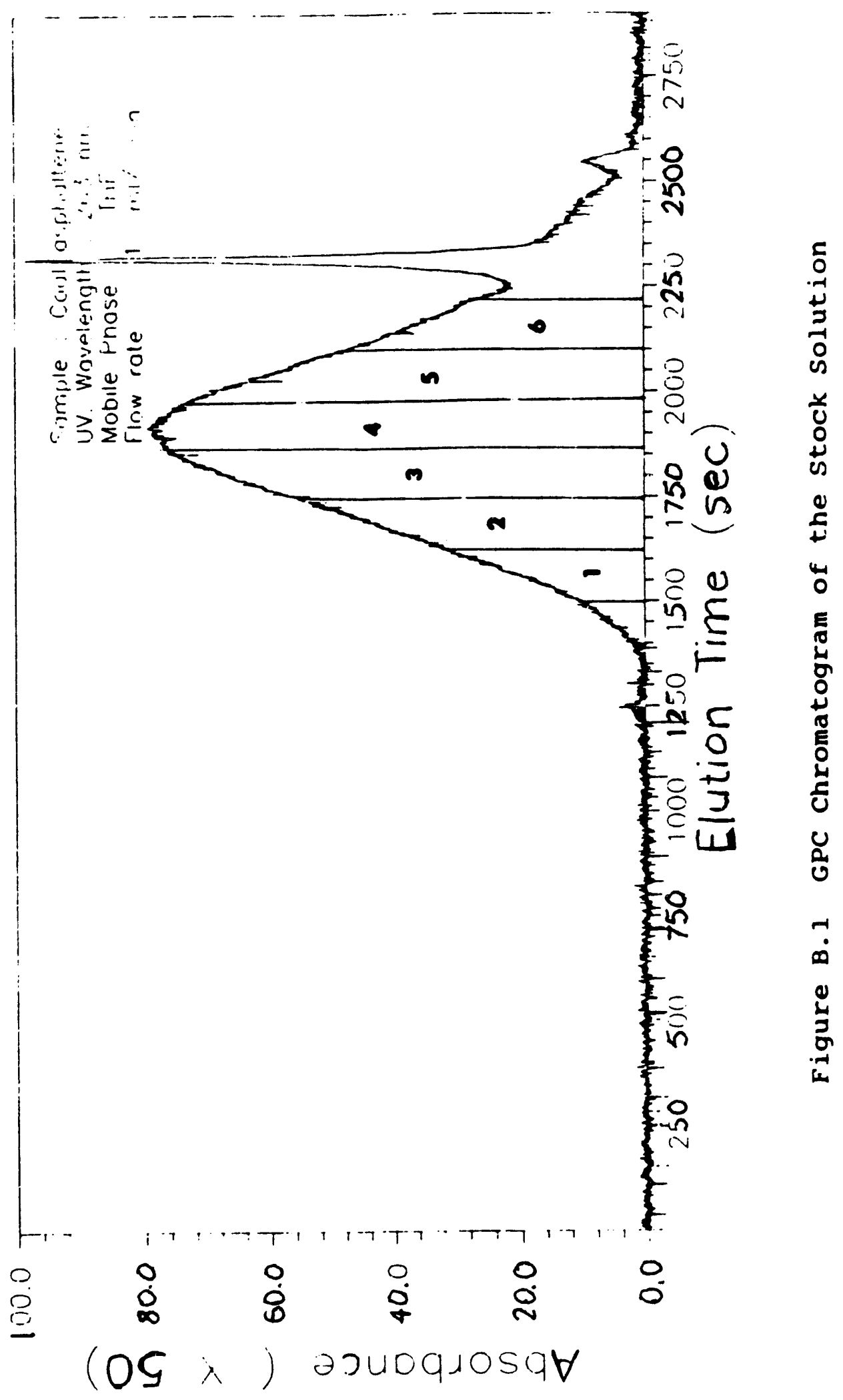




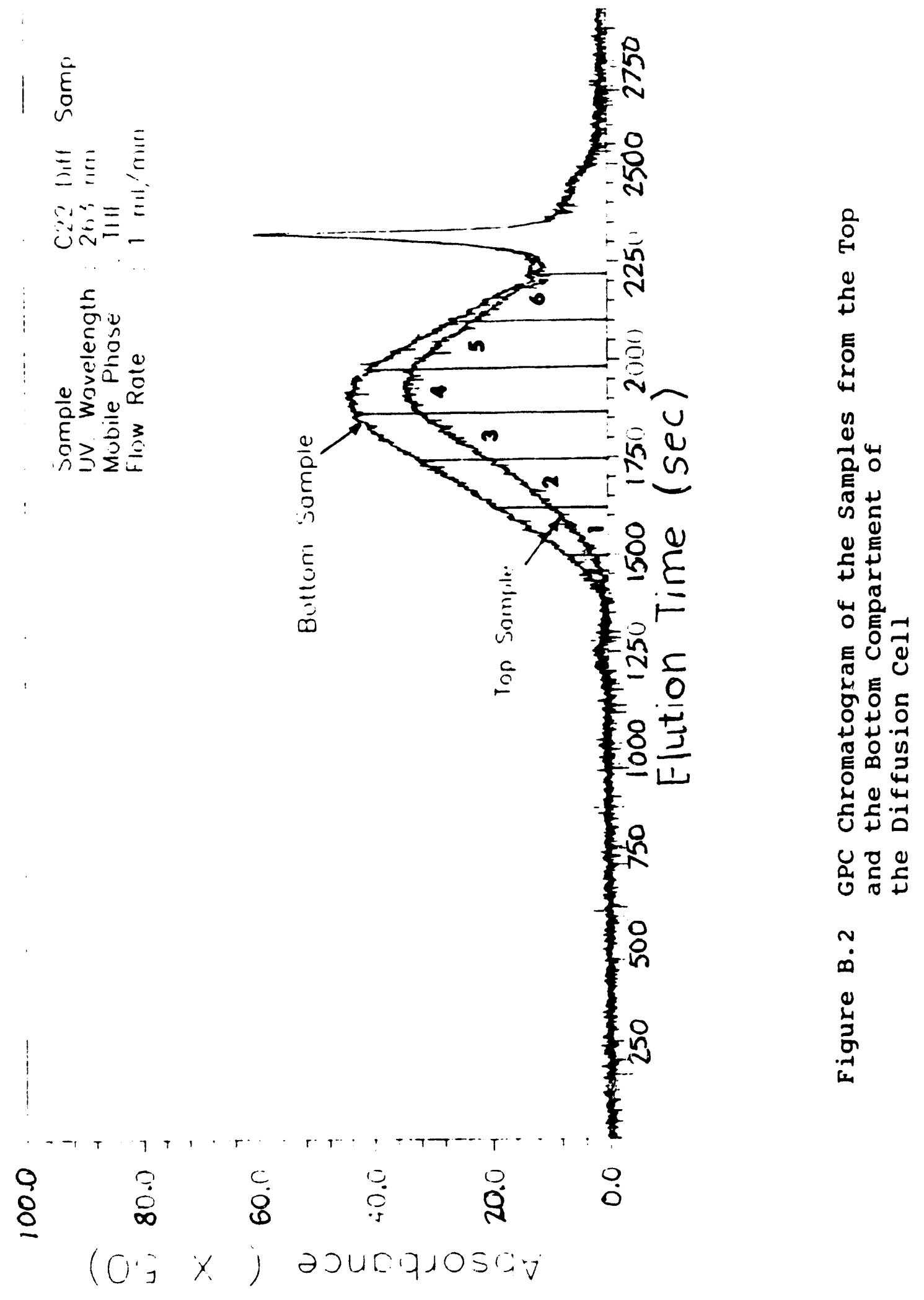




\section{Volume of Each Diffusion Cell Compartment}

- Equation for the density change of ethyl acetate:

Density of ethyl acetate e $20^{\circ} \mathrm{C}: 0.902\left(\mathrm{~g} / \mathrm{cm}^{3}\right)$

Density of ethyl acetate e $25^{\circ} \mathrm{C}$ : $0.895 \quad\left(\mathrm{~g} / \mathrm{cm}^{3}\right)$

DEND: $=0.902-($ RMT -20$)\left[\begin{array}{c}0.902-0.895 \\ -\frac{25-20}{25}\end{array}\right]$

Density of ethyl acetate the current experimental condition: $\quad$ DEND: $=0.895$

- Weight differences

Empty cell weight: EW:= $507.29 \mathrm{gr}$

Cell weight with the bottom

compartment filled: $\quad \mathrm{BW}:=526.62 \mathrm{gr}$

cell weight with the top and

bottom compartment filled: TW: $=543.68 \mathrm{gr}$

- Volume of the bottom compartment

$B V:=\left[\begin{array}{c}B W-E W \\ -D E N D\end{array}\right]$, Bottom volume: $21.61 \mathrm{~cm}^{3}$

- Volume of the top compartment

$$
T V:=\left[\begin{array}{l}
\mathrm{TW}-\mathrm{BW} \\
-\mathrm{DEND}
\end{array}\right], \quad \text { top volume: } 19.07 \mathrm{~cm}^{3}
$$

Areas for coal macromolecules' fractions in chromatogram

- Initial concentration

Frac. \#1: IC $:=2080.9$, Frac. \#2: $I C_{2}:=4577.6$

Frac. \#3: $I C_{3}:=7420.8$, Frac. \#4: $I C_{4}:=8955.4$

Frac. \#5: $I C_{5}:=7233.7$, Frac. \#6: $I C_{6}:=4081.3$ 
- Concentration of top compartment

Frac. \#1: $\mathrm{TC}_{1}:=623.8$, Frac. \#2: $\mathrm{TC}_{2}:=1657.0$

Frac. \#3: $\mathrm{TC}_{3}:=3031.1$, Frac. \#4: $\mathrm{TC}_{4}:=3939.2$

Frac. \#5: $\mathrm{TC}_{5}:=3331.9$, Frac. \#6: TC6:= 1939.9

- Concentration of bottom compartment

Frac \#1: $\quad B_{1}:=1475.8$, Frac. \#2: $\quad B_{2}:=2946.8$

Frac. \#3: $\mathrm{BC}_{3}:=4463.3$, Frac. \#4: $\mathrm{BC}_{6}:=5055.8$

Frac. \#5: $\mathrm{BC}_{5}:=3941.9$, Frac. \#6: $\mathrm{BC}_{6}:=2199.7$

\section{Material balance check}

- Final amount of coal macromolecules

$$
j:=1 \ldots 6
$$$$
\text { FAO }_{j}:=T C_{j} \cdot T V-B C_{j} \cdot B V
$$

$$
\begin{array}{ll}
\mathrm{FAO}_{1}=4.378 \cdot 10^{4} & \mathrm{FAO}_{2}=9.527 \cdot 10^{4} \\
\mathrm{FAO}_{3}=1.542 \cdot 10^{5} & \mathrm{FAO}_{4}=1.843 \cdot 10^{5} \\
\mathrm{FAO}_{5}=1.487 \cdot 10^{5} & \mathrm{FAO}_{6}=8.453 \cdot 10^{4}
\end{array}
$$

- Initial amount of coal macromolecules

$$
\begin{array}{rlrl}
\mathrm{IAO}_{j}:= & I C_{j} \cdot \mathrm{BV} & \\
\mathrm{IAO}_{1} & =4.496 \cdot 10^{4} & \mathrm{IAO}_{2} & =9.890 \cdot 10^{4} \\
\mathrm{IAO}_{3} & =1.603 \cdot 10^{5} & \mathrm{IAO}_{4} & =1.935 \cdot 10^{5} \\
\mathrm{IAO}_{5} & =1.563 \cdot 10^{5} & \mathrm{IAO}_{6} & =8.818 \cdot 10^{6}
\end{array}
$$

- Errors in material balance

$$
\begin{aligned}
& \text { EMB }_{j}:=\frac{\text { IAO }_{j}-\text { FAO }_{j}}{\text { IAO }_{j}} \\
& \mathrm{EMB}_{1}=2.62 \\
& \mathrm{EMB}_{2}=3.68 \\
& \mathrm{EMB}_{3}=3.81 \\
& \mathrm{EMB}_{4}=4.72 \\
& \mathrm{EMB}_{5}=4.85 \\
& \mathrm{EMB}_{6}=4.15
\end{aligned}
$$


Total resistance calculation

- From equation 2.1, (unit ; sec/cm)

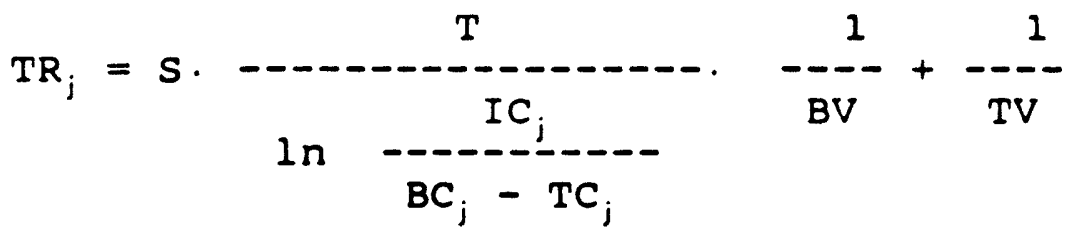

$$
\begin{aligned}
& \mathrm{TR}_{1}=1.565 \cdot 10^{5} \quad \mathrm{TR}_{2}=1.103 \cdot 10^{5} \\
& T R_{3}=8.493 \cdot 10^{4} \quad T R_{4}=6.710 \cdot 10^{4} \\
& \mathrm{TR}_{5}=5.649 .10^{4} \quad T R_{6}=5.072 \cdot 10^{4}
\end{aligned}
$$

Calculation of effective diffusion coefficients

- Average pore radius: $r:=129.5 \cdot 10^{-8} \mathrm{~cm}$

- Pore length: $1:=6 \cdot 10^{-6} \mathrm{~cm}$

- Pore density per one $\mathrm{cm}^{2}: \mathrm{n}:=3 \cdot 10^{8}$

- From equation 2.2, (neglect the boundary layer resistance and the pore entrance and exit effect.), ( unit: $\mathrm{cm}^{2} / \mathrm{sec}$ )

$$
\begin{array}{rlr}
\text { Deff } f_{j}= & =\frac{1}{T R_{j} \cdot n \cdot \pi \cdot r^{2}} & \\
\text { Deff } f_{1}=2.425 \cdot 10^{-6} & \operatorname{Deff}_{2}=3.434 \cdot 10^{-6} \\
\operatorname{Deff}_{3}=4.469 \cdot 10^{-6} & \operatorname{Deff}_{4}=5.657 \cdot 10^{-6} \\
\operatorname{Deff}_{5}=6.720 \cdot 10^{-6} & \text { Deff }_{6}=7.485 \cdot 10^{-6}
\end{array}
$$

\section{Calculation of a Membrane's Pore size from Diffusivity Measurement of n-octane}

Experiment No.: Coal_Run 22

- Average pore size of membrane used in the previous calculation for the analysis of experimental data was determined as follows. 
- Total transfer resistance $\left(R_{T}\right)$ obtained from a in situ diffusivity measurement of n-octane: 17400 (sec/cm)

- Equation 3.5 :

$$
\frac{1 / n \pi r_{0}^{2}}{D_{-}\left[1+\left(\frac{9}{8}\right)\left(\frac{a}{r_{0}}\right) \ln \left(\frac{a}{I_{0}}\right)-1.54\left(\frac{a}{I_{0}}\right)\right]}=R_{T}
$$

- Use Stokes Einstein radius of n-octane $\left(r_{s}\right)$ instead of 'a', a radius of model sphere.

- Diffusivity of n-octane in ethyl acetate $\left(D_{0}\right)$ :

$$
2.47 \times 10^{-5} \mathrm{~cm}^{2} / \mathrm{sec} \text { (from chapter VI) }
$$

- Viscosity of ethyl acetate at $25^{\circ} \mathrm{C}(\mu): 0.441$ (cp) Density of ethyl acetate at $25{ }^{\circ} \mathrm{C}(\rho): 0.894\left(\mathrm{~cm}^{3} / \mathrm{g}\right)$

From CRC Handbook of Chemistry and Physics (64th ed.)

- from Stokes-Einstein equation,

$$
\begin{aligned}
I_{s} & =\frac{k T}{6 \pi \mu D_{-}} \\
& =\frac{\left(1.38 \times 10^{-16}\right)(298)\left(1 \times 10^{8}\right)}{(6 \pi)\left(0.441 \times 10^{-2}\right)\left(2.47 \times 10^{-5}\right)} \\
& =2.00 \mathrm{~A}
\end{aligned}
$$

- Calculation of the boundary layer resistance $\left(R_{b}\right)$ Empirical equation developed in the current study: $\mathrm{N}_{\text {sh }}=0.084 \mathrm{~N}_{\mathrm{Re}}^{0.68} \mathrm{~N}_{\text {sc }}{ }^{0.33}$ Reynolds number $\left(N_{R e}\right)$ is expressed as $:\left(\omega \cdot d^{2} \cdot \rho / \mu\right)$

$$
\begin{aligned}
& \text { Argular velocity } \begin{aligned}
(\omega) & =(2 \pi \mathrm{rpm}) / 60 \\
& =2 \pi \times 300 / 60=31.4
\end{aligned} \\
& \begin{aligned}
\mathrm{N}_{\mathrm{Re}}=31.4 \times \mathrm{d}^{2} \times 0.894 / 0.441 \times 10^{.2},(d=3.1) \\
=61172 \text { (using } \mathrm{d} \text { as inside diameter of gasket) }
\end{aligned}
\end{aligned}
$$




$$
\begin{aligned}
N_{\text {Sc }}=\mu / \rho / D_{0}=0.441 \times 10^{-2} / 0.894 / 2.45 \times 10^{-5} \\
=201.34
\end{aligned}
$$

From the empirical equation, Sherwood numbers $\left(\mathrm{N}_{\mathrm{Sh}}\right)$

is determined as:

$$
\begin{aligned}
N_{\text {Sh }}= & 0.084 \times(61171)^{0.68} \times(201.34)^{0.33} \\
& =885.35
\end{aligned}
$$

From the definition of sherwood numbers $\left(d / R_{b} D_{0}\right)$

$R_{b}=3.1 /\left(885.35 \times 2.45 \times 10^{-5}\right)=142.91(\mathrm{sec} / \mathrm{cm})$

- As shown in this sample calculation, the boundary layer resistance was negligible $(1.6 \%$ of the total transfer resistance) in comparison with the membrane resistance, thus, in Equation 3.5, the total transfer resistance term $\left(R_{t}\right)$ was used instead of the membrane resistance. However, In the actual calculation of pore diameters, the boundary layer term was not neglected.

- Pore radius calculated from a root-finding method:

$$
r_{0}=129.5 \AA
$$

- Right hand side of Equation 3.5:

$$
\begin{aligned}
R_{m} & =R_{t}-2 \times R_{b} \\
& =17400-2 \times 142.9=17114.2(\mathrm{sec} / \mathrm{cm})
\end{aligned}
$$

- Left hand side of Equation 3.5:

$$
\begin{aligned}
& \text { Hinderance factor } f(\lambda): \lambda=r_{s} / r_{0}=0.0155 \\
& \begin{aligned}
f(\lambda) & =1+(9 / 8)(0.0155) \ln (0.0155)-(1.539)(0.0155) \\
& =0.09108
\end{aligned}
\end{aligned}
$$

Membrane properties are already given $\left(1, n, r_{0}\right)$ 
- Left side $=\left(6 \times 10^{-4}\right) /\left(3 \times 10^{8}\right) / \pi /(129.5 \times 129.5)$

$$
\begin{aligned}
& \times 10^{-16} / 2.45 \times 10^{-5} / 0.9395 \\
= & 17011 \approx 17114=\text { Right side }
\end{aligned}
$$

- Thus, the pore size obtained from a root finding method was a correct one. Difference between the right hand side value and the left hand side value appeared to be caused by the round-off errors during the root-finding process, since the logarithm term is involved in the hinderance factor.

\section{Analysis of Experimental Data Obtained from Indirect Measurement Methods}

Experimental data obtained from indirect measurement methods were analyzed using Equation 6.2. Here, a sample calculation for the indirect measurement of naphthalene's diffusivity using the diffusivity of n-octane is presented.

- Equation 6.2 is expressed as:

$$
\frac{D_{a_{2}}}{D_{a_{2}}}=\frac{R_{T_{2}}-2 R_{b_{2}}}{R_{T_{1}}-2 R_{b_{1}}} \cdot \frac{f\left(\lambda_{2}\right)}{f\left(\lambda_{1}\right)}
$$

where

$$
f\left(\lambda_{i}\right)-1+\left(\frac{9}{8}\right)\left(\lambda_{i}\right) \ln \left(\lambda_{i}\right)-1.539 \lambda_{i}, \quad \lambda_{i}-\frac{I_{s_{1}}}{I_{0}}
$$

- Total transfer resistance $\left(R_{T}\right)$ obtained from a in situ diffusivity measurement of n-octane: 27569 (sec/cm)

- Total transfer resistance $\left(R_{12}\right)$ obtained from a diffusivity measurement of Naphthalene: 28094 ( $\mathrm{sec} / \mathrm{cm}$ ) 
$2.43 \times 10^{-5} \mathrm{~cm}^{2} / \mathrm{sec}$.

- Rearranging Equation 6.2 as:

$$
D_{a_{1}} \cdot\left(R_{T_{1}}-2 R_{b_{1}}\right) \cdot f\left(\lambda_{1}\right)=D_{a_{2}} \cdot\left(R_{T_{1}}-2 R_{b_{2}}\right) \cdot f\left(\lambda_{2}\right)
$$

- Calculation of the boundary layer resistance $\left(R_{b 1}\right)$

Empirical equation developed in the current

study: $\quad \mathrm{N}_{\text {Sh }}=0.084 \mathrm{~N}_{\text {Re }}^{0.68} \mathrm{~N}_{\text {Sc }}^{0.33}$

Reynolds number $\left(N_{R_{e}}\right)$ is expressed as : $\left(\omega \cdot d^{2} \cdot p / \mu\right)$

Angular velocity $(\omega)=(2 \pi \mathrm{rpm}) / 60$

$$
=2 \pi \times 300 / 60=31.4
$$

$N_{R e}=31.4 \times d^{2} \times 0.894 / 0.441 \times 10^{-2},(d=3.1)$

$=61202$ (using $d$ as inside diameter of gasket)

$N_{s c 1}=\mu / P / D_{0}=0.441 \times 10^{-2} / 0.894 / 2.47 \times 10^{-5}$

$=199.71$

From the empirical equation, sherwood numbers $\left(\mathrm{N}_{\mathrm{sh}}\right)$

is determined as:

$$
\begin{aligned}
\mathrm{N}_{\text {sh } 1}= & 0.084 \times(61202)^{0.68} \times(199.71)^{0.33} \\
& =883.254
\end{aligned}
$$

From the definition of Sherwood numbers $\left(d / R_{b} D_{0}\right)$

$$
R_{b 1}=3.1 /\left(883.25 \times 2.47 \times 10^{-5}\right)=142.91(\mathrm{sec} / \mathrm{cm}) \cdot
$$

- Calculation of the boundary layer resistance $\left(R_{b 2}\right)$

$$
\begin{aligned}
N_{R e} & =61202 \text { (same as the one used previously) } \\
N_{\text {sc2 }} & =\mu / \rho / D_{02}=0.441 \times 10^{-2} / 0.894 / 2.43 \times 10^{.5} \\
& =202.978
\end{aligned}
$$

Sherwood numbers $\left(\mathrm{N}_{S h}\right)$ is determined as:

$N_{\text {Sh2 }}=0.084 \times(61202)^{0.68} \times(202.978)^{0.33}$ 
Sherwood numbers $\left(\mathrm{N}_{S h}\right)$ is determined as:

$$
\begin{aligned}
N_{\text {Sh2 }}= & 0.084 \times(61202)^{0.68} \times(202.978)^{0.33} \\
& =888.04
\end{aligned}
$$

From the definition of sherwood numbers $\left(d / R_{b} D_{0}\right)$ $R_{b 2}=3.1 /\left(888.04 \times 2.34 \times 10^{-5}\right)=143.64(\mathrm{sec} / \mathrm{cm})$.

- Hinderance factor $f\left(\lambda_{1}\right)$ :

$$
\begin{aligned}
\lambda_{1} & =r_{s 1} / r_{0}=2.0 / 103.594=0.0193 \\
f\left(\lambda_{1}\right) & =1+(9 / 8)(0.0193) \ln (0.0193)-(1.539)(0.0193) \\
& =0.8844
\end{aligned}
$$

- Hinderance factor $f\left(\lambda_{2}\right)$ :

from stokes-Einstein equation,

$$
\begin{aligned}
& r_{s}=\frac{k T}{6 \pi \mu D_{-}} \\
& =\frac{\left(1.38 \times 10^{-16}\right)(298)\left(1 \times 10^{8}\right)}{(6 \pi)\left(0.441 \times 10^{-2}\right)\left(2.43 \times 10^{-5}\right)} \\
& =2.035 \AA \\
& \lambda_{2}=r_{s 2} / r_{0}=2.035 / 103.594=0.01965 \\
& f\left(\lambda_{2}\right)=1+(9 / 8)(0.01965) \ln (0.01965)-(1.539)(0.01965) \\
& =0.8829
\end{aligned}
$$

- Left side:

$$
(27596-2 \times 142.91) \times 0.8844 \times 2.47=59658
$$

- Right side:

$$
(28093-2 \times 146.64) \times 0.8829 \times 2.43=59643
$$

- Thus, the solution of the nonlinear equation (the diffusiviy of naphthalene) is proved to be correct. 


\section{APPENDIX C \\ SOLUTE FLUX THROUGH A POLYCARBONATE MEMBRANE MATRIX}




\section{Solute Flux Through Polycarbonate Membrane Matrix}

As previously noted in Chapter III, solute flux through a membrane matrix (not membrane pores) was observed in the etching study of polycarbonate film. Since polycarbonate membranes were adopted in the previous hindered diffusion studies where ethyl acetate was used as a solvent $(30,47)$, this observation, the flux through membrane matrix, appeared to have some effect on the results reported in the literature. Details of the experimental results regarding the flux observation and the results of Fourier Transform Infra-Red spectrometer (FTIR) study to elucidate the observation are presented here.

\section{Experiment}

Polycarbonate membranes were prepared under the same experimental conditions used in previous etching experiments, and diffusivity measurements with n-octane in ethyl acetate were conducted to determine the pore sizes of the membranes produced from the etching processes.

\section{Results and Discussion}

Etching curves: In Figure C.1, the etching curve determined from the diffusivity measurements of n-octane in ethyl acetate is presented. The diffusivity of n-octane in ethyl acetate used in this calculation was $2.47 \times 10^{-5}$ $\mathrm{cm}^{2} / \mathrm{sec}$ as reported in chaper IV. As shown in this figure, 
the etching curve obtained from the diffusion experiments using ethyl acetate does not agree with the etching curve from the previous study. Higher n-octane fluxes were observed in the diffusion experiments with ethyl acetate. In this figure, the polycarbonate membrane even shows some degree of permeation of n-octane in the diffusion experiments with a membrane having no pores (no etching). The calculated pore size increases linearly as the etching time increases after a certain incubation time. This incubation time is close to the pore opening time observed in the diffusion experiments with $n$-decane. These observations may be attributed to the fact that ethyl acetate interacts with the polycarbonate membrane (polar interactions).

FTIR study: To elucidate the observation in Figure C.1, FTIR spectra (with a Nicolet model 5SXC) were taken for the treated and untreated polycarbonate films with ethyl acetate. The treatment of polycarbonate film was conducted as follows: by dipping the polycarbonate film in ethyl acetate about 10 minutes and drying the membrane in a vacuum oven at $60^{\circ} \mathrm{C}$ about an hour. In Figures $\mathrm{C} .2$ and $\mathrm{C} .3$, FTIR spectra of the untreated and treated films are presented. The major differences between the two spectra can be found around the wave number 1770, which corresponds to the $c=0$ band spectrum of the polycarbonate film. In the FTIR spectrum of treated polycarbonate $f i l m$, this $C=0$ band peak 


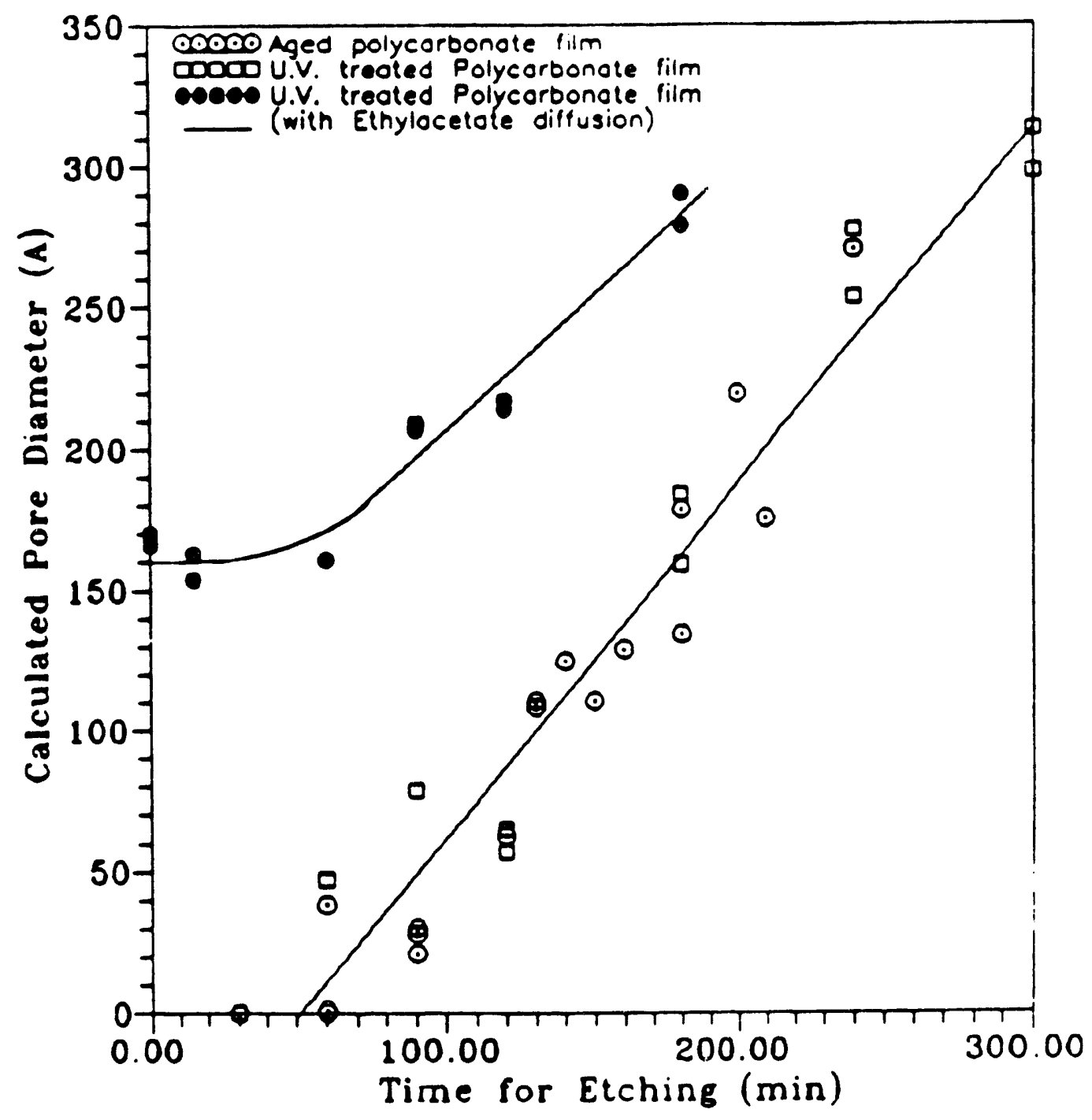

Figure C.1 Etching Curves of Polycarbonate Films Determined from $C_{8} /$ Ethyl Acetate system. 
is shifted a little, and a new peak with wave number 1736 is observed. This new peak comes from the $\mathrm{C}=0$ band spectrum of ethyl acetate. The new peak is an evidence that ethyl acetate was bound within the polycarbonate membrane matrix in the treated membrane. It is not clear whether this binding of ethyl acetate causes structural changes in polycarbonate film. However, it is possible to surmise a structural change of polycarbonate film if the general structural property of polymers and the film making process are considered.

Effect on the hindered diffusion study: In the previous hindered diffusion study with polycarbonate membranes in ethyl acetate, performed by Guilliot and Cannel et al., the molecular sizes and pore sizes of the membranes used in the hindered diffusion study were fairly large, and so the solute flux through the membrane matrix was neglected $(30,47)$. For the hindered diffusion study of relatively small molecules such as coal asphaltene, smaller pore sizes of membranes (at least less than $150 \AA$ ) are required to conduct the hindered diffusion study more effectively. However, for the membrane having these pore size ranges, the solute flux through the membrane matrix is significant compared to the overall flux across the membrane as shown in Figure C.1. Also, the pore diffusion should be isolated from the overall membrane diffusion in order to study the hindered diffusional phenomena. Thus, polycarbonate 


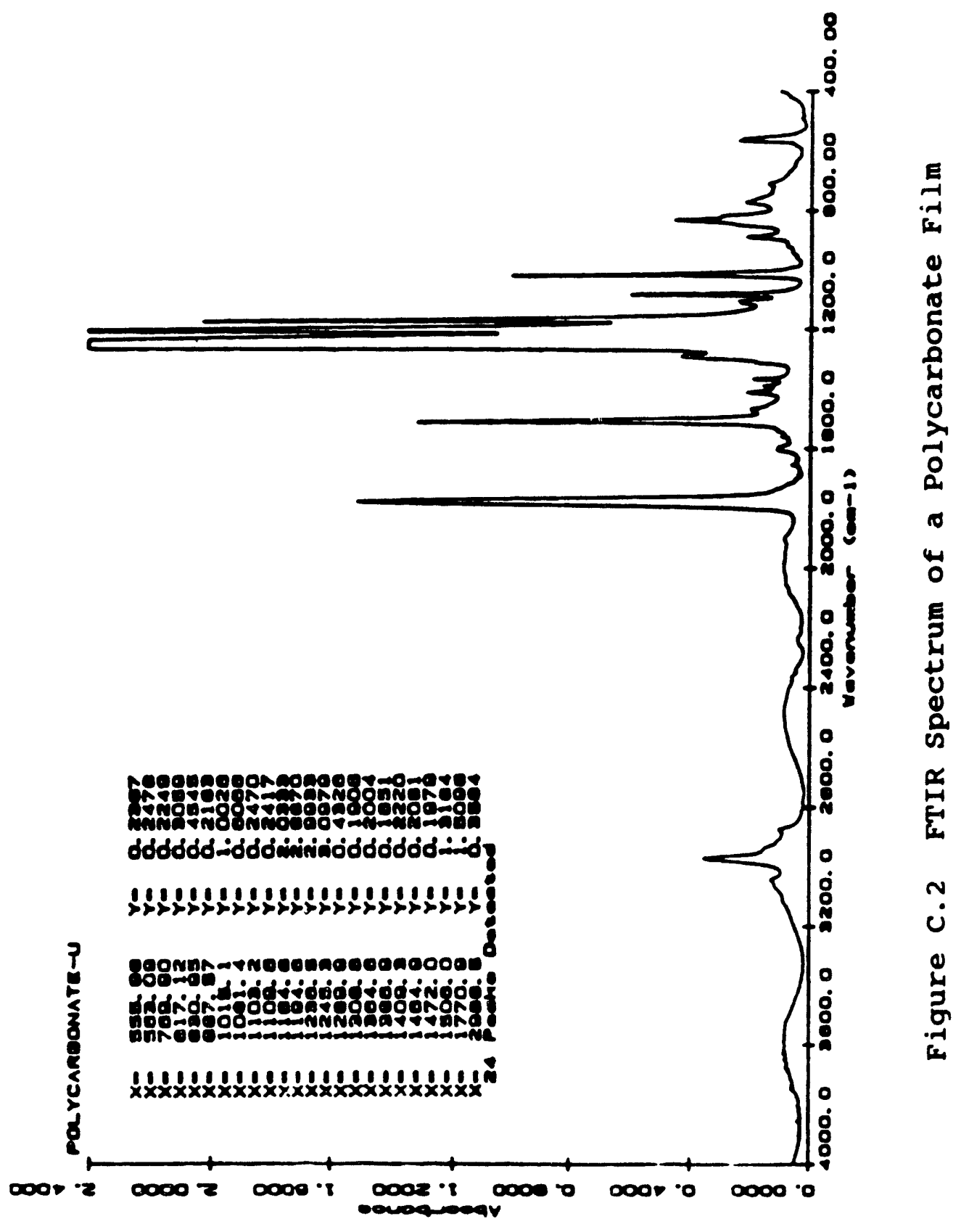




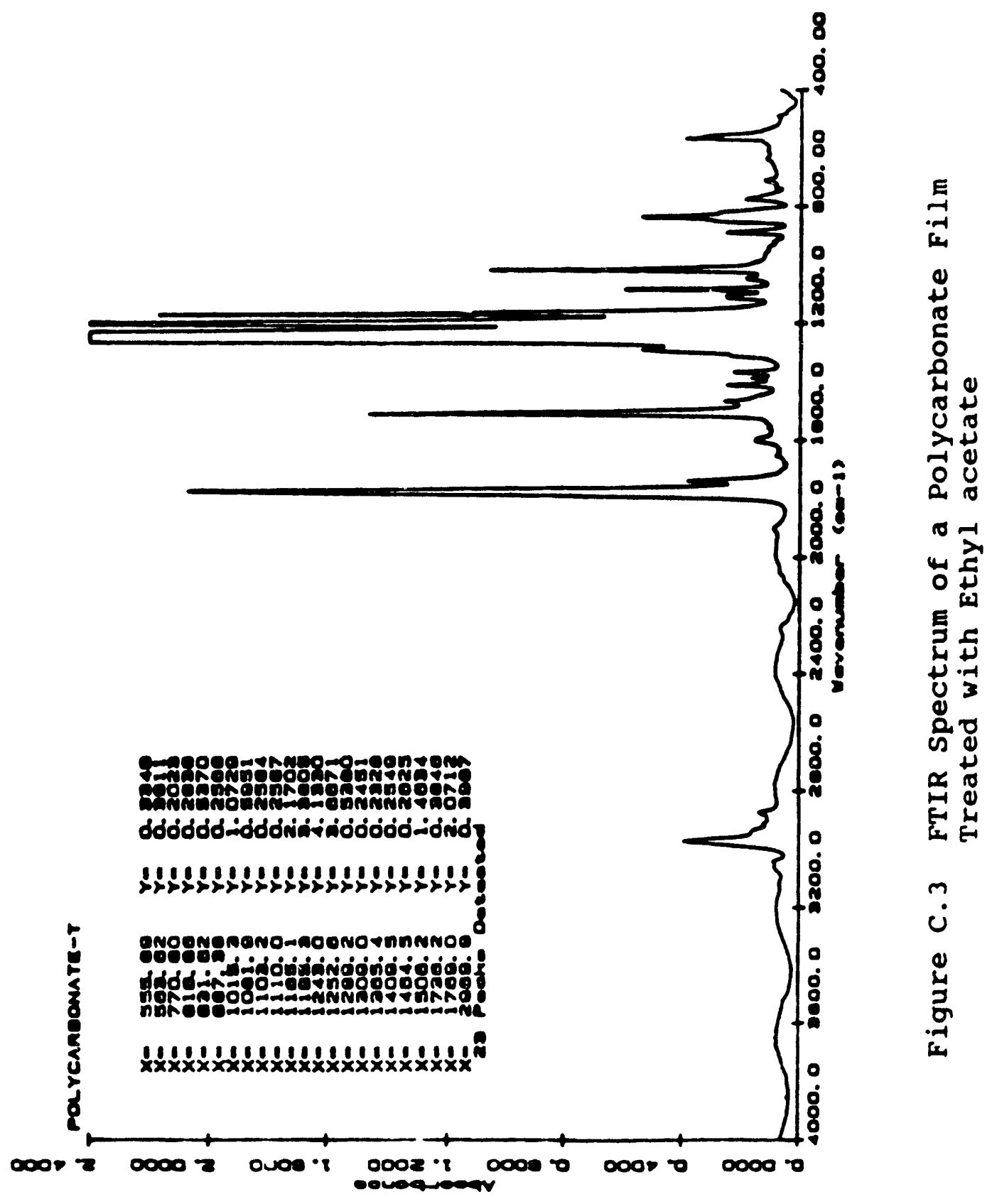


membranes were not adopted in a hindered diffusion experiment conducted in the current research. 


\section{APPENDIX D \\ ESTIMATION OF THE DIFFUSION COEFFICIENT OF \\ N-OCTANE IN N-DECANE}




\section{Estimation of The Diffusion Coefficient of} N-Octane's in $\mathrm{N}$-Decane

\section{A Correlation Equation Proposed by Lo}

Lo (80) proposed following equation for the estimation of diffusion coefficient in n-binary alkane system. The equation correlates the diffusivity of $n$-paraffin in terms of the number carbon atoms in solute $\left(n_{1}, n_{2}\right)$ and the self diffusion coefficient $\left(D_{22}\right)$ of solvent.

$$
D_{12}-D_{22}+\frac{\log \left(\frac{n_{1}}{n_{2}}\right)}{\left(0.1964-0.06785 n_{2}\right)} \times 10^{-5}
$$

Average percentage difference between the experimental values and the estimated values from this equation was calculated to be 4.2 (Table II in Lo's paper (80)).

The experimental value of n-octane's diffusivity in $n$ decane was not given in his paper. However, the experimental value for the diffusivity of $n$-heptane in $n$ decane was measured in his work and was reported to be 1.73 $x 10^{-5} \mathrm{~cm}^{2} / \mathrm{sec}$. The author reported that his equation underestimated this experimental value by $5.5 \%$. Thus it was eypected that the diffusivity of $n$-octane in $n$-decane would be lower than this value.

\section{A Correlation Equation Proposed by Hayduk}

Hayduk et al.(81) observed that diffusivities of a solute dissolved in a number of different solvents had a certain relationship with the viscosities of solvents in the 
absence of strong molecular interactions and proposed the following correlation:

$$
D_{12}=A^{-B}
$$

They applied this equation to n-paraffinic hydrocarbon systems and constructed a viscosity-diffusivity map in their study (Figure 5 in Hayduk et al's paper). Most paraffinic hydrocarbon diffusivity data fell in straight lines as seen in their figure.

Based on this observation, parameters $(A, B)$ associated with n-octane's diffusivity and n-dodecane's diffusivity were obtained using experimental data shown in Table D.2. Resulting equations are;

$$
D=1.46 \mu^{-0.70} \text { for n-octane }
$$

and

$$
D=1.10 \mu^{-0.73} \text { for } n \text {-dodecane }
$$

\section{Evaluation of the Estimation Methods}

To evaluate Lo's equation and Hayduk et al.'s method in predicting diffusivity data, a comparison table for diffusivities of several n-paraffins is provided. Self diffusivity data used in the Lo's correlation are obtained from Hayduk et al's paper (Table II). As shown in Table D.2, both methods predict the diffusivity values very accurately. Thus, an average of the predicted values from these two methods for n-octane diffusivity $12.62 \times 10^{-5}$ $\mathrm{cm}^{2} / \mathrm{sec}$ ) was used in the current study. 
Table D. 1

Sources of Diffusivity Data e $25^{\circ} \mathrm{C}$ Used for the Calculation

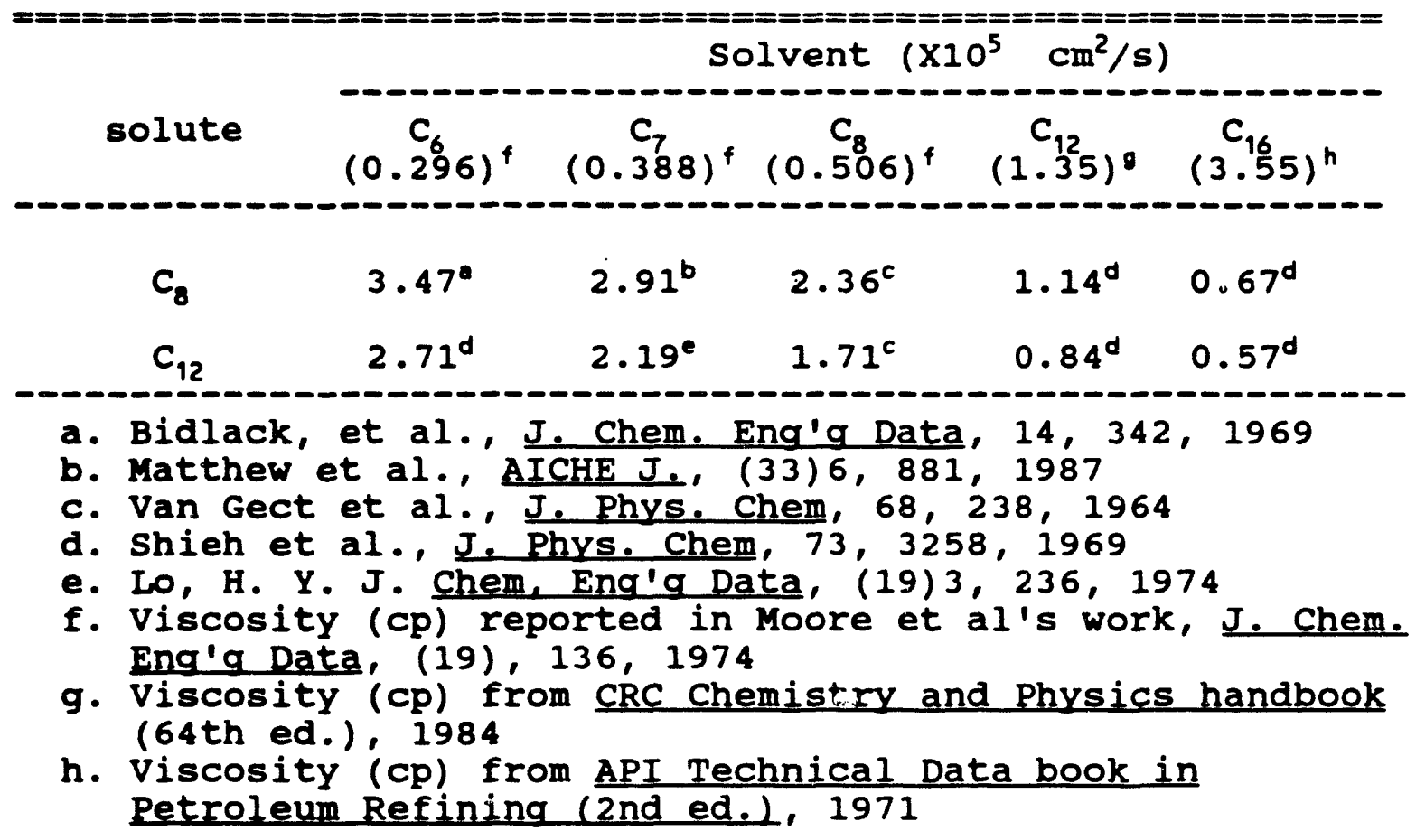


Table D.2

Comparison of Methods for the Estimation of Some Paraffinic Hydrocarbons'

Diffusivities

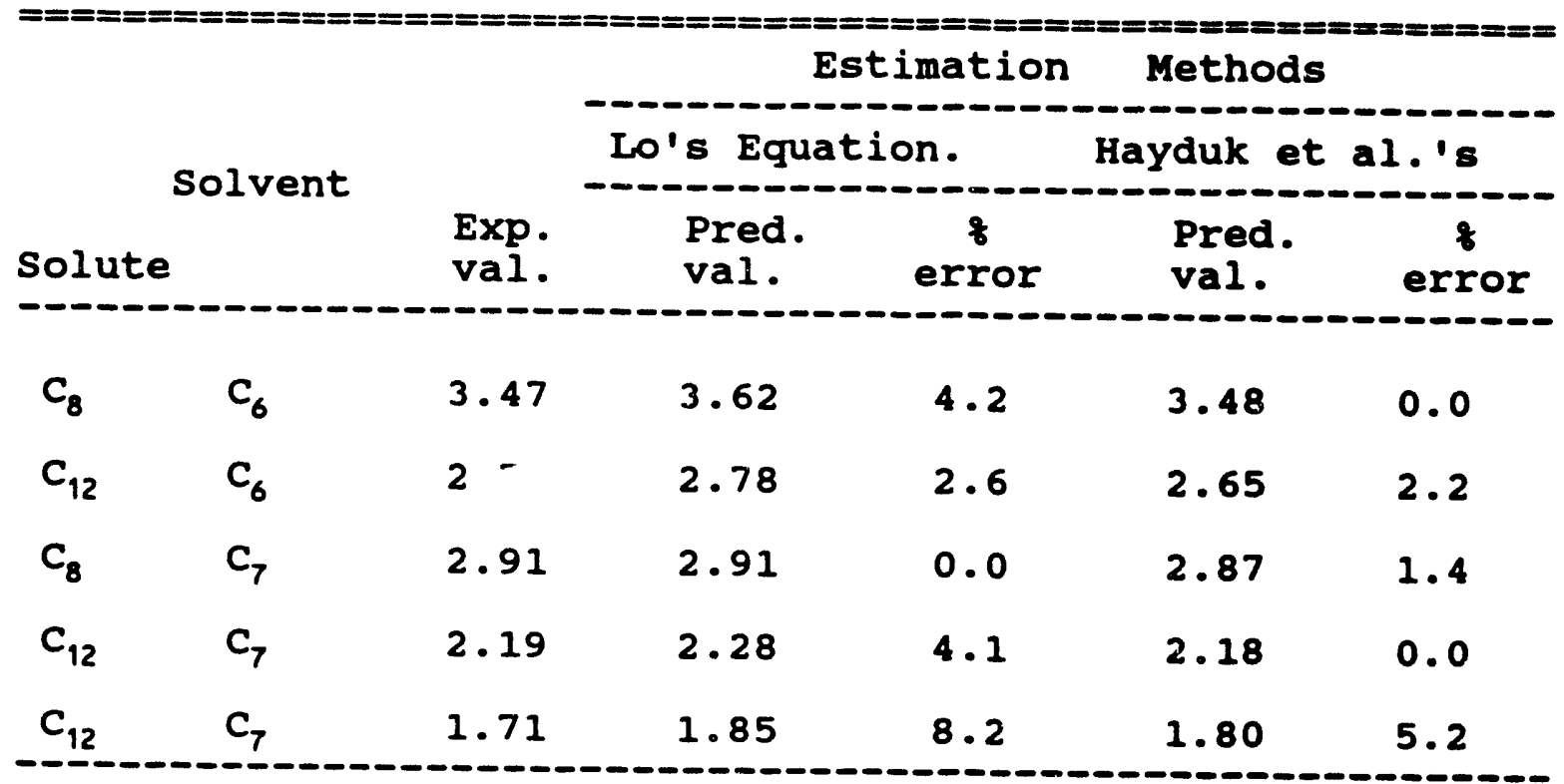


APPENDIX E

THEORETICAL CURVES FOR THE HINDRANCE FACTOR 


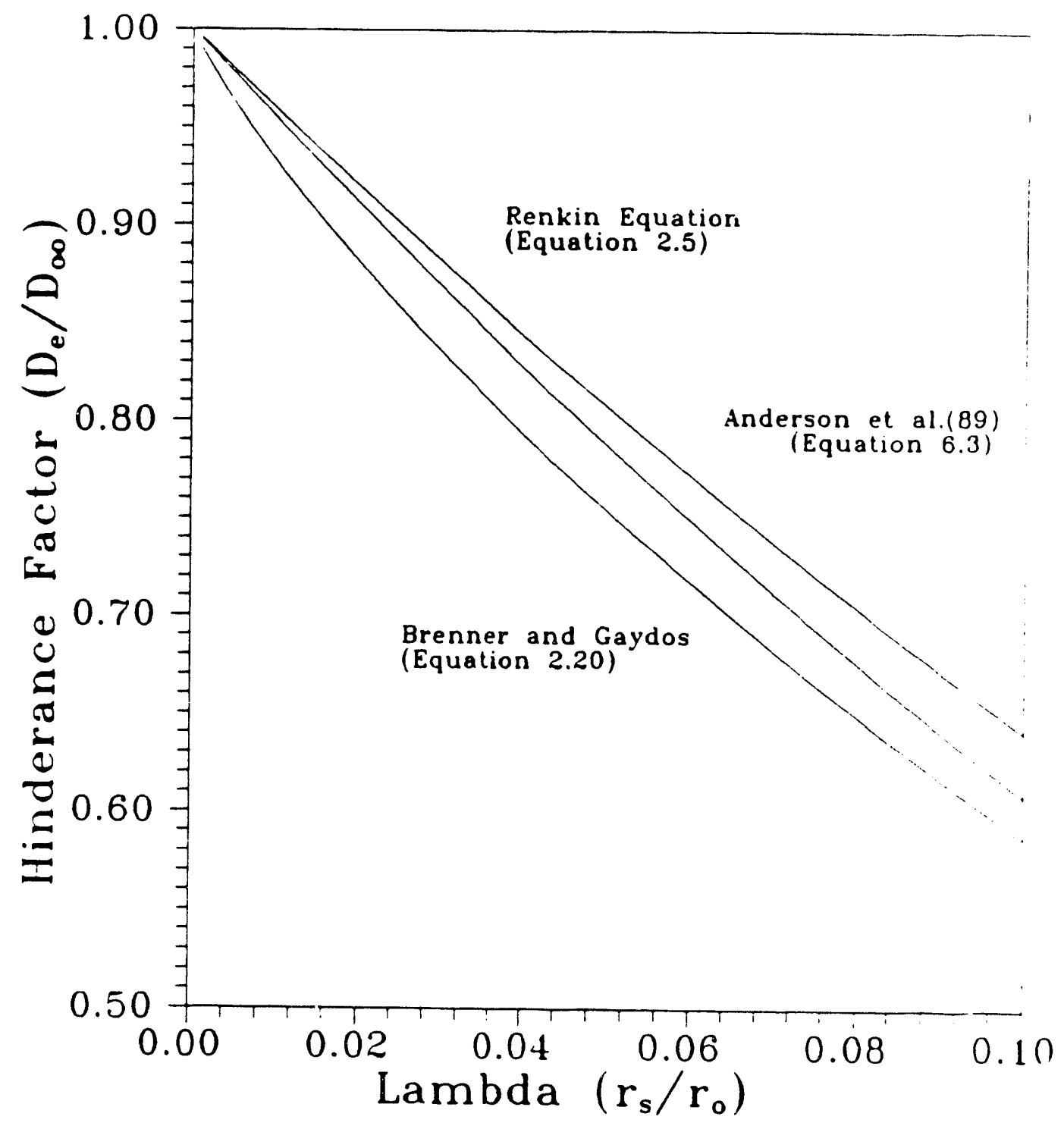

Figure E.1 Theoretical Curves for the Hindrance Factor 
APPENDIX F

ERROR ANALYSIS

250 


\section{Error Analysis}

An evaluation of experimental factors influencing the uncertainty of diffusivity data obtained in the current research is provided in this appendix. In general, for a variable $Y$ which may be expressed as a function of $n$ variables, $x_{1}, x_{2}, \ldots x_{n}$, the uncertainty in $Y$ was obtained by:

$$
S^{2}(Y)-\sum_{i=1}^{n}\left(\frac{\partial Y}{\partial x_{i}}\right)^{2} S^{2}\left(x_{i}\right)+\ldots
$$

Here, $\mathbf{S}$ stands for the standard deviation of each variable. The uncertainty of diffusivity data obtained in the current research are dependent on many experimental variables as seen in Equation 2.1 and 2.2. Basically, all experimental variables are subject to error analysis in order to evaluate the uncertainty of diffusivity data. However, in this error analysis section, special interest was given to the variables associated with the properties of track-etched membranes and the concentration analysis, since these were the major sources of error in the current research.

\section{Effect of Errors in Pore Length}

In the analyses of experimental data used in the current study, nominal values of membrane thicknesses supplied by manufacturer were used for actual pore lengths (1). However, the maximum deviation of the actual pore lengths from the nominal membrane thicknesses reported in the literature (26) was $6.8 \%$, as described in Chapter III. 
since this type of error is not random, all error analyses conducted in this section were based on this maximum value. The errors in pore length affect the errors in pore size determination from permeability tests and diffusivity measurements of $n$-octane and, consequently, influence the errors in diffusivity data. In Table F.1, results of error analyses in the pore size determination methods are summarized, and in Table F.2, the effects of errors in pore lengths on the diffusivity data of TPP and coal macromolecules are summarized. In the same table, relative erzors in the ratios of effective to bulk diffusivities and in the ratios of stokes-Einstein radii to pore radii affected by the errors in pore length are also summarized. As shown in these tables, the relative errors in these ratios are not significant. Thus, it appears that the errors in pore length do not seriously affect the positions of experimental data presented in Figures 6.5 and 7.5 . In other words, errors in pore lengths are not primarily reponsible for the differences between experimental data and theoretical curves observed in Figures 6.5 and 7.5 .

Errors in Pore densities of Membranes Errors in pore densities affect the errors in measured pore size data and diffusivity data in a manner similar to the errors in pore length. In this analysis, the tolerance limit of rated pore densities specified in Nuclepore 
Table F.1

Effects of Errors in Pore Length on Uncertainties of Pore Sizes Determined from the Various Methods

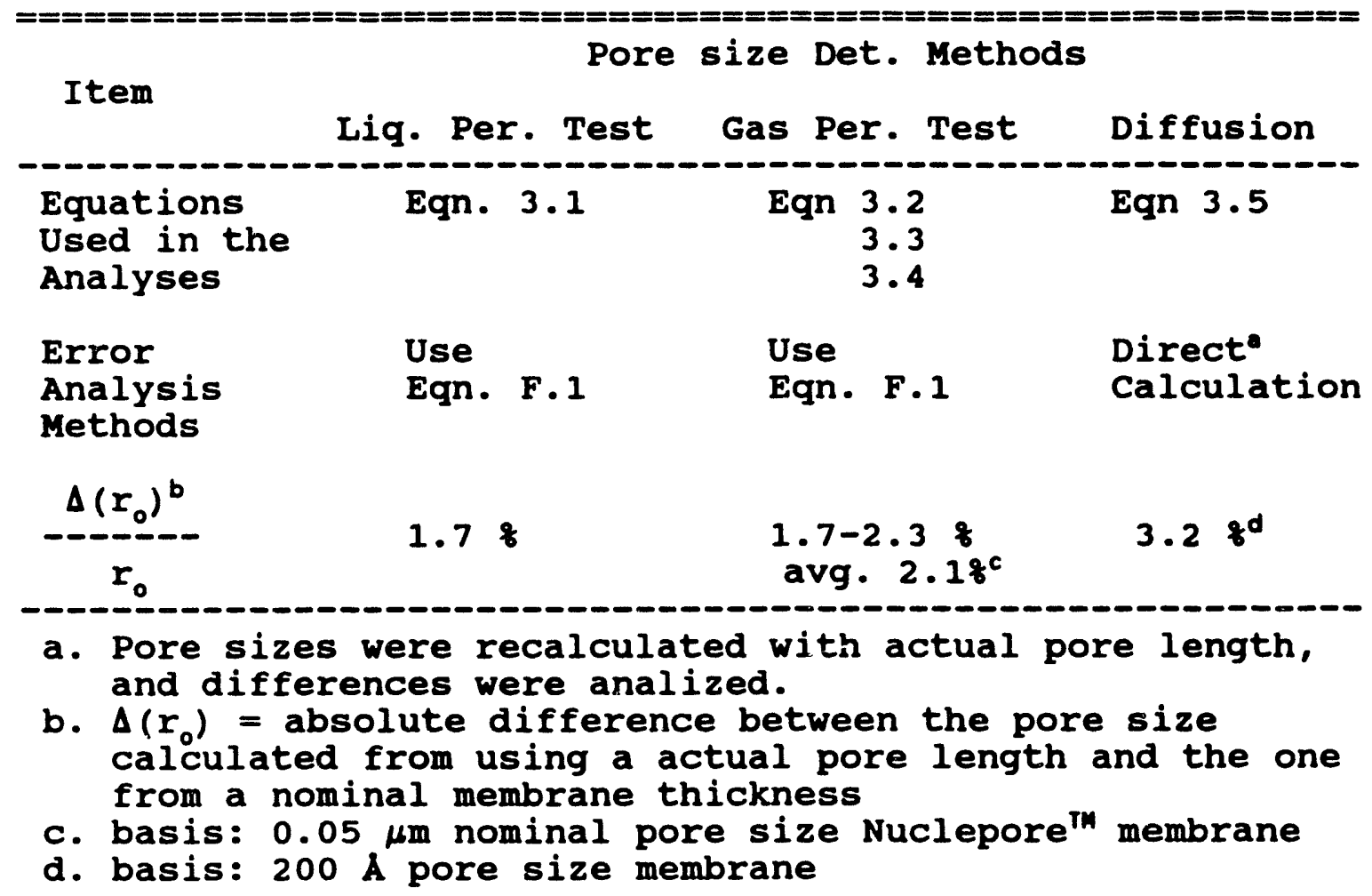


254

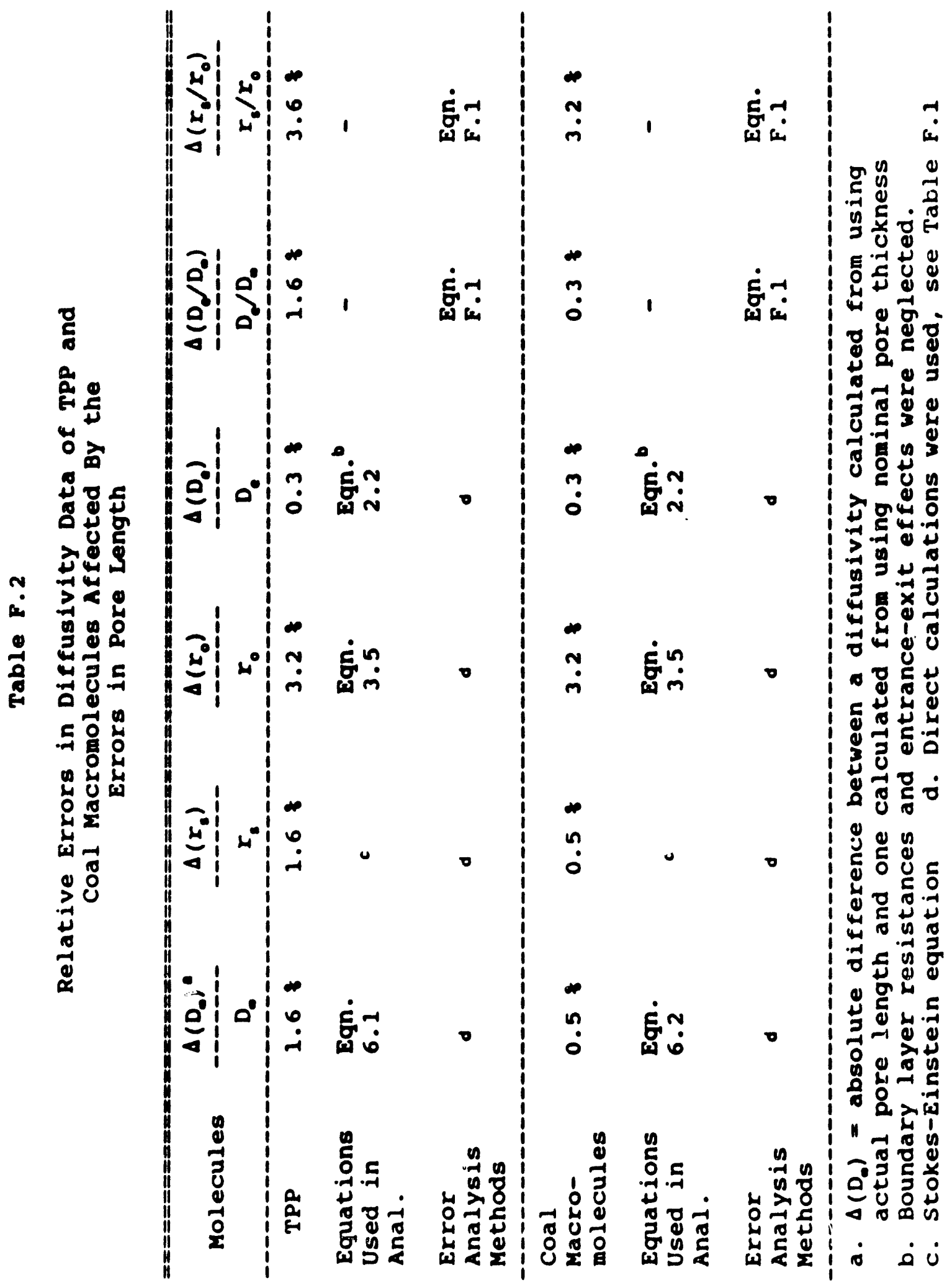


membrane product catalog ( \pm 15\%) was used as a calculation basis. In Tables F. 3 and F.C., the results of error analysis related to the errors in pore densities are summarized. As shown in these tables, $15 \%$ error in the pore density also does not significantly affect the relative errors in diffusivity data or those in measured pore radii.

since the errors in pore densities of membranes were random type errors, the results presented in Tables F. 3 and F.4 did not give a clear explanation about how the experimental data points presented in Figures 6.5 and 7.5 were affected by these relative errors. Thus, sensitivity analyses were conducted to examine the effect of errors in pore density on the movements of experimental data points in Figures 6.5 and 7.5 . In Figure F.1, results from the sensitivity analysis of TPP experimental data are presented. As shown in this figure, the changes in TPP experimental data points in this figure are not significantly affected by the changes in pore densities of membranes. Also these experimental data points move along the theoretical curve as the pore densities are changed. Accordingly, it was concluded that the differences between the experimental data and the theoretical curve observed in Figure 6.5 were not attributed to the errors caused by using the nominal values of pore densities in the analyses of experimental data. A similar conclusion can also be drawn from the sensitivity analysis of coal experimental data shown in Figure F.2. 
Table F.3

Effects of Errors in Pore Density on Uncertainties of Pore sizes Determined from the Various Methods

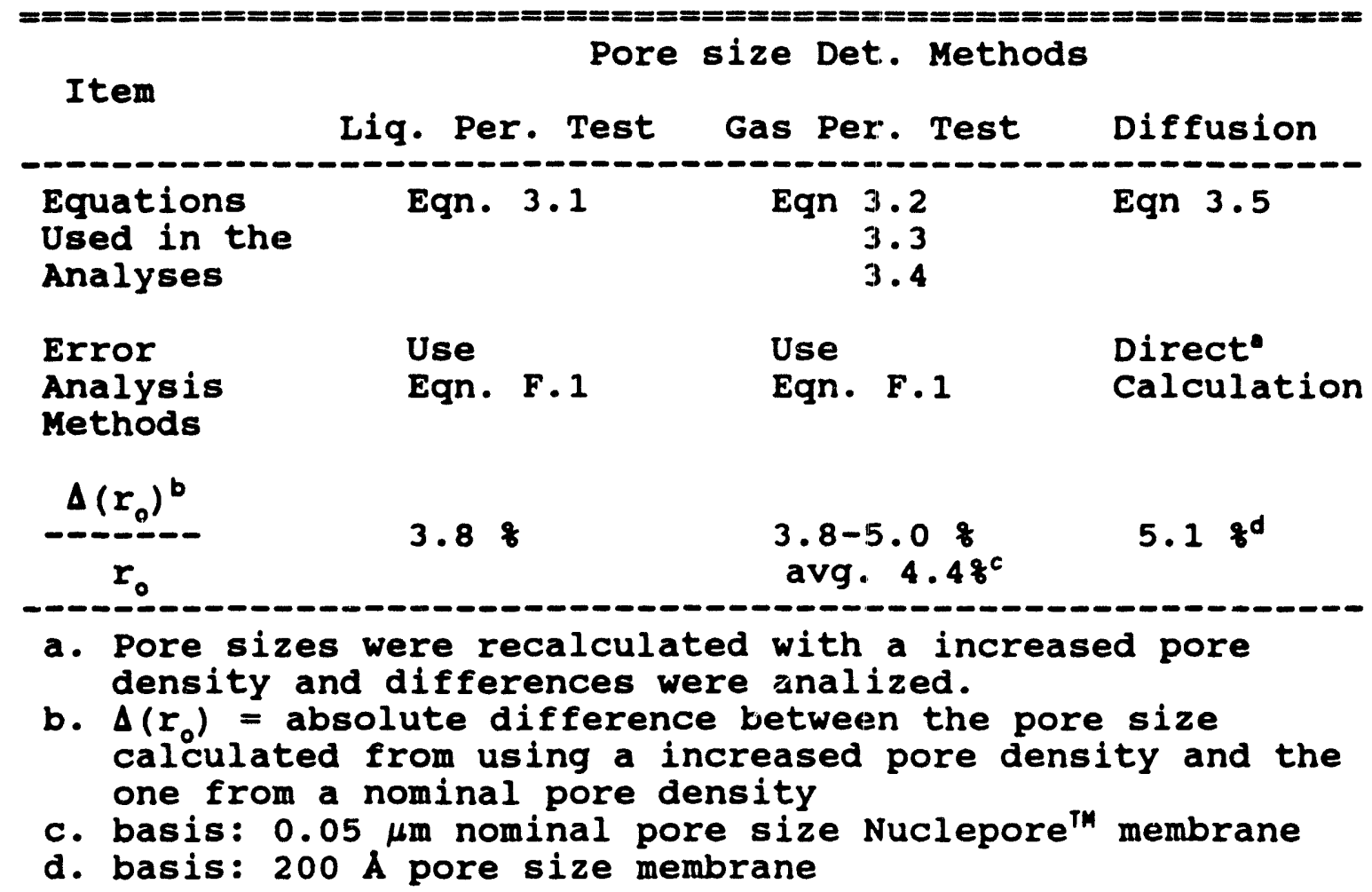




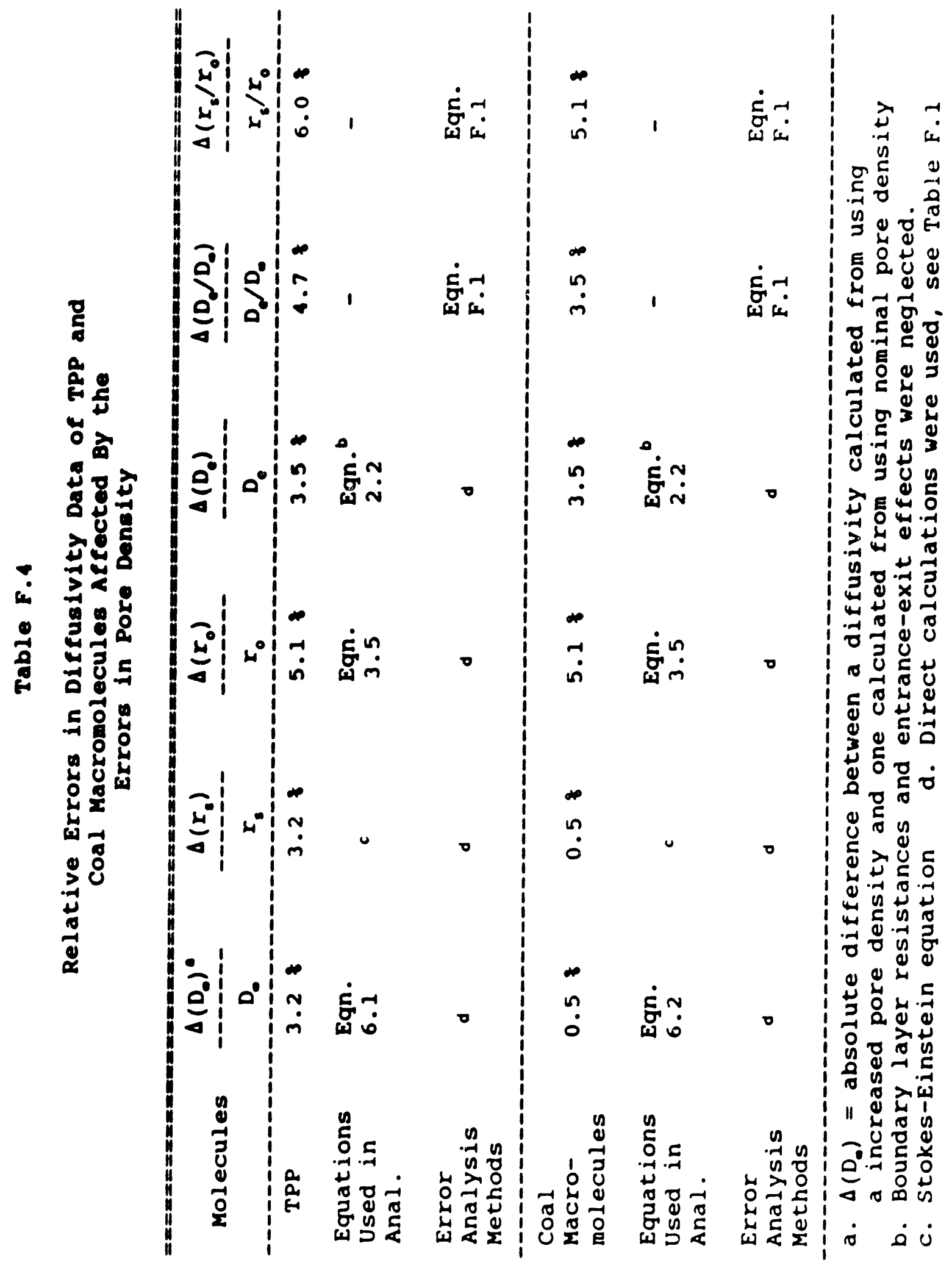




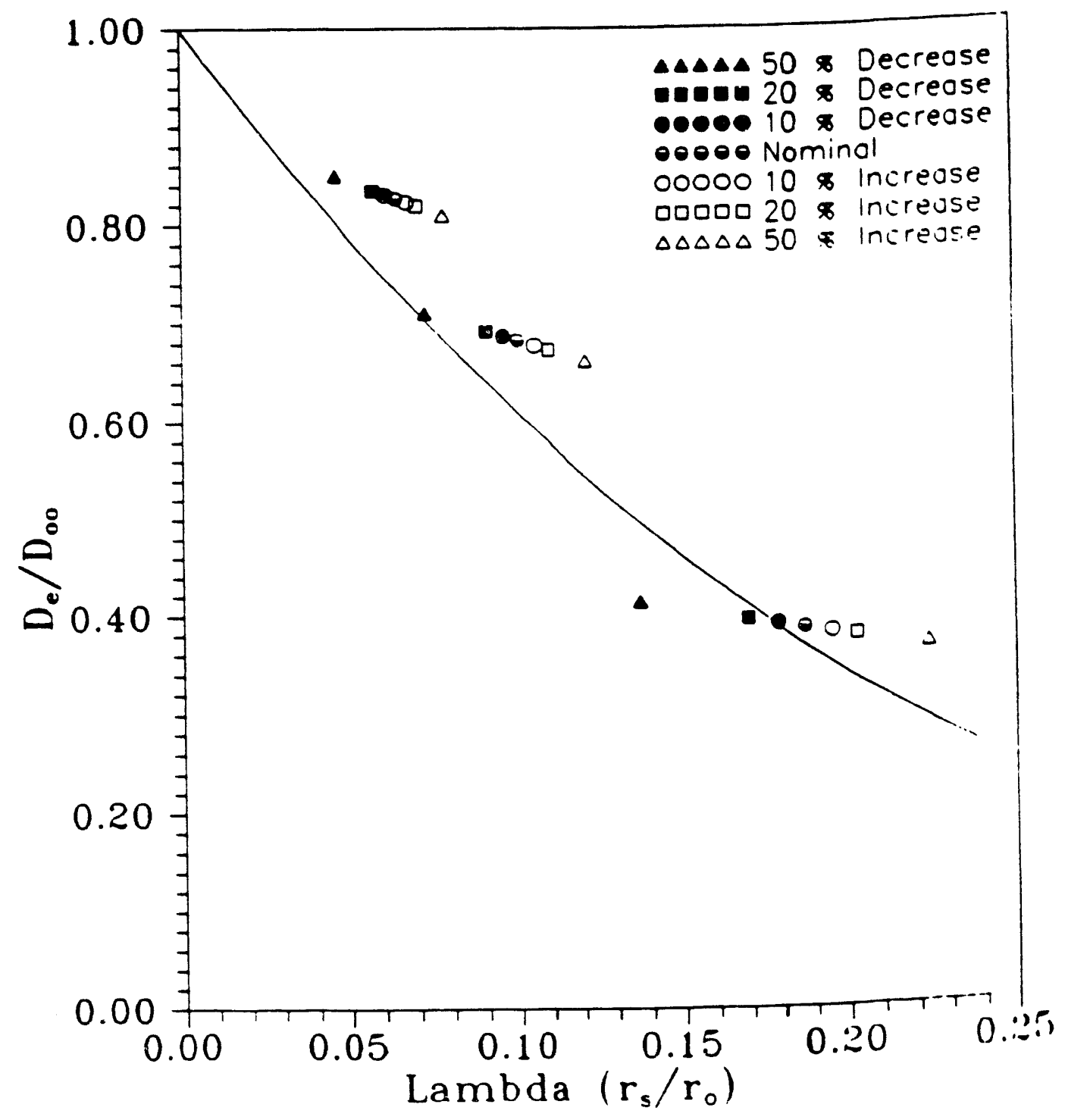

Figure E.I Sensitivity Analysis of TPP Diffusion lint 


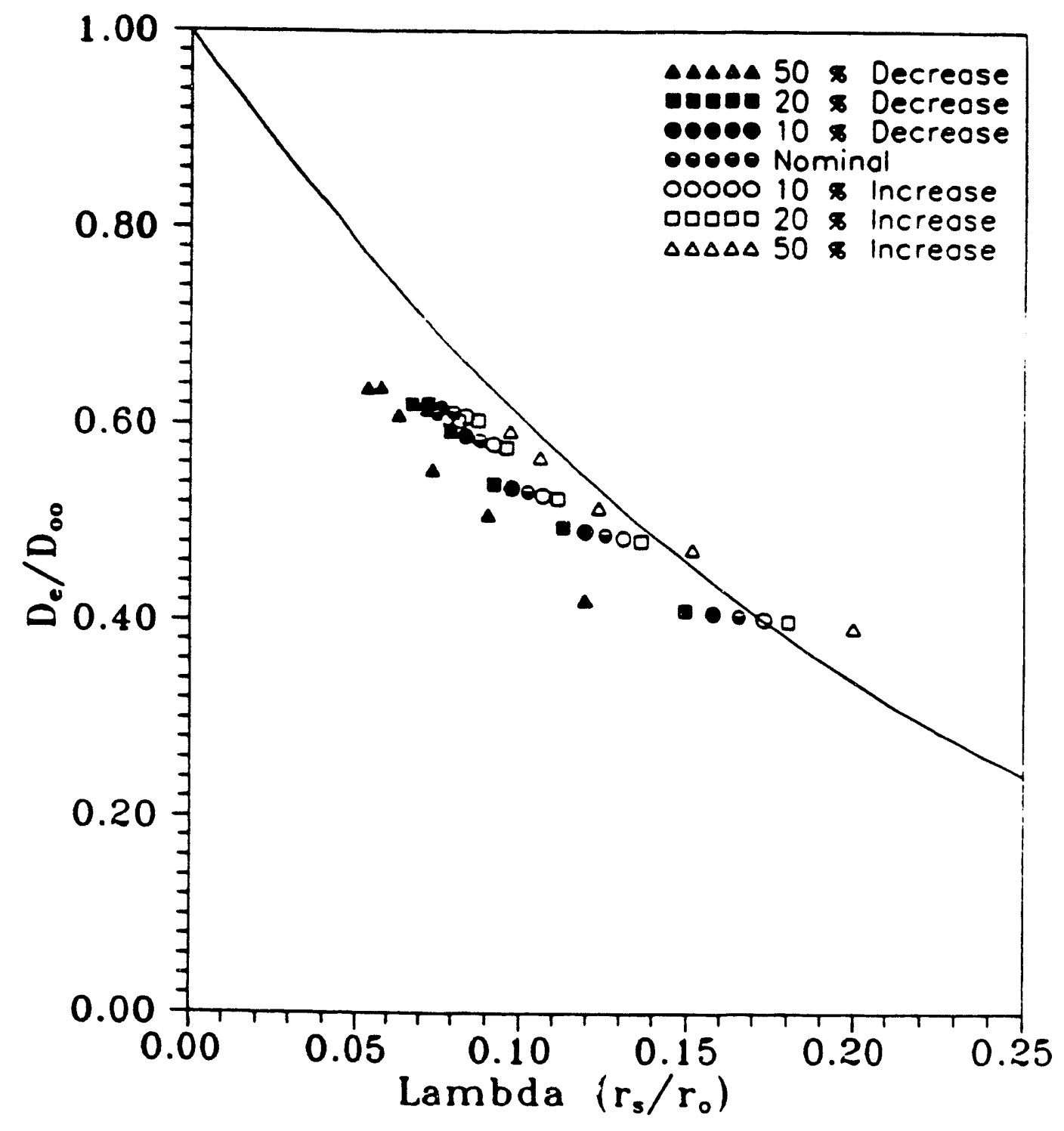

Figure F.2 Sensitivity Analysis of Coal Diffusion Data 


\section{Effects of Errors in Concentration Measurements}

The accuracy of diffusivity data or calculated pore sizes were not limited by the accuracies of concentration measurements where a gas chromatography or UV spectrometry were adopted as an analytical technique, since the measurement errors in these instruments are very low (usually less than \pm 1 of in the current research). However, an average relative measurement error in GPC analysis was found to be around \pm 2 in the current research. In the diffusion experiments of coal macromolecules, concentration measurements of samples taken from both compartments of the diffusion cell were conducted independently. Thus, a maximum uncertainty in measured diffusivity data of coal macromecules occurred when the errors in concentration measurements of samples had opposite signs.

The average error in the bulk diffusivity of coal fractions calculated on the basis of this maximum error in the measurements using GPC was found to be $8.3 \%$, while the average error in the effective diffusivity data was 7.68 . In these analyses, 12 experimental data of bulk diffusivity and six data of effective diffusivity were used. 
APPENDIX G

BASIC PROGRAMS FOR DATA ACQUISITION

AND HANDLING 


\section{BASIC Programs for Data Acquisition}

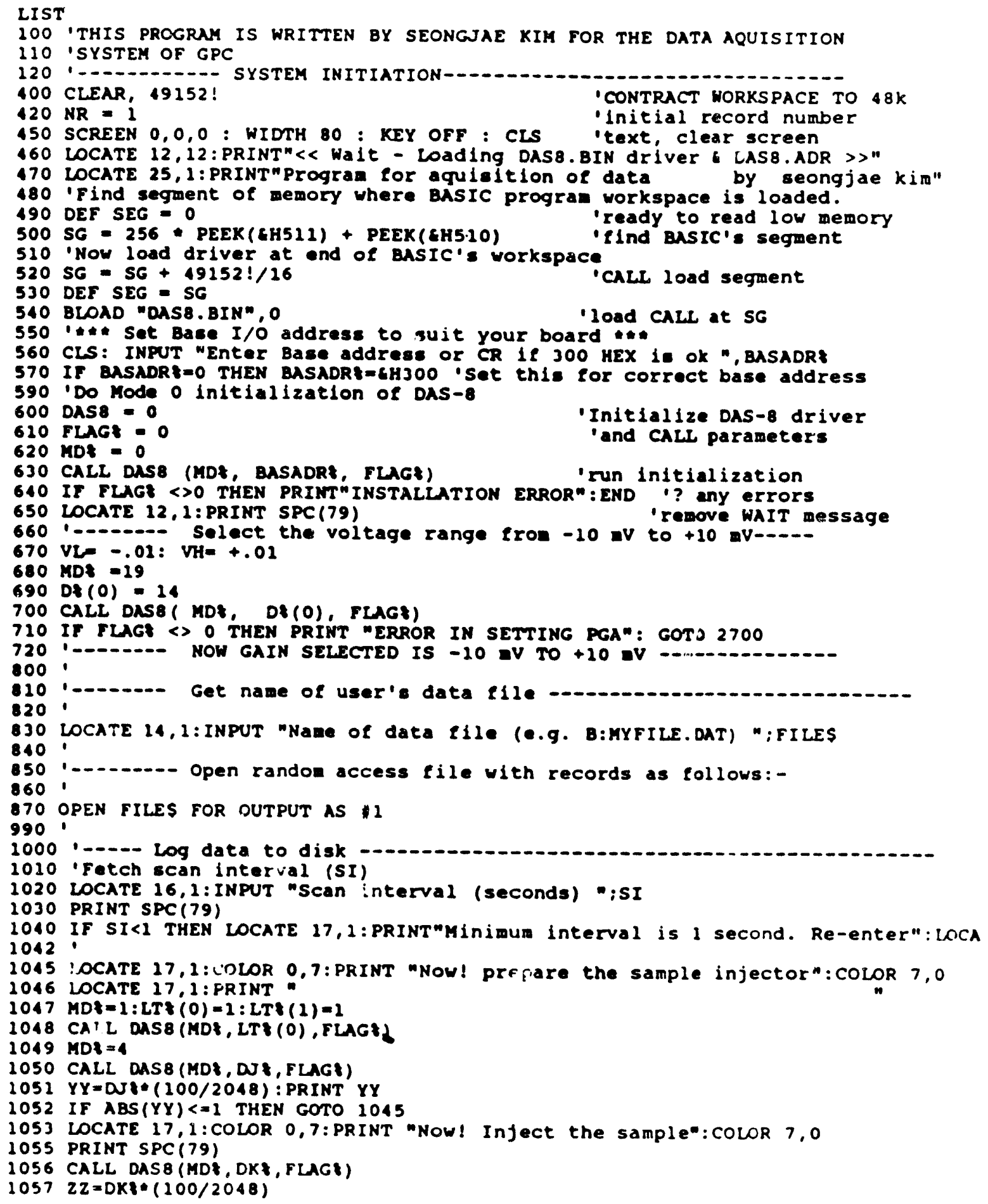




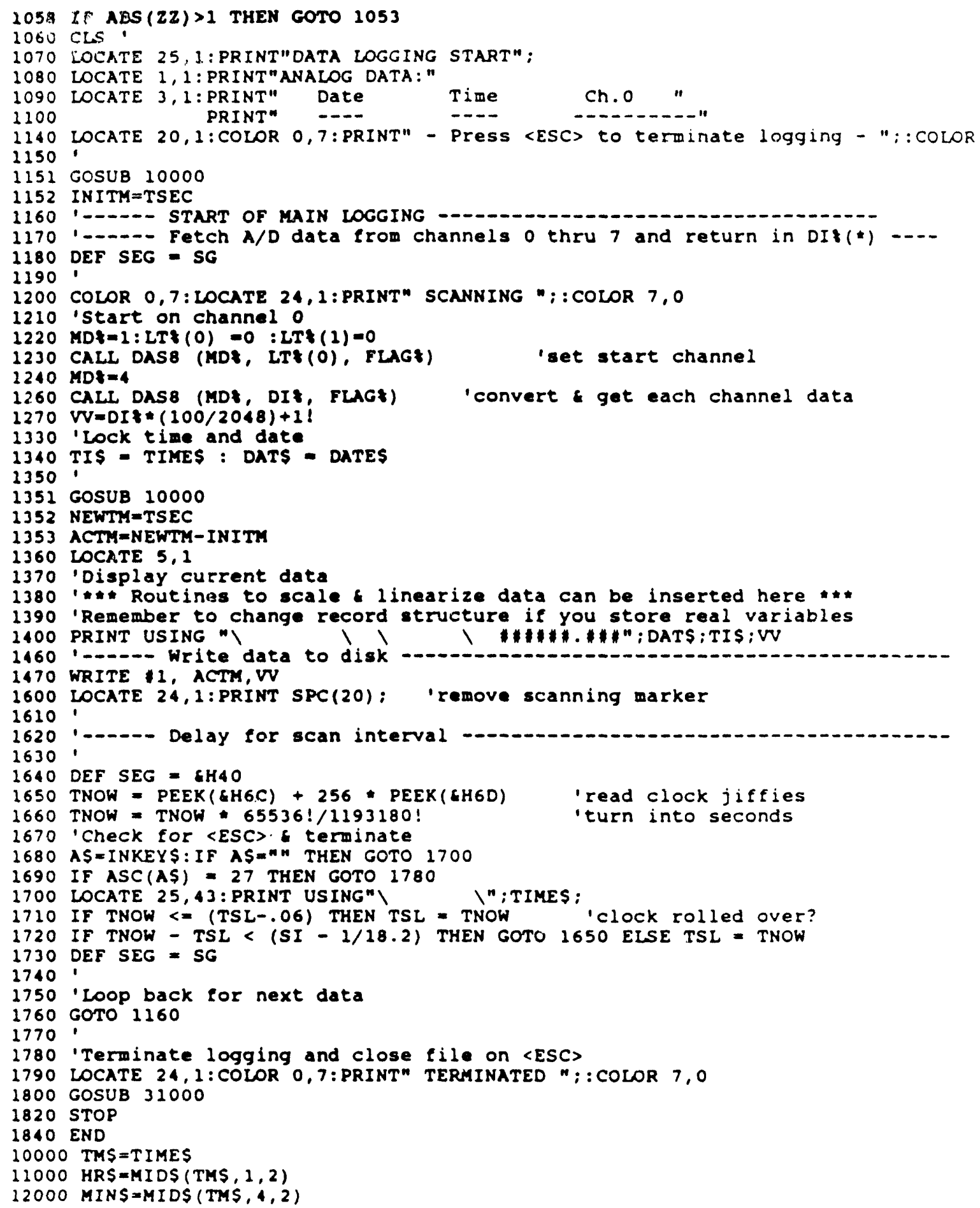




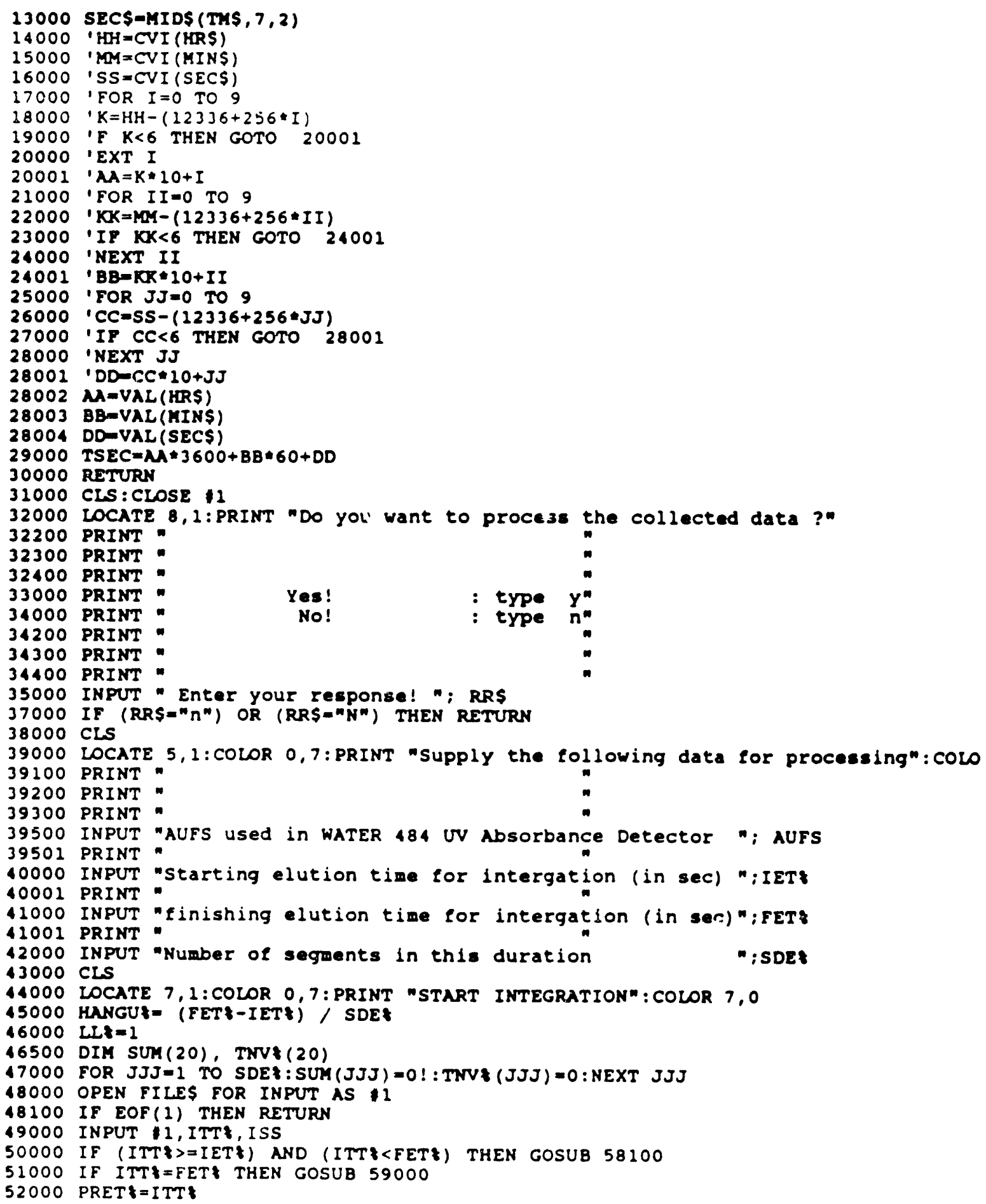




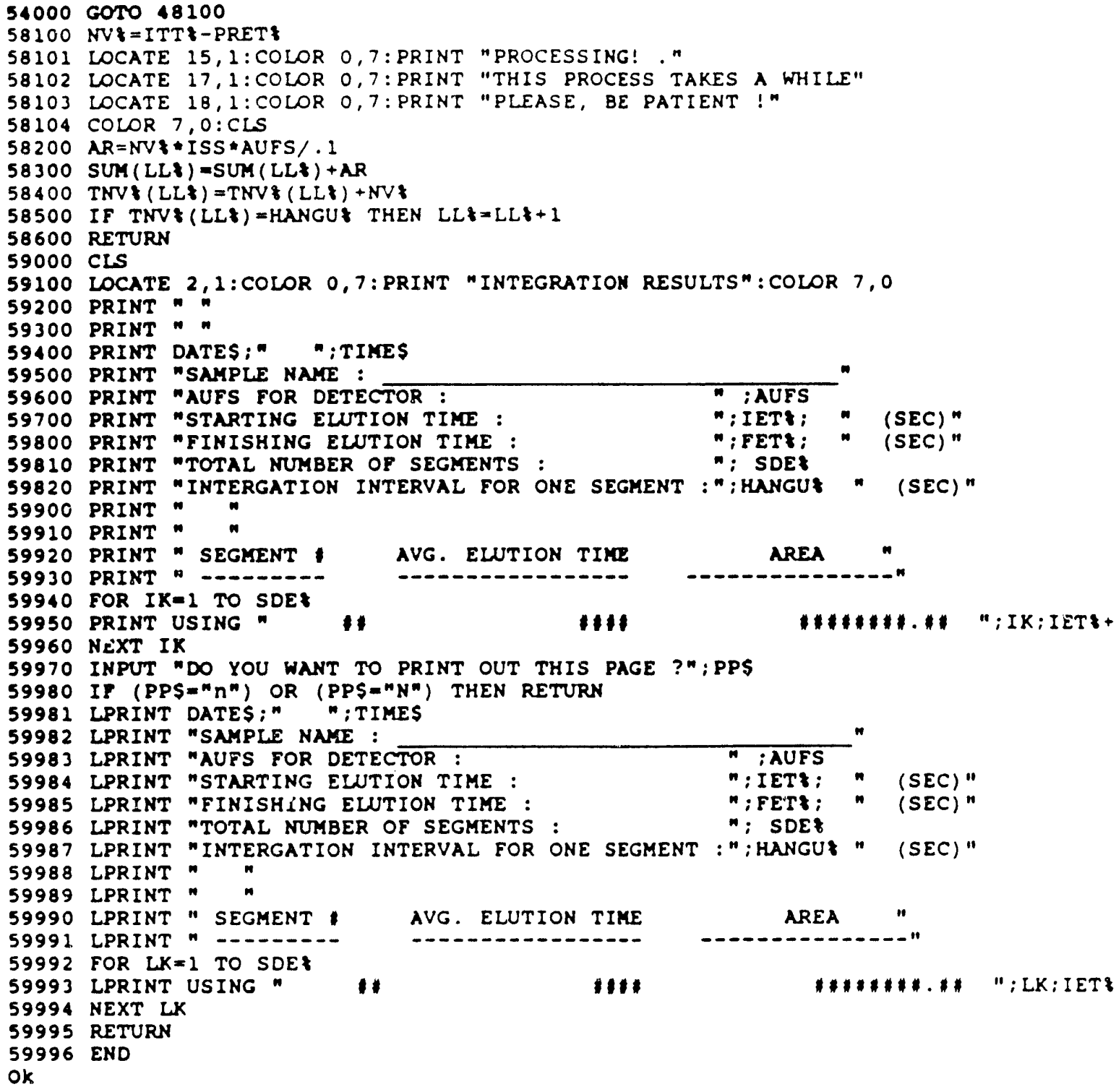




\section{APPENDIX H}

DIFFUSION IN ACTUAL ALUMINA POROUS MEDIA 
DIFFUSION IN ACTUAL ALUMINA POROUS MEDIA

Effective diffusivities were measured using tetraphenyl-porphine (TPP) in ethyl acetate. In our experiments, the diffusion apparatus shown in Figure H.I was used. The effective diffusivity values were obtained from a least square fit of the experimental data to an analytical solution curve using methods similar to Rhee (1).

\section{EXPERIMENTS}

Materials. Four different alumina based porous media were used in the diffusion experiments. These were F-00, F40, Harshaw AL-01-4, and Amocat 1-C. F-00 is a uni-modal catalyst and F-40 a bi-modal catalyst. Their properties are given by wang (126). The other two are commercial catalyst:s.

rihe following chemicals were used as received: tetraphenyl-porphine (TPP) (Aldrich), and ethyl acetate (EA) (Fisher, certified A.C.S.). A UV-Visible spectrophotometer (Varian Co., Model DMSlOOS) was used in concentration analyses of the diffusing compound (TPP).

Equipment. The diffusion apparatus applied in this work was developed by Tsai (11). This app ratus, as shown in Figure H.1, was comprised of a glass diffusion cell, a glass stem, a wire screen basket and a magnetic stirring plate with a stirring bar. The glass diffusion cell served as a bath for the diffusion experiment and had a constant volume of $180 \mathrm{ml}$. The glass stem was designed to hold a 
stainless steel wire screen basket containing about one gram of extrudate catalyst. To eliminate external mass transfer resistance, a magnetic stirring plate with a stirring bar was used.

Experimental procedures for the diffusion study were as follows. Approximately one gram of the catalyst pellets were dried in an oven at $80^{\circ} \mathrm{C}$ for one day to remove moisture in the pores of the pellets. Dried pellets were put in a basket and soaked in a concentrated solution of known TPP concentration for about 24 hours. When the pellets were equilibrated they were taken out of the solution and rinsed with pure ethyl acetate (EA) in order to remove TPP that might have remained on the outer surface of the pellets and basket. Washing was rapidly performed eight times with four beakers, each of which contained $100 \mathrm{ml}$ of EA. After washing, catalysts were put in a glass diffusion cell containing about $180 \mathrm{ml}$ of EA. Figure H.1 shows the diffusion cell used for the experiments. Five ml of solution was sampled seven times from the diffusion cell and used for analysis. After analysis the solution was returned to the diffusion cell to maintain constant volume. The equilibrium concentration was attained in 24 hours. The concentration of TPP was found using visible spectrometry at $414 \mathrm{~nm}$. The calibration curve is presented in Figure H.2. Data produced by the analysis was analyzed with BASIC and SAS programs to calculate the effective diffusivities. The 
effective diffusivity values in the TPP-EA diffusion system are listed in Table 1. These values were determined from plots such as shown in Figure H.3 by least squares curve fitting.

Results of adsorption experiments with F-00, F-40, and Amocat 1-C indicated no preferential adsorption of TPP on the surface of the alumina pellets with ethyl acetate solvent.

\section{TEMPERATURE EFFECTS}

The effect of temperature on the diffusivity value of TPP was studied as well. Diffusivity values in AL-0104 catalysts at $0^{\circ} \mathrm{C}$ (last five values in the Table $\mathrm{H} .1$ ) did not reveal any significant differences from the values at room temperature.

Figure H.4 shows the adsorption of TPP (uptake curves) from a mineral oil solution using AL-0104 alumina catalyst from Harshaw Co. at two different temperatures. The rates of uptake are controlled by diffusion into the catalyst pores: however, the effects of elevated temperature on the rate of uptake do not appear to be significant, i.e. within the accuracy of the data the $25^{\circ}$ and $50^{\circ} \mathrm{C}$ data do not appear significantly different.

Figure H.5 shows similar uptake curves for coal derived macromolecules from THF solution in $0.6 \mathrm{~cm}$ spherical alumina catalysts A-202-HF from Kaiser Co. at $25^{\circ} \mathrm{C}$. The uptake rates appear to be diffusion controlled for the larger 
270

molecules (shorter elution times). Figure H.6 shows the effect of operating at the elevated temperature of $50^{\circ} \mathrm{C}$. Again, as with the TPP case in Figure H.4, the effect of temperature on the adsorption-diffusion phenomena does not appear to be pronounced. 
271

Table H. 1

Diffusivity Values in TPP-Ethyl Acetate system

\begin{tabular}{|c|c|c|c|c|}
\hline Catalyst & FOO & F 40 & AL-0104 & AMOCAT 1-C \\
\hline Diffusivity & $\begin{array}{c}\left(10^{-6} \mathrm{~cm}^{2} / \mathrm{s}\right) \\
6.17 \\
8.56\end{array}$ & $\begin{array}{c}\left(10^{-6} \mathrm{~cm}^{2} / \mathrm{s}\right) \\
1.46 \\
1.62\end{array}$ & $\begin{array}{c}\left(10^{-6} \mathrm{~cm}^{2} / \mathrm{s}\right) \\
2.34 \\
2.44 \\
2.76 \\
3.05 \\
2.88 \\
3.05^{\circ} \\
2.18^{\circ} \\
1.91^{\circ} \\
2.32^{\circ} \\
4.33^{\circ}\end{array}$ & $\begin{array}{c}\left(10^{-6} \mathrm{~cm}^{2} / \mathrm{s}\right) \\
3.03 \\
2.86\end{array}$ \\
\hline
\end{tabular}

a. at $0^{\circ} \mathrm{C}$ 


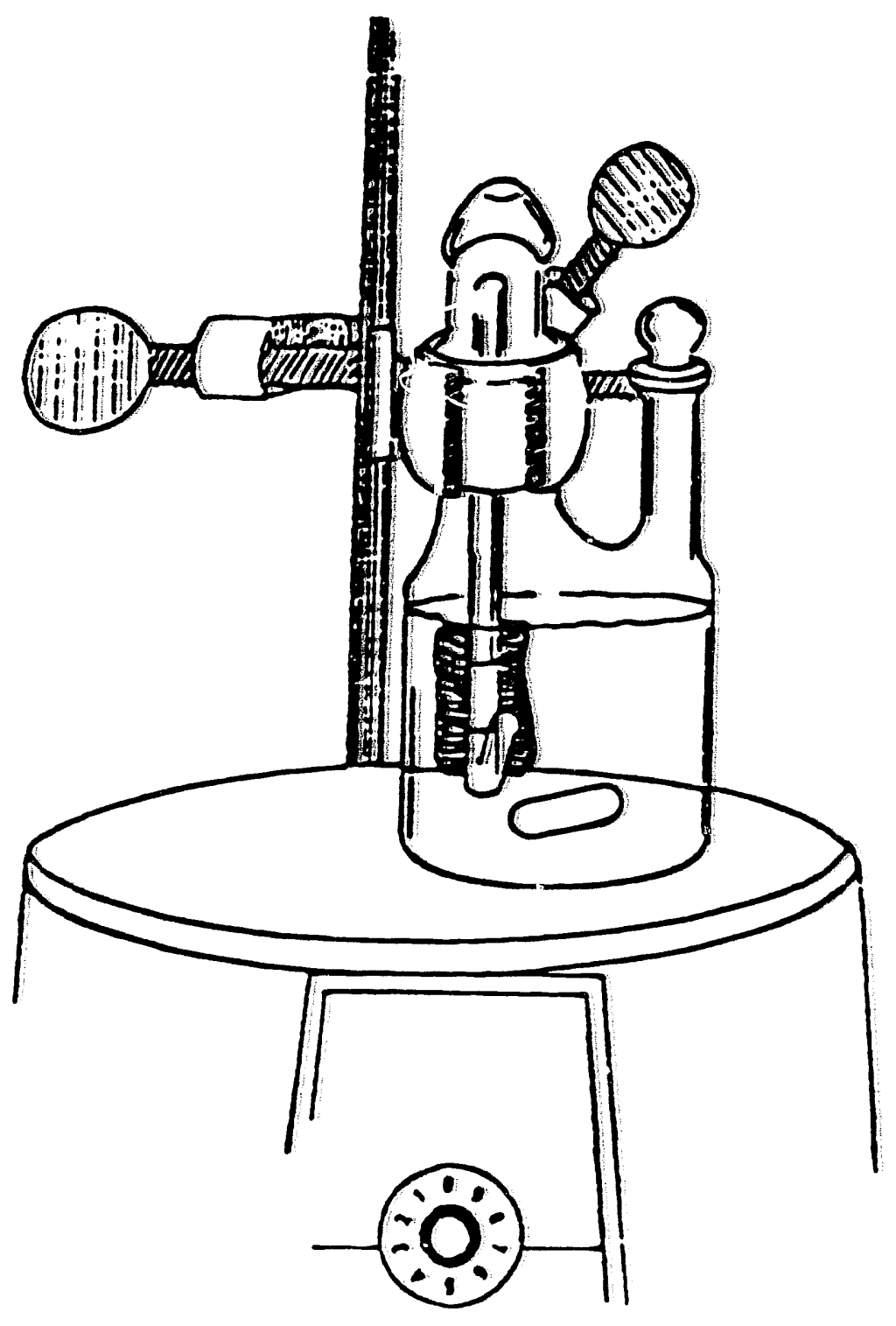

Figure H.I Diagram of glass diffusion cell 
UV CALIBRATION CURVE fOr TPP SOIUTION (UV Wovelength : $414.0 \mathrm{~nm}$ )

SLOPE $=0.74055$

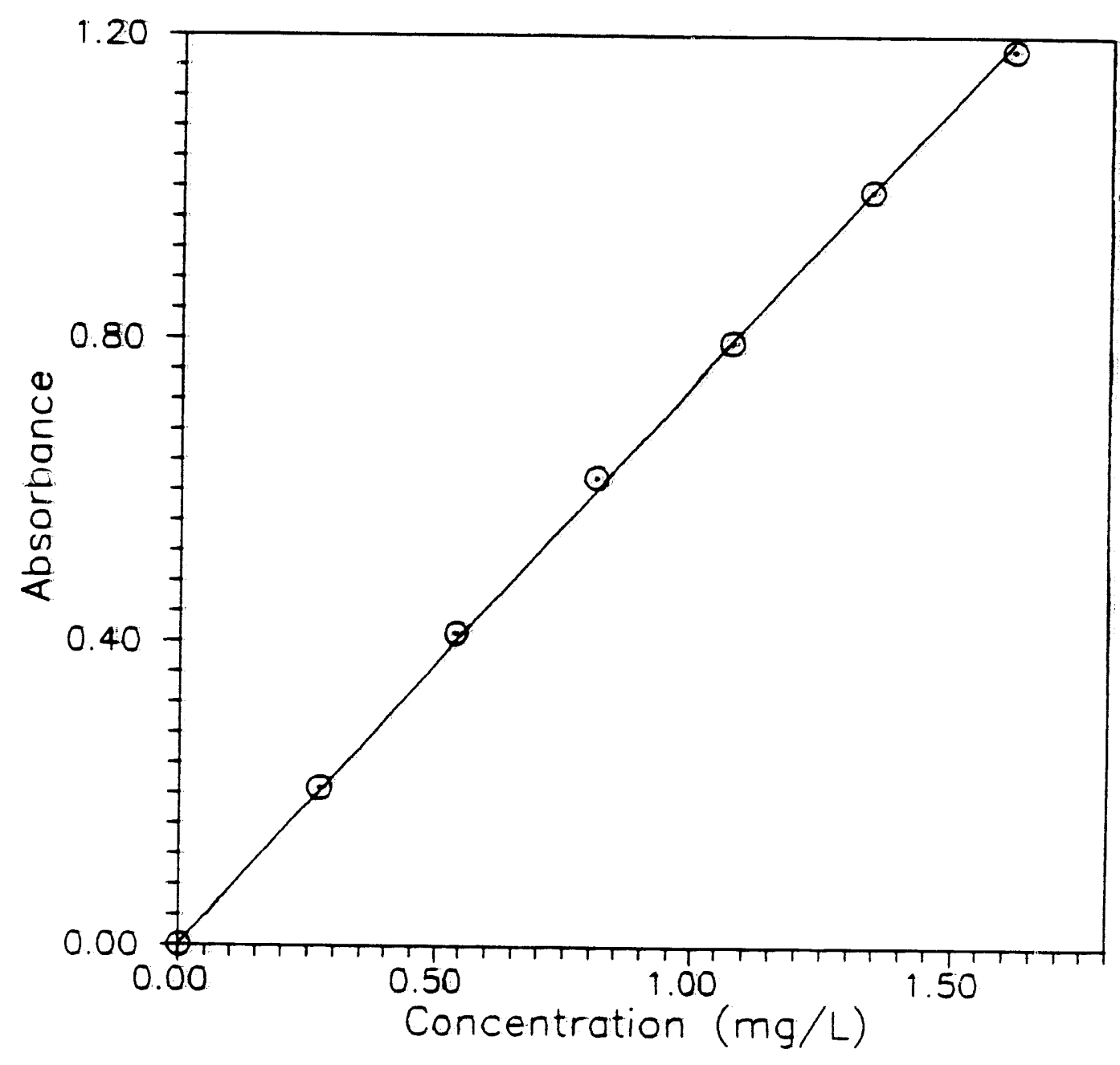

$\begin{aligned} \text { Figure H.2 Beer's Law plot for analysis of TPP } & \\ & \text { in ethyl aretate at }\end{aligned}$ 


$$
\begin{aligned}
& \text { (TPP in Ethyl Acetate) } \\
& D e=1.62 \times 10^{-6} \mathrm{~cm}^{2} / \mathrm{s}
\end{aligned}
$$

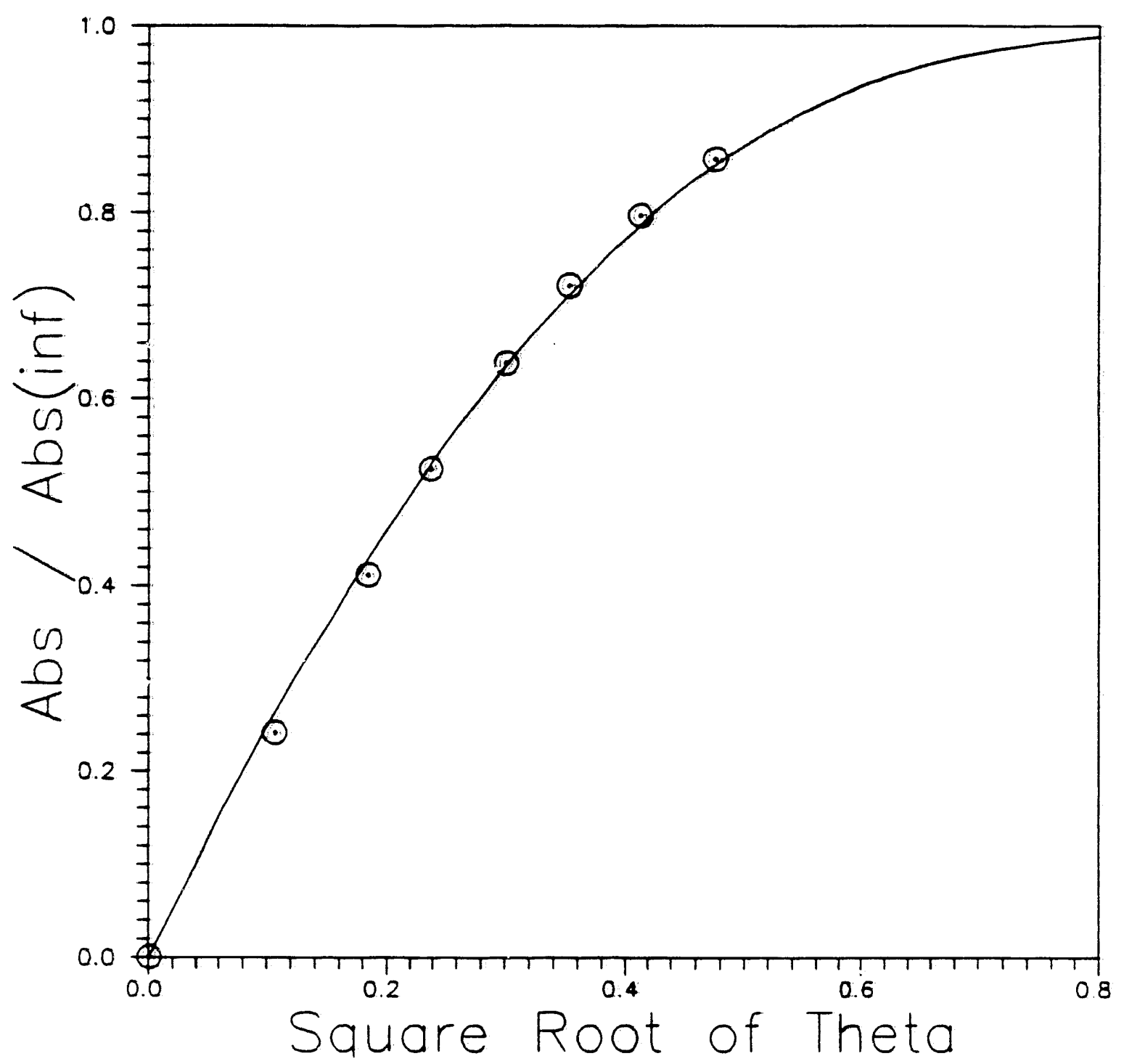

Figure H.3 Typical diffusivity data for TPP in ethyl acetate systen in catalyst $F-40$ 
Adsorption Experiment with TPP and Mineral Oil

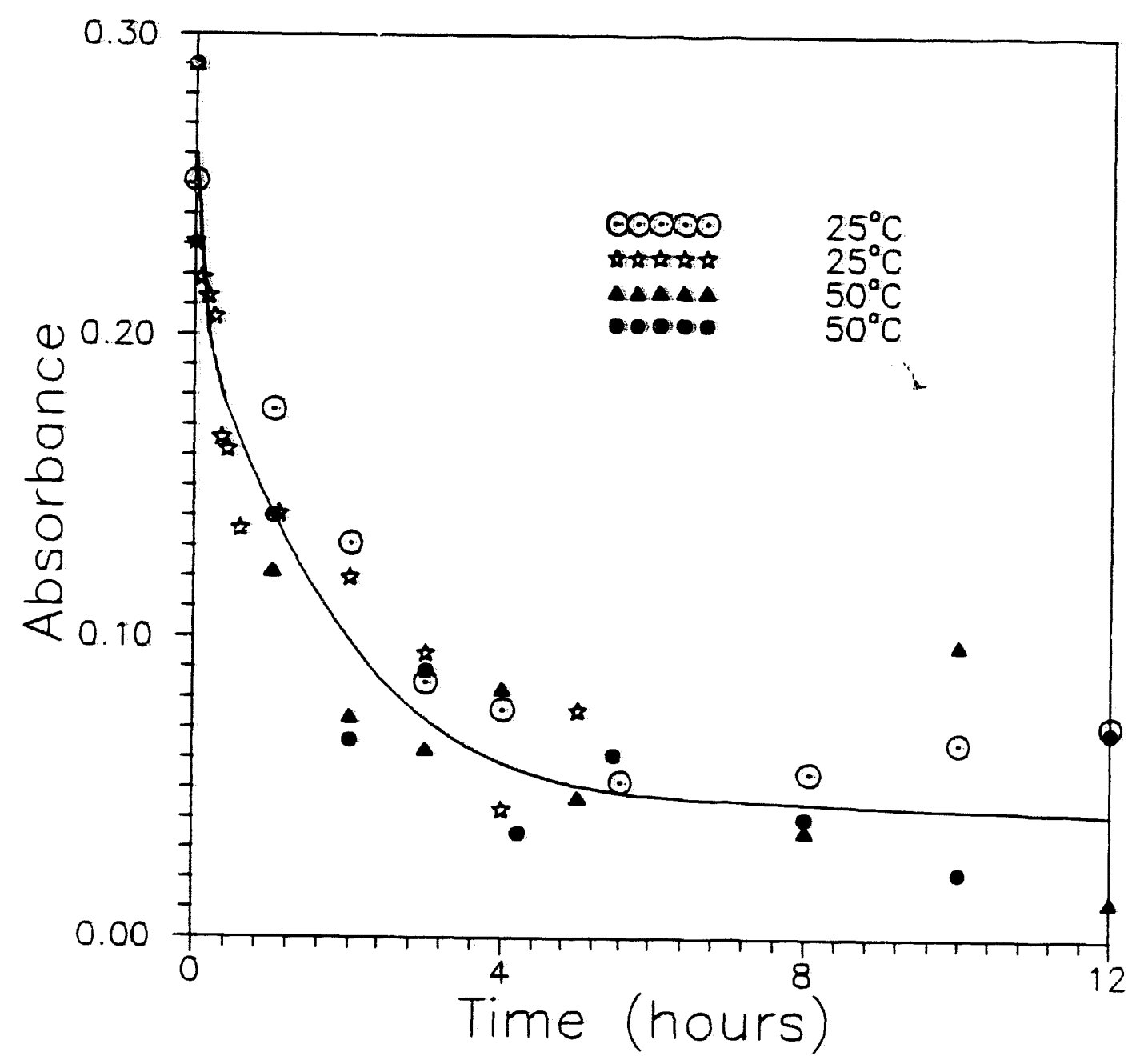

Figure H.4 Diffusional adsorption uptake curves for TPP/nineral oil solution on alumina pellets 


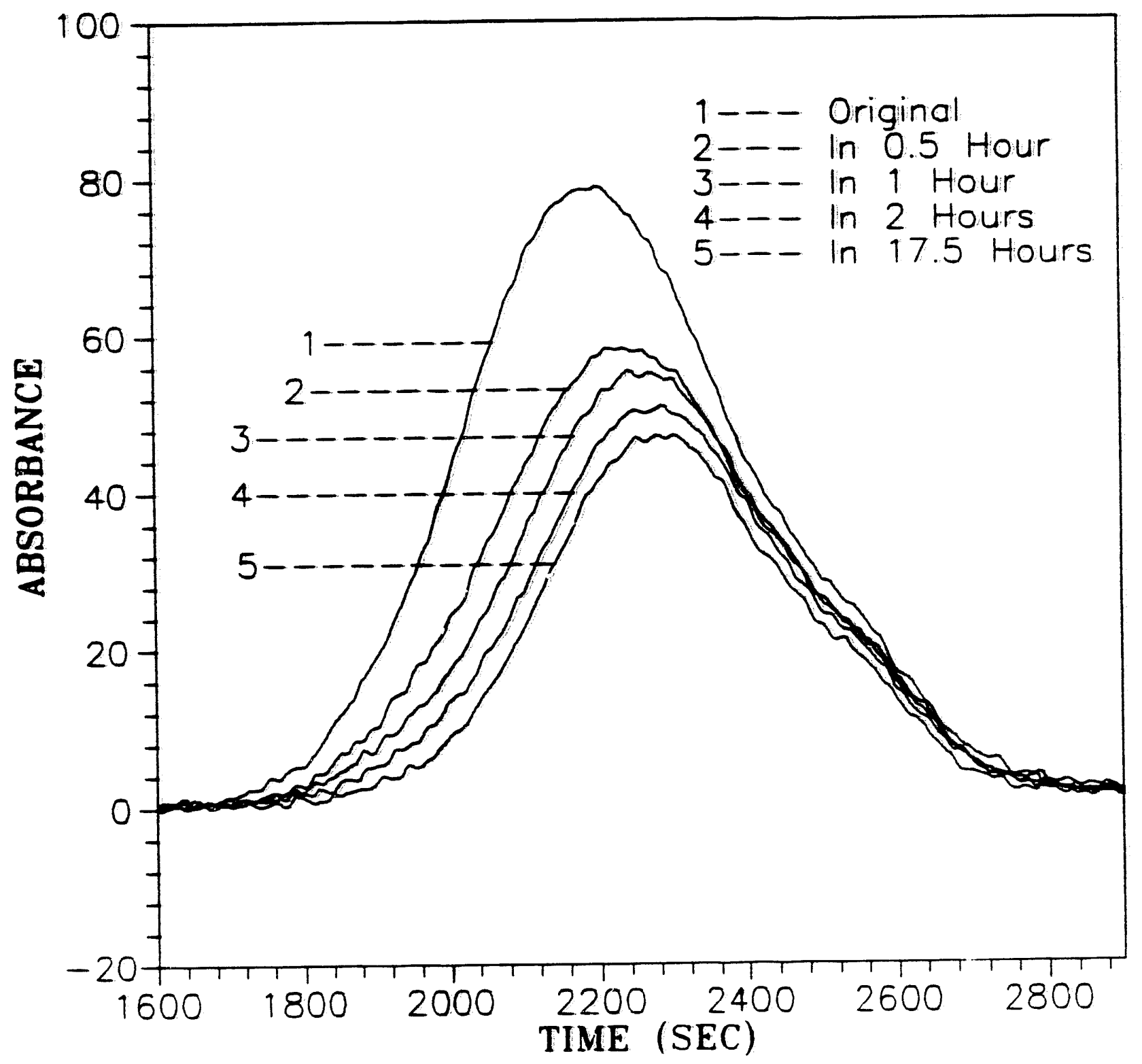

Pigure H.5 GPC chromtograns of diffusional uptake curves of coal nacromolecules from THF solution at $25^{\circ} \mathrm{C}$ on alumina pellets 


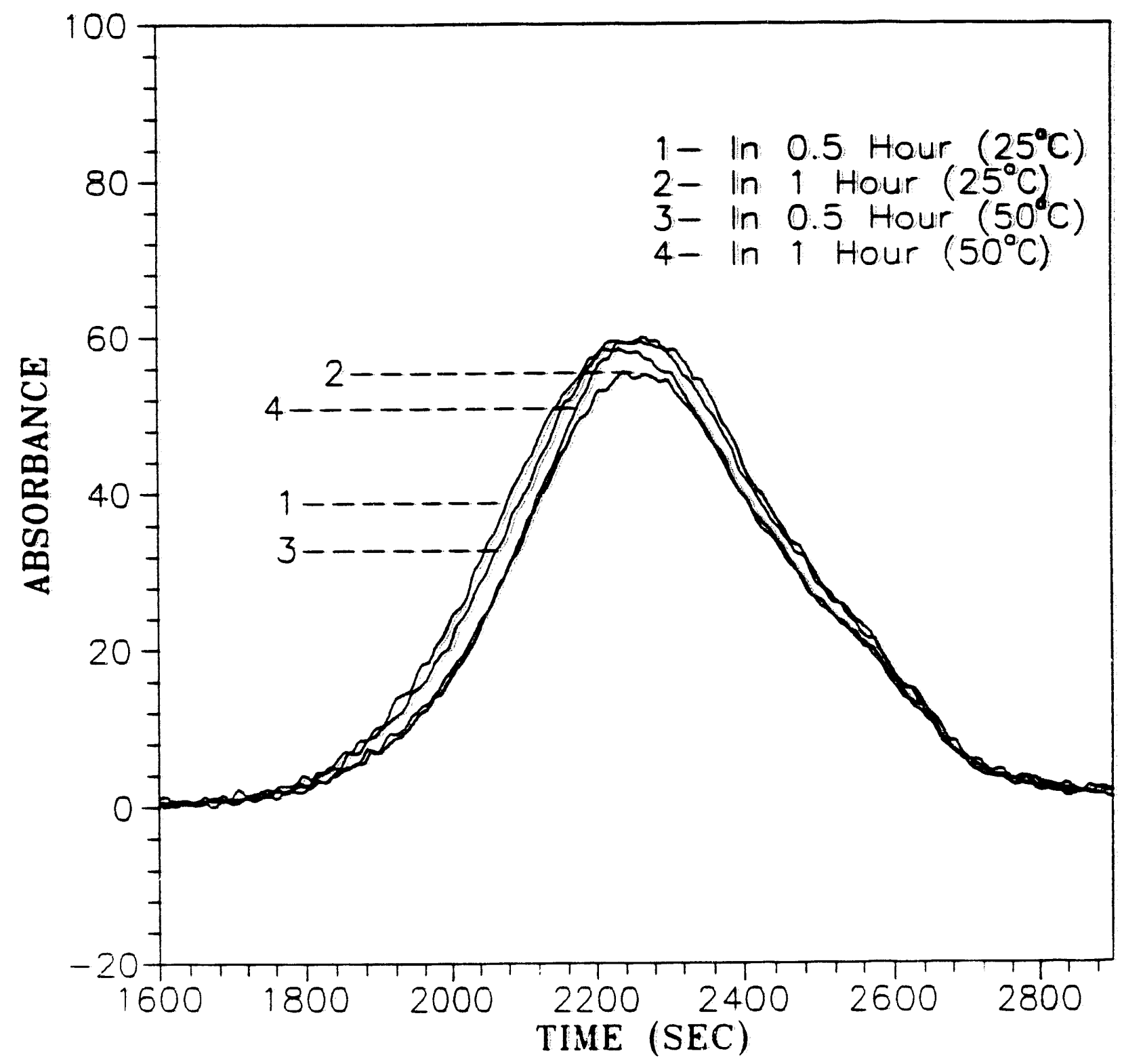

Figure H.6 GPC chromatograms of diffusional uptake curves of coal macromolecules from THF golutions at $25^{\circ} \mathrm{C}$ and $50^{\circ} \mathrm{C}$ on alumina pellets 


\section{APPENDIX I MOMENCIATURE}




\section{Nomenclature}

a : radius of spherical pasticle confined in a liquid filled pore

$a_{i}:$ polynomial constants

A : area of membrane for diffusion or permeability test

A : unit area of pore

b : average distance between the axes of adjacent pores

c. : concentration of solute at the high concentration side of diffusion cell compartment

$c_{1}, c_{2}$ : average concentration of solute adjacent to pores

<c> : local cross-section average concentration in Eqn. 2. 11

$\left\langle c_{0}\right\rangle,\left\langle c_{l}\right\rangle$ : cross-section average concentration of solute at the ends of pore

$c_{b 1}, c_{b 2}$ : average bulk concentration of solute adjacent to diffusional boundary layers

c : concentration of solute at the low concentration side of diffusion cell compartment

$c_{H}{ }^{\circ} \quad$ : initial concentration of solute at high concentration side of diffusion cell compartmert

$c_{L}^{\circ} \quad$ : initial concentration of solute at low concentration side of diffusion cell compartment

c : local molar concentration of solute in pore

<c> : molar concentration of solute averaged over pore cross section

c. : solute concentration in bulk solution

$c_{0}, c_{1}$ : solute concentration in external solutions adjacent to membrane surfaces

d : diameter of membrane area for diffusion 
D. : bulk diffusivity (diffusion coefficient)

D. : effective diffusivity (diffusion coefficient)

D, : surface diffusivity (diffusion copficient)

E : interaction energy between solute and pore wall

$E_{b}$ : interaction energy potential in the bulk solution

$E_{p} \quad$ : interaction energy potential in the pore

F : enhanced drag coefficient, Eq. 2.6

$f(\lambda)$ : hindrance correction factor

G : $\operatorname{lag}$ coefficient, Eq. 2.6

9 : function describing axial concentration variations in pore, Eq. 2.10

$q_{c}$ : gravitational constant

H : applied pressure head of water in Eq. 3.4

$K_{2}$ : mass transfer coefficient in Eqn A.1

$x_{r}$ : integral of inverse enhanced drag coefficient, Eq. 2.13

k : Boltzmann constant

$K_{p}$ : partition coefficient $<c>/ c_{\text {. at equilibrium }}$

1 : pore length

$M$ : molecular weight of solute

$N$ : local molar flux

$<N>$ : molecular flux averaged ever pore cross section

$N_{A}$ : molar flux of solute A (moles $/ \mathrm{cm}^{2}$ )

$n$ : number of pores per unit area of membrane surface (pores $/ \mathrm{cm}^{2}$ )

$N_{\text {Re }}$ : Reynolds number $\left(\omega d^{2} p / i\right)$

$\mathrm{N}_{s c}$ : Schmidt number $\left(\mu / \rho D_{-}\right)$ 
$\begin{aligned} N_{s h} & : \text { sherwood number }\left(d / D_{-} R_{b}\right) \\ p_{1}, p_{2} & : \text { pressures of iniet gas flow }\left(p_{1}\right) \text { and outlet gas }\end{aligned}$ flow $\left(p_{2}\right)$ in $E q \cdot 3.4$

$\Delta \mathrm{p} \quad$ : hydraulic pressure difference across membrane, "upstream" minus "downstream"

Q : volumetric flow rate of liquid in Eq. 3.1

Q : total molar flux of gas in Eq. 3.2

$Q_{k}$ : Knudisen molar flux of pore in Eq. 3.2

Q. : viscous molar flux of gas in Eq. 3.2

$R_{b}$ : boundary layer resistance

$R_{w} \quad$ : membrane resistance

$R_{T}$ : total transfer resistance ( $\mathrm{sec} / \mathrm{cm}$ )

R. : gas constart $\left(8.314\right.$ N.m/g-mole $\left.{ }^{\circ} \mathrm{K}\right)$

5 : radial position in pore (dimensional)

$r_{\text {s }}$ : stokes-Einstein radius of solute

$r_{0}$ : average pore radius of membrane

$t$ : diffusion time (sec)

I : temperature $\left({ }^{\circ} \mathrm{K}\right)$

$U$ : solute velocity along pore axis

$\mathrm{V}$ : unperturbed solvent velocity

$V_{H} \quad$ : volume of high concentration side of diffusion cell compartment

$v_{L} \quad$ : volume of low concentration side of diffusion cell compartment

$v_{i}$ : elution volume of GPC

$x_{m}$ : mean external length of molecules as du. ined in Eqn 6.7

$z$ : axial position in pore (dimensional) 
a : constant term in empirical boundary layer resistance equation

$\delta \quad:$ boundary layer thickness

$\epsilon \quad$ : porosity

r: tortuosity

$\lambda$ : the ratio of molecular diameter to pore diameter

$\Delta$ : absolute differences for error analysis

B : dimensionless radial position, $\boldsymbol{r} / \boldsymbol{r}_{0}$

$\mu$ : viscosity of solvent

$\omega$ : stirring speed

$p$ : density of solvent

$\zeta$ : general coordinates describing molecular position

$v$ : general coordinates describing molecular orientation

$\xi$ : general coordinates describing nolecular conformation

$\theta$ : angle between the axis of the molecular rotation and the unit normal vector of molecules

- : ratio of minor to major axis 

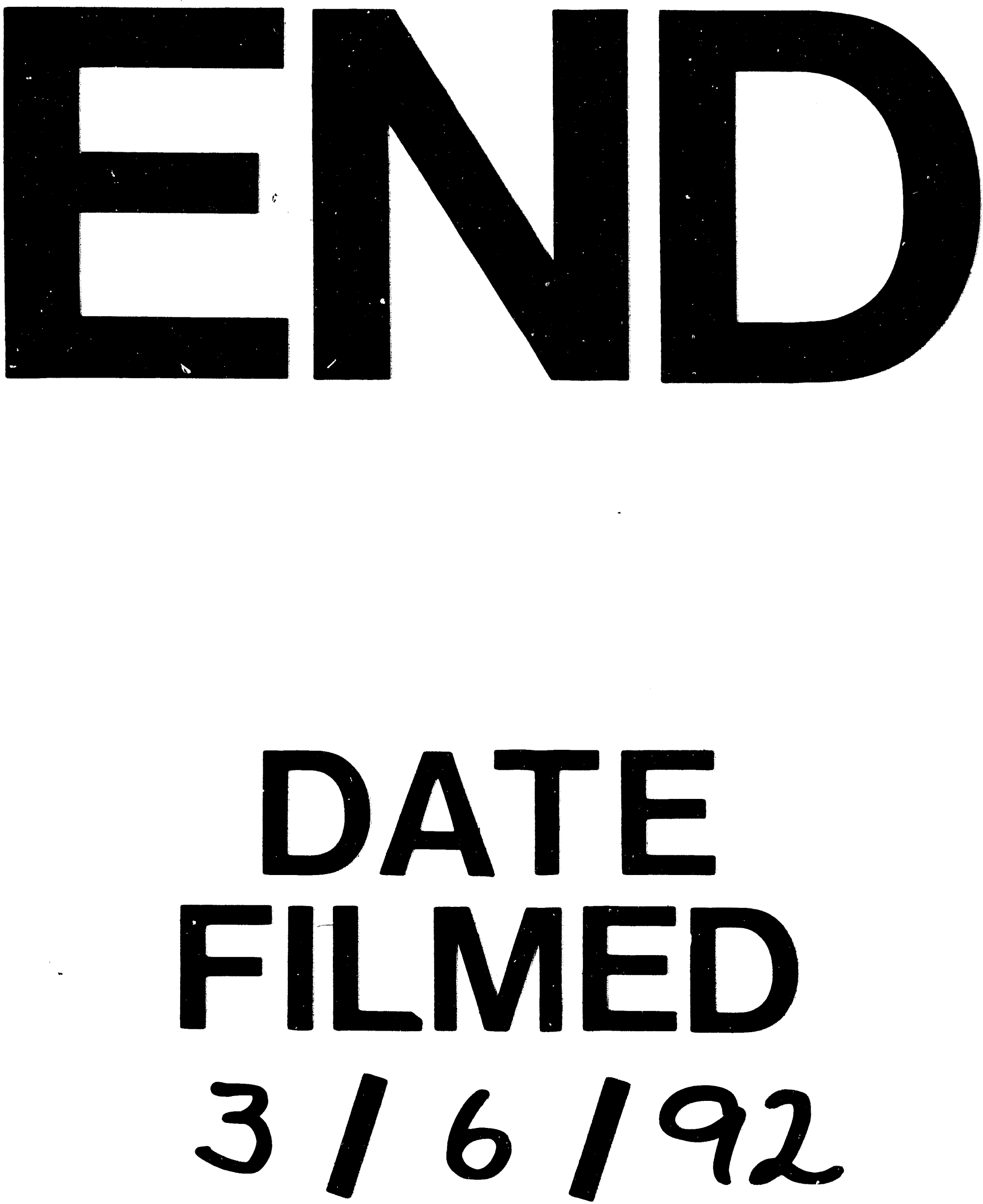